\title{
Christoph Höck
}

\section{Zur syntaktischen \\ und kommunikativen Struktur slavischer Partizipial- und Gerundiokonstruktionen}

Verlag Otto Sagner München · Berlin · Washington D.C.

Digitalisiert im Rahmen der Kooperation mit dem DFG-Projekt „Digi20“

der Bayerischen Staatsbibliothek, München. OCR-Bearbeitung und Erstellung des eBooks durch den Verlag Otto Sagner:

http://verlag.kubon-sagner.de

() bei Verlag Otto Sagner. Eine Verwertung oder Weitergabe der Texte und Abbildungen, insbesondere durch Vervielfältigung, ist ohne vorherige schriftliche Genehmigung des Verlages unzulässig. 


\title{
Slavistische BeIträge
}

\author{
BEGRÜNDET VON \\ ALOIS SCHMAUS \\ HERAUSGEGEBEN VON
}

JOHANNES HOLTHUSEN · HEINRICH KUNSTMANN · JOSEF SCHRENK

REDAKTION

PETER REHDER

Band 132

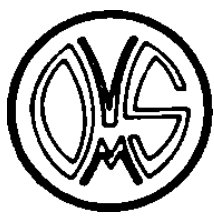

VERLAG OTTO SAGNER

MÜNCHEN 


\title{
CHRISTOPH HÖCK
}

\author{
ZUR SYNTAKTISCHEN \\ UND KOMMUNIKATIVEN STRUKTUR \\ SLAVISCHER PARTIZIPIAL- \\ UND GERUNDIALKONSTRUKTIONEN
}

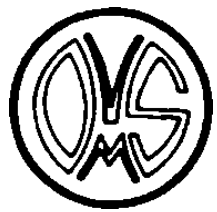

VERLAG OTTO SAGNER - MÜNCHEN

1979 


\section{Meinem Vater Joseph Höck zum Gedenken}

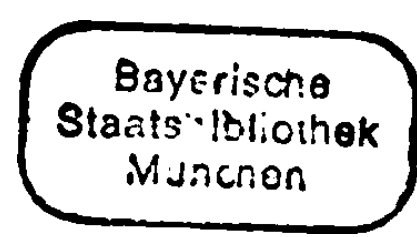

ISBN 3-87690-165-0

Copyright by Verlag Otto Sagner, München 1979 Abteilung der Firma Kubon \& Sagner, München Druck: Alexander Grossmann

Fäustlestr. 1, D-8000 München 2 
Vorliegende Untersuchung wurde im Wintersemester 1978/79 vom Fachbereich 12 "Altertumskunde und Kulturwissenschaften" der Ludwig-Maximilians-Universität München unter dem Titel:

"Zur funktionalen Spezifizierung russischer Partizipial- und Gerundialstrukturen (mit vergleichenden Hinweisen auf andere Slavinen)"

als Dissertation angenommen.

Studium und Promotion wurden mir ermöglicht durch stipendien nach dem Bayer. Begabtenförderungsgesetz und der studienstiftung des deutschen Volkes. Dafür sei hier gedankt.

Herrn Prof. Dr. Baldur Panzer, der die Arbeit betreut hat, bin ich für vielseitige Unterstützung $z u$ hesonderem Dank verpflichtet. Ebenso danke ich Herrn Prof. Dr. Josef Schrenk für seine Hilfsbereitschaft.

München, im März 1979

Christoph Höck 
A B K U R Z U N G E N

Die im Text verwendeten Abkürzungen für Quellenangaben sind im allgemeinen so gewählt, daß sich ihre Bedeutung aus dem Literaturverzeichnis ohne weiteres ergibt.

\begin{tabular}{|c|c|c|}
\hline \multirow[t]{7}{*}{ Vgl. im Literaturverzeichnis $z u$} & AG 70 & Svedova \\
\hline & $\mathbf{F}$ & Mann \\
\hline & Leitfaden & Voigt \\
\hline & $\mathbf{M}$ & Höck \\
\hline & MS & Miklosich \\
\hline & Sint.r.j. & Kubik \\
\hline & SS & Rùzicka 63 \\
\hline
\end{tabular}


I N H A L T

1. Kondensierte Strukturen als Beispiel für die Asymmetrie des sprachlichen Zeichens

1.1. Zur Korrelationsbeziehung zwischen russ. Partizip und Gerund

1.2. Ambiguität : Neutralisierung 3

1.3. Zu den Wortarten "Partizip" und "Gerund" 4

1.4. Zu Äquivalenzstrukturen 5

1.4.1. Aquivalenzstrukturen als konkurrierende 5

1.4.2. Äquivalenzstrukturen als Formen der Explikation

2. Zur Problematik syntaktischer Klassifizierungen

2.1. Zur paradigmatischen Ersatzprobe 10

2.2. Zu semantischen Implikationen 13

3. Zur Signifikanz der Oberflächenkriterien 19

3.1. Wortstellung 20

3.2. Isolierung 22

3.2.1. Zur Interdependenz mit der Korrelation 23

3.2.2. Variierung von Satzgliedpositionen $\quad 24$

3.2.3. Konstituierung von Satzgliedpositionen 27

3.2.4. Kommunikativ-semantische Signifikanz 28

3.3. Korrelation 31

3.3.1. Zur Definition für das Russische 33

3.3.2. Zur Realisierung $\quad 35$

3.3.3. Umwertung der aksl. Korrelation 38 
4.

Zum Begriff der Prädikation

4.1 .

Ebene des Satzes

44

4.1.1.

Morphologische Bestimung

45

4.1 .2 .

Syntaktische Bestimmung

47

4.1.3.

Kommunikative Bestimmung

4.2 .

Kondensierte Strukturen

52

4.2 .1 .

Semantische Bestimmung

55

4.2 .2 .

Logische Bestimmung

56

4.2 .3 .

Syntaktische Bestimmung

58

4.2 .4 .

Kommunikative Bestimmung

5.

Zur Relevanz der kommunikativen Intention

5.1 .

wahl kondensierter strukturen

5.2 .

Interpretation der Satzkonnexion

5.3 .

Konstituierung der sprachlichen Bedeutung

6.

Attributive Funktion

6.1

Zum Begriff des Attributs

6.2 .

Der funktionale Gegensatz restriktiv :

nichtrestriktiv und seine Kennzeichnung

6.2 .1 . Intonation

6.2 .2 .

Paraphrasen

6.2 .3 .

Transformationelle Beschreibung

6.3 .

Relativsatzparaphrase

6.4 .

Zum sprachlichen Material

94

6.4 .1 .

$P_{(1)}+\mathbf{N}$

6.4 .1 .1 .

Adjektivierung

6.4.1.2. Freie Stellung / Substantivierung

6.4.2.1. N+I $\mathrm{N}+\mathrm{P}+\mathrm{I}+\mathrm{V}$ 
6.4.3. $N+P_{1 / k}+V \quad 114$

6.4.3.1. N $N+P_{1}+V \quad 115$

6.4.3.2. N $\mathrm{N}+\mathrm{P}_{\mathrm{k}}+\mathrm{V} \quad 116$

6.4.4. $\mathrm{V}+\mathrm{N}+\mathrm{P}_{1 / \mathrm{k}} \quad 119$

6.4.4.1. $V+N+P_{1} \quad 119$

6.4.4.2. $V+N+P_{k} \quad 121$

7. Zur Struktur des Prädikatskomplexes 124

7.1. Zum relativen Tempus 124

7.2. Adverbiale Funktion in der binären $\begin{array}{ll}\text { syntaktischen Gliederung } & 127\end{array}$

7.2.1. Paraphrase durch adverbialen Nebensatz 128

7.3. Klassifizierung als "prädikativer

Determinant" 131

7.4. Signifikanz der Wortstellung 134

7.5. Differenzierung der traditionellen 138

7.5.1. "Syntaktische" Differenzierung 139

7.5.2. "Semantische" Differenzierung 142

7.6. Anwendung auf Partizipial- und

Gerundialstrukturen $\quad 149$

8. Funktionale Differenzierung des

Prädikatskomplexes - sprachliches Material 159

8.1. Anfangsstellung 159

8.1.1. Russ. Ger $+\mathrm{I}+\mathrm{N}+\mathrm{V} \quad 159$

8.1.2. Skr. Ger $+\mathrm{N}+\mathrm{V} \quad 162$

8.1.3. Aksl. $\mathrm{P}_{\mathrm{k}}+\mathrm{N}+\mathrm{V} \quad 163$

8.1.4. Russ. $P+I+N+V \quad 167$

8.1.5. Signifikanz der Anfangsstellung 171

8.1.6. Russ. Ger $+\mathrm{I}+\mathrm{V}+\mathrm{N} \quad 179$

8.2. Zwischenstellung 183

8.2.1. Russ. $N+I+$ Ger $+\mathrm{I}+\mathrm{V} \quad 185$ 
8.2.2. Skr. N + Ger + V

191

8.2.3. Poln. N+I+Ger + I+V

193

8.2 .4 .

Russ. $\mathrm{N}+\mathrm{I}+\mathrm{P}+\mathrm{I}+\mathrm{V}$

194

8.2.5.

Aksl. $\mathbf{N}+\mathrm{P}_{k}+\mathrm{V}$

200

8.2 .6 .

Russ. Ger $+\mathrm{I}+\mathrm{V}$

205

8.2 .7 .

Skr. Ger + V

208

8.2 .8 .

Aksl. $P_{k}+V$

208

8. 3 .

Endstellung mit Isolierung im Russischen

210

8.3.1.

Russ. $\mathrm{N}+\mathrm{V}+\mathrm{I}+\mathrm{Ger}$

214

8.3.2.

Russ. $\mathrm{V}+\mathrm{I}+\mathrm{Ger}$

223

8.3.3.

Russ. $\mathrm{V}+\mathrm{I}+\mathrm{Ger}+\mathrm{I}+\mathrm{N}$

225

8. 3. 4 .

Russ. $\mathrm{V}+\mathrm{N}+\mathrm{I}+\mathrm{Ger}$

226

8.3 .5 .

Russ. $\mathrm{V}+\mathrm{N}+\mathrm{I}+\mathrm{P}$

227

8.3.6.

Skr. postpositives Gerund

22 9

8.3 .7 .

Aksl. postpositives $P_{k}$

231

8.4. Endstellung ohne Isolierung im Russischen

238

8.4 .1 .

Aksl. gebundene Konstruktionen

238

8.4 .2 .

Skr. fokussierte Gerundien

243

8.4 .3 .

Russ. nichtisolierte strukturen

245

8.4.3.1. Koordinierung von Gerund und Adverb

8.4.3.2. Adverbialisierung?

8.4.3.3. Fokussierung

9.

Zusammenfassung

262

LITERATURVERZEICHNIS 
1 .

Kondensierte Strukturen als Beispiel für die

Asymmetrie des sprachlichen Zeichens

Kondensierte Strukturen veranschaulichen die asymetrische Struktur des sprachlichen Zeichens, die Notwendigkeit, zwischen den Ebenen des sprachlichen Ausdrucks und der Bedeutung zu unterscheiden und ihre nicht eineindeutige Zuordnung $z u$ untersuchen. 1 Die Partizipial- und Gerundialstrukturen des Russischen, die im Vordergrund unserer Uberlegungen stehen sollen, sind exemplarisch für diese Asymmetrie. Sie manifestiert sich in der Ambiguität dieser Formen und ihrer potentiellen Aquivalenz mit anderen Formen der Prädikation, wie sie auch in der Literatur zu Partizipialkonstruktionen anderer sprachen ${ }^{2}$ betont wird, ohne daß der Inhalt der Ambiguität/Äquivalenz immer präzisiert würde.

1.1. Zur Korrelationsbeziehung zwischen russ. Partizip und Gerund

Für das Russische ist ein wesentlicher Anhaltspunkt für die Bestimmung dieser Asymmetrie gegeben durch die Definition der Korrelationsbeziehung zwischen akt. Partizip und Gerund. ${ }^{3}$ Das merk-

1 Vgl.z.B. Zimmermann 75, S. 804: "Zur Problematik der Kondensation - Trotz des Reichtums der einzelnen Sprachen an Ausdrucksmitteln kann die Grammatik einschließlich des Lexikons ganz allgemein als ein Mechanismus der Verdichtung und relativen Verdunkelung von Bedeutungsfaktoren in sprachlichen AuBerungen charakterisiert werden. Der in diesem zusammenhang verwendete Begriff der Kondensation betrifft die unterschiedliche semantische Transparenz sprachlicher Äußerungen auf verschiedenen Strukturebenen."; vgl. auch Starikova 74, S. $14 \mathrm{ff}$ und die dort angefuhrte Literatur

2 Zur Ambiguität von lateinischen Partizipialkonstruktionen vgl. Heine 72, S. 217; zu deutschen nichtflektierten Partizipialkonstruktionen Bungarten 76, S. 32, 128ff, Rath 71, S. 127ff; zum Englischen Friederich 73; zum Aksl. Vełerka 61, S. 164; zum Poln. Weiss 77, S. 63, 84ff, 314ff; zum Russ. Kade 68, S. 604

3 Vgl. Rưzicka 62, Korrelationswandel, S. 685ff; ders. 62, Zur Rolle, S. $188 \mathrm{ff}$ 
mallose Partizip kann untergeordnet prädikativ und nichtprädikativ gebraucht werden; es tritt nicht nur im Rahmen des Nominalkomplexes, sondern auch im Prädikatskomplex auf. Das merkmalhafte Gerund signalisiert durch seine morphologische Form untergeordnete Prädikativität und (immer?-s.u.) Nichtzugehörigkeit zum Nominalkomplex. Das merkmallose Glied ist potentiell ambig und nur in einem Teilbereich seiner möglichen Bedeutungen äquivalent mit dem merkmalhaften Glied der Korrelation, nicht immer kann statt des merkmallosen Gliedes das merkmalhafte stehen.

Am sprachlichen Material ${ }^{4}$ ist die Realisierung der Korrelation zu untersuchen, konkret, ob sich im formal insbesondere durch Wortstellung und Isolierung $z u$ beschreibenden Kontext des Satzes für das merkmallose Glied Kriterien der Disambiguierung sowie der Äquivalenz zwischen Partizip und Gerund finden lassen. Grundsätzlich besteht die Möqlichkeit der Konkurrenz zwischen Partizip und Gerund im Sinne der asymetrischen Korrelation nur auf der Linie der Subjektsidentität mit dem satzschlieBenden Prädikat. Unsere Beispielsammlung beschränkt sich auf diese Fälle. 5

Neben der Zugehörigkeit zum Subjekts- oder Prädikatskomplex ist die innere struktur dieses Komplexes zu erörtern. Bevor hier von Ambiguität gesprochen werden kann, ist dazu die Eingrenzung der zugrundeliegenden semantischen Kategorien nötig. Im Rahmen des subjektskomplexes betrifft dies insbesondere den Gegensatz restriktiv : nichtrestriktiv und im Zusammenhang hiermit die Unterscheidung nichtprädikativer und untergeordnet prädikativer Funktion.

Für die untergeordnet prädikativen Partizipien und Gerundien. die gewöhnlich als dem Prädikatskomplex zugehörig beschrieben werden, ist $\mathrm{zu}$ unterscheiden $\mathrm{zwischen}$ Typen der Konnexion der untergeordnet prädikativen struktur mit dem Prädikat oder dem

4 Die Belege wurden der Sekundärliteratur entnommen; zur Kontrolle wurden einige Kapitel einer russischen tbersetzung von Thomas Manns "Dr. Faustus" mit ausgewertet (zitiert:F)

5 Zur "Subjektsidentität" vgl. 2.B. Mulisch 75, S. 206; Babby 75, S. 24; Kłízková 67, Problemy, S. 77 
Satzganzen und Varianten der Realisierung dieser Typen.

1.2. Ambiguität : Neutralisierung

Von Fällen der Ambiguität, wo immerhin einer nichteindeutigen Oberflächenstruktur eine Alternative funktionaler Möglichkeiten zuzuordnen ist, deren eine intendiert wird, sind Fälle der Neutralisierung semantischer Gegensätze zu unterscheiden. während im Hinblick auf Typen der Konnexion, wie sie z.B. RuZicka im Rahmen des Prädikatskomplexes beschreibt, die Möglichkeit der Ambiguität hinsichtlich funktionaler Unterschiede besteht, ist U.E. hinsichtlich der vielfach als "Varianten" der "adverbialen" Funktion aufgezählten temporalen, modalen, kausalen, konzessiven u.a.m. "Nebenbedeutungen" nicht von Ambiguität, sondern von Neutralisierung semantischer Unterschiede $z u$ sprechen, die im Falle von adverbialen Nebensätzen durch Konjunktionen expliziert sein können (aber nicht müssen - vgl. die Unbestimmtheit z.B. des deutschen indem). Es kann grundsätzlich eben nicht davon ausgegangen werden, die Partizipialoder Gerundialstruktur entspräche einem bestimmten adverbialen Nebensatz. Daraus ergibt sich, wie auch z.B. Weiss 77 mit Recht betont, ${ }^{6}$ daß, entgegen $2 . B$. Filipovid 77 , die Paraphrasierung durch Varianten adverbialer Nebensätze kein geeignetes Klassifikationskriterium für Partizipial- und Gerundialstrukturen ist, und letztlich auch, entgegen z.B. Babby 75, daß diese kondensierten Strukturen nicht von bestimmten Adverbialsätzen abgeleitet werden können, sondern allenfalls für kondensierte und explizite Oberflächenstrukturen eine gemeinsame zugrundeliegende

6 a.a.0.. S. 320: "Angesichts dieses Befundes wird man sich fragen muissen, ob nicht gerade diese semantische Unbestimmheit, die sich für Part-II-Konstruktionen als konstitutiv erweist. ihr pragmatisches Wesensmerkmal ausmacht: es spricht einiges dafür, daß der Sprecher die gerundiale Formulierung gerade deshalb wählt, weil er einer Präzisierung des semantischlogischen Bezugs aus dem Weg gehen will..."; vgl. Rùzicka 73. S. 459ff; zur Unbestimmtheit von Satzverknuipfungsrelationen vgl. auch Meyer 75, S. $43 f f$ 
Ausgangsstruktur anzunehmen ist. Dies hat weniger damit $z u t u n$, daß eine bestimme Information nicht mehr "auffindbar" ist in der kondensierten struktur, als damit, daß sie so möglicherweise gar nicht intendiert ist.

1.3. $\mathrm{Zu}$ den Wortarten "Partizip" und "Gerund"

Die Wortarten "Partizip" und "Gerund" sollen hier aufgrund ihrer morphologischen Kennzeichnung als gegeben vorausgesetzt werden: unser Interesse liegt nicht bei der Definition dieser "hybriden" Wortarten", sondern bei der Beschreibung von belegten Funktionstypen: Die tbergänge in andere Wortarten, so Adjektiv, Substantiv, Adverb, Präposition, können fließend sein. Unübersichtlich wird die Lage in der Sekundärliteratur insbesondere dann, wenn schon die Funktionsbestimmung der Partizipien und Gerundien selbst nicht ohne den Verweis auf diese verschiedenen Wortarten auskommt, oder doch jedenfalls nicht scharf genug getrennt wird zwischen wie immer zu definierender Wortart und möglichen syntaktischen Funktionen.

Ohne jeden weiteren Anspruch sei hier immerhin hingewiesen auf die Fähigkeit von russ. Partizipial- und Gerundialstrukturen zum Ausdruck relativer Zeitstufen ${ }^{8}$, die aber nicht immer realisiert sein muß, und auf die transformationelle Beschreibbarkeit durch einen in der Tiefenstruktur zugrundegelegten, durch Transformationen reduzierten Satz, - was aber nicht nur für diese Strukturen gilt, sondern z.B. auch für Infinitive. Schon an dieser stelle sei aber betont, daß sich das für die Definition der asymmetrischen Korrelation maßgebliche Merkmal der untergeordneten Prädikativität nicht auf eine Ableitungsgeschichte reduzieren läßt, insofern eben für in der Kommunika-

7 Vgl. zum Russ. Karcevskij 27, Jakobson 32, ders. 57, Jacobsson, Gö. 69, S. $23 \mathrm{ff}$, Jacobsson, Gu. 63, Rudnev 59, S. llff, Vinogradov 47, S. 384ff; zum Poln. Weiss 77, S. 59ff

8 Panzer 75, S. 119: "Für den Ausdruck relativer Zeitstufen stehen nur das Partizip und Gerundium des Präsens ( $p i \mathcal{Y}_{u} \boldsymbol{Z}_{\mathbf{Z}} \mathbf{j}$, vidja) für die Gleichzeitigkeit (in allen zeitstufen) und das Präteritalgerundium und perfektive Präsensgerundium (uvidev, uvidja) für die Vorzeitigkeit (in allen Zeitstufen) zur Verfügung." 
tion nichtprädikativ (z.B. Attribut) und für (untergeordnet) prädikativ intendierte strukturen in einer Tiefenstruktur letztlich allemal auf eine explizite Prädikation zurückgegriffen wird.

\subsection{Zu Äquivalenzstrukturen}

Der Begriff der Aquivalenz ${ }^{9}$ ist dem der Ambiguität komplementär. Das asymmetrische Verhältnis zwischen den Ebenen des sprachlichen Ausdrucks und der sprachlichen Bedeutung ist Voraussetzung dafür, daß für den sprecher die hahl besteht zwischen äquivalenten Formen sprachlichen Ausdrucks und für den Hörer die Wahl einer von mehreren möglichen Bedeutungen bei ambigem Ausdruck. 10

1.4.1. Aquivalenzstrukturen als konkurrierende Formen

Zu fragen wäre nach den Bedingungen der Wahl zwischen als äquivalent angenommenen strukturen, die demnach miteinander in Konkurrenz stehen. Noch wichtiger als die Tatsache, daß Synonymie im strengen Sinne 11 selten sein düfte, ja bestritten wird, ist, daß der Inhalt der Áquivalenz sich bisher einer exakten Definition entzieht. ${ }^{12}$ Er muß letztlich als durch die Intuition mehr oder weniger identifiziert vorausgesetzt werden; untersucht werden in Arbeiten zur Synonymie z.B. von Partizipialund Gerundialstrukturen gewöhnlich nicht identische Bedeutungs-

9 Zum Begriff der Äquivalenz: Ressel 77, Panzer 75, S. 185ff, Kononenko 70, insbes. S. 3-28, Suchotin 60, Conrad 69, S. loff, Leśka 66, S. 65ff, Apresjan 74

$10 \mathrm{Vgl}$. auch Petöfi 71; S. 254: "...daß die syntaktischen Strukturen in kommunikativ indifferenter Weise generierbar sind, die Frage der "Bedeutung" hingegen in dieser Weise nicht angegangen werden kann. Die Probleme der Bedeutung erheben sich für den"Hörer" anders als für den "Sprecher"."

11 Vgl. Immler 74, S. 109, Bungarten 76, S. 213

12 Vgl. Rư̌i ¿̌ka 73, S. 456; zur gen.Semantik Immler 74, S.165 
inhalte, sondern stilistische und grammatische Bedingungen des Gebrauchs der konkurrierenden Strukturen, nicht die Identität der Bedeutung, sondern Möglichkeiten der Wahl der sprachlichen Form. ${ }^{13} \mathrm{Z}$.T. wird lediglich eine ziemlich beliebige und unstrukturierte vielzahl von z.B. zu Partizipialund Gerundialstrukturen "synonymen" strukturen angefuhrt mit unterstellter Identität der Bedeutung und nicht weiter problematisierten Bedingungen der Wahl: vgl. insbesondere die übliche Ersetzung durch "Adverbialsätze" verschiedenster Art.

Immerhin zeigen die vielfachen Hinweise auf die Wahlmöglichkeit für den sprecher zwischen Partizipial- und Gerundialstrukturen und anderen "äquivalenten" Formen der Prädikation, auch wenn die Motive dieser wahl im einzelnen noch der Untersuchung bedurfen, daß eben der Gebrauch dieser Formen der Deprädikation hochgradig fakultativ ist, d.h. im Belieben der Intention des Sprechers steht.

1.4.2. Äquivalenzstrukturen als Formen der Explikation

Seitens des Hörers stellt sich nicht das Problem der Wahl zwischen potentiell äquivalenten strukturen, sondern der Interpretation von Ambiguitäten.

Ein wesentliches Hilfsmittel zur Explikation dieser Interpretation ist der Vergleich mit "äquivalenten" Zeichenstrukturen verschiedenster Art, die nicht in gleicher Weise ambig, sondern zumindest partiell eindeutig sind. Immer schon wurden im $\mathrm{Zu}-$ sammenhang mit Partizipial- und Gerundialstrukturen typologische Vergleiche natürlicher sprachen, Ubersetzungen in eine andere naturliche sprache sowie innerhalb der fraglichen sprache Paraphrasen und in letzter zeit Rückführung auf eine sei es mehr syntaktische oder logisch-semantische Form der Repräsentierung abstrakterer Art herangezogen, um Ambiguitäten der

13 Vgl. z.B. die Kritik von Kovtunova 55 , S.118ff an Francuk 52 
Ausgangsstruktur zu selektieren. ${ }^{14}$

Für die in unserem Zusammenhang als Diagnoseverfahren ${ }^{15}$ wesentlichen Paraphrasen und paraphraseähnlichen Tests wird dabei nicht völlige Identität der Bedeutung behauptet, sondern daß ihre Möglichkeit bzw. Unmöglichkeit und der Vergleich mit der Struktur, die sie ersetzen, Aufschlüsse liefert über deren funktionale Spezifika. Im Hinblick auf die Disambiguierung der Ausganqsstruktur heiBt dies insbesondere: AufschluB über die funktionstypkonstituierenden Voraussetzungen, unter denen der Sprecher die für den Hörer (bzw. den, der sprachliches Material zu untersuchen hat) ambige sprachliche Form gebraucht.

Im Ergebnis ist die Ersetzung einer kondensierten und ambigen Ausgangsstruktur durch eine (partiell) explizite Paraphrase der Versuch, die für die Ebene der semantischen Repräsentation

$14 \mathrm{Vgl}$. Weiss 77, S. $63 \mathrm{ff}$ (im Zusammenhang mit der Definition des Begriffs Partizip): "Hingegen eröffnet die Annahme zweier verschiedener Beschreibungsebenen, die in jüngster zeit immer mehr zur Repräsentation von Ausdrucks- bzw. Inhaltsstrukturen von Sätzen dienen, interessante Möglichkeiten für die Beschreibung polyfunktionaler Kategorien: was in der Oberflächenstruktur die Gestalt einer Kategorie $X$ annimmt, kann auf verschiedenen Tiefenstrukturen gründen. Von daher ließe sich das uns beschäftigende definitorische Problem vielleicht einer Lösung nahebringen: polnische Partizipialkonstruktionen wären dann Ketten, denen alternativ verschiedene Transformationsgeschichten der Art $T_{1}, T_{2} \ldots T_{n}$ zugrundeliegen: ob diese den in traditionellen Darstéllungen unterschiedenen Funktionen von PKs genau entsprechen, wird zu prüfen sein. Mit eben der skizzierten Methode in Zusammenhang steht eine weitere Möglichkeit der funktionalen Abgrenzung der Kategorie "Partizip": diese läßt sich charakterisieren durch Angabe ihrer Paraphrasebeziehungen, d.h. durch Auflistung jener Syntagmen, die sie ohne Veränderung des semantischen Gehalts ersetzen kann. Dieser zweite Weg, der ja schon in traditionellen Darstellungen beschritten wird, findet durch den ersten, die Angabe der $A b-$ leitungsgeschichten, einfach seine formale Explikation.": vgl. Danes u.a. 75, S. 616; Jakobson 74, S. 154-161; allgemein $z u$ Transformationen: Krenn 74, Rohrer 71, S. $46 \mathrm{f}$, Weiss 77, S. $52 \mathrm{ff}$, Prager Autorengruppe 75, S. $107 \mathrm{ff}$

15 Zum Begriff der "diagnostischen Transformation": Kade 68, Ruži đka 66, S. 48, Pfister 73, S. 314ff, Bungarten 76, S. $81 \mathrm{ff}$, S. $212 \mathrm{ff}$, Grepl 75, S. 662 , Vitek 67, S. 74; Zum Begriff der Paraphrase: Ungeheuer 68, Meyer 75, S. 63 
vorausgesetzte Eindeutigkeit $z u$ transponieren auf die Ebene der oberflächensyntaktischen Repräsentation und sie damit (wenn schon nicht $z$ u definieren) empirisch zugänglich zu machen. 16

16 Vgl. Bartsch 72, S. 21ff, 36; Sgall u.a. 73, S. 180, S. $242 \mathrm{ff}$ 
Sowenig auf die Ergebnisse der syntaktischen Tradition verzichtet werden kann, es muissen doch einige Hinweise zur Problematik syntaktischer klassifizierungen gegeben werden, die letztlich bedingt ist durch die asymmetrische struktur des sprachlichen Zeichens und anschaulich wird gerade in der Anwendung auf Partizipial- und Gerundialstrukturen.

Die Satzgliedpositionen sind heterogen, d.h. sie beruhen auf einer Mischungen formaler und semantischer Kriterien und sie haben insofern impliziten Charakter, als die zugrundeliegenden semantischen Merkmale der Satzgliedpositionen nicht definiert, sondern vorausgesetzt werden. ${ }^{1}$ Der Begriff der Semantik bleibt dabei unklar; er ist eher referentiell als sprachlich fundiert. Daß die maßgeblichen semantischen Beziehungen vorausgesetzt und nicht definiert werden, gilt auch noch für die klassische TG Chomsky'scher Prägung. Statt einer Definition der Funktion erfolgt Umschreibung anhand der Aufzählung möglicher Formen in den vorausgesetzten Positionen und durch Darstellung in einer Konfiguration, die ihre eigenen Voraussetzungen wiederholt und ihrerseits beruht auf dem vorausgesetzten, aber nicht explizierten Begriff des Satzes. 2

1 Vgl. Fedorov 72, S. $27 \mathrm{ff}, 31 \mathrm{ff}, 76 \mathrm{ff}$; Adamec 66, P.s.. S. 5, 11ff; Jäger 68, S. $32 \mathrm{ff}, 97 \mathrm{ff;}$ Hartung 73, S. 11ff; Raspcpov 70, S. 47ff; Bungarten 76, S. 82:

2 Vgl. Bartsch 72, S. $6 \mathrm{ff}, 18 \mathrm{ff}, 333 \mathrm{ff} ;$ Immler 74, S. 86ff, 110, 205ff; Rohrer 71, S. 16ff, 67ff; Lerot 70; Birnbaum 70, S. 9ff, 20ff; Coseriu 75, S. 126ff: "Es wird ausdruicklich von Chomsky bis $z$ u den letzten Vertretern der Transformationellen Grammatik betont, daß es kein Entdeckungsverfahren für sprachliche Fakten gibt, daß wir uns vielmehr auf die Intuition des native speaker verlassen mussen. Genau das führt aber $z u$ einer merkwirdigen und immer wieder $z u$ konstatierenden Zirkularität. Es wird einfach schematisch gezeigt: was das subjekt ist, ist sehr einfach, das ist die NP. die in der Tiefenstruktur links steht. Damit aber die NP gerade links und nicht rechts stehen kann, muß man wissen, daß dies die Funktion des subjekts besitzt.". 
Es soll an dieser Stelle der Hinweis genügen, daß sich aufgrund der Kritik an den traditionellen Satzgliedpositionen und der syntaktisch formulierten Tiefenstruktur bei vielen unterschieden im einzelnen in der Forschung doch mehr und mehr dahingehend Einigkeit abzeichnet, den Satzgliedpositionen eher oberflächencharakter zuzuschreiben, 3 die "Semantik als Basis der Syntax" 4 zugrundezulegen und die implizite und ambige oberflächensyntaktische Repräsentation zurückzuführen auf eine Ebene der expliziten semantischen Repräsentation.

Unser Interesse ist nicht so sehr modelltheoretischer Art; es soll deshalb nicht ausfuhrlich auf die Diskussion um den Begriff der Tiefenstruktur eingegangen werden, sondern lediglich kurz auf einige Probleme hingewiesen werden, die sich bei der Klassifizierung von (zumal fremdsprachigem) Material nach Satzgliedpositionen stellen.

2.1. Zur paradigmatischen Ersatzprobe

Die paradigmatische Ersatzprobe $z$.B. bei Bungarten 76 hat wesentlich die Funktion, die syntagmaüberschreitenden Beziehungen der (ambigen) Ausgangsstruktur im Sinne des vorausgesetzten syntaktischen Modells wiederum zu reduzieren auf die Domäne des Syntagmas und damit auf die Analogie $z u$ den klassischen Nebensatzgliedern. Es entspricht der logischen Tradition der syntaxforschung, einfaches Nebensatzglied, isoliertes Nebensatzglied (z.B. isolierte Partizipial- und Gerundialstrukturen) und Nebensatz als drei einander paradigmatisch zugeordnete mögliche Formen der Realisierung einer vorausgesetzten Satzgliedposition

3 Vgl. Prager Autorengruppe 75, S. 38ff, 84ff; Conrad 69, S. 10: Deszö/Szépe, S. 85 in Daneš 74 (ed.): Immler 74 S. 97ff; Weiss 77, S. 384ff; vgl, auch Adamcc, S. $192 \mathrm{ff}$ in Danes 74 (ed.): " Poverchnostnoe oformlenie predlozenij i poverchnostnye sintaksiceskie kategorii, takie, kak podležascee, skazuemoe, dopolnenie, opredelenie i.t.d.. predstavljajut soboj rezul'tat složnoj interakcii meźdu kategorijam urounja AC (= aktual'nogo Elenenija - Ch.H.) i kategorijam obobscenno-semanti ¿eskogo urounja."

4 Vgl. Panzer 75, S. 198; Immler 74, S. 110 
aufzufassen ${ }^{5}$ und angesichts der Ambiguität der Partizipialund Gerundialstrukturen anhand der paradigmatischen Ersatzprobe auf explizitere Realisierungen desselben Paradigmas zurückzugreifen. Die Interpretation der ambigen Ausgangsstruktur erfolgt dann durch Verweis auf die explizitere Struktur. Zwischen beiden besteht Paraphrasebeziehung bzw. Vermittlung durch "diagnostische Transformation". Dabei werden insbesondere intuitiv als attributiv/appositiv eingestufte Belege anhand der Ersetzung durch Relativsatz und "adverbiale" Belege anhand der Ersetzung durch adverbialen Nebensatz interpretiert. 6

Expliziert werden sollen auf diese Weise der Satzgliedstatus sowie zusätzliche semantische Varianten der Beziehung zwischen den Prädikationen durch die lexikalisch-semantische Spezifik der Konjunktion des adverbialen Nebensatzes.

Während man bei der Interpretation der Partizipial- und Gerundialstrukturen $z$ einem erheblichen Teil angewiesen ist auf Mutmaßungen hinsichtlich des kontextuellen zusammenhangs des Satzganzen, erlauben es diese expliziteren Paraphrasen, sich auf den Bereich des Satzes und innerhalb des Satzes auf den des Syntagmas $z$ u beschränken. Umgangen wird die Frage des syntagmaüberschreitenden "Doppelbezuges" auf subjekt und Prädikat und der untergeordneten Prädikativität selbst, insofern ja eine finitverbale, wenn auch syntaktisch untergeordnete Prädikation eingesetzt wird. Als "Beweis" für die Prädikativität der Ausgangsstruktur kann dies jedoch U.E. nicht dienen, gerade aufgrund der vorausgesetzten paradigmatischen Äquivalenz auch mit einfachem Nebensatzglied.

So nützlich diese an vorausgesetzten Satzgliedpositionen orientierte paradigmatische Ersatzprobe in praxi ist, es ergeben sich hier die gleichen Probleme, wie sie überhaupt gelten für die ungeklärten Voraussetzungen einer Definition der Satzglieder, so daß die Gefahr besteht, daß eine Unbekannte durch eine andere ersetzt wird und die Tatsache der Ersetzbarkeit verwechselt wird mit der Interpretation der Ausgangsstruktur, daß we-

5 Vgl. Panzer 75, S. 178ff; Rohrer 71, S. 189ff; Zolotova 73, S. 196ff; Prager Autorengruppe 75, S. $107 \mathrm{ff}$

$6 \mathrm{Vgl}$. Schaller 71; Filipović 77; Rath 71, S. 128ff; Schmidt, F. 57, S. 48ff; Bungarten 76, S. 81ff,90,212ff; Babby 75 
niger über den Inhalt der Aquivalenz und seine Konstituierung Auskunft gegeben wird als eben uber die syntaktische Funktion der eingesetzten Struktur ${ }^{7}$, deren "Äquivalenz" dann letztlich beruht auf der Identität des Denotats. 8

Das eigentliche Problem, die auch den Satzgliedpositionen zugrundeliegenden semantischen Beziehungen und deren Konstituierung, wird dabei als gelöst vorausgesetzt, und dies führt bei der Analyse von mehrdeutigem, zumal fremdsprachigem Material zu Schwierigkeiten.

Die Grenzen der paradigmatischen Ersatzprobe werden auch daran sichtbar, daß ja bei vorausgesetztem identischen Denotat Umwandelungen für Partizipial- und Gerundialstrukturen möglich sein können, die die vorausgesetzte syntaktische struktur verändern, insbesondere Umwandelung zu zwei selbständigen Sätzen oder Koordination zweier finitverbaler Prädikationen.

Nicht in jedem Falle kann eine logische Unterordnung von $\mathbf{z} . \mathrm{B}$. russischen Gerundialstrukturen im Sinne der traditionellen Nebensatzglieder vorausgesetzt werden. 9

Auch bei der Explikation des Satzgliedstatus können semantische Beziehungen ambig bzw. neutralisiert bleiben, die für die Ausgangsstrukturen als funktionstypkonstituierend angesehen werden können; dies betrifft den Gegensatz zwischen restriktiven und nichtrestriktiven Attributen bzw. Relativsätzen und z.B. das Problem des Wort-bzw. Satzbezuges auch im Fall expliziter Hypotaxen. Die traditionellen Satzglieder, insbesondere die "Adverbialbestimmung", sind keineeinheitlichen Kategorien, die sogenannten adverbialen Nebensätze ihrerseits weiterer Untersuchung bedirftig. 10

7 Vgl. Kovtunova 55, S. 118, 130ff; Heine 72, S. 220; Gabka 76. S. 214; Bungarten 76, S. 82

$8 \mathrm{Vgl}$. Rohrer 71, S. 92ff, 254ff; Coseriu 75, S. 111ff,127ff

9 Man vergleiche z.B. auch die Vielfalt der Ubersetzungsäquivalente $z u$ polnischen Gerundialkonstruktionen im Bulgarischen und Französichen bei Stankova 74; vgl. zu russ. Partizipialkonstruktionen Kornilov 67; Paffen II, $365 \mathrm{ff}$

$10 \mathrm{Vgl}$. kritisch $z$ u den "Adverbialnebensätzen" z.B. Hartung 73, S. 11, $13 \mathrm{ff}$ 
Die paradigmatische Ersetzung verschiedener Realisierungen einer vorausgesetzten Satzgliedposition durch einander kann nicht mehr AufschluB erbringen, als das zugrundeliegende syntaktische Modell an Differenzierungen enthält. Diese Hinweise implizieren, daß es nicht gerechtfertigt ist, finitverbale syntaktisch untergeordnete Prädikationen in der Form von Nebensätzen als den Partizipial- und Gerundialstrukturen im Sinne einer Reihenfolge von Ableitungsschritten $z u-$ grundeliegend anzusehen. ${ }^{11}$ Allenfalls kann die Möglichkeit der Paraphrasierung Indiz sein für einen gemeinsamen Ausgangspunkt der Ableitung.

\subsection{Zu semantischen Implikationen}

Die semantischen Implikationen, auf die sich auch die Arbeiten mit einer syntaktischen Terminologie beziehen, sind ihrerseits nicht hinreichend klar.

Dies zeigen die Diskussionen zur Unterscheidung von Attribut und Apposition im Rahmen der Nominalphrase ${ }^{12}$ sowie im Rahmen des Prädikatskomplexes die Auseinandersetzung um die Frage des zusammengesetzten : komplexen Prädikates 13 wie insbesondere um die verschiedenen Arten von Adverbialbestimmungen. 14 Die in zusammenhang mit Funktionstypen der Partizipial- und Gerundialstrukturen häufig angeführten Merkmale der untergeordneten Prädikativität, der Nichtrestriktivität : Restriktivität, der meist nicht näher bestimmten Fakultativität und des möglichen zweifachen semantischen Bezuges auf subjekt und Prädikat bzw. des Satzbezuges sind,auch wenn sie in syntaktisch

$11 \mathrm{Vgl}$. hierzu ausführlich Weiss 77, S. 52ff, $234 \mathrm{ff}, 356 \mathrm{ff}$; vgl. demgegenüber 2.B. Babby 75, S. 1: "I assume that these underlying embedded sentences are adverbial clauses (i.e. $[\mathrm{S}] \mathrm{AP}$. where "AP" is adverbial phrase), since this assumptioh captures the simple fact, long recognized by traditional Russian grammar, that the semantic range of gerunds is very close to that of adverbial clauses of time, reason. manner, etc."

$12 \mathrm{Vgl.z.B}$. Seiler 60

13 Vgl. 2.B. Kaלala 71

14 Vgl. z.B. Ressel 74 
orientierten Arbeiten wie z.B. bei Kaćala als funktionstypkonstituierend angeführt werden, zunächst einmal Merkmale semantischer Art, die syntaktisch z.T. nicht erfaßbar, vor allem aber U.E. irrelevant sind im Sinne eines traditionellen Satzgliedschemas, das den Satz in die beiden Hauptsatzglieder Subjekt und Prädikat zerlegt und für diese die Möglichkeit der Erweiterung in der Form von Syntagmen durch jeweils einseitig untergeordnete Nebensatzglieder verschiedenen Umfangs vorsieht. Dieses Satzgliedschema entstammt der logischen Grammatiktradition und gilt, in abgewandelter Formulierung, bis heute. Sein Nutzen soll nicht bestritten werden; in unserem Zusammenhang ist jedoch wesentlich, daß für Partizipialund Gerundialstrukturen Beziehungen auftreten, die den Bereich des Syntagmas überschreiten. 15

Sichtbar wird die hier vorliegende Problematik, wenn z.B. Ruzićka 66 und Kt1zková 69 zwar in umschreibenden Formulierungen den Bezug des "sekundären Prädikats" auf das Satzganze nahelegen, in der Konstituentenstruktur aber doch nur Unterordnung unter den dem Subjekt gegenüberstehenden Prädikatskomplex vorsehen.

Es entsteht die mißliche situation, daß die oben angegebenen semantischen Merkmale, die für die Definition der traditionellen Satzglieder keine Rolle spielen, bei der Klassifizierung von Partizipial- und Gerundialstrukturen nach diesen Satzgliedern nur als zusätzliche Merkmale, die die vorausgesetzte syntaktische Funktion variieren, angefuihrt werden, andererseits aber doch konstitutiv sind für die spezifik der Funktion dieser strukturen.

Reflex dieser Schwierigkeiten ist die bis anhin inkonsistente Begründung des Merkmals der untergeordneten Prädikativität durch semantische Kriterien und durch die formalen Kriterien der Isolierung und der Wortart, die vielfältigen Benennungen

15 Vgl. z.B. Michnevid 68, S. 106ff; Valgina 72: Kornilov 64 Bungarten 76, S. $128 \mathrm{ff}$ mit ausführlichem Hinweis insbesondere auf die untergeordnete Prädikativität, Fakultativität/Nichtrestriktivität und syntagmaüberschreitende semantische Beziehungen der deutschen Partizipialkonstruktionen 
und Definitionen des umstrittenen Satzgliedes "sekundäres Prädikat" und seine fließerde Abgrenzung gegenüber den traditionellen Satzgliedern Apposition und adverbiale Bestimmung.

Die Unubersichtlichkeit dieser Situation verweist letztlich auf das Nichtausreichen der syntaktischen Ebene des Syntagmas und des Satzes für die funktionale Spezifizierung z.B. der Partizipial- und Gerundialstrukturen. Die Möglichkeit der Erfassung der genannten semantischen Merkmale hängt ab vom zugrundegeleqten linguistischen Modell. Die Schwierigkeiten einer syntaktischen Darstellung liegen darin, daß ad hoc Anleihen genommen werden bei anderen Funktionsebenen, hier U.E. insbesondere der Ebene der in der Kommunikation konstituierten und mitgeteilten sprachlichen Bedeutung. 16

Das heißt: Die fortbestehenden Schwierigkeiten einer Klassifizierung der Partizipial- und Gerundialstrukturen ${ }^{17}$ z.B. des Russischen resultieren wesentlich nicht nur aus der vielfach erwähnten Ambiguität der Form selbst, sondern auch aus der unbewältigten Notwendigkeit. Kategorien und Konstituierung von semantischen Beziehungen überhaupt präzise $z u$ formulieren. Als relevant für die Konstituierung von Funktionstypen werden die verschiedensten Gesichtspunkte angefürt. 18

Es soll natürlich nicht bestritten werden, daß im Einzelfall eine Vielzahl möglicher Faktoren zusammenwirkt, angefangen $z$. $B$. vom Vorwissen des Sprechers und Hörers über die außersprachliche wirklichkeit und den sprachlichen und situationellen Kon-

16 Zum Begriff der sprachlichen Ebene: Immler 75, S. 56ff, a.a.O. S. 58: "Eine neue Ebene der Sprachbeschreibung wird immer dann notwendig, wenn es sprachliche Erscheinungen gibt, die auf den bisher angenommenen Ebenen nicht beschrieben werden können." a.a.0. S. 61ff: "Die Aufstellung einer neuen, höheren Ebene der Repräsentation wird immer dann notwendig, wenn ein bestimmter Typ von Regeln nicht ausreicht oder nicht angemessen ist, um die Erscheinungen der Sprache $z u$ beschreiben: $d . h$. eine sprachliche Ebene trennt zwei Bereiche, in denen verschiedene Typen von Regeln herrschen."

$17 \mathrm{Vgl}$. Rath 71, S. 127ff: "Es ist nun eines der schwierigsten Kapitel in der Semantik, eine systematische und vollständige Aufstellung dieser allgemeinen kategoriellen semantischen Beziehungen zu geben. Dies ist bislang noch nirgends befriedigend gelungen.": Rư̌z.,SS,S.56,159; ders.71, Festschr.f.J.Kurz,S. 306

18 Rúzička 73, S. $459 \mathrm{ff}$ 
text uber die lexikalische Semantik der beteiligten Komponenten, ihre syntagmatische stellung, wortart, morphologische Merkmale bis zur aspektual-temporalen Funktion.

Die Schwierigkeit ist nur, daß, so sehr derartige Hinweise für das einzelne Beispiel treffend sein mögen, die Vermittlung zwischen diesen Einzelgesichtspunkten und der syntaktischen Klassifizierung, die im Ergebnis angewendet wird, weitgehend fehlt. Es fehlt gerade im Fall der hochgradig ambigen und fakultativen Partizipial- und Gerundialstrukturen an einer systematischen Unterscheidung von das Einzelbeispiel kennzeichnenden zusätzlichen Merkmalen und strukturbildenden systematisierbaren Kategorien.

Bungarten 76 z.B. nennt als maßgeblich für die Entscheidung iber attributive : adverbiale Funktion deutscher Partizipialkonstruktionen die wortstellung, deren Bedingungen nicht untersucht werden, und außersprachlich begründete semantische pelationen. 19 Der Bereich des außersprachlich Bezeichneten entzieht sich, wie auch Bungarten betont, der linguistischen Analyse; er ist nicht $z$ u erschließen aus der sprachlichen Form und findet offenbar seine widerspiegelung in den Beziehungen, die resultieren sollen aus der semantischen Charakteristik der beteiligten Lexeme. 20 Der Untersuchung zugänglich, insbesondere anhand der paradigmatischen Ersatzprobe durch Relativsatz bzw. adverbialen Nebensatz ist nur das syntaktische Ergebnis dieser, wie Bungarten unterstellt, außersprachlichen Bedingungen. An dieser Stelle wird der referentiell begründete Äquivalenzbegriff der an Satzgliedern orientierten paradigmatischen Konzeption nochmals deutlich. Kritisiert werden soll dabei nicht das syntaktische Ergebnis der Klassifizierung, nur: dies ist keine Begründung für die angenommenen Funktionstypen. 21 wortstellung und außersprachlich begrüdete semantische Relationen stehen

$19 \mathrm{Vgl}$. Bungarten 76, S. 32, 42, 128ff, 160ff, 226

20 a.a.O.. S. $157 \mathrm{ff}, 173 \mathrm{ff}, 268$

21 Hinsichtlich des außersprachlich bezeichneten Sachverhaltes besteht doch kein Unterschied zwischen den folgenden Sätzen: Paula ist schön; sie arbeitet in der Fabrik/Die schöne Paula arbeitet in der Fabrik/Paula arbeitet in der Fabrik, obwohl sie schön ist.: vgl. auch Kł́́źková 69, PO, S. 9: "Po vo osnovnom èkvivalentno neobosoblennomu nerestriktivnomu atributu". 
unvermittelt nebeneinander und sind nicht im sinne einer zeichenrelation aufeinander $z u$ beziehen.

Die These, daß die letztlich referentiell begrüdete lexikalisch-semantische Charakteristik der beteiligten Lexeme die Funktionstypen der Partizipial- und Gerundialstrukturen konstituiere, findet sich vielerorts, ohne daß sie eigentlich begrundet wirde: 22 so auch in der $A G$ 70, wo allgemein auf die funktionstypkonstituierende Relevanz von aspektual-temporalen Beziehungen und der lexikalischen Semantik der beteiligten Komponenten hingewiesen wird, ohne daß ein zusammenhang mit dem Merkmal der Isolierung und der durch sie signalisierten untergeordneten Prädikativität und der in deutlicher Analogie zu den konventionellen Satzgliedern gegebenen Klassifizierung der isolierten Wortarten hergestellt würde.

Es ist $z u$ betonen, daß $j a$ nicht die beteiligten Lexeme, sondern die Beziehungen zwischen ihnen in Frage stehen, und daß sehr wohl bei gleichem Bestand an Lexemen verschiedene Beziehungen möglich sind, sowohl hinsichtlich der zuordnung zum subjektsoder Prädikatskomplex als auch hinsichtlich der vielen Varianten der sogenannten adverbialen Funktion (temp., kaus.. mod.., usw.).

Hinreichend präzise Formen der Beschreibung vorausgesetzt, wäre das Problem der Vieldeutigkeit hinsichtlich dieser Beziehungen weitgehend gegenstandslos, ließe sich die Beziehung zwischen den Lexemen von diesen selbst ableiten. Die Implizitheit und Ambiguität hinsichtlich der Beziehungen läßt sich nicht auf die Ambiguität der Lexeme reduzieren, wie die Unbestimmtheit hinsichtlich sogenannter adverbialer Varianten zeigt. Die Fragwïdigkeit der lexikalisch-semantischen Orientierung wird anschaulich bei der Untersuchung deutscher Partizipialkonstruktionen bei Filipovic 77. Hier werden den Typen und Varianten von Beziehungen semantische Gruppen von zugrundeliegenden Verben statistisch zugeordnet, ohne daß U.E. ein Zusammenhang evident

$22 \mathrm{Vgl}$. 2.B. Kaćala 69, S. 709; Schrenk 68, S. 175, 237: Belić 69 II, S. 192; Večerka 61, S. 118, 161ff; Grochowski 75. S. $673 f f ;$ AG 70, S. 645 
wïrde. Im einzelnen ergeben sich strittige Interpretationen; es wird nicht immer präzis unterschieden zwischen der Semantik der Komponenten und der der Beziehung;insbesondere wird die Unterscheidung hinsichtlich attributiver : adverbialer Interpretation vorausgesetzt und durch Paraphrasen expliziert, aber gerade nicht von Lexemen abgeleitet.

Die Skepsis z.B. von Rath, Rúzicka, Bungarten, Weiss ${ }^{23}$ geqenuber den Möglichkeiten einer semantischen Klassifikation der fakultativen und ambigen Partizipial- und Gerundialstrukturen begründet sich wesentlich durch die Aussichtslosigkeit, aus den beteiligten Lexemen erschlossene semantische strukturen des außersprachlich Bezeichneten systematisch sprachlichen Formen zuzuordnen und letztlich durch die fortbestehende Unklarheit hinsichtlich der funktionstypkonstituierenden Faktoren, die eine Zuordnung von sprachlichem Ausdruck und Bedeutung im Sinne der asymmetrischen Zeichenrelation verhindert. Es ist nicht klar, was eigentlich an "Bedeutung" den Oberflächenstrukturen zuzuordnen sein soll.

23 Weiss 77, S. 315ff "Paraphrasierung als semantische Klassifikationsmöglichkeit von Part-II-Konstruktionen?" 
Die Oberflächenkriterien, in unserem Falle insbesondere Wortstellung, Isolierung und Realisierung der Korrelation sind nicht eindeutig hinsichtlich der Satzgliedpositionen: ${ }^{1}$ außerdem -dies ergibt sich aus den ungeklärten semant1schen Implikationen der für Partizipial- und Gerundialstrukturen angeführten Satzgliedpositionen- ist nicht klar zu unterscheiden, welche Oberflächenkriterien wann relevant sein sollen für die Identifizierung von Satzgliedern und wann Oberflächenkriterien als Varianten von identifizierten Satzgliedern anzusehen sind. In praxi hat die ungeklärte Signifikanz dieser kriterien oft zur Konsequenz, daß vorausgesetzte Satzgliedpositionen eineindeutig formalen Kriterien zugeordnet, im Grunde auf diese reduziert werden: ${ }^{2}$ man vergleiche hierzu die Interpretation des russischen Partizips als ausschließlich attributiv z.B. bei Babby 75. Ahnlich sieht Rudnev das isolierte Partizip ausschließlich als "opredelenie", nennt aber zusätzliche semantische Merkmale, die dann doch wieder Affinität zur prädikativen Adverbialbestimmung herstellen.

Angesichts der Nichteindeutigkeit formaler Merkmale ist es unumgänglich, auf semantische Beziehungen zurückzugreifen bei der Bestimmung der fuir Partizipial- und Gerundialstrukturen relevanten Satzglieder.

Dies führt z.B. bei Rúzicka dazu, daß er intuitiv plausible Funktionstypen weniger syntaktisch bestimmt, wie es Rủzickas Ansatz entspräche, als durch Beispiele und "etwas außerhalb der Syntax" durch Hinweise zur Semantik dieser Strukturen erläutert; ${ }^{3}$ in der syntaktischen Tiefenstruktur werden die unterschiedlichen Positionen weniger definiert als vorausgesetzt

1 Vgl. Raspopov 70, S. 56ff; Bungarten 76, S. 42, 81ff,90,165

2 Vgl. Rúżǐ̌ka 66, S. 27 zur Apposition, S. 41, 54ff zum "sekundären Prädikat"

3 Vgl. die Formulierung bei Rủžička 66, S. 48: (zu semantischen Beziehungen, die) "wenigstens teilweise auch syntaktisch expliziert werden können"; vgl. a.a.O. S. 54ff; vgl. die Skepsis gegenüber den Differenzierungen Rúzičkas bei Weiss 74 . S. 193, ders. 77, S. 341ff; vgl. auch Panzer 75, S. 177ff 
und durch Hinweis auf (nichteindeutige) Oberflächenkriterien motiviert.

\subsection{Wortstellung}

Die Relevanz der Wortstellung ${ }^{4}$ des Russischen auch für die syntaktische Gliederung veranschaulichen die folgenden Beispiele; der Gegensatz der Wortstellung unterscheidet hier die zugehörigkeit zum Subjekts- oder Prädikatskomplex:

Cudnoe utro bylo / Utro bylo cudnoe

Smuscennyj i rastrevozennyj brat vernulsja/ Brat vernul-

s ja smuscennyj $i$ rastrevozennyj

Doktor-geolog / Geolog-doktor

Tempy $v$ epochu rekonstrukcii resajut vse / Tempy rełajut vse vejpochu rekonstrukcii / v épochu rekonstrucii tempy resajut vse

(nach Buttke 69, S. 55ff)

Dabei ist es nicht unser Thema, das Spannungsverhältnis zwischen der sogenannten normalen, grammatischen Wortstellung und der durch den Kontext bedingten zu erörtern. ${ }^{5}$ Allerdings treten auch Sätze mit "normaler" Wortstellung ja gewöhnlich nicht isoliert, 2.B. in Beispielsammlungen, auf, sondern im durch Kontext und Situation bestimmten kommunikativen zusammenhang; dieser ist wirksam für die Anwendung auch der "grammatischen" Wortstellung.

Von der syntaktischen wird die kommunikativ-semantische Signifikanz ("aktuale Gliederung") der Wortstellung im Russischen

4 Zur syntaktischen Funktion der Wortstellung im Russischen: Adamec 66, P.s.. S. $12 \mathrm{ff:}$ : Fedorov 72, $31 \mathrm{ff}, 77$

5 Vgl. z.B. Ebeling 58: AG 70, S. $596 \mathrm{ff}$ und die Kritik von Bucharin 73; vgl. auch Firbas in Danes 74 (ed.), $S .36$ : "Grammatical structure can become the leading factor within the hierarchy of word order principles, but only on condition it duly serves the communicative purpose of the utterance, not infringing the requirements of FSP." 
und in anderen Sprachen unterschieden. ${ }^{6}$ sie steht in Interdependenz mit der Satzintonation einschließlich möglicher Pausen ("Isolierung"). 7

Fuir die hochgradig fakultativen und ambigen Partizipial- und Gerundialstrukturen z.B. des Russischen dürfte eine exakte Unterscheidung zwischen syntaktisch und kommunikativ-semantisch bedingter Wortstellung schwierig sein. Zunächst einmal läßt sich nur die weitgehende Beweglichkeit der Wortstellung ${ }^{8}$ für Partizipial- und Gerundialstrukturen feststellen und die grundsätzliche funktionale Relevanz der Wortstellung. Wie man ihre Signifikanz bewertet, hängt ab vom zugrundegelegten linguistischen Modell.

Bungarten 76 nennt $^{9}$ für nichtflektiertes, isoliertes, fakultatives, untergeordnet prädikatives deutsches Partizip mit Subjektsidentität im wesentlichen die folgenden Wortstellungstypen, anhand derer "attributive" und "adverbiale" Funk$t i o n$ unterschieden werden:

$\mathrm{N}+\mathrm{I}+\mathrm{P}+\mathrm{I}+\mathrm{V}^{10}:$ attr

$\mathrm{V}+\mathrm{N}+\mathrm{I}+\mathrm{P}: \operatorname{att} \mathrm{r} / \mathrm{adv}$

$\mathrm{N}+\mathrm{V}+\mathrm{I}+\mathrm{P}: \operatorname{adv}$

$\mathrm{P}+\mathrm{I}+\mathrm{V}+\mathrm{N}: \mathrm{adv}$
Sie aber, zwischen dem Bräutigam und dem 2 willingsbruder sitzend, griff, zwischen den Stuhlen, nach dessen Hand (Bung. .S.152)

Dann sab sie lange auf der Bettkante, grübelnd und sinnend daruber, wie es nun weitergehen sollte

(Bung..S.161-adv)

eine zeit lang gibt es noch Herden, weidend am Rand des möglichen Lebens

(Bung..S.163 - attr)

Die Lösung... unternimmt Kant, ausgehend von der mathematischen Naturerkenntnis... (Bung..S.57)

ausgehend von der mathematischen Naturerkenntnis..., unternimm kant die Lösung (Bung..S.57)

6 Vgl. Panzer 75, S. 146; Adamec I 73, S. 128ff, 131ff, ders. 66. P.s.: Raspopov 70, S. 30ff, 133ff, Pumjanskij 74

7 Adamec 66, P.s.. S. 17ff, Rudnev 56, S. 25ff; Rưzićka 65 , Verbalphr., Anm. 18

8 Zum Poln. Weiss 77, S. 329ff; zum Aksl.Rưzicka,63, SS, S. $85 \mathrm{ff}, 135 \mathrm{ff}$

$9 \mathrm{Vgl}$. Bungarten 76, S. $268 \mathrm{ff}$

$10 \mathrm{~N}=$ Nomen/Subjekt, $\mathrm{V}=$ Verbum/Prädikat, $\mathrm{P}=$ Partizip, I=Isolierung 
Fiir attributive Interpretation ist in jedem Fall Bedinaung N+I+P. ${ }^{11}$ zugrunde liegen dem syntaktisch benannten Gegensatz attributiv : adverbial semantische Relationen, 12 deren Fundierung unklar bleibt. Die grundsätzliche Signifikanz der wortstellung für diese semantischen Relationen relativiert U.E. die von Bungarten angenommene Relevanz außersprachlicher und lexikalisch-semantischer Gesichtspunkte. In diesem zusammenhang sind die Beobachtungen Bungartens zur je nach Funktionstyp tendenziell unterschiedlichen "syntaktisch-semantischen Qualität des Akteurs"13 von besonderem Interesse, wonach z.B. bei attributiver Funktion ein Pronomen als subjekt so gut wie ausgeschlossen ist. Damit ist uber die Grenze des Satzes hinaus verwiesen auf die unterschiedliche textuelle Stellung des attributiven und des adverbialen Funktionstyps.

Die syntaktische Klassifizierung erscheint als Ergebnis von Bedingungen, die uber die syntaktische Ebene des Satzes hinausreichen, aber innerhalb des Satzes ihren Reflex finden, u.a. im Oberflächenkriterium der Wortstellung.

\subsection{Isolierung}

Das formale Kriterium der Isolierung soll hier provisorisch als durch die Interpunktion gegeben vorausgesetzt werden. Dies ist eine grobe Vereinfachung, ausfürliche Untersuchungen zum Verhältnis von intonatorischer Gestaltung des Satzes in der Rede und Interpunktion wären nötig. Es ist mit Fällen zu rechnen, in denen, insbesondere bei Nichterweiterung des durch Komma abgetrennten Nebensatzgliedes, in der gesprochenen Rede keine Pause gemacht wird, und wo umgekehrt eine sprechpause nicht durch Interpunktion signalisiert wird.

11 Vgl. Bungarten 76, S. 173; Rath 71, S. 76

$12 \mathrm{Vgl}$. Bungarten 76, S. 66, 157ff, 268

$13 \mathrm{Vgl}$. Bungarten 76, S. 166ff; zur Interdependenz von Pronominalisierung und wortstellung im Russischen vgl. z.B. Křižková, Synt. ch.. S. $121 \mathrm{ff}$ 
3.2.1. Zur Interdependenz mit der Korrelation

In Interdependenz mit der Wortstellung ist die Isolierung ein wesentliches strukturelles Merkmal des Russischen, dessen Relevanz, auch im typologischen Vergleich, eng verknüpft ist mit der Entwicklung der asymetrischen Korrelation ${ }^{14}$ zwischen akt. Partizip und Gerund, konkret mit der Möglichkeit für das akt. Partizip, bei Isolierung auch untergeordnet prädikativ zu fungieren und damit in Konkurrenz $z$ u treten mit dem Gerund, dies im Unterschied z.B. zum Poln..$^{15}$, Cech.'. Skr. oder zum Aksl., wo die Langform des akt. Partizips ausschließlich nichtprädikativ gebraucht wird.

Im Skr. besteht keine Korrelation zwischen akt. Partizip und Gerund, vielmehr wurde das Partizip entweder adjektiviert oder ersetzt durch Relativsatz. Ubrig bleibt nach Verlust der Flexion das untergeordnet prädikative Gerund ${ }^{17}$, also gegenüber dem Aksl. das merkmallose Glied, im Vergleich mit dem Russischen das merkmalhafte; zur Signalisierung der untergeordneten Prädikativität reicht die morphologische Form, die Isolierung ist allenfalls ein zusätzliches Grenzsignal, jedenfalls ist es nicht strukturell verankert durch die Möglichkeit der untergeordnet prädikativen Funktion des (ambigen) Partizips wie im Russischen. Am Material ist zu untersuchen, wie weit der Isolierung in Interdependenz mit der Wortstellung für das skr. Gerund Signifikanz zukommt.

Im Poln. z.B. existieren zwar akt. Partizipien, jedoch nicht in Korrelation $z u$ den Gerundien, sondern nur in Opposition.

14 Georgieva 68, S. 99ff: Rużička 63, Typ., S. 841

$15 \mathrm{Vgl}$. Weiss 77, S. $377 \mathrm{ff}$

$16 \mathrm{Vgl}$. auch Rúzička 62, Korr., S. 685: "Das syntaktische System der modernen tschechischen Literatursprache nutzt auf Grund seiner stärker verbalen, weniger nominalen Anlage die Isolierung weit weniger zur Signalisierung syntaktischer Gliederungsverhältnisse, vor allem der peripheren Prädikativität, als die russische Literatursprache."

$17 \mathrm{Vgl}$. Pesikan 59, S. 96ff; Leskien 14, S. 554ff, 558ff: Vaillant 31, S. $253 \mathrm{ff}$; Belić $69 \mathrm{II}, \mathrm{S} .188 \mathrm{ff}$; zum Verlust der Kongruenz vgl. M, S. $1-19$ und die dort angefürte Literatur (s. Lit.verz. unter Höck, Ch.: Untersuchungen...) 
d.h.: eine funktionale tberschneidung von Gerundial- und akt. Partizipialstrukturen ist nicht gegeben: ${ }^{18}$ die Verwendung pass. Partizipialstrukturen z.B. als "Apposition" im Sinne von Weiss $77^{19}$ soll hier nicht erörtert werden. Bei Koordinierung mit Adjektiv allerdings ist auch für akt. Partizip sekundärprädikative Funktion möglich. Vgl.:

Z natury łagodny i nie szukajacy konfliktów, tym razem jednak nie mogzem sig opanowán (Weiss 77, s. 380)

Grundsätzlich reicht die morphologische form aus zur signalisierung des Unterschiedes zwischen nichtprädikativer (attributiver) und sekundärprädikativer Funktion, die Isolierung ist nicht von grundlegender Relevanz zur Unterscheidung möglicher Funktionstypen des Partizips. Dem entspricht auch die im Poln. (und Ćech.) durchaus übliche Distribution $N+P$, während im Russischen $\mathrm{N}+\mathrm{I}+\mathrm{P}$ bei weitem uberwiegt und die strukturelle Voraussetzung liefert für $\mathrm{P}+\mathrm{I}+\mathrm{N}+\mathrm{V}$ mit sekundärprädikativer Funktion des akt. Partizips.

Im Aksl. steht zur Signalisierung der untergeordnet prädikativen Funktion nur die Kurzform des akt. Partizips zur Verfügung; die Isolierung, soweit aus der Interpunktion der Texte $z$ u erschließen, ist als zusätzliches Mittel der Gliederung von Interesse, nicht jedoch distinktiv für die Interpretation des potentiell ambigen Korrelationsgliedes, bei dem prädikative Funktion bei weitem dominiert.

\subsubsection{Variierung von Satzgliedpositionen}

Die Interpretation der russischen Isolierung in der Forschungsgeschichte ist charakteristisch für die jeweils zugrundeliegende syntaktische Konzeption. ${ }^{20}$ Die Vorläufigkeit des Diskussions-

$18 \mathrm{Vgl}$. Weiss 77, S. 59ff, 377ff, 380ff

$19 \mathrm{Vgl}$. Weiss 77, S. $227 \mathrm{ff}, 352 \mathrm{ff}$

20 Nicht zutreffend ist die Annahme bei Bungarten 76, Anm. 359 . wonach Faulseit und Kühn 63 (?) den Terminus "Isolierung" geprägt hätten, wie ja schon ein Blick auf die Arbeiten... 
standes wird deutlich in der Vielfältigkeit der Bedingungen, die maßgeblich sein können für Isolierung bzw. in der Vielfalt der Funktionen der Isolierung. 21 so nennt z.B. Kolšans$\mathrm{kij} 62$, S. $35 \mathrm{ff}$ die folgenden formalen Bedingungen der Isolierung:

"1. morfologičeskie osobennosti opredeljajuscego slova

2. morfologiそeskie osobennosti opredeljaemogo slova

3. sostav opredeljajuščej gruppy i ee rasprostranennost'

4. mestopoloženie opredeljajušc̉ej gruppy

5. sosedstvo drugich opredelenij

6. nekotorye drugie grammaticeskie faktory" Rudnev 68, S. $128 \mathrm{ff}$ nennt als Funktionen der Isolierung:

"1. vydelit' prisuščie opredeljaemym licam, javlenijam, predmetam priznaki osoboj znacimosti...

2. pojasnit', utoćnit', konkretizirovat', raskryt' smysl' togo ili drugogo klena predloženija ili ego leksiceskogo vyraženija...

3. usilit' smyslovoe ili emocional'noe soderźanie drugogo Łlena predloźenija protivopostavlenija, sravnenija i.t.p....

4. pokazat' osobye sintaksičeskie svjazi obosoblennoj gruppy slov $v$ predloženii ili dvojnuju sintaksičeskuju, a sledovatel'no i smyslovuju funkciju togo ili drugogo člena predloženija..."

Die übersichtlichste Form der Darstellung ergibt sich, wenn man auf der Grundlage der logischen Tradition der Syntaxforschung nebenrangige Satzglieder (insbesondere Attribut, Adverbialbestimung) voraussetzt und ihnen als Paradigma möglicher Realisierungen einfaches nichtisoliertes Satzglied, isoliertes Satzglied und Nebensatz zuordnet. 22 Damit entsteht ein Par-

20 ... Rudnevs und die $\mathbf{z} . \mathrm{B}$. dort angefühte Literatur zeigt: $\mathbf{z u r}$ Ubersicht uber die Forschungslage z.B. Rudnev 59, S. 7ff, 28 , ders. 63, S. $132 \mathrm{ff}$, ders. 68, s. 120ff, Ananic 72, S. 26ff, Georgieva 68, S. $98 \mathrm{ff}$, Bukatevic 58 (ed), S. $313 \mathrm{ff}$, Gvozdev II73, S. 152ff,Žáa 64, Kolšanskij 62, S. $36 \mathrm{ff}$

$21 \mathrm{Vgl.z.B.} \mathrm{Dozorec} \mathrm{72;} \mathrm{Gnevko} \mathrm{75,} \mathrm{S.} \mathrm{71ff;} \mathrm{Rudnev} \mathrm{59,} \mathrm{S.56ff}$

22 Vgl. 2.B. Gabka 76, S. 214; Żà̇a 64; ders. 65 
allelismus isolierter und nichtisolierter nebenrangiger satzglieder, die Isolierung erscheint als oberflächenkriterium der Variierung einer vorausgesetzten und identifizierten Satzgliedposition, dessen Signifikanz unschrieben wird mit dem Hinweis auf größere semantische Selbständigkeit, untergeordnete Prädikativität u.ä.. d.h. auf zusätzliche funktionale Merkmale, die nicht konstitutiv sein sollen für die Satzgliedposition und deren status in der schwebe bleibt. ${ }^{23}$ Dies gilt auch für die zusätzlichen Schattierungen adverbialer Bedeutung und den Bezug auf Subjekt und Prädikat gleichzeitig, die für isolierte Partizipial- und Gerundialstrukturen genannt werden. 24 Die Unsicherheit hinsichtlich der strukturellen Bewertung der Isolierung zeigt sich z.B. bei Kŕizková ${ }^{25}$ darin, daß sie dieses Oberflächenkriterium auch noch in der Konstituentenstruktur auffüht zur Unterscheidung der Varianten "Prädsec" und "AdvComit", die innerhalb des Prädikatskomplexes in identischer Position angefuhrt werden, sich aber unterscheiden durch Paraphraseverhalten und stellung in der aktualen Gliederung. Für das slk. sieht demgegenüber Kacala $71^{26}$ Isolierung bzw. Nichtisolierung des "doplnok" ausschließlich als Performanzerscheinung der Oberfläche ohne strukturelle Relevanz.

$23 \mathrm{Vgl.}$. B. Panzer 75, S. 177: "Hier handelt es sich nicht um neue Satzglieder im Sinne einer neuen semantosyntaktischen Funktion, sondern eher um die schon bekannten, besonders Attribute (incl. Apposition) und Umstandsbestimmungen, in einer syntaktisch und intonationsmäßig selbständigen, halbprädikativen Funktion"; a.a.O. S. 178: "Es fragt sich aber doch, ob hier nicht eine bloße oberflächensyntaktische Struktur der bekannten Satzglieder (Attribut, Circumstantial) vorliegt, wie es auch bei vielen Nebensätzen der Fall ist."; vgl. für das Deutsche auch Bungarten 76, S. 133, 208; zum Russischen: Rudnev 68, S. 36, 146ff, 151ff, Ruzicka, SS, S. 23; zu "Nexus"-Strukturen vgl. auch Birnbaum 60, S. 45ff, $55 \mathrm{mit}$ Verweis auf Jespersen;

24 Svetlik 70, S. 265ff; Gnevko 75, S. 71; Valgina 72, S. 99, Sintaksis $r . j .74$, S. looff, $136 \mathrm{ff}$; Pićugov $73,5.33 \mathrm{ff}$ : Ananic 72, S. 27ff; Rudnev 68, S. $153 \mathrm{ff}$

$25 \mathrm{Vgl}$. Kŕižková 69, Po: dies. 69, Synt. ch.

$26 \mathrm{Vgl}$. Kačala 71, S. $101 \mathrm{ff}$ 
3.2.3. Konstituierung von Satzgliedpositionen

Kolšanskij 27 sieht die Isolierung nicht als formales Mittel zur Variierung vorausgesetzter Satzgliedpositionen, sondern zur Konstituierung syntaktischer Beziehungen wie Kongruenz und Rektion. Kolšanskij selbst begründet seine Ablehnung des "sekundären Prädikats" als eigenes Satzglied gerade mit der syntaktischen Signifikanz der Isolierung. Eine Auseinandersetzung hierüber ist solange erschwert, als die auch von Kolsanskij angenommenen zweitrangigen satzglieder ihrerseits nicht definiert sind. Rùzicka betont ebenfalls die syntaktische Relevanz der Isolierung und zieht eben hieraus die Konsequenz, das obligat isolierte "sekundäre Prädikat" als eigenes Satzglied zu etablieren.

Vgl. zur syntaktischen Relevanz der Isolierung Rưzićka 65 , Skizzen, S. 76, Anm. 18:

"Die sogenannte Isolierung (obosoblenie) ist in der modernen russischen Literatursprache eine syntaktische Tatsache ersten Ranges. Sie hebt z.B. zahlreiche Regeln der Juxtaposition auf, oder anders gesagt: zahlreiche Juxtapositionen von Phrasen sind nur mit Isolierung möglich. Der Gegensatz Isolierung : Nichtisolierung kann bei gleicher Wortfolge mit Unterschieden der Konstituentenstruktur verbunden sein, die in dem Phrasenstrukturteil nicht dargestellt werden können. Die transformationelle Beschreibung wird vor allem den RegelmäBigkeiten der Isolierung bei der Einbettung der im Quellensatz nach dem Kopula-Verbum byt' stehenden prädikativ-nominalen Elementen nachzugehen haben."

Die konstituierende Relevanz der Isolierung für die Satzgliedposition (wie immer deren Funktion dann zu bestimmen ist) zeigen Beispiele für den Distributionstyp $\mathrm{P}+\mathrm{I}+\mathrm{N}+\mathrm{V}$ :

Ustalye i izmucennye putniki totcas że usnuli/ Ustalye i izmučennye, putniki totkas ze usnuli

Je nachdem, welche Differenzierungen man innerhalb des Prädikatskomplexes vorsieht (z.B. PrádN/Advb/Prădsec bel Ružićka 66), denen jedenfalls nach Ružickka strukturelles Gewicht zukommen soll, ist die Isolierung auch innerhalb des Prädikats-

27 Vgl. Kolśanskij 62, S. 34ff; Rưzíćka 63, Typ., S. 841 
komplexes als distinkitv $2 u$ werten, insofern Prädsec obligat isoliert ist, Advb isoliert sein kann und PrädN obligat nichtisoliert ist.

Bei Kolłanskij und bei Rużicka ist, wenn auch mit unterschiedlicher Konsequenz, ein erster Schritt in Richtung auf eine Auflösung des Parallelismus vorausgesetzter Satzglieder, die isoliert und nichtisoliert auftreten können, getan, insofern eben die Isolierung selbst syntaktisch relevant wird, im Unterschied zur oben angefürten Bewertung der Isolierung als zusätzlichem Oberflächenkriterium zur Variierung vorausgesetzter satzgliedpositionen mit nur provisorisch umschriebener semantischer Signifikanz, das im Grunde syntaktisch irrelevant ist.

Die angedeuteten Schwierigkeiten hinsichtlich konstituierender/ variierender Funktion der Isolierung für Satzgliedpositionen werden zunächst vermieden, wenn, wie in der AG 70, S. 645ff, nicht mehr im ersten schritt auf Satzgliedpositionen zurückgegriffen wird, sondern nur Wortarten genannt werden, die isoliert auftreten können und infolgedessen in der Außerung mit relativer semantischer selbständigkeit (Halbprädikativität) fungieren. Sehr viel ist allerdings auch hiermit nicht gewonnen, denn vom allgemeinen Hinweis auf die kommunikative Sonderstellung isolierter Wendungen abgesehen, geht die Analogie zwischen isolierten und nichtisolierten Funktionstypen so weit. daß im einzelnen fraglich wird, ob die Isolierung zusätzliches oder konstituierendes Merkmal für die Klassifizierung der angefürten Funktionstypen ist und die kommunikative Spezifik dieser strukturen undeutlich bleibt.

\subsubsection{Kommunikativ-semantische Signifikanz}

Isolierung wird also z.T. interpretiert als formales Kriterium zur Signalisierung von Satzgliedpositionen,z.T. zur Variierung von vorausgesetzten Satzgliedpositionen. Die in diesem zusammenhang angeführten semantischen Merkmale verweisen über die syntaktische Ebene (deren semantische Implikationen dabei ungeklärt bleiben) hinaus in den Bereich der kommunikativ-semanti- 
schen Gliederung der ÄuBerung. Vgl. z.B. AG 70, S. 643:

"Obosoblenie ili neobosoblenie togo ili inogo grammatiCeski organizovannogo smyslovogo otrezka v sostave predloženija predopredeljaetsja zadačami vyskazyvanija. Obosoblenie slovoformy ili gruppy slovoform vsegda svjazano tem, čto obosoblennyj otrezok vnutri predloženija aktualiziruetsja, priobretaet otnositel'nuju smyslovuju samostojatel'nost' ili neset $v$ sebe ottenok dopolnitel'nogo soobšcenija. Obosoblennye slovoformy ili gruppy slovoform nazyvajutsja polupredikativnymi. $V$ sostave predlozenija mozet obosobljat'sja ne ljubaja iz vchodjaścich $v$ ego sostav slovoform ili grupp slovoform. Zdes' dejstvujut opredelennye ogranicenija, kasajusčiesja preżde vsego komponentov strukturnoj schemy predlozenija, kotorye nikogda ne mogut obosobljat'sja ni sami po sebe, ni vmeste s grammatićeski zavisjaśćimi ot nich slovoformami."

Vgl. Rudnev 68, S. 123:

"Obosoblennym nazyvaetsja takoj vtorostepennyj klen predlozenija, kotoryj izpol'zuetsja $v$ reci dlja usilenija, pojasnenija libo utočnenija smyslovoj roli, svjazi ego s opredeljaemym Clenom predloźenija $i$ vydeljaetsja pri proiznosenii i na pis'me."

Der kommunikativ-semantischen Ebene und nicht der syntaktischen sind die Merkmale zuzuordnen, die in Zusammenhang mit der Isolierung angeführt werden, wie untergeordnete Prädikativität, Nichtrestriktivität, Bezug auf das Satzganze. 28 so beschreibt 2.B. Zaża 64, ähnlich Kŕizková, die Isolierung in Interdependenz mit der Wortstellung als Kriterium der aktualen Gliederung $^{29}$ und sieht den Gegensatz restriktiv : nichtrestriktiv, signalisiert durch die Isolierung, als durch die aktuale Gliederung konstituiert.

Auch die formalen "Bedingungen" der Isolierung, wie z.B. Pronominalisierung des subjekts, Umfang der isolierten Gruppe und Wortstellung haben ihrerseits als Bedingung nicht die syntaktische Gliederung des Satzes, sondern dessen Stellung im

28 Kŕizková 69, Po, S. Sff; Zaza 64, S. 185: Sviblova 62: Pi Cugov 73, S. 33ff, 36; Adamec II 75, S. 9ff, 30, 170: Ananic 72, S. 40; Georgieva 68, S. 96ff

$29 \mathrm{Vgl}$. Sergeeva 73; Lapteva 72, S. 35; Valgina 72, S. 100; Krylova 70; Valimova 71; zum Deutschen Seiler 60, S. 19; zum Französischen Banaru 75, S. 26 
Zusammenhang des Textes und der Situation; sie sind nicht "Ursache" der Isolierung, sondern zusammen mit ihr der Reflex kommunikativer Zusammenhänge im Satz; ${ }^{30} \mathrm{bzw}$. wenn man sie als Bedingung der Isolierung auffaßt und die Isolierung als syntaktisches Kriterium anfuhrt wie Kolsanskij und Rủzicka, ist daraus zu schließen auf die Konstituierung von syntaktisch benannten Funktionstypen der Partizipial- und Gerundialstrukturen im Satz durch Bedingungen des Kontextes als dessen kommunikativ-semantische Gliederung ("aktuale Gliederung" u.ä.).

Die ublichen syntaktischen Klassifizierungen von isolierten Partizipial- und Gerundialstrukturen stehen vor der Schwierigkeit, daß die semantischen Implikationen der Nebensatzglieder nicht definiert sind. Die funktionalen spezifika der Partizipial- und Gerundialstrukturen (vgl. "untergeordnete Prädikativität") sind nicht syntaktischer Art; die in diesem zusammenhang relevanten formalen Kriterien der Wortstellung und Isolierung werden angefüht zur Identifizierung und zur Variierung syntaktischer Positionen, ohne daß ihre Signifikanz expliziert würde.

Statt nichtexplizierte semantische voraussetzungen und formale $\mathrm{Kri-}$ terien ad hoc $z u$ vermischen, ${ }^{31}$ soll versucht werden, am Material zu unterscheiden zwischen nichteindeutigen oberflächenstrukturtypen und semantischen strukturen und diese zuzuordnen.

So provisorisch diese Uberlegungen notwendigerweise sind, Grundlage für diese zuordnung ist die These, daß die formalen Kriterien Wortstellung und Isolierung signifikant sind für Strukturen der in der Kommunikation konstituierten (einzel-) sprachlichen Bedeutung. 32

$30 \mathrm{Vgl.z}$.B. Ananič 72, S. 31ff, Sintaksis r. j., s. 100ff; Rudnev 68 , S. $127 \mathrm{ff}, 143 \mathrm{ff}$

31 Im Zusammenhang mit "primykanie" u.a. von russ. Gerundialkonstruktionen vgl. Svetlík 70, S. 30: "Resajuscuju rol" pri etom vide sintaksićeskoj svjazi igraet smyslovaja svjaz' zavisimogo slova s gospodstvujušcim slovom, ego mesto $v$ predloźenii (porjadok slov) i intonacija."

32 Zur Signifikanz von Wortstellung und (u.a.) Isolierung für die "aktuale Gliederung" " "kommunikativ-syntaktische" Ebene usw. vgl. 2.B. Raspopov 70, S. $133 \mathrm{ff}, 148 \mathrm{ff:}$ vgl. auch Dezsö/Szepe, S. $81 \mathrm{ff}$ in Danes 74 (ed.) 
Vgl. Dezsö/Szépe, S. 88 in Dahl 74 (ed.):
"It has turned out -at least for us- that the introduc- tion of the communication pattern may be suitable for the analysis of syntactic phenomena more or less dis- regarded in formal linguistics so far, such as the stress and pause. They and the word order can be exa- mined as a unit if we analyze the communicational topic- comment relations too."

Die syntaktischen Klassifizierungen der Partizipial- und Gerundialstrukturen orientieren sich entweder nur an Oberflächenkriterien oder beruhen auf einer subjektiven Mischung verschiedener Funktionsebenen. Die Integration der Ebene der in der Kommunikation konstituierten sprachlichen Bedeutung in ein linguistisches Modell ist noch nicht abschließend gelungen. Argumente wenigstens dafür, daß dies notwendig ist, lassen sich allerdings gerade am Beispiel der hochgradig ambigen und fakultativen Partizipial- und Gerundialstrukturen anfüren.

\subsection{Korrelation}

Die Konsequenz aus der nichteindeutigen zuordnung von sprachlicher Form und sprachlicher Bedeutung ist die Konzeption der asymetrischen Korrelation, wie sie die Beziehung zwischen russischem akt. Partizip und Gerund beschreibt; d.h.: es ist $z u$ unterscheiden zwischen dem hinsichtlich einer bestimmten Bedeutung merkmalhaften Glied, das diese Bedeutung eindeutig bezeichnet, und dem merkmallosen Glied, daß diese Bedeutung in Interdependenz mit dem Kontext allenfalls ausdrücken kann, aber nicht muß, also potentiell ambig ist. Die Nichtspezifizierung hinsichtlich eines Merkmals eröffnet zugleich die Möglichkeit zur Spezifizierung hinsichtlich anderer Merkmale. 33

33 Zum Begriff der asymmetrischen Korrelation vgl. Jakobson 32, S. 74: "Falls die Kategorie I das Vorhandensein von A ankündigt, so kündigt die Kategorie II das Vorhandensein von $A$ nicht an, d.h. sie besagt nicht, ob $A$ anwesend ist oder nicht. Die allgemeine Bedeutung der Kategorie II im Vergleich zu der Kategorie I beschränkt sich auf den..... 
Grundsätzlich besteht im Russischen die Möglichkeit zur Äquivalenz $\mathrm{zwischen}$ merkmalhaft untergeordnet prädikativem Gerund und merkmallosem Partizip nur auf der Linie der Subjektsidentität mit dem satzschließenden Prädikat. Für immerhin ca. ein Drittel der von Gö. Jacobsson untersuchten russischen Partizipien liegt Subjektbezug vor. ${ }^{34}$ Nur derartige Fälle sollen hier von Interesse sein.

Dem russischen untergeordnet prädikativen Gerund entspricht im Aksl. die nominativische Kurzform des Partizips. Vgl. zum Aksl. Ružicka, ss, S. 238:

"Die getrennte Behandlung der Partizipien des Nominativs und der aller anderen Kasus ist schon deshalb berechtigt und notwendig, weil nur nominativische Partizipien den Weg zum Gerund einschlagen konnten und damit ihre Sonderstellung deutlich bekunden.

Worin diese Sonderstellung besteht, läßt sich deshalb am klarsten an der syntaktischen Eigenart der Gerundien, nämlich ihrer Prädikativität erkennen. Der Bezug auf ein mit dem Prädikat gemeinsames Subjekt, die Abhängigkeit von dem an der spitze der syntaktischen Hierarchie des Satzes stehenden und selbst nicht abhängigen subjekt und die relative Selbständigkeit, die sich daraus für den Fall ergeben kann, daß kein Subjekt vorliegt, all das verleiht der potentiellen Prädikativität des Partizips größten Spielraum.

Das nominativische Partizip liegt durch den gemeinsamen Subjektbezug auf der gleichen syntaktischen "Linie" wie das Prädikat und kann deshalb vorzugsweise peripheres Prädikat sein; denn in den Grenzen des einfachen Satzes sind es -von absoluten Konstruktionen abgesehen- uberhaupt nur Handlungen des gleichen subjekts, die in ein direktes Verhältnis zueinander gesetzt werden können. Ein prädikatives Verhältnis der Handlungen ungleicher Subjekte kann nur zusammen mit einem kasuellen Abhängig-

33 ... Mangel der "A-Signalisierung". Falls in einem bestimmten Kontext die Kategorie II das Nichtvorhandensein von A ankündigt, so ist es bloß eine der Anwendungen der gegebenen Kategorie: die Bedeutung wird hier durch die situation bedingt" S. 83: "Die merkmallose Form fungiert im sprachlichen Denken als Repräsentant des Korrelationspaares."; vgl.a.a.0. S.82ff; Barnet 65. S. 161, 176ff; Panzer 75, S. 95, zu den Termini "ausdruicken" und "bezeichnen" a.a.0.S. 121

$34 \mathrm{Vgl}$. Jacobsson, Gö. 69, S. 46ff, 125ff, 128: zur Ambiguität von akkusativischen Partizipialkonstruktionen hins. Prädikativität/Nichtprädikativität vgl. z.B. Rúzicka, SS, S. $238 \mathrm{ff}$, $243 \mathrm{ff}, 359 ;$ M. . S. 71ff: 
keitsverhältnis bestehen.

Aber es ist nicht nur diese komplexe grammatische Beziehung des nichtnominativischen Partizips, die dem nominativischen seine Vormachtstellung verleiht: auch in semantischer Hinsicht bieten sich in erster Linie Handlungen des gleichen Subjekts zur Verbindung peripherer und zentraler Prädikation an."

3.3.1. Zur Definition für das Russische.

Allerdings ist bis in jüngste Zeit die Möglichkeit der funktionalen Uberschneidung von russischem Partizip und Gerund nicht allgemein anerkannt.

So sieht z.B. Jacobsson 69 für Partizipien attributive, appositive, substantivische, prädikative Funktion vor, für Gerundien adverbiale. ${ }^{35}$ Diese Untersuchung ist vorwiegend orientiert an der statistischen Verteilung von oberflächenkriterien. Nur Gerundien werden hinsichtlich ihrer Beziehung zum Prädikat erortert, nicht aber Partizipien. Eben dies wäre Voraussetzung für die Erörterung der möglichen Synonymie von Partizip und Gerund. Die von Jacobsson angenommene "grammatical synonymy" für Partizip und Gerund bei Subjektsidentität betrifft nicht deren Funktion, sondern eher den Bereich des außersprachlich Bezeichneten.Vgl. die Beispiele bei Jacobsson 69, S. 46:36 vystupavij na zasedanie sekretar' predstavil tesisy... sekretar', vystuparsij na zasedanie, predstavil tesisy... vystupaja na zasedanie, sekretar' predstavil tesisy... (Vgl. mit ähnlichen Unterstellungen zur "Synonymie" Kade 68. S. 612.)

In jedem Fall unmöglich ist der Gebrauch des Gerund bei Bezug auf Nomen im obliquen Kasus:

ja dumaju o sobytijach, proischodjascich v... (Jacobsson 69, S.46) Babby $75^{37}$ z.B. ordnet den morphologischen Gegensatz zwischen

$35 \mathrm{Vgl}$. Jacobsson, Gö. 69, S. $41 \mathrm{ff}, 43 \mathrm{ff}, 52 \mathrm{ff}, 55 \mathrm{ff}$

36 Gemeinsam ist Partizip und Gerund hier allenfalls die Möglichkeit der Rückführung auf einen "Quellensatz" mit identischem subjekt

$37 \mathrm{Vgl}$. auch Kanšin 56; Gvozdev I 73, S. 377ff, 386ff; Mulisch 75, S. 193 
Partizip und Gerund eindeutig "attributiver" und "adverbialer" Funktion zu. Die Plausibilität der von Babby angeführten Bejspiele und ihrer Explikation durch Relativsatz/adverbialen Nebensatz soll nicht bestritten sein; so sehr allerdings die Beschreibung der Funktion des Gerund zutreffen mag, damit ist noch nicht ausgeschlossen, daß auch das Partizip in dieser Funktion auftritt. Entgegen Babby ist nicht einzusehen, warum Sätze des Typs

rodivšajas' za granicej, moja mat' plocho govorit po-anglijski mit nichtattributivem Partizip grundsätzlich ungrammatisch sein sollen. 38

Neben der strikten Trennung der syntaktischen Funktion fuir Partizip und Gerund finden sich auch Klassifizierungen, die zwar terminologisch unterscheiden, jedoch in den mehr oder weniger semantischen Umschreibungen dieser Funktionstypen, insbesondere im Zusammenhang mit der Signifikanz der Isolierung, immerhin die Möglichkeit funktionaler Affinität erkennen lassen. So führt z.B. Rudnev bei isoliertem Gerund ('obosoblennoe pred. obstojatel'stvo") untergeordnete Prädikativität und adverbialen Bezug auf das Prädikat an und ebenso für das isolierte Partizip ("obosoblennoe opredelenie"), insbesondere in Anfangsstellung. Die morphologische Form des Partizips gibt hier Anlaß zur Klassifikation als Attribut, die semantische Signifikanz der Wortstellung und Isolierung wird analog $z$ Gerundialstrukturen umschrieben. 39

Dementsprechend werden z.B. in der AG 70, bei Kŕiźková 69, bei Adamec II 75 und bei Ruzicka untergeordnet prädikative Partizipialstrukturen explizit auch außerhalb des Subjektkomplexes erörtert; d.h.: das merkmallose akt. Partizip des Russischen kann nichtprädikativ (attributiv) innerhalb des subjektkomplexes auftreten und untergeordnet prädikativ innerhalb des Prädikatskomplexes ("sekundäres Prädikat" z.B. bei Rưžicka, prädikatives Attribut $=$ doplněk = vtoroe skazuemoe u.a.m. bei

38 Zum Distributionstyp $\mathrm{P}+\mathrm{I}+\mathrm{N}+\mathrm{V}$ ausführlich Rủžicka 73: vgl. auch Rúzicka 66, S. 50; ders. 59, Struktur, S. 545

$39 \mathrm{Vgl}$. Rudnev 59, S. 25ff, $44 \mathrm{ff}, 58,64 \mathrm{ff} ;$ Bukatevic 58 (ed.), S. $313 \mathrm{ff}$; Isaćenko 75, S. $324 \mathrm{ff}, 334 \mathrm{ff}$; Leitfaden 71, S. 107ff, 151ff; Gabka 75, S. $216 \mathrm{ff}$ 
Adamec II 75). Das merkmalhaft untergeordnet prädikative Gerund signalisiert schon durch seine morphologische Form die Nichtzugehörigkeit zum Subjektskomplex und wird syntaktisch als sekundäres Prädikat usw. klassifiziert.

Das Gerund deckt nur einen Teilbereich möglicher Funktionen des Partizips ab, dieses ist ambig, zur Spezifizierung seiner Funktion angewiesen auf den Kontext, der innerhalb des Satzes formal insbesondere durch die Kriterien der Wortstellung und Isolierung $\mathrm{zu}$ beschreiben ist, und partiell synonym mit dem Gerund. Aus der morphologischen form ist die syntaktische Klassifizierung des Partizips nicht vorhersagbar. Konkret: die bekannte Relativsatzparaphrase gilt, entgegen z.B. Babby, nicht für alle Distributionstypen des Partizips, z.B. nicht für alle $N+I+P$ in $N+I+P+I+V$ oder $V+N+I+P$ (s.u.) und in keinem Fall bei $\mathrm{P}+\mathrm{I}+\mathrm{N}+\mathrm{V}$; infrage kommt statt dessen u.a. adverbialer Nebensatz mit Konjunktion sowie die Ersetzung durch Gerund.

\subsubsection{Zur Realisierung}

Nach der allgemeinen Definition der Korrelationsbeziehung zwischen russischem akt. Partizip und Gerund wäre zu fragen nach den Bedingungen der Realisierung der Korrelation, d.h. nach den Typen der Distribution, nach den Bedingungen der Wahl zwischen potentiell äquivalentem Partizip und Gerund und andererseits nach Kriterien der Disambiguierung des potentiell mehrdeutigen (merkmallosen) Partizips. Die Definition der Korrelation nennt nur Möglichkeiten der Funktion, notwendige Bedingungen für die Verwendungen der Korrelationsglieder, spezifiziert aber nicht hinreichend die jeweils vorliegenden Funktionstypen. Einschränkungen hinsichtlich der morphologischen Bildbarkeit von Gerundien ${ }^{40}$ sollen dabei nicht erörtert werden in unserem Zusammenhang, sondern es soll versucht werden, im

$40 \mathrm{Vgl}$. Rứicka 62, Zur Rolle, S. 189 
Material vorgefundene Funktionstypen zu spezifizieren.

Rủucka $66^{41}$ setzt schon für die Ebene der Tiefenstruktur folgende Positionen als unterschieden voraus, in denen Partizipial-und Gerundialstrukturen auftreten können:

- Partizip tritt auf innerhalb des Subjektskomplexes als Apposition und im Prädikatskomplex als "sekundäres Prädikat" (abzuleiten über Gerund)

- Gerund im Rahmen des primären Prädikatskomplexes als "Advb" und außerdem als sekundäres Prädikat.

Das heißt: Durch einander ersetzbar sind Partizip und Gerund hier in der Position "Prädsec", insbesondere im Distributionstyp Part/Ger+I+N+V.

Dabei weist Rûzicka nicht explizit hin auf das Nichtauftreten des Partizips als "Advb" innerhalb des primären Prädikatskomlexes, dies ergibt sich lediglich aufgrund der angefühten Beispiele, wie überhaupt Rủźicka (und Kade) die Funktionstypen weniger definiert als vorausgesetzt und anhand von Beispielen. Paraphrasen und Konstituentenstrukturen veranschaulicht haben. Rúźićka 73, S. $45 \mathrm{ff}$ sieht den Unterschied zwischen beiden Korrelationsgliedern in der Funktion des sekundären Prädikats $z u-$ nächst einmal in der Ableitungsgeschichte: Partizip geht zurück auf einen Satz mit Prädikatsnomen, Gerund auf das finitverbale Prädikat eines zugrundeliegenden Satzes. Insofern ist das Partizip in noch höherem Maße als das Gerund kondensiert. Das Partizip soll in solchen Fällen die Bedeutung einer generischen Nominalphrase haben, für Gerundien der Spielraum bei der Belegung der Konnektorvariablen größer sein als für Partizip. Vgl.:

legko spravljajuścijsja so vsemi zadanijami, na étot raz ućenik ne mog resit' zadacil

$41 \mathrm{Vgl}$. Rủzicka 66, S. 27ff, 36, 42, 47ff; ders. 65, Verbalphr.. ähnlich Kade 68, der allerdings auch noch Gerundien im Rahmen des subjektskomplexes beschreibt; vgl. $2 u$ Rúzicka die kritischen Hinweise insb. zur Transformationsgeschichte bei Kŕíḱková 69, Synt. ch., S. $116 \mathrm{ff:}$ vgl. auch die Kritik bei Weiss 74, s. 188ff, ders. 77, s. $341 \mathrm{ff}$ 
legko spravljajas'so vsemi zadanijami, na étot raz urenik ne mog resit'zadadi (Rúzicka 73, S. 457) Kŕizková 42 unterscheidet annäherend analog $2 u$ Rứićka innerhalb des Prädikatskomplexes zwischen (nichtisoliertem) AdvComit und (isoliertem) Prädsec. Normal tritt in diesen Positionen das Gerund auf, Partizip nur selten und allenfalls als Prädsec, solange die "Handlungsbedeutung" des Partizips erhalten ist und nicht Adjektivierung eintritt.

Soweit aus den Beispielen der AG 70, S. $643 f \mathrm{fu}$ ențehmen ist, tritt hier isoliertes Gerund als (prädikatnähere) adverbiale Bestimmung auf und als Satzdeterminant, das isolierte Partizip jedoch, anders als bei Ružička, als adverbiale Bestimmung. In diesem Zusamenhang mag der Hinweis auf die Zuordnung von Endungstypen zu syntaktisch-semantischen Funktionstypen von Partizipien in russischen Dialekten nach Kuz'mina-Nemćenko 71 von Interesse sein. 43

Es werden unterschieden prädikative, halbprädikative (predikativnyj atribut/predikativnoe obstojatel'stvol und nichtprädikative (atribut/obstojatel'stvo) Funktion.

Prädikative und prädikativattributive Funktion unterscheiden sich durch den Charakter des ibergeordneten Verbs (Kopula: syn-/autosemantisch). Gemeinsam ist, daß der Prädikatskomplex als semantische Einheit mit Endstellung des Partizips fungiert und daß beide Male sämtliche aufaezählten Endungstypen auftreten können.

Demgegenüber fungieren pred. obstojatel'stvo und obstojatel' stvo beide als Erweiterung der prädikativen Basis, beide benützen die gleiche Teilmenge an Endungen des aufgezählten Inventars, der Unterschied soll liegen in der Angabe eines Merkmals als Prozeß (pred. obst.) bzw. als zustand (nichtpräd. obst.)

Je nach Nähe zum zentrum der Prädikation besteht also unterschiedliche Wahlmöglichkeit innerhalb des Endungsinventars:

42 Kŕižková 69, Po, S. 12, 33ff; dies. 69, Synt.ch., S.115ff, $119 \mathrm{ff}, 122 \mathrm{ff}$

$43 \mathrm{Vgl}$. Kuz'mina/Nemćenko 71, S. 8ff, 306ff (Tabelle) 
der lexikalisch-semantische Charakter des finiten Prädikats und die semantische Charakterisierung des Partizips (Zustand: Prozeß) ist hierfür nicht letztlich entscheidend. Die Verteilung der Endungstypen läßt sich so zusammenfassen, daß kongruierende und akongruente Formen prädikativ und prädikativ-attributiv aufreten, akongruente in der Funktion als (predikativnoe) obstojatel'stvo.

Die Definition der asymmetrischen Korrelationsbeziehung zwischen Partizip und Gerund reicht zur spezifizierung möglicher Funktionstypen noch nicht aus. Die untergeordnet prädikative Funktion von Partizipialstrukturen wird unterschiedlich begründet und impliziert keine vollständige Synonymie mit untergeordnet prädikativen Gerundialstrukturen, vielmehr wird dem morphologischen Gegensatz zwischen Partizip und Gerund (in Interdependenz mit wortstellung und Isolierung) auch bei zuordnung zum Prädikatskomplex funktionale Relevanz zugeschrieben: allerdings bleibt die Definition und Konstituierung dieser funktionalen Unterschiede undeutlich ebenso wie der Begriff des Korrelationsmerkmals der untergeordneten Prädikativität selbst, so daß die Frage nach der Realisierung der Korrelation motiviert erscheint mit dem ziel einer empirischen Eingrenzung der untergeordnet prädikativen Funktion. Nicht von vornherein kann aus der morphologischen Form des merkmallosen Gliedes der Korrelation auf Prädikativität/Nichtprädikativität geschlossen werden, und die Entscheidung über Prädikativität/Nichtprädikativität gibt noch nicht in jedem Falle Auskunft über den Satzgliedstatus der fraglichen Form.

3.3.3. Umwertung der aksl. Korrelation

Die Korrelation im Russischen mit dem merkmalhaft untergeordnet prädikativen Gerund ist gegenüber dem Aksl. als das Ergebnis einer Umwertung der dort bestehenden Korrelationsbeziehung 
zwischen merkmalhaft nichtprädikativer Langform des Partizips und merkmalloser Kurzform $z u$ betrachten. Vgl. hierzu Ruzicka 62, Korrelationswandel, S. $680 \mathrm{f}::^{44}$

"Der Wandel dieser Korrelation vollzieht sich im Altrussischen durch ihre Umkehrung insofern, als sich das Merkmal der Nichtprädikativität zu positivem Merkmal der Prädikativität wendet, und dieses Merkmal vom bisher merkmallosen nominalen Partizip aufgenommen wird, während das bisher merkmalhaltige pronominale Partizip merkmallos wird. Die Gestalt des jetzt merkmalhaltigen Glieds wandelt sich durch Verlust der Kongruenz (Gerundium)."

Vgl. ders. 62, zur Rolle, S. 188:

"Das Kurzpartizip verliert seine Fähigkeit zur Kongruenz. Damit war die Prädikativität völlig durchgedrungen und zum positiven Merkmal erhoben... Die Funktion des zentralen Prädikats eines Nominalsatzes war an Kongruenz des Partizips gebunden. Der Verlust dieser Funktion, der im Altslawischen durch die Vorbildwirkung des Griechischen noch beschleunigt wurde, ließ die Funktion des peripheren Prädikats als die einzige prädikative zurück, von der analytischen form abgeschen (be ukp). In der peripheren Prädikation aber ist das Partizip nicht notwendig mit Kongruenz verbunden, vor allem dann nicht, wenn es auf der gleichen syntaktischen "Linie" wie das zentrale Prädikat lag, also mit ihm das Subjekt teilt. Das war bei den nominativischen Konstruktionen der Fall, und diese stehen mit ihrer struktur im Gegensatz zu allen anderen Konstruktionen: nur nominativische Formen des Prädikats sind $z u$ Gerundien erstarrt..."

Neu im Russischen ist also insbesondere der Ausfall der Kurzform im Rahmen des subjektskomplexes einerseits und die sekundärprädikative Funktion des Partizips (in der Langform) im Rahmen des Prädikatskomplexes andererseits.

Für das Aksl. gilt die Beziehung der asymmetrischen Korrelation zwischen Lang- und Kurzform des Partizips in der Weise, daß die Langform merkmalhaft nichtprädikativ fungiert und die merkmallose Kurzform untergeordnet prädikativ oder nichtprädikativ (attributiv), wobei untergeordnet prädikative Funktion bei wei-

44 Zur historischen Entwicklung der Korrelation: Rúzicka, SS, S. 22ff; vgl. z.B. auch Rúži ̌̌ka 59, Struktur, S. 544; Georgieva $68,5.98 \mathrm{ff}, 101 \mathrm{ff}$ und die dort angefuhrte literatur; M.. S. Iff; aur Entwicklung der Korrelation im Zusamenhang mit Aspekt- und Tempusbedeutung Barnet 65 
tem uberwiegt. ${ }^{45}$ Mit Ambiguität der Form hinsichtlich attributiver : (untergeordnet) prädikativer Funktion ist $z$ u rechnen, nicht in jedem Fall ist eine eindeutige Entscheidung möglich. 46

Die Funktion der Langform besteht grundsätzlich darin, die Interpretation als untergeordnet prädikativ auszuschließen, insbesondere, wenn bei potentiell ambiger Kurzform prädikative Interpretation naheläge. ${ }^{47}$ Von der Nichtprädikativität kann nicht direkt auf den Satzgliedstatus der Langform geschlossen werden, sie kann auftreten als Attribut ${ }^{48}$. subjekt ${ }^{49}$ oder Prädikatsnomen ${ }^{50}$. In jedem Falle läßt sich die Langform innerhalb der Nominalphrase hinreichend beschreiben, direkter Bezug zur Prädikatshandlung, insbesondere aspektual-temporaler Art. fehlt. "Ein temporaler Nebensatz ist für ein Vollpartizip in keinem Fall eine angemessene Ubersetzung." 51

Demgegenuber ist die untergeordnet prädikative Funktion der Kurzform wesentlich $z u$ beschreiben durch die Beziehung 52 der untergeordnet prädikativen Struktur zur satzschließenden Prädikation. Vgl. Rúžićka, SS, S. 160:

"Der hierarchisch abgestuften, peripheren Prädikation, die das Partizip innerhalb eines einfachen Satzes darstellt und erlaubt, entspricht auf der semantischen Ebene die Bezeichnung einer Handlung (des Partizips), die $\mathrm{zu}$ einer anderen (meist Handlung des finiten Verbs) in ein bestimmtes Verhältnis gesetzt ist. Darin besteht der Unterschied zur mehrfachen Prädikation gleichgeordneter oder asyndetisch angereihter finiter verbformen. Nur

45 Zur Korrelation im Aksl. vgl. Veterka 61, S. 37ff, 141, 163ff; Rủzicka, SS, S. 12ff, 170, 273ff, 346ff

$46 \mathrm{Vgl}$. Rúźicka, SS, S. 12, 68ff, 232ff, 354ff, 358; M., S. 60 Anm. 122

47 Rủžička 62, Korr., S. 679ff; ders.. SS, S. $363 f f$

48 ders., SS, S. $323 \mathrm{ff}, 327 \mathrm{ff}$

49 a.a.o. S. 274ff, 289ff, 292ff; vgl. ders. 62. Zur Rolle. S. $187 \mathrm{ff}$

50 ders.. SS, S. 280, 311: vgl. tb estb 1 jubei/ts estr $1 j u b 9$ ( $t b 1$ jubitb)

51 a.a.o., s. 282

52 a.a.o. S. $352 \mathrm{ff}, 363$ 
soviel kann als invariable Gesamtbedeutung eines peripheren Prädikats angegeben werden."

Während isolierte untergeordnet prädikative russische Gerundien insofern in den Satz hypotaktisch eingeordnet sind, als ihr Bezug auf das subjekt vermittelt ist über das Prädikat, besteht im Aksl. insbesondere zwischen Kurzpartizip am Satzanfang und dem Subjekt (Distribution: $\mathrm{P}_{k}+\mathrm{N}+\mathrm{V}$ ) eine unmittelbare abgestuft prädikative Beziehung, die der durch das satzschlieBende Prädikat etablierten nicht untergeordnet ist, aber zur Konstituierung des Satzes auf diese angewiesen und nur in Verbindung mit dieser möglich ist. 53

Der syntaktischen Korrelation hinsichtlich der Verteilung der Merkmalhaftigkeit parallel, vom system her hierarchisch untergeordnet und in ihrer Geltung eingeschränkt ist die semantische Korrelation mit dem Merkmal der Determiniertheit (Individualisierung/Generalisierung) der Langform. 54

U.E. lassen sich die ausführlichen und sehr subtilen Uberlegungen zum Verhältnis der syntaktischen zur semantischen Bestimung der Korrelation bei Razicka vorläufig so zusammenfassen, daß die merkmalhaft nichtprädikative Langform des aksl. Partizips restriktiv und nichtrestriktiv aufteten kann und in jeden Fall im Rahmen der Nominalphrase hinreichend $z$ u beschreiben ist, während die Kurzform (meist) untergeordnet prädikativ fungiert, d.h. in Beziehung steht zum satzschließenden Prädikat, oder aber nicht prädikativ, wobei den angeführten Beispielen Rủzickas und der semantischen Bestimmung der Korrelation nach Nichtprädikativität insbesondere bei restriktiver Beziehung 55 gegenüber dem Bezugsnomen gegeben ist. Bei Nichtrestriktivität tendiert die Kurzformzur untergeordneten Prädikativität; um diese auszuschließen, is t Ersetzung durch die merkmalhaft nichtprädikative Langform nötig. 56

$53 \mathrm{Vgl.} \mathrm{Razicka,} \mathrm{SS,} \mathrm{S.} 21 \mathrm{ff}$

$54 \mathrm{Vgl}$. hierzu auch Holk 63; Borodit 63; Tolstoj 57: Razicka, ss, s. 274ff, $327 f f, 347$

55 a.a.O. S. 68ff, 232-237, 354, 358

56 a.a.o. S. 328: "Bei den Partizipien ist diese semantische... 
Im Russischen tritt das Partizip (in der Langform) restriktiv und nichtrestriktiv auf; Nichtrestriktivität ist Bedingung für untergeordnete Prädikativität und gegebenenfalls Ersetzung durch Gerund.

An formalen Kriterien stehen zur Kennzeichnung von Funktionstypen im Aksl. neben dem morphologischen Gegensatz zwischen Lang- und Kurzform die wortstellung zur Verfügung, nicht aber die Isolierung. Mit ambigen Wortstellungstypen ist $z$ u rechnen. Insbesondere gilt die Möglichkeit der Ambiguität für Zwischenstellung $\left(N+P_{k}+V\right)$, während $P_{k}+N+V$ eindeutig ist bei maximal ausgeprägter untergeordneter Prädikativität.

In dem Material aus "Monumenta Serbica", das unserer Magisterarbeit zugrundelag, ergab sich grundsätzlich ein ähnliches Bild wie für das aksl. Material bei Rúzicka, ss.

Die Isolierung steht nicht als konsequent verwendetes Kriterium zur Verfügung: nicht immer kann sicher zwischen Lang- und Kurzform unterschieden werden. 57 Die syntaktische struktur dieser Urkundentexte ist z.T. so kompliziert und z.T. inkonsequent, daß die Klassifizierung nach Typen der Distribution Vereinfachungen gelegentlich unumgänglich macht, die im einzelnen am Material begründet wurden.

Die Tendenz des Gebrauchs von Lang- und Kurzformen stimmt im Ganzen überein mit den von Rúzicka formulierten Gesetzmäßigkeiten,d.h. die Kurzform tritt untergeordnet prädikativ und nichtprädikativ auf, für die Langform allerdings ist, neben der bei weitem überwiegenden nichtprädikativen Funktion, sowohl auf der Linie der Referenzidentität wie insbesondere in den nichtnominativischen Kasus anders als im Aksl. auch prädikativer Gebrauch festzustellen, so daB die konsequente Formulierung einer Korrelation mit dem Merkmal der (Nicht-)Prädi-

56 ... Opposition dadurch stark entkräftet, daß die Kurzform zur Prädikativitat tendiert, so daß die vollform auch dann zur Sicherung der Nichtprädikativität Anwendung finden muB oder kann, wenn sie über das angegebene semantische Merkmal nicht verfügt."

$57 \mathrm{Vgl}$. M., S. 60 und die in Anm. 117, 118 a.a.O. angef. Lit.. insbesondere Svane 58, S. 97 
kativität nicht möglich ist. 58

Immerhin ergibt sich auf der Linie der Referenzidentität auch hier, wie im Aksl., die Ambiguität der zwischenstellung für die Kurzform in $(N+) P_{k}+V^{59}$ und die maximale Distinktivität der Anfangsstellung, d.h.: in $P_{k}+N+V$ liegt ausschlieBlich untergeordnet prädikativer Gebrauch vor, in $\mathrm{P}_{1}+\mathrm{N}+\mathrm{V}$ ausschlieBlich nichtprädikativer (attributiver)60. Einige wenige problematische Fälle könnten angenommen werden bei $\mathrm{Zwischenstellung}$ der Langform und Endstellung der Lang- und Kurzform. 61

58 Zur prädikativen Verwendung der Langform vgl. 2.B. M., S. 55ff: zur (unproblematischen) nichtprädikativen Verwendung der Langform vgl. a.a.O.S. 59ff, $62 f f$

59 M. . S. 30ff, $61 \mathrm{ff}$

60 M., S. 36ff, $60 f f$

61 M., S. 20ff, 58, $60 f f$ 
4.

Zum Begriff der Prädikation

Für die Untersuchung von Partizipial- und Gerundialstrukturen als Formen der untergeordneten Prädikation, deren Funktion üblicherweise expliziert wird durch Paraphrasierung anhand von (Neben-) sätzen und/oder Ableitung von "tiefenstrukturellen" Konstituentensätzen, die in den Matrixsatz eingebettet oder ihm adjungiert werden, wäre Voraussetzung die klärung des Begriffes der satzkonstituierenden Funktion der Prädikation, die Antwort auf die klassische Frage "Was ist ein Satz?" . Eine allseits befriedigende Antwort auf diese Frage steht im Rahmen der Linguistik bis heute aus ${ }^{1}$ und soll hier nicht versucht werden.

4.1. Ebene des Satzes

Ein Seitenblick auf die Forschungsgeschichte ${ }^{2}$ zeigt, daß wesentlich die Frage nach dem Begriff des Satzes Anlaß war, Ebenen des sprachlichen systems zu unterscheiden. Versuchte man traditionellerweise, den Satz auf einer dieser Ebenen zu definieren, ihn hinsichtlich seiner konstituierung auf die jeweilige Ebene der Definition zu reduzieren (insbesondere: logisch, psychologisch, formal-grammatisch), so verzichtet die nevere Linguistik auf die Definition selbst bzw. setzt sie voraus und beschreibt den Satz als sprachliches Gebilde durch Unterscheidung und hierarchische zuordnung von Funktionsebenen, ${ }^{3}$ um ihn

1 Vgl. Petöfi 71, S. 248ff; Weiss 77, S. 10

2 Vgl. 2.B. Panzer 75, S. 164ff; Arens I 74, S. 394ff; Mrázek 58; Svetlik 70, S. 50ff; Jelitte 73, S. $189 \mathrm{ff;}$ Starikova 74, S. 34ff; Panfilov 71, S. 166ff; Jäger 68, S. 15ff; Rudnev 68, S. 5ff, 34ff; Schrenk 68, S. 48ff, S. 103ff; Steblin-Kamenskij 56; Krjučkov-Maksimov 69, S. 47ff

$3 \mathrm{Vgl}$. Panzer 75, S. $166 \mathrm{ff}$; AG 70, S. $541 \mathrm{ff} ;$ vgl. auch (mit ausführlichen Literaturangaben) Banaru 75, S. 4: "Mnogotislennye popytki bolee polno predstavit' predloźenie, raskryt' bolee gluboko suśćnost' i mechanizm ego Eunkcionirovanija privodjat neizbeźno $k$ ego urovnemu (ili aspektnomu) analizu... 
auf diese Weise abzugrenzen gegenüber außersprachlichen Gegebenheiten und sprachlichen Strukturen, die als Nicht-Satz identifiziert werden. Die explizite Trennung der Funktionsebenen ist bis heute, wie sich gerade bei der Erörterung von Problemen der untergeordneten Prädikation zeigt, ein noch nicht abschließend erfültes Postulat.

\subsubsection{Morphologische Bestimung}

Soweit die "komplexe syntaktische Funktion der Prädikation"4 definiert wird nach den sogenannten " prädikativen Kategorien" , betrifft dies zunächst einmal formale morphologische Merkmale des finiten Verbums in der Funktion des satzschließenden Prädikats. Die Satzstruktur, die dieses finitverbale Prädikat enthält, ist dabei vorausgesetzt. Zugrunde llegt die Tatsache, daß im Russischen, wie wohl uberhaupt in den indogermanischen Sprachen, das finite Verbum merkmalhaft die Funktion des Satzgliedes "Prädikat" erfullt und flektiert nach insbesondere Person, Tempus, z.T. nach Modus und anderen morphologischen Kategorien. 5

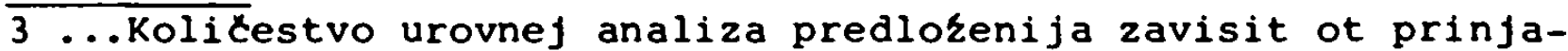
toj pri issledovanii predlozenija koncepcii i var'iruet ot avtora k avtoru.": vgl. ZfSl 75 passim, insb. die Beiträge von Danes u.a.. S. $613 f f$ ("Projekt einer theoretischen Konzeption der tschechischen Grammatik"); Koschmieder 65

$4 \mathrm{Vgl}$. 2.B. Bi rnbaum 67, Inf., S. 271: "The complex syntactic function of predication can be defined in at least two ways: either intrinsically, in terms of its constituents; or pragmatically, in terms of sentence-structure, $i . e$. in terms of those grammatically correct word strings to which predication is assigned as the basis function. Thus, predication can be said to represent the sum of its functional -obligatory as well as optional-components (the so-called predicative categories) and, on the other hand, to be the fundamental criterion whereby well-formed word strings which are identifiable as sentences of a given language can be intuitively distinguished from other equally well-formed strings which are not sentences of that language but can, say, serve as headings or title."

5 a.a.O. S. 273; Panzer 75, S. 85, 92, 110, $140 \mathrm{ff}, 163$ 
Der morphologische, einzelsprachliche und im Hinblick auf die Konstituierung des Satzes grundsätzlich fakultative Charakter dieser "prädikativen Kategorien" ist $2 u$ betonen. 6 In der Literatur, so U.E. auch noch in der AG 70, wird dabei im einzelnen die Gefahr deutlich, nicht exakt zu trennen zwischen formalen und semantischen Merkmalen einerseits und andererseits Merkmalen, die das Prädikat und solchen, die das Satzganze betreffen.

Das nach morphologischen Kriterien gekennzeichnete russische finite Verbum ist auf seine syntaktische Rolle als Prädikat im Satz festgelegt und als solches identifizierbar. Diese einzelsprachliche Tatsache darf nicht für alle Sprachen vorausgesetzt werden ${ }^{7}$ und es gibt ja auch im Russischen noch andere Formen der satzschließenden Prädikation. Nur durch das finite Verbum ist die syntaktische Prädikation als solche bezeichnet, in allen anderen Fällen nur ausgedrückt bei potentieller Ambiguität, deren Interpretation erfolgt durch von der fraglichen Form verschiedene Gegebenheiten, durch Rückgriff auf von der morphologischen Funktionsebene $z u$ trennenden Ebenen und verschiedeneArten von Kontext. Vgl. 2.B. das Gerund (insbesondere in Dialekten) in der Funktion des Prädikats: 8

vy vot vljubivsis', a cem eto koncitsja?

(Gil cenok 73, S.27)

Wenn die syntaktische Funktion von nicht "idealtypischen" Formen der satzschließenden Pradikation beschrieben wird anhand ihrer Ersetzbarkeit in syntaktischen Mustern oder Modellen durch die finitverbale Prädikation, setzt dies voraus, daß die

6 Vgl. Panzer 75, S. 167; Grabe 66, S. 72: Kubik. 66, S. 86: Starikova 74, S. $34 \mathrm{ff}$; vgl. hierzu auch die Kritik an der AG 60 bei Raspopov 7n, S. 22, 82ff, 96

$7 \mathrm{Vgl}$. Koschmieder 69, Prëdikator, S. 70: "... in einer ganzen Anzahl natürlicher Sprachen eine äußere grammatische Kennzeichnung des Prädikators einfach gar nicht existiert": Schrenk 68, S. 103

8 Jurcenko 73: Jakobson 32, S. 82: Gilcenok 73, S. 24ff, 27 : Rudnev 68, S. 36: Kuz'mina/Nemcenko 71: Kałala 71, S. 42 mit Hinweis auf Pisarkova 
nicht idealtypischen Formen in ihrer (u.a. möglichen) Funktion der satzschließenden Prädikation identifizierbar sind. Dazu bedarf es einer von den äquivalenten Formen verschiedenen vergleichsgrundlage, nämlich der Ebene des Satzes, auf der die Identifikation der Funktion und Definition der Synonymie erfolgt: eine rein formale Definition des Satzes ist nicht möglich ${ }^{9}$, den Spezialfall der finitverbalen Prädikation ausgenomen.

\subsubsection{Syntaktische Bestimmung}

Die Identifizierung des satzschlieBenden Prädikats bei nichteindeutiger morphologischer Form setzt die (intuitive) Entscheidung darüber voraus, daß einer sprachlichen Struktur, z.B. einer Folge von Wörtern, Satzcharakter zukommt; die Satzstruktur als Ganzes ist Voraussetzung dafür, daß einer Komponente die Funktion des satzschließenden Prädikats zugewiesen werden kann.

Die syntaktische "Definition" des Satzes erfolgt entweder durch Aufzählung möglicher Formen des Satzgliedes Prädikat und/oder durch konfigurationelle Darstellung in der Form $S \rightarrow N P+V P$ (bzw. $S \rightarrow$ Subjphr/Prädphr o.ä.), d.h. durch Nennung und zuordnung von als relevant angesehen Satzkonstituenten und ihre weitere Expansion. Dies ist keine Definition im strengen Sinne, vielmehr wird der Satz selbst hierbei axiomatisch vorausgesetzt $^{10}$ und dargestellt anhand eines Konstituentenstrukturmodells in deutlicher verwandtschaft zur logischen Tradition und

9 Vgl. Z.B. Schrenk 68, S. 102, 138

$10 \mathrm{Vgl}$. 2.B. Rohrer 71, S. 144: "Die Regel $\mathrm{S} \rightarrow \mathrm{NP}+\mathrm{VP}$ dürfte in der TG theoretisch nur damit begründet werden, daB sie es erlaubt, gewisse algemeine Gesetzmäßigkeiten auf einfachste Weise darzustellen. Praktisch scheint es aber so zu sein, daß diese Regel ein Relikt aus der traditionellen Grammatik darstellt..."; S. 67 69: "Chomsky sieht selbst, daß diese Definitionen zumindest voraussetzen, daß die Begriffe $S$, NP, VP, etc. selbst definiert worden sind. ... Gerade diese Definitionen fehlen aber." 
zur klassischen Satzgliedlehre. Auf der syntaktischen Ebene selbst ist der Satz nicht $z$ definieren, sondern nur $z u$ beschreiben, und insofern bleibt die satzkonstituierende Eigenschaft selbst außersyntaktisch und, wenn man die syntaktische Ebene als "tiefste" Ebene der linguistischen Beschreibung annimmt, außerlinguistisch. ${ }^{1}$ Auf der syntaktischen Ebene selbst ist demnach nicht in jedem Falle zu unterscheiden $z w i-$ schen grammatisch wohlgeformten wortketten, die in der Kommunikation als Sätze fungieren, und solchen, die Nicht-Sätze sind. Der Begriff des Satzes ist dem der syntaktischen Prädikation vorgeordnet, Prädikativität als satzkonstituierende Eigenschaft ist nicht Inhalt, sondern Voraussetzung für die syntaktische Untersuchung des Satzes, seine Repräsentierung anhand des oben angefühten Modells und die Identifizierung des Satzgliedes "Prädikat".

Grundsätzlich ist mit Ambiguität zu rechnen; vgl. Paula pulchra / dom otca

4.1.3. Kommunikative Bestimmung

Die Entscheidung, ob hier Sätze oder Nicht-Sätze vorliegen (in der Terminologie von F. Schmidt: aktuale oder potentielle Prädikation), hängt davon $a b$, welche kommunikative Funktion diese sprachlichen Formen haben, ob sie geäußert werden zum Zweck der Nennung oder der Mitteilung. ${ }^{12}$ Nur im zweiten Fall liegt durch die kommunikative Intention des Sprechers konsti-

$11 \mathrm{Vgl}$. Schrenk 68, S. 49,83

$12 \mathrm{Vgl}$. Jäger $68, \mathrm{~S} .20$; AG 70, S. 541; Raspopov 70, S. 22ff, 84ff; vgl. auch Panzer 75, S. 70ff mit Hinweis auf de Groot, und $S$. 71: "Natürlich haben auch die Wörter semasiologische Funktion wie die Morpheme; ihr Specificum ist aber, daß sie -anders als die Morpheme- in Grenzfällen auch kommunikative, d.h. Satzfunktion haben können: vgl. Einwortsätze wie zima./Pozar!/Ladno./Možno?; vgl. auch a.a.0. S. 166 
tuierte Prädikativität vor und kann das Satzstrukturmodell Anwendung finden. Die Entscheidung hieriber geschieht nach Bedingungen, die von der syntaktischen Ebene verschieden sind; im Falle der Nichteindeutigkeit der sprachlichen Form entscheidet die kommunikative Intention des Sprechers bzw. Hörers iber die Prädikativität der fraglichen struktur und $d . h$. auch über die Möglichkeit, das Ausgangssymbol "S" für die Darstellung in der Konstituentenstruktur anzusetzen. Wenn grundsätzlich mit der Nichteindeutigkeit der oberflächensyntaktischen Form $z u$ rechnen ist, hat die Satzdefinition zurückzugreifen auf eine von der syntaktischen verschiedene semantische Ebene und sie mus das Satzganze betreffen,nicht unter Voraussetzung des Satzbegriffs eine seiner Konstituenten.

Der Vorschlag, zu unterscheiden zwischen der syntaktischen Funktion des Prädikats, geleistet durch das Satzglied Prädikat, und der satzkonstituierenden, durch den sprecher in der Kommunikation intendierten Prädikativität, als einer Kategorie der kommunikativ-semantischen Funktionsebene, entspricht der Unterscheidung von syntaktischer und logisch-grammatischer Ebene bei Panfilov ${ }^{13}$ oder der Unterscheidung Raspopovs ${ }^{14}$ zwischen konstruktiv-syntaktischer und kommunikativ-syntaktischer Ebene. Dies ist kein Einwand gegen eine syntaktische Darstellung nach den Konstituenten (Hauptsatzgliedern) Subjekt und Prädikat oder gegen eine logische Darstellung nach Argument und Funktion, sondern eine Ergänzung. Oberflächensyntaktisch ambige Strukturen können je nach angenommener kommunikativer Funktion als Satz oder als Nicht-Satz interpretiert werden. Elementare Prädikationen einer logischen Repräsentation können sprachlich formuliert werden als Satz oder als Nicht-Satz, je nach kommunikati-

$13 \mathrm{Vgl}$. Panfilov 68, S. 54; zur Unterscheidung von "skazuemost'" als Eigenschaft des syntaktischen Prädikats und "predikativnost" als Eigenschaft des Satzganzen vgl. ders. 71, S. $170 \mathrm{ff}$ : ähnlich Banaru 75, S. 25ff, $47 \mathrm{ff}$

$14 \mathrm{Vgl}$. Raspopov 70, S. 29ff; Krylova 70; Halliday in Danes 74 (ed.). S. 44; Svetlik 70, S. 13; Gajsina 71, S. 36ff 
ver Funktion. 15

Die Wahl zwischen potentiell äquivalenten sprachlichen Formen und die Interpretation ambiger strukturen obliegt der kommikativen Intention. Prädikativität als notwendige Eigenschaft des sprachlichen Satzes auf der kommunikativ-semantischen Ebene ist $\mathrm{zu}$ unterscheiden von der logischen und der syntaktischen Prädikation. Wir umschreiben mit (Nicht-) Prädikativität den Unterschied zwischen Nennung und Mitteilung (Darstellung) eines Merkmals im weitesten Sinne in der Kommunikation; dieser Gegensatz manifestiert sich in der "aktualen Gliederung". 16 Vgl. Raspopov 70, S. 28:

"V samom dele, esli predlozenie est' kommunikativnaja edinica, to éto oznacaet, cto ono s neobchodimost'ju dolzno vyrazat' necto soobscaemoe. No kak raz soob'scitel'nyj charakter vyskazuemogo $v$ nem soderzanija i za$k l j u c a e t s j a v$ tom, cto ono celenapravleno, tto ono organizuetsja $v$ sootvetsvii s opredelennym kommunikativnym zadaniem i imenno éto sootvetsvie, polucaja vyraženie. $v$ castnosti, $v$ aktual'nom c̀lenenii predloženija sozdaet opecatlenie otnositel'noj polnoty i zakon Cennosti vyskazannoj mysli-pri elementarnom opredelenii predloženija.

Sleduet podćerknut', cto ukazannoe sootvetsvie vyrazaet$s j a$ imenno $v$ aktual'nom clenenii predloženija, a ne $v$ svjazi, naprimer grammati ceskogo podlezascego i grammaticeskogo skazuemogo, kak éto utverżdajut po tradicii vse nasi ucebnye posobija."

Vgl. auch a.a.0. S. 99, Anm. 1:

"...cto aktual'noe clenenie est' neobchodimoe i neotemlemoe svojstvo predloženija $i$ cto ono neposredstvenno svjazanno s konstituirujusciim predlozenie aktom predikacii."

15 Zur Unterscheidung zwischen Proposition und Außerung vgl. 2.B. Grepl 75, S. 631; Danes u.a. 75, S. 613ff; Cernachovskaja 76, S. 249ff; zum Aufbau der Satzbedeutung allgemein vgl. 2.B. Svedova 73; zur organisierenden Relgvanz der aktualen Gliederung für die Satzbedeutung vgl. Svedova 73. S. 48C, 482; Sgall u.a. 73, S. 27

16 vgl. z.B. Petöfi 71, S. 213ff, 248ff, 253ff; vgl. auch Meyer 75, S. 16: "Ich werde zur Kennzeichnung von FSP- (= funktionelle Satzperspektive - Ch.H.) Phänomenen bisweilen eine niitzliche Unterscheidung Slottys $(1936,138)$ wieder aufgreifen: die zwischen "nennen" und "darstellen". Ich werde ein Element "genannt" nennen, wenn es ganz dem Thema oder dem Rhema eines Satzes zugeordnet werden kann, ein in Thema und Rhema gegliedertes Element werde ich "dargestellt" nennen" 
Auch wenn wir uns auf den Bereich des Satzes beschränken die Bedingungen der kommunikativ-semantischen struktur des Satzes verweisen uber dessen Grenzen hinaus in den des kontextuellen Zusammenhangs im weitesten Sinne; hierin liegt einer der maßgeblichen Gründe für die fortbestehenden Schwierigkeithe einer theoretisch befriedigenden und methodisch "brauchbaren" Integration der Ebene der kommunikativen Bedeutung in ein linguistisches Modell.

Die strukturelle Relevanz der Ebene der in der Kommunikation konstituierten Bedeutung in Zusammenhang mit der Unterscheidung von predikativnost' und skazuemost' hat Panfilov fuir das Nivchische ${ }^{17}$ gezeigt, wo die Divergenz zwischen logisch-grammatischer und syntaktisch-grammatischer Gliederung Folgen hat für die syntaktische struktur selbst. Z.B. Dahl 18 verweist auf die Möglichkeit der morphologischen Signalisierung der aktualen Gliederung im Japanischen. Die Relevanz der Wortstellung für die aktuale Gliederung des Russischen haben u.a. Schaller 66 (Diss.) und Adamec 66 dargestellt.

Wenn die durch die kommunikative Intention des Sprechers konstituierten semantischen Beziehungen in regelhafter Weise auch formal signalisiert werden können, besteht -bei allen noch nicht gelösten theoretischen Problemen-kein prinzipieller

$17 \mathrm{Vgl}$. Panfilov 71, S. 173ff: "Principal'noe različie meždu predikativnost'ju i skazuemost'ju osobenno otčetlivo projavljaetsja $v$ tech slułajach, kogda nesovpadenie logičeskogo predikata so skazuemym pri ego vyraženii drugim ćlenom predlozenija privodit $k$ kacestvennoj formy skazuemogo, ne vyrazajuścego logiceskij predikat, i izmeneniju samich sintaksiceskich svjazej v sostave predloženija."

$18 \mathrm{Vgl}$. Dahl in Dahl 74 (ed.), S. 2; der englische Satz John is running ist demnach in das Japanische zu übersetzen a) John wa hasitt eiru= russ.: Ivan bezit b) John ga hasit iru = russ.: Bezit Ivan, die jap. Partikel wa kennzeichnet dabei das Thema (topic"); vgl. Sgall u.a. 73, S. 171; Dezsö-Szépe in Dahl 74 (ed.), S. 65ff zur strukturellen Relevanz der aktualen Gliederung u.a. im Ungarischen, Arabischen, Swahili; zum Französischen vgl. Banaru 75, S. 26ff; zum Tagalog, Yukaghir vgl. Novák in Danes 74 (ed.). S. $177 \mathrm{ff}$ 
Grund, Probleme der kommunikativ-semantischen Funktionsebene, d.h. insbesondere die durch die Mitteilungsintention des Sprechers konstituierte Prädikativität als notwendiges Merkmal des Satzes -sowie die untergeordnete Prädikativität als relevantes Merkmal von z.B. isolierten Partizipial- und Gerundialstrukturen- in den Bereich der Performanz zu verweisen und als außerlinguistisch von der Untersuchung auszuschließen, ist eben hier doch der Bereich der sprachlichen Bedeutung betroffen in Relation $z u$ formalen sprachlichen strukturen, also der eigentliche Gegenstand linguistischen Interesses, während (polemisch vereinfacht) eher vom außerlinguistischen Charakter der in sich heterogenen und nicht definierten Satzglieder gesprochen werden könnte mit ihrer deutlichen Affinität zur Struktur des logischen Urteils.

4.2. Kondensierte Strukturen

Raspop ov 70, S. 85 :

"Daże nużno skazat', cto ponjatie predikativnosti $v$ nasej lingvisticeskoj nauke javljaetsja nastol'ko tumannym $i$ protivorecivym, cto pol'zovat'sja im bez ogovorok praktićeski voobsonevozmoźno."

Die begrifflichen Schwierigkeiten, die sich stellen im Zusammenhang mit dem Satz und der satzschließenden Prädikation, treten verstärkt auf in Zusammenhang mit der Umschreibung der untergeordnet prädikativen Funktion z.B. von isolierten Partizipial- und Gerundialstrukturen. Die Trennung und Zuordnung von Funktionsebenen, wie sie im Bereich des Satzes formuliert oder doch wenigstens gefordert wird, ist für untergeordnet prädikative strukturen noch nicht mit gleicher Konsequenz diskutiert. In diesem zusammenhang werden die verschiedensten Gesichtspunkte angeführt, die ja alle auch eine Rolle spielen, nur, Status und Relevanz dieser verschiedenen Aspekte der Beschreibung z.B. von Partizipial- und Gerundialstrukturen werden nicht recht deutlich; noch mehr als im Bereich des Satzes besteht hier die Versuchung, ad hoc Funktionsebenen zu vermi- 
schen. Damit ist auch der empirische zugang zu einer Unterscheidung von Funktionstypen erschwert.

Auf den Begriff des Satzes, der für die syntaktische Untersuchung und Darstellung axiomatisch vorausgesetzt ist, muß nun, anläßlich der untergeordnet prädikativen strukturen, innerhalb des Satzes nochmals zurückgegriffen werden; dies mag es nahelegen, die Tatsache der untergeordneten Prädikativität zu reduzieren auf von ihr verschiedene formale und semantische Merkmale anderer Funktionsebenen. Die Kritik hierzu mübte analog geführt werden zur Diskussion um die Frage "Was ist ein Satz?" und es darf wohl die These gewagt werden, daß die Spezifik und die spezifischen Schwächen der jeweils zugrundegelegten linguistischen Konzeption gerade anläßlich der Probleme der untergeordneten Prädikation sichtbar werden und Anlaß gaben $z u$ Kritik und Weiterentwicklung dieser Konzeptionen. 19 Die Formulierung der "klassischen" TG auf der Grundlage der Theorie der rekursiven Funktionen wie der generativen Semantik und die Anwendung der intensionalen Logik bei Bartsch 72 sind wesentlich verknüpt mit Fragen der untergeordneten Prädikation: gerade anläßlich dieser Fragen wird das Problem der sprachlichen Bedeutung und der Notwendigkeit der Uberschreitung der Satzdomäne diskutiert. 21

Als Beispiel für die heterogene vielfalt formaler und funktionaler Gesichtspunkte, die angefuhrt werden zur Umschreibung der untergeordneten Prädikativität von (hier: deutschen) Parti-

$19 \mathrm{Vgl.2.B.}$ die Forschungsübersicht bei Rudnev 59, S. 7ff ; Kaðala 71, S. 10-53

$20 \mathrm{Vgl}$. Rohrer 71, S. 17ff, 22 mit Hinweis auf Post; vgl. auch die Darstellung von Adverbialstrukturen anhand von Prädikationen uiber Sätzen bei Lakoff und die Diskussion hierzu und die bei Bartsch/V. 72, Bartsch 72, Immler 74, Rohrer 71 angefuhrte und diskutierte Literatur: Prager Autorengruppe 75; Sgall u.a. 73; Weiss 77, S. $13 \mathrm{ff}$

21 vgl. z.B. Petöfi 71, S. $248 f f$ sowie die wesentlich in zusammenhang mit Fragen der untergeordneten Prädikation geführte Diskussion $\mathrm{zu}$ einem neuen Grammatikprojekt in $\mathrm{zfSl} 75$; vgl. a.a.O. die Beiträge von Hausenblas, Michnevič, Ivančev zur kontextuellen Interdependenz der Kondensation 
zipialkonstruktionen kann Bungarten 76 dienen. Die Prädikativität des Partizips ist demnach bedingt durch die "Wertigkeit" des zugrundeliegenden Verbs, 22 diese begründet den satzähnlichen Charakter des Partizips: gleichzeitig ist das Vorhandensein einer Prädikation eindeutig signalisiert durch die Isolierung ${ }^{23}$ des Partizips. Der prädizierende Charakter der adverbialen Partizipialkonstruktion bedingt ihre Fakultativität. 24 Diese Fakultativität, hier gleichzeitig begründet anhand von Fügungseigenschaften des übergeordneten Verbums. wird als Analogon zur Nichtrestriktivität des attributiven 25 untergeordnet prädikativen Partizips gesehen. Diese aber ist nicht durch Valenzeigenschaften begründet. Neben der oben angeführten wertigkeit des zugrundeliegenden Verbs, der Isolierung und Nichtrestriktivität wird als Begründung für die Prädikativität des attributiven Partizips seine semantische Beziehung zum Prädikat angeführt. Einen "ganz anderen prädikativen Charakter" 26 haben attributive Partizipialkonstruktionen, die eine lexikalisch explizite Charakterisierung des Bezugsnomens hinsichtlich Raum und zeit enthalten. Gleichzeitig wird davon gesprochen, daß die attributiven Partizipialkonstruktionen nicht in jedem Falle ihre prädikative Funktion verlieren und häufig noch einen prädikativen Zug aufweisen, 27 so daß nicht ganz klar ist, ob es auch isolierte nichtrestriktive nichtprädikative Partizipien gibt. Adverbiale Partizipialstrukturen haben in jedem Falle Bezug auf das Satzganze oder zumindest zum Prädikat. 28

All diese Gesichtspunkte können für das einzelne Beispiel zutreffen, ihr im ganzen heterogener und subjektiver charakter

22 d.h. durch den verbalen Charakter des Partizips, also eine derivative Eigenschaft

$23 \mathrm{Vgl}$. Bungarten 76, S. $133 \mathrm{ff}, 208,256$

24 a.a.o. S. 135

25 a.a.o. S. 136,152

26 a.a.o. S. 154

27 a.a.o. S. $154 \mathrm{ff}, 176$

28 a.a.O. S. 175 
ist letztlich Indiz für die bis anhin notwendig fehlende Vermittlung zwischen dem Begriff des Satzes und der untergeordneten Prädikativität.

4.2.1. Semantische Bestimmung

Nicht weiter diskutiert werden soll hier die Auffassung, die Prädikativität von Partizipial- und Gerundialstrukturen ergebe sich aus ihrer "Handlungsbedeutung": diese Bedeutung mag zwar in vielen Fällen zutreffen, sie ist aber kein Definitionsmerkmal der Prädikativität. Zugrunde liegt hier die semantische Definition der Wortart Verbum durch den behaupteten außersprachlichen Charakter der Handlung, Prozessualität u.ä., wobei die Gefahr eines Zirkelschlusses deutlich ist in dem Sinn, daß dann eben als Prozeß usw. klassifiziert wird, was in der sprachlichen Form des Verbums auftritt. 29 Wenn uberhaupt, so wird auf diesem weg (in fragwürdiger Weise) eine Wortart definiert und nicht die Funktion der Prädikativität. Gerade für Partizipial- und Gerundialstrukturen wird, nach Bedingungen der Wortstellung, des Aspektes und des Kontextes, im ubrigen ja nicht nur "Handlungsbedeutung" angeführt, sondern auch die Bedeutung eines zustandes als Resultat einer Handlung u.a. 30 Prädikativität, Verbalität und Handlungsbedeutung lassen sich nicht auf einander zurückführen. Dies gilt auf der Ebene des Satzes und analog für untergeordnet prädikative strukturen.

Die Tatsache der Bedeutung einer Handlung, eines Prozesses $u . \ddot{a}$. betrifft das außersprachlich Bezeichnete und besagt nichts über den kommunikativ relevanten Unterschied zwischen "Nennen" und "Mitteilen".

29 so z.B. bei Mulisch 75, S. 196, Vinogradov 47, S. 388ff, Ananic 72, S. 52ff, Isacenko 75, S. 206ff, 273ff, Rudnev 68, S. 155; zur Kritik an der semantischen Definition von Wortarten vgl. Panzer 75, S. 85

$30 \mathrm{Vgl}$. Rưzicka, SS, S. 84ff; Paffen II, S. 372 zu aksl. bzw. russ. nichtipf. Strukturen: vgl. auch Svetlik 70, S. $68 \mathrm{zu}$ "halbprädikativen" Gerundien in Zustandsbedeutung 


\subsubsection{Logische Bestimmung}

Durch die struktur des außersprachlich Bezeichneten bestimmt ist U.E. auch der Begriff der (impliziten) Prädikativität bei Starikova 74 und ähnlich in der generativen Semantik, ${ }^{31}$ insofern dort aufgrund vorausgesetzter ontologischer Verhältnisse komplexe sprachliche strukturen lexikalisch dekomponiert und die Strukturen dieser Dekomposition logisch-semantisch dargestellt werden.

vgl. 2.B. Starikova 74, S. 36

"Predikativnost' $v$ takom ponimanii moket byt' prisuśća ne tol'ko vyskazivaniju, kak aktualizirovannoj jazykovoj edinice, no i slovosočetaniju i otdel'nomu cllenu predlozenija, obladajuśćemu propozitivnym soderzaniem. Pri transpozicii predlozenija v zavisimuju sintaksiceskuju poziciju ono utracivaet svoju kommunikativnuju avtonomnost', no ego nazyvnaja funkcija (to est 'oboznaŁenie sobytija ili fakta) sochranjaetsja."

Vgl.a.a.o. S. 16

"Predlozenie, kak izvestno, po otnoseniju k ob-ektivnomu sobytiju javljaetsja imenem, a ob-ektivnoe sobytie po otnoseniju $k$ predlozeniju vystupaet ego denotatom. Predlozenija otlićajutsja ot slov specifikoj ich denotativnego znacenija. Tak, denotatom predlozenija v otlicie ot denotata slova (suśćestvitel 'nogo) ne mozet byt' "vesc"", konkretnyj predmet. V etom smysle nominacija sobytija moket byt' protivopostavlena nominacii predmeta. Predlozenie vyrazaet sobytie, a ne predmet."

Zwischen der grammatisch geformten oberfläche und der logischsemantischen Tiefenstruktur besteht nach Starikova ein asymmetrisches Verhältnis, das zur Folge hat, daß in der Tiefenstruktur explizite Prädikationen an der oberfläche implizit auftreten. Die Explikation erfolgt durch die Dekomposition und Rückführung auf elementare zweigliedrige Propositionen. Auf dieser Ebene wird eine Proposition nicht begrüdet durch die Intention der Mitteilung im Gegensatz zu der des Nennens, sondern gleichzeitig logisch und ontologisch durch die Bezeichnung eines Ereignisses oder einer Tatsache statt eines Gegenstandes.

31 Vgl. z.B. Frantz 74, S. 22ff; Starikova 74, S. 14ff, 20ff, $33 \mathrm{ff}, 36 \mathrm{ff}$ 
F. Schmidt unterscheidet zwischen logischer, ontologischer und sprachlicher Prädikation. Die sprachliche Prädikation wird aktual oder potentiell formuliert, logisch oder ontologisch ist dieser grammatische Unterschied irrelevant. 32 Der Unterschied zwischen aktualem und potentiellen Satz wird auf der syntaktischen Ebene erörtert, Fragen der kommunikativsemantischen Gliederung werden nur beiläufig angesprochen (subjektiver Akzent"). 33

Vgl. als Beispiel für aktualen Satz: Paul ist schön Vgl. als Beispiel für potentiellen Satz: Der schöne Paul (Attribut)

Bei der Formulierung sämtlicher in der sprachlichen struktur impliziten und expliziten Prädikationen anhand logischer Profositionen ist die spezifische untergeordnete Prädikativität von Partizipial- und Gerundialstrukturen nicht mehr darstellbar, vielmehr entspricht sprachlich aktualen Sätzen, potentiellen Sätzen 2.B. in der Form des Attributs, einer in einer komplexen lexikalischen struktur formulierten impliziten Präcikation und untergeordnet prädikativen strukturen die gleiche Form der Proposition. 34

Vgl. Weiss 77, S. 64 (in Zusammenhang mit der Definition des Partizips) :

"In allen Fällen liegt die Reduktion eines tiefenstrukturellen Satzes zu einem Syntagma vor, das keine Satzfunktion mehr erfüllt. Partizipialkonstruktionen gehen also jeweils auf einen ganz durch das Symbol "S" dominierten Teilbaum in der Tiefenstruktur zurück, das Partizip allein stellt die Weiterentwicklung des Knotens "V" in diesem Teilbaum dar. Für eine kategorielle Definition ist diese Gemeinsamkeit wiederum nicht ausreichend, da dasselbe auch fur Infinitive bzw. -konstruktionen gilt. Im Prinzip kann ja ohnehin jedes Element auf eine zugrundeliegende satzwertige Prädikation zurückgefuhrt werden, wie z.B. die in der Generativen Semantik vorgeschlagene Behandlung von Nomina... oder der Satzmodalität zeigt."

$32 \mathrm{lgl}$. F. Schmidt 70, S. 25, 45ff; ders. 61, S. 32ff; steblin-Kamenskij 56, S. $133 \mathrm{ff}$ : vgl. zu schmidt 59 roschmieder 59

$33 \mathrm{igl}$. Schmidt 70, S. 18

$34 \mathrm{igl}$. Weiss 77 , S. 82 
Es dürte schwierig sein, eine Grenze anzugeben, jenseits derer keine weitere Dekomposition und Formulierung noch einer Proposition einer nächsttieferen Stufe der Darstellung möglich ist, wenn man als Bezugspunkt den Bereich des außersprachlich Bezeichneten annimmt. In unserem Zusammenhang ist die Funktion von Partizipial- und Gerundialstrukturen in der sprachlichen Kommunikation und damit auf der Ebene der sprachlichen Bedeutung in ihrer zuordnung $z u$ oberflächensyntaktischen Strukturen von Interesse als ein Beispiel für die linguistische Relevanz der Deprädikation.

Eben dieser Unterschied düfte auch im Hintergrund stehen, wenn Sgall auf die offene Frage hinweist, welche sprachlichen Erscheinungen zur "Deprädikation" gerechnet werden sollen. 35

Eine kommunikativ indifferente logisch-semantische Form der Darstellung erfaßt nicht die spezifische Funktion der Partizipial- und Gerundialstrukturen, ihre graduell abgestufte Prädikativität, sondern gibt zunächst einmal nur darüber Auskunft, daß über das Subjekt des Satzes nicht nur das satzschlieBende Prädikat ausgesagt wird, sondern auch das in der Partizipial-bzw. Gerundialstruktur enthaltene.

\subsubsection{Syntaktische Bestimmung}

Die generative Syntax definiert den Satz nicht, sondern setzt ihn voraus (s.o.). Bei der Darstellung untergeordnet prädikativer Strukturen wird innerhalb des Satzes nochmals auf dieses Axiom zurückgegriffen als Grundlage für die Ableitung von "zweibasigen" Ausgangsstrukturen, hier mit identischem und zu tilgendem Subjekt für die Partizipial-bzw. Gerundialstrukturen.

Eine Vermittlung $z$ wischen dem vorausgesetzten und nicht definierten Begriff des Satzes und dem der untergeordneten Prä-

$35 \mathrm{Vgl}$. Sgall in 2fSl 75, S. 786: "Vieles bleibt unklar"; zur Kritik an der generativen Semantik hins. der undifferenzierten Dekomposition und Darstellung von Prädikationen über Sätze vgl. 2.B. Bartsch 72, S. $289 \mathrm{ff}$, 315ff, Bartsch/V. 72, S. $18 \mathrm{ff}$, Meyer 75, S. $37 \mathrm{ff}$, Immler 74, S. $112 \mathrm{ff}, 138 \mathrm{ff}$ 
dikativität innerhalb des satzes ist damit nicht gegeben.

die Tatsache der untergeordneten Prädikativität wird zurückgefuhrt auf die syntaktisch-tiefenstrukturelle explizite Prädikation eines Konstituentensatzes mit deutlicher Verwandtschaft zur logischen Formulierung der elementaren Proposition. 37 Das Dilemma wird darin sichtbar, daß, während eben der Begriff des Satzes vorausgesetzt wird, auch in der syntaktisch orientierten Literatur vielfache und heterogene Umschreibungen zur kommunikativ-semantischen Funktion der untergeordneten Prädikativität gegeben werden, um die Ahleitung von zugrundegelegten zweibasigen Strukturen $z u$ motivieren, die die untergeordnete Prädikativität expliziert. In diesem zusammenhang werden formale und semantische Merkmale genannt, und es ist dabei nicht immer scharf zu trennen zwischen Bedingungen und Kriterien, so auch noch z.B. bei Rúzicka 66 in zusammenhang mit dem sogenannten "sekundären Prädikat".

Angeführt werden in diesem Zusammenhang insbesondere die formalen Kriterien der Isolierung und der Wortart (Partizip, Gerund. Infinitiv als sogenannte Halbsatzkonstruktionen) ${ }^{38}$ sowie die Tatsache der Fakultativität im Hinblick auf Valenzeigenschaften des übergeordneten Verbums, der Nichtrestriktivität gegenüber dem Bezugsnomen und die Tatsache des Bezuges auf das Subjekt und Prädikat gleichzeitig, in der Tiefenstruktur dargestellt durch das identische subjekt des Konstituentensatzes.

$36 \mathrm{Vgl}$. Weiss 77, S. 64, Anm. 73: "Rückblickend... ist hier die Relativität einer Definition des Begriffes "Satz" festzuhalten, die in der TG ebenenspezifisch ausfallen muißte, da "S" in der Tiefenstruktur eine ganz andere Extension aufweist als in der Oberflächenstruktur."; vgl. auch Schrenk 68, S. $175 \mathrm{ff}, 225$

37 Zur transformationellen Explikation zweibasiger strukturen vgl. 2.B. Rủzicka 62, Korr., S. 680, ders. 66, ders. 65, verbalphr.. ders. 73, Kade 68, Schrenk 68, S. $217 f f$, Adamec II 75, S. 5ff, 27ff, 171ff, 179ff, Babby 75, Krizková 69, Synt. ch.. dies. 69, Po, dies. 67, Problemy, S. $77 \mathrm{ff}$ dies. 68 , IsaXenko 63, Transf. An., Uspensif 63, Kubik 66, Weiss 74, ders. 77, u.v.a.

$38 \mathrm{Vgl.2.B}$. Svetlik 70, S. 265ff; Kacala 71, S. 101ff 
Indirekt wird mit diesen Hilfskriterien die (untergeordnete) Prädikativität Gegenstand der Untersuchung innerhalb des Satzes, während sie für den Satz selbst als nicht zu definierende Voraussetzung angenommen wird. Rekonstruiert wird die Tatsache der untergeordneten Prädikativität anhand der Geschichte der Ableitung von einer expliziten tiefenstrukturellen Prädikation - es wurde bereits darauf verwiesen, daß ähnliche Derivationen für die verschiedensten Arten impliziter Prädikation angefuhrt werden, so daß sich die Frage stellt, wie die hinsichtlich ihrer kommunikativen Funktion graduell abgestufte untergeordnete Prädikativität der Partizipial- und Gerundialstrukturen zu spezifizieren ist, wenn sie unterschieden werden soll von der potentiellen Prädikation im Sinne von $F$. Schmidt z.B. bei attributiven strukturen.

wichtiger als eine ins einzelne gehende Kritik zur Rückführung der untergeordneten Prädikativität in der syntaktischen Sekundärliteratur auf die oben angefühten formalen und semantischen Merkmale scheint uns die Tatsache, daß eben diese formalen Merkmale nicht so sehr syntaktisch als semantisch signifikant sind und daß die semantischen Merkmale über die Grenzen des Syntagmas und den Bereich der syntaktischen Funktionsebene hinausführen. 39

Wenn, aufgrund welcher formalen und semantischen expliziten oder intuitiv erfaßten Kriterien auch immer, die Motivierung der Ableitung von einer tiefenstrukturell expliziten Prädikation als gesichert angenommen wird mit dem Ziel der Reformulierung der untergeordneten Prädikativität mit syntaktischen Mitteln, kann aus der Tatsache der untergeordneten Prädikativität und der Ableitung noch nicht unmittelbar auf die Art der Verknüpfung mit dem aufnehmenden Satz geschlossen werden.

39 Zur Uberschreitung des Syntagmas durch die relevanten semantischen Beziehungen isolierter Wendungen vgl. z. B. Michnevic 68, S. 106ff; zu nichtrestriktiven Partizipialkonstruktionen Kornilov 67: vgl. auch SCeboleva 71, S. 101ff; Valgina 72; Koljadenko 72, S. 89 
In den Darstellungen von Rúžička 66 und Weiss 77 wird diese Frage als weitgehend gelöst vorausgesetzt, d.h. Probleme der Ambiguität der Oberfläche stellen sich hier kaum, wie sie sich unvermeidlich ergeben, sobald man versucht, nach den z.B. bei Rúzicka 66 angedeuteten Kriterien Material zu klassifizieren: die paradigmatische Ersatzprobe anhand von attributivem Relativsatz bzw. adverbialem Nebensatz, wie sie insbesondere Bungarten anwendet, gibt strenggenomen nur Auskunft über die Ersetzbarkeit einer oberflächenstruktur durch eine andere, nicht aber z.B. darüber, daß ja tiefenstrukturell die Grenze zwischen Einbettung und Verknüpfung von Prädikationen fließend sein kann. ${ }^{40}$ Weiss z.B. begründet diese Unterscheidung nicht syntaktisch, sondern kommunikativ-semantisch, indem er nur für restriktive Partizipien adnominale Einbettung vorsieht, für alle nichtrestriktiven und sekundärprädikativen Strukturen aber Konnexion von Sätzen.

Im Russischen ist dann, anders als im Polnischen, auch für akt. Partizipien nicht nur mit attributiver funktion zu rechnen, sondern auch mit sekundärprädikativer.

Die Paraphrasierung von Partizipial- und Gerundialstrukturen durch Nebensätze 41 , also Formen der aktualen, aber syntaktisch untergeordneten Prädikation, besagt u.E. zunächst einmal nur etwas über die Ersetzbarkeit, nicht aber uber die untergeordnete Prädikativität der Ausgangsstrukturen. 42 Auch nichtprädikative Strukturen (vgl.: der schöne Paul : Psul, der schön ist; der pflugende Bauer : der Bauer, der pflügt) können durch finitverbale Nebensätze paraphrasiert werden. Gerade wenn man

$40 \mathrm{vgl}$. Hartung 73, S. 39ff, 50ff; Fillmore 63, S. 208ff

41 F. Schmidt 61 , S. $74 \mathrm{ff}$ ordnet Nebensätze ein zwischen aktualer und potentieller Prädikation. Dies ist u.E. zumindest mißverstandlich. Die Prädikation im Neben-Satz ist doch nicht weniger aktual als die des Haupt-Satzes, der Unterschied besteht in ihrer syntaktischen Unterordnung

42 Vgl. 2.B. auch Paffen II, S. 366 und demgegenüber Rudnev 59, S. 66 zum Nachweis der "Verbalität" bzw. "Prädikativität" mittels Ersetzung durch Verbum finitum; vgl. ders. 63, S. 170, a.a.0. S. 176; Svetlík, S. 265ff; AnaniC S. 55, 58, 60ff: Babby 75 
die untergeordnet prädikativen Partizipial- und Gerundialstrukturen ansieht als eine von mehreren möglichen Realisierungen einer vorausgesetzten Satzgliedposition, ist durch die Ersetzung innerhalb des Paradigmas nicht Auskunft gegeben über die Spezifik der zu erklärenden struktur, die untergeordnete Prädikativität, sondern über das beiden oberflächenstrukturen Gemeinsame, die angenommene Satzgliedposition.

Fuir die Definition einer Satzgliedposition ist die Ableitungsgeschichte einer von mehreren möglichen Realisierungen, auch wenn sie die Identität des Subjekts expliziert, im Grunde nicht relevant, $d . h$. in unserem zusammenhang: im Rahmen eines an vorausgesetzten Satzgliedpositionen orientierten syntaktischen Modells ist die kommunikativ konstituierte untergeordnete Prädikativität im Grunde nicht darstellbar. Soweit die Ableitungsgeschichte selbst als Explikation der untergeordneten Prädikativität herangezogen wird zur Definition einer Satzgliedposition, wie z.B. im Falle des sekundären Prädikats bei Rúżicka, ergibt sich doch der verdacht, daß die Begründung hier oberflächensyntaktischer Art ist. ${ }^{43}$ Dies ist jedoch kein Einwand gegen die funktionale Spezifik von isolierten Partizipial- und Gerundialstrukturen, sondern gegen die Möglichkeit der Darstellung in einem syntaktischen Modell. Bezeichnend für das Dilemma erscheint uns Kac̈ala 71, wo die sekundäre Prädikativitat ebenfalls herangezogen wird zur Definition einer Satzgliedposition ("doplnok"), deren semantische Abgrenzung gegenüber adverbialen Bestimmungen und gegenuber untergeordnet prädikativen, jedoch nicht als "doplnok" fungierenden Halbsatzkonstruktionen allerdings nicht recht deutlich wird. Kačala 75 sieht demgegenüber untergeordnete Prädikativität als Möglichkeit in jeder syntaktischen Position vor. 44

$43 \mathrm{Vgl}$. Panzer 75, S. 178

$44 \mathrm{Vgl}$. Kaćala 71, S. 101ff, 118ff; ders. 75, S. 798ff; ders. 69, S. 708 sieht einerseits die Möglichkeit, daß ein Nebensatz als doplnok (sekundäres Prädikat) fungiert und ordnet andererseits die sek. Prädikativität des "sek. Präd." ein zwischen der Nichtprädikativität des Attr. und der Prädikativität des Nebensatzes: zur Unterscheidung von Satzgliedpositionen und untergeordneter Prädikativität vgl. 2.B. Picugov 73 
4.2.4. Kommunikative Bestimmung

Unsere Uberlegungen zur Spezifizierung der untergeordneten Prädikativität auf der Ebene der durch die kommunikative Intention konstituierten sprachlichen Bedeutung analog zum Begriff des sprachlichen Satzes laufen parallel mit den Hinweisen von Weiss 77 zur Unterscheidung von Prädikation und Attribution hinsichtlich ihrer kommunikativen Leistung, d.h. der "Zuordnung eines Merkmals in Form einer Aussage" einerseits und andererseits einer "supponierten Zuerkennung". 45

vgl. Weiss 77 , s. 82ff:

"Ontologische Kategorien scheiden hierbei aus, der ausgedrückte Sachverhalt ist ja in beiden Fällen derselbe; es muß sich also um eine sprachinterne Differenzierung handeln. Klemensiewicz vertritt hier die traditionelle Ansicht von der Hierarchie der verschiedenen syntaktischen Abhängigkeitsverhältnisse ... Damit ist zwar über das Wesen von Attribut und Prädikation noch wenig besagt, weiter kommen aber auch moderne Darstellungen meistens nicht, mit eingeschlossen transformationelle Untersuchungen, die nicht nur um keine Definition bemüht sind, sondern den Unterschied zwischen den beiden Typen auf der untersten Beschreibungsebene gar aufheben, indem sie die Attribution auf eine Satzeinbettung und damit auf eine Prädikation zurückführen -ein Vorgehen, das ... auf den kognitiven Kern der Außerung, nicht auf ihre Bedeutung abstellt."

Auf die kommunikative Spezifik der untergeordneten Prädikativität z.B. von isolierten Partizipial- und Gerundialstrukturen verweist, mit unterschiedlicher Terminologie, die Erörterung der kommunikativ-semantischen Signifikanz der formalen Kriterien Wortstellung und Isolierung in der Literatur, so z.B. auch in der AG 70 und bei Rudnev. ${ }^{46}$ Die (untergeordnete) Prä-

$45 \mathrm{Vgl}$. Weiss 77, S. 83 in Anlehnung an Jodlowski

$46 \mathrm{Vgl}$. Rudnev 68, S. 36; Georgieva 68, S. 100; Rudnev 68 , S. $149 \mathrm{ff}$ : "Obosoblennoe opredelenie, $v$ otlicie ot neobosoblennogo, s tocki zrenija semantiki charakterizuetsja tem, cto oznacaet ne prosto priznak predmeta,a priznak, pripisyvaemyj predmetu, t.e. imejustij predikativnoe znacenie. Pod predikativnost'ju, $v$ otlitie ot rjada lingvistov, no $v$ soglasii s Potebnej .. my ponimaem vyrazenie nepolnogo vyskazyvanija..."; zur graduellen Abstufung der Prädikativität vgl. Rudnev 59, S. 56, Kornilov 64, S. 97, ders. $67, \ldots$ 
dikativität isolierter Wendungen ergibt sich aus ihrem (abgestuften) Charakter einer Mitteilung, der von der bloBen Nennung eines Merkmals (z.B. durch ein nichtisoliertes Attribut) zu unterscheiden ist.

Logisch und ontologisch ist dieser Unterschied nicht zu spezifizieren, doch hat er Konsequenzen für die stellung der Partizipial- und Gerundialstrukturen auf der Ebene der durch die kommunikative Intention des Sprechers konstituierten sprachlichen Bedeutung, die ihren Reflex findet in den formalen Kriterien der aktualen Gliederung ${ }^{47}$ und durch Testverfahren zu explizieren ist.

Für das Russische rekonstruieren im Grunde Kornilov und Kríiková die asymmetrische Korrelation mit dem merkmalhaft untergeordnet prädikativen Gerund anhand des Merkmals der Nichtrestriktivität; ${ }^{48}$ Nichtrestriktivität ist Voraussetzung für untergeordnete Prädikativität. Das Partizip kann restriktiv und nichtrestriktiv gebraucht werden, nur bei Nichtrestriktivität gegenuber dem Subjekt ist allenfalls Transposition in den Prädikatskomplex und Ersetzung durch Gerund möglich.

Weiss 77 sieht nur für nichtrestriktive Attribute und sekundärprädikative Strukturen im Rahmen des Prädikatskomplexes Ableitung von der Satzkonnexion anhand des "logischen und" in der Tiefenstruktur vor, ${ }^{49}$ restriktive Attribute werden in der Tiefenstruktur durch adnominale Einbettung repräsentiert.

46 ....S. 7, M., S. 44ff, Anm. 88, Weiss 77, S. 239, Ktizková 67, Problemy, S. 77: zur Variabilität der untergeordn. Prädikativität vgl. Rưžcka, SS, S. $57,67,69,89,348,352,366$; vgl. auch ders. 73

$47 \mathrm{Vgl}$. zur stellung von isolierten wendungen in der aktualen Gliederung Danes in Danes 74 (ed.). S. 106ff, Ceremisina 71 , S. 20, Krylova 70, S. 90, Kornilov 67, S. 15ff, Kłizková 68, dies. 69, PO: vgl, auch Rúźicka, SS, S. 359: "Es ist unumgänglich, auch von einer Graduierung der Prädikativität $2 u$ sprechen, an der die "aktuelle" Gliederung des Satzes beteiligt ist."

$48 \mathrm{Vgl}$. auch Źaźa 65; Dozorec 72; Bungarten 76, S. 135, 152: Seiler 60, S. 36ff: Ktiźková 69, PO, S. 8; dies. 68; s. auch Weiss 77, S. $87 \mathrm{ff}$ und die dort angeführte Literatur: "Danach schränken restriktive Relativsätze die Extension des der spezifizierten NP zugrundegelegten Begriffes ein, explikative nicht."

$49 \mathrm{Vgl}$. Weiss $77, \mathrm{~S}, 356 \mathrm{ff}, 367$ 
Dies hat Folgen für die stellung in der aktualen Gliederung 50 und für mögliche syntaktische Funktionen ${ }^{51}$, die im einzelnen am Material zu erörtern sind. Dem Unterschied zwischen Repräsentierung durch adnominale Einbettung und Satzkonnexion liegt der funktionale Gegensatz zwischen Referenzakt und Prädikation zugrunde: 52

Vgl. Weiss 77 , s. 239:
"Dem ... klassenlogischen Aspekt entspricht der unter- schiedliche pragmatisch-textlinguistische Effekt der beiden Typen. Die Hervorhebung eines objekts bzw. einer Klasse von Objekten aus einer umfassenden Klasse gleich- artiger objekte mithilfe der restriktiven Attribution geschieht zum Zwecke der Identifikation .... Angesichts dieser individuierend-referierenden Funktion sind re- striktive Attribute zwar Bestandteile von Sprechakten, doch die Nennung von Bezugswort + Attribut ist selber als Referenzakt $z u$ werten. Demgegenuiber können nicht- restriktive Attribute keine objekte neu etablieren: sie bieten vielmehr eine neue, als selbständige Aussage formulierte Information uber einen bereits etablierten Gegenstand und sind daher eher als Spezialfall der Prä- dikation $z u$ werten, wobei das spezifikum in der gegen- über der Hauptprädikation geringeren Wertung dieser In- formation durch den Sprecher besteht. Auf diesen "Hin- tergrundcharakter" nicht-restriktiver Attribute, den sie mit Part-II-Konstruktionen teilen, wird noch zuruckzu- kommen sein..."

Auch wenn die Integration der Ebene der in der Kommunikation konstituierten sprachlichen Bedeutung in ein linguistisches Modell noch aussteht und die kommunikativ-semantische Definition der (untergeordneten) prädikativität ein -allerdings u.E. durch gute Gründe zu motivierendes- Postulat bleibt, erscheint es als plausibel, eine provisorische empirische Eingrenzung des vage umschriebenen Unterschiedes zwischen Nennen und Mit50 Vgl. a.a.O. S. 357 ; Krylova 70, S. 86ff, 90; Lapteva 72;
Sgall in Danes 74 (ed.), S. 64

$51 \mathrm{Vgl}$. Weiss 77, S.90ff, 247, 357

$52 \mathrm{Vgl}$. a.a.O. S. $238 \mathrm{ff}, 384$, S. 312, Anm. 410 
teilen 53 dadurch $z u$ versuchen, daß nach formalen und funktionalen Kriterien der stellung der untergeordnet prädikativen Partizipial- und Gerundialstrukturen auf der Ebene der sprachlichen Bedeutung gefragt wird, d.h. nach kommunikativ konstituierten semantischen Beziehungen. Diese Beziehungen werden verstanden als Typen und Varianten der fakultativen Konnexion von hierarchisch zugeordneten Prädikationen. Diese Beziehungen organisieren bei vorausgesetztem Denotat und gegebenen lexikalischem Material für die fakultativen und ambigen Partizipialund Gerundialstrukturen funktionale Unterschiede ${ }^{54}$, die z.T. implizit auch in der syntaktischen Terminologie zugrundeliegen, so u.E. z.B. bei Rúžicka 66, ohne daß hier die Konstituierung der Funktionstypen erörtert wiirde, was den Vorwurf der Unmotiviertheit und oberflächenorientiertheit der hier syntaktisch beschriebenen Funktionstypen zur Folge hatte.

Schon bei unserer Untersuchung des Materials an Partizipialkonstruktionen aus den "Monumenta Serbica" ergab sich die Vermutung, daß die graduell abgestufte Prädikativität der Partizipien wesentlich durch die Beziehungen zwischen uber- und untergeordneter Prädikation bestimmt ist in Abhängigkeit von der Sprecherintention. Dort war versucht worden, die unterschiedlichen Beziehungen zwischen uiber- und untergeordneter Prädikation nachzuweisen anhand von im sprachlichen Material belegten Paraphrasen (siehe Lit.verz. unter Miklosich, F. (ed.)).

$53 \mathrm{Vgl}$. Rúżčka 62, Korrelationswandel, S. 685: "Die Interpretation grammatischer Erscheinungen als asymmetrischer Korrelation stuitzt sich auf semantische Merkmale. Gerade die unumgängliche Aufnahme semantischer Beschreibungen in ein grammatisches Modell muß noch als Crux der strukturellen Grammatik gelten. Die Aufnahme der asymmetrischen Korrelation und ihre Uberfuhrung in beweisbare Bestimmungen kann zur Leistungsfähigkeit grammatischer Modelle beitragen."

$54 \mathrm{Vgl}$. hierzu die Kritik von Bartsch 72, S. 9, $172 \mathrm{ff}$ an Lakoffs undifferenzierter Satzeinbettung 
5 .

Zur Relevanz der kommunikativen Intention

5.1 .

Wahl kondensierter strukturen

Die kommunikative Intention des Sprechers ist zunächst einmal ausschlaggebend dafür, daß überhaupt bei vorausgesetztem identischem Denotat gerade Partizipial- und Gerundialstrukturen statt anderer potentiell äquivalenter Formen des sprachlichen Ausdrucks gewählt werden mit dem Ergebnis einer fakultativen Verknüpfung mehrerer Prädikationen in einem Satz.

Der fakultative Gebrauch von Partizipial- und Gerundialstrukturen im Satz hat Ursachen, die über die syntaktische Ebene des Satzes hinausverweisen in den Bereich der in der Kommunikation konstituierten struktur des Textes und $z u$ umschreiben sind als Organisation des außersprachlich Bezeichneten zur sprachlichen Bedeutung gemäß der jeweiligen Sprecherintention. Grundsätzlich ist auszugehen von der funktionalen Relevanz der wahl zwischen potentiell äquivalenten strukturen; ${ }^{1}$ so kann die aktuale Gliederung relevant werden für die wahl zwischen konkurrierenden Formen der kondensierten oder expliziten Prädikation und ihrer Verknüpfung. ${ }^{2}$ Die Transformationen in der "klassischen" TG geben uber die funktionale Relevanz dieser wahl keine Auskunft.

Daß Transformationen wie Deprädikation und Einfügung in einen dann übergeordneten Satz hier nur beschrieben, nicht aber hinsichtlich ihrer spezifischen Funktion erörtert werden, hängt wesentlich zusammen mit der Beschränkung der TG auf die Satzdomäne und, in Zusammenhang hiermit, der Zuordnung des mit dem stichwort "aktuale Gliederung" umschriebenen Komplexes nicht

1 Vgl. Zimek 66, S. 69: "My polagaem, cto bez funkcional'noj motivirovki ne proizchodilo by nikakoj transformacii, ona byla by lišena smysla."; vgl. Flier 70, S. 195; Gabka 76, S. 214; Gajsina 71, S. 36ff; Meyer 75, S. $42 \mathrm{ff}$

2 Zur Relevanz der aktualen Gliederung für die Wahl zwischen Hypotaxe und Parataxe vgl. Dressler in Danes 74 (ed.). S. 98ff. Ceremisina 71, S. 32ff; zu kondensierten Strukturen vgl. Sgall u.a. 73, s. $139 \mathrm{ff}, 151 \mathrm{ff}, 250$ und die dort angeführte Literatur 
zur Tiefenstruktur, sondern als Performanzerscheinung zur oberfläche bei zugrundegelegtem identischem Denotat. ${ }^{3}$

Daß im übrigen auch hinsichtlich des Denotats nicht Äquivalenz gelten muß bei der Wahl einer zweibasigen Struktur im Sinne der TG statt einer Folge von Sätzen, zeigt das folgende Beispiel :

a) Peter ist krank. Deshalb geht er zum Arzt.

b) Peter geht zum Arzt wegen seiner Krankheit.

Nur in a) ist Peters Krankheit vom Sprecher als Tatsache behauptet, in b) kann Peter tatsächlich krank sein, aber auch nur vorgeblich oder vermeintlich. ${ }^{4}$

5.2. Interpretation der Satzkonnexion

Bei der Interpretation oberflächensyntaktisch ambiger Strukturen im Satz greift der Hörer (bzw. Leser) zurück auf die angenommene oder doch immerhin als möglich unterstellte kommunikative Intention des Sprechers, wie sie erschlossen wird aus dem Zusammenhang von Kontext und Situation und wie sie sich konstituiert auf der Grundlage der für den Sprecher in der Kommunikation relevanten Voraussetzungen. Auch die Explikation ambiger Partizipial- und Gerundialstrukturen anhand von Paraphrasen und Ubersetzungen stützt sich, meist intuitiv, auf angenommene Voraussetzungen der Mitteilungsabsicht des Sprechers. vgl. Bartsch 72, s. $110:^{5}$

"Wegen der Verkürzung und der Vermeidung unnuitzer Redundanz ist die Verwendung relationaler Adverbiale guinstig

3 Zur Kritik an der TG aufgrund der funktionalen Relevanz von Transformationen vgl. 2.B. Coseriu 75, S. $126 \mathrm{ff}$, Petöfi 71, S. 208ff, 251ff, Meyer 75, S. 16ff, Firbas in Danes 74 (ed.), S. $27 \mathrm{ff}$, Daneś a.a.O.. S. $117 \mathrm{ff;}$ zum Begriff der Transformation vgl. auch Sgall in $2 \mathrm{fSl} 75, \mathrm{~S} .783 \mathrm{ff}$

4 Vgl. Sgall u.a. 73, S. 157; Beispiele nach Bartsch 72, S. 5, Bartsch selbst geht hier nicht auf diese Problematik ein

$5 \mathrm{Vgl}$. Rúźička 73 passim; Bungarten 76, S. 153ff; Sgall in Danes 74 (ed.), S. 66; zu impliziten Satzverknüpfungsrelationen vgl. auch Meyer 75, S. $59 \mathrm{ff}$ 
in allen Situationen, in denen die relevanten Informationen bei Sprecher und Hörer gemeinsam vorauszusetzen sind -sei es durch im Text vorweg gegebene Informationen oder durch einen bei Sprecher und Hörer ziemlich homogenen Informations- und Interessenstand.

Die Distribution von relationalen Adverbialkonstruktionen im Verhältnis zu den relationalen Satzkonnektiv-Konstruktionen parataktischer und hypotaktischer Art ist zu berücksichtigen bei Untersuchungen zur Text-Konstitution und $\mathrm{zu}$ Sprecherstrategien."

Kondensierte Formen der Prädikation sind ein wesentliches Mittel zum Aufbau der Textstruktur, indem sie die Möglichkeit bieten, innerhalb des Satzes zurückzugreifen auf im Text Vorerwähntes oder doch durch das Vorverständnis des Sprechers Unterstelltes. 6

Der in diesem Zusammenhang fällige Begriff der Präsupposition bedürfte seinerseits der Präzisierung. ${ }^{7}$ sgall trifft (in ZfSl 75. S. 784ff) folgende Differenzierung:

- Präsupposition im engeren Sinn (auch bei Negation des Satzes gilt die deprädizierte Voraussetzung)

- Allegation (bei Negation des Satzes ist die deprädizierte Information weder vorausgesetzt noch negiert)

- die deprädizierte Information ist auch bei positivem Satz (im Konditional) nur möglich, nicht aber als wahr vorausgesetzt

Dringend nötig wäre also, auch wenn sich unsere Untersuchung auf den Satz beschränkt, die Untersuchung kondensierter Strukturen im Bereich des Kontextes im weitesten Sinne. Hier stellen sich jedoch vorläufig erhebliche methodologische Probleme. 8 In unserem zusammenhang soll z.B. die Abgrenzung zwischen Textlinguistik und Pragmatik nicht weiter erörtert werden, ebensowenig die Frage, ob die Texttheoerie notwendige Voraus-

6 Vgl. z.B. Dressler 72, S. 21 zur Relevanz der Gerundialstrukturen für die Textkontiguität: Weiss 77, S. 300 ff zur Funktion poln. Gerundien als "Kulisse" mit der Geltung als "supponiert wahr"; vgl. hierzu auch Sgall in zfSl 75, S. 784, ders. u.a. 73, S. $155 \mathrm{ff}$

$7 \mathrm{Vgl}$. Weiss 77, S. 302, Anm. 396; Rủzickka, SS, S. 75ff; Frantz 74, S. 30

$8 \mathrm{Vgl.2.B}$. Weiss 77, S. 9ff; Sgall u.a. 73,5.158;Danes 74,5.106 
setzung für die Diskussion der aktualen Gliederung des Satzes ist. Für unsere Zwecke soll die These genügen, daß sich die Funktion der Partizipial- und Gerundialstrukturen im Satz zwar nicht reduzieren läßt auf ihren kontextuellen zusammenhang, daß aber ein derartiger Zusammenhang bestehen kann und im Satz seinen Reflex findet in dessen kommunikativ-semantischer Gliederung ${ }^{9}$ einschließlich der formalen Kriterien, die uiblicherweise in zusammenhang mit der aktualen Gliederung, funktionalen Satzperspektive u.ä. angeführt werden. So manifestiert sich z.B. die oben angegebene Funktion der Präsupposition ( $d . h$. Nichtbetroffenheit der Erweiterung durch die Satznegation) durch die stellung der kondensierten Form in der aktualen Gliederung des Satzes. 10

Vgl. auch Firbas in Danes 74 (ed.), S. 36

".... that is not on the level of grammatical structure, but on the level of FSP that the communicative purpose of an utterance is decided."

Auf die Relevanz textueller Zusammenhänge für die Konstituierung der Funktionstypen deutscher Patizipialstrukturen im Satz verweist implizit auch Bungarten 76 mit seinen statistischen Beobachtungen zur "unterschiedlichen syntaktisch-semantischen Qualität des Akteurs bei adverbial und attributiv $z u$ interpretierenden PKKI". 11 Demnach tritt bei attributiver Funktion selten ein Pronomen als "Akteur" auf, häufig nomen appellativum, bei adverbialer Funktion umgekehrt selten nomen app. und häufig Pronomen.

Ohne daß Bungarten selbst hierauf eingeht, auf die funktionstypkonstituierende Relevanz der aktualen Gliederung stützt er sich

9 Zur kontextuellen Interdependenz der aktualen Gliederung des Satzes vgl. Pisarkova 75, S. 698ff, Frantz 74, S. 31, Raspopov 70, S. 104ff, 109ff, Petöfi 71, S. 248ff, Sgallu.a. 73. S. $155 \mathrm{ff}$, ders. in Dahl 74 (ed.), S. 25ff, Bartsch 72, S. 6, Halliday in Danes 74 (ed.), S. 44, 52ff

$10 \mathrm{Vgl}$. auch sgall in Danes 74 (ed.), S. 61ff, 64ff; a.a.0. Novak. S. 176 zur Relevanz der aktualen Gliederung für die Untersuchung sprachlichen Mißverstehens

11 Bungarten 76 , S. $166 \mathrm{ff}$ 
ja selbst, insofern die Wortstellung hier das maßgebliche Kriterium der Klassifizierung nach attributiver : adverbialer Funktion ist. ${ }^{12}$ Als ambig nennt er lediglich den Stellungstyp $\mathrm{V}+\mathrm{N}+\mathrm{I}+\mathrm{P}$.

Explizit nennt Kłizková ${ }^{13}$ die aktuale Gliederung als maßgebliche Bedingung für die Transposition nichtrestriktiver Strukturen aus der Position des Subjektdeterminanten in den Prädikatskomplex als "predikativnyj opredelitel"". Insofern das Gerund schon durch seine morphologische Form die Nichtzugehörigkeit zum Subjektskomplex signalisiert, ist damit zugleich auf die Relevanz der aktualen Gliederung für die Realisierung der asymmetrischen Korrelation zwischen Partizip und Gerund verwiesen. Die aktuale Gliederung wird hier relevant für die Position der Einbettung in einer syntaktisch formulierten Konstituentenstruktur.

Vgl. Kl1zková 69, PO, S. 26:

"Sledovatel'no, kak obosoblennyj atribut, tak i obosoblennoe Po predstavljajut soboj polupredikativnye konstrukcii togo Ke ranga, otličjusčiesja drug ot druga lis. aktual 'nym tleneniem."

Dane $x^{14}$ erörtert als Ubergangserscheinung zwischen Satz und Text Satzstrukturen, die erweitert sind durch kondensierte Strukturen: die innere Kohärenz von Texten ist wesentlich zu beschreiben anhand von Typen der thematischen Progression als Formen der Beziehung zwischen den Themata der aufeinanderfolgenden Außerungen; analoge Beziehungen können innerhalb von Außerungen auftreten.

vgl. Danes in Danes 74 (ed.), S. 115:

$12 \mathrm{Vgl}$. Bungarten 76, S. 32, 157, 226

$13 \mathrm{Vgl}$. Kłizková 69, Po, S. 6ff, 28ff; dies. 69, Synt. ch., S. 124; Adamec II 75, S. 184

$14 \mathrm{Vgl}$. Danes in Danes 74 (ed.), S. 106-128, insb. die Ubersicht S. 122-123; vgl. hierzu 2.B. Meyer 75, S. 20ff ; Sgall u.a. 73, S. 156ff; Firbas in Danes 74 (ed.), S. 27; vgl. allgemein zu Formen des "sloznoe sintaksizeskoe celoe" 2.B. Loseva 67, Kol jadenko 72,S. $89 \mathrm{ff}$ mit Hinweis auf die Relevanz des Textes für die Analyse des Satzes 
"Before starting with the classification of TP's, let us state that between a simple utterance (i.e.. a sentence containing only one $T-R$ nexus, with simple $T$ and $R$ ) and a textual concatenation of grammatically independent sentences, a transitional zone exists, comprising cases of sentence units that reveal a more complicated (condensed or composed) T-R structure, i.e.. units, that, from the point of view of FSP, reveal a textual character, which, however, represent a single grammatical unit, one sentence only."

Vgl. auch die Beispiele a.a.0. S. 117:

"Condensed U's":

a) U with a complex T: This dark-colored liquid, known as crude petroleum or crude oil, is obcained from wells of different depth. b) U with a complex R: The amino acides are required for making proteins, consisting of long chains of these units.

Der Typ der thematischen Progression wird relevant für das Auftreten von kondensierten Strukturen im Satz. Die Stellung umgekehrt von z.B. Partizipial- und Gerundialstrukturen in der aktualen Gliederung des Satzes verweist auf zumindest mögliche Textstrukturen.

Vgl. Daně̌ a.a.o. S. 116:

"Generally speaking: In respect to their T-R structure, utterances (U's) may be devided into simple U's, composed U's, and condensed U's. - The composed U's result from composition, by which two (or more) simple utterances are combined in a single sentence frame; if the $T$ ' $s$, or R's of the two utterances are the same (from the semantic point of view), they will be mentioned only once. The condensed U's are based on fusion: If two subsequent simple $U_{1}$ and $U_{2}$ share a common FSP-element, they may be fused into a sifgle condensed $U$, either by way of thematization, or rhematization of one of the utterances. The two possibilities depend on the type of the thematic interrelations obtaining between $U_{1}$ and $U_{2} \cdot "$

Während Daneš Sätze mit kondensierten Strukturen in Analogie zu Textstrukturen betrachtet, wird umgekehrt in der TG, soweit überhaupt $z u$ Texten Aussagen gemacht werden, versucht. Texte, 2.B. zwei aufeinanderfolgende Sätze und als Grenzfall die Kombination von expliziter und kondensierter Prädikation in einem 
Satz, in Analogie zu einfachen Sätzen mit Nebensatzgliedern zu beschreiben. Die Tatsache und die Position der Verknüpfung werden bei der Ableitung von zweibasigen Strukturen vorausgesetzt. Damit wird zunächst einmal über das zugrundeliegende Modell Auskunft gegeben. Es ist nicht von vornherein gesichert, daß diese Analogie gilt und zur Spezifizierung ausreicht, daß im einfachen Satz die Beziehungen beschreibbar sind, die für eine Satzverknüpfungsrelation ${ }^{15}$ ind damit auch für die spezifizierung untergeordnet prädikativer Partizipial- und Gerundialstrukturen relevant sind. Sätze mit Partizipial- und Gerundialstrukturen sind Beispiele für den stufenweisen Ubergang vom Satz zum Text bzw. lassen sich umgekehrt auffassen als Grenzfall einer Textstruktur in der Form eines Satzes. Die Beschreibung in Analogie zu vorausgesetzten Satzgliedpositionen betrifft das (oberflächen-) syntaktische Ergebnis der fakultativen Verknüpfung von Prädikationen und in diesem Sinn ist sie nützlich. Das eigentliche Problem aber ist die zugrundeliegende Verknüpfungsrelation als Grenzfall einer durch die Sprecherintention konstituierten semantischen Textstruktur, die in einem linguistischen Modell der Satzstruktur vorzuordnen wäre. 16 vgl. Bartsch 72, S. $6 \mathrm{zu}$ z.B.:

"(1) Peter geht zum Arzt wegen seiner Krankheit. (3) Peter ist krank. Deshalb (daher) geht er zum Arzt. Wegen der Verhältnisse der Texte (1) - (4) zueinander ist eine logische Analyse nicht einfach eine Analyse von voneinander isolierten Sätzen, sondern erstreckt sich zumindest auf Texte und sogar auf nicht explizierte Vorausset-

$15 \mathrm{Vgl}$ die Kritik an der TG bei Meyer 75, S. 37ff, 39ff, 41ff, Petöfi 71, S. 198ff, 208, 251; a.a.0. S. 248: "Die Beschränkung auf die Satzdomäne ist demnach graduell immer weniger zureichend für die folgenden Strukturen: - einfache Sätze ohne eingebettete Konstituenten, - Sätze mit Einbettung in der nominalen Determinante ("Rel"), - Sätze mit anderen Einbettungen als "Rel", wobei innerhalb dieser Gruppe wieder eine Reihenfolge besteht, - Sätze mit beigeordneter Verkettungstransformation": S. 250: "Die im engen Sinne genommene Satzdomäne geht also stufenweise in "Text" über, und dieser stufenweise Ubergang ist in erster Linie Folge von Ursachen semantischer Art."

16 Vgl. Grochowski 75, S. 681; Petöfi 71, S. 213, 254; Dozorec 62 ; Ceremisina 71 
zung. Der Text (3), der aus zwei Sätzen besteht, hat dieselbe logisch-semantisch zugrundeliegende struktur wie Satz (1). Daher sollte man festhalten: Gegenstand der logischen Analyse sind Texte. Im Sonderfall sind diese Texte Sätze."

Sätze mit Partizipial- und Gerundialstrukturen haben mit zwei aufeinanderfolgenden Sätzen gemeinsam, daß der Inhalt der Satzverknüpfungsrelation nicht oder doch bei weitem nicht in jedem Fall expliziert ist. Diese oberflächensyntaktische Unbestimmtheit der Konnexion ist aber noch kein Grund, uberhaupt zu resignieren hinsichtlich der Unterscheidbarkeit von Typen der Konnexion, die für die Kommunikation relevant werden.

5.3 . Konstituierung der sprachlichen Bedeutung

Auch in der syntaktisch orientierten Literatur werden implizit Hinweise auf die kommunikativ-semantische Spezifizierung gegeben in Zusammenhang mit der Signifikanz der formalen Kriterien Wortstellung und Isolierung, der grundsätzlichen Ambiguität kondensierter strukturen und ihrem fakultativen Charakter. insofern sie in Konkurrenz stehen mit potentiell äquivalenten Formen der Prädikation im Satz und im Text, und mit den semantischen Merkmalen der (untergeordneten) Prädikativität, der Nichtrestriktivität und des syntagmauberschreitenden Bezuges auf Subjekt und Prädikat gleichzeitig bzw. auf das Satzganze: damit wird in den syntaktischen Klassifizierungen von Funktionstypen der Partizipial- und Gerundialstrukturen das Nichtausreichen einer auf die syntaktische Ebene des Satzes begrenzten Betrachtung deutlich. Eine systematische Zuordnung formaler und semantischer strukturkriterien ist nicht möglich auf der Grundlage von Satzgliedpositionen, die formale und semantische Gesichtspunkte vermischen und ihre semantischen Implikationen nicht definieren, sie ist u.E. auch nicht möglich, solange Funktionstypen begrundet werden durch die semantische Charakteristik der beteiligten Lexeme einschließlich ihrer Dekomposition und (logisch-) semantische strukturen aus dem Be- 
reich des außersprachlich Bezeichneten.

Aussichten auf die zuordnung formaler und semantischer Kriterien im sinne der asymmetrischen zeichenrelation ${ }^{17}$ eröffnen sich allenfalls dann, wenn strukturen der (einzel-) sprachlichen Bedeutung, wie sie in der Kommunikation intendiert und verstanden wird, zugrundegelegt werden.

Nur: Status und Form der Darstellung dieser Bedeutungsstrukturen bedürfen noch der Untersuchung; die Diskussion hierzu ist in vollem Gange, ${ }^{18}$ ihre Ergebnisse haben durchaus vorläufigen Charakter. Hier können notgedrungen nur einige Anmerkungen gegeben werden. Unsere Aufgabe ist nicht die Etablierung eines Geschlossenheit vorgebenden linguistischen Modells. Für unsere Zwecke soll in einer höchst provisorischen Weise zwischen den Ebenen der oberflächensyntaktischen und der semantischen Repräsentation unterschieden werden. Erstere kann ambig sein. die semantische Repräsentation ist explizit und eindeutig. Die oberflächensyntaktische Ebene ist linear strukturiert. die semantische durch die hierarchische Zuordnung von Prädika-. tionen.

Die Ebene der semantischen Repräsentation betrifft nicht Strukturen des außersprachlich Bezeichneten, sondern der sprachlichen Bedeutung, wie sie in der Kommunikation mit ihren vielfältigen Bedingungen vom Sprecher intendiert und mitgeteilt wird. Natürlich ist damit nicht bestritten, daß Mitteilungen über außersprachliche Sachverhalte gemacht werden können, nur bestimmen grundsätzlich nicht diese selbst die Bedeutungsstruktur der Mitteilung, vielmehr ist diese das Ergebnis der Organisierung des Bezeichneten durch den Sprecher (bzw. Hörer) in

$17 \mathrm{Vgl}$. Prager Autorengruppe 75, S. 80, $121 \mathrm{ff} \mathrm{mit}$ Hinweis auf Hjelmslev und Lamb; Sgall u.a. 73, S. 74, 124ff; Danes u.a. 75. S. 616

$18 \mathrm{Vgl}$. z.B. mit den wesentlichen Literaturangaben Weiss 77, S. 19ff "Was beschreibt eine Tiefenstruktur?"; vgl. auch a.a. O. S. 21ff, S. 25; Rohrer 71, S. 17ff, 106ff; Bartsch/v. 72, S. 4ff; ZfSl 75 passim, insbes. Danes u.a., S. 613ff; Sgall u.a. 73; Prager Autorengruppe 75; Dahl 74 (ed.): Danes 74 (ed.): Immler 74; Svedova 74, S. $90 f f$ 
der Kommunikation. 19 Die Bedingungen der Konstituierung der sprachlichen Bedeutung verweisen iber die Satzgrenze hinaus auf Kontext und Situation im weitesten Sinne, lassen sich aber nicht reduzieren auf Faktoren außerhalb des Satzes. Diese in der Kommunikation uibermittelte Bedeutung hat zunächst einmal einzelsprachlichen Charakter. 20 Ihre struktur wird wesentlich beschrieben anhand der unter dem Oberbegriff "aktuale Gliederung" entwickelten Terminologie. Bei aller Vorläufigkeit ${ }^{21}$ der Diskussion heißt dies, daß eben die aktuale Gliederung Teil der semantischen Repräsentation ist. 22 Auch $z$. B. Saumjan ordnet den "semantischen Akzent", dessen Kriterium wesentlich die Wortstellung ist, dem Bereich der genotypischen, nicht der phänotypischen strukturen $z u$.

Demzufolge sind Erscheinungen der aktualen Gliederung nicht hinreichend $z u$ behandeln, wenn man sie als Erscheinungen der Performanz der oberfläche zuordnet: ${ }^{23}$ vielmehr hat die aktuale

19 Vgl. Sgall u.a. 73, S. 1lff, 74, 139; Prager Autorengruppe 75, S. $31 \mathrm{ff}, 89 \mathrm{ff}$; Rohrer 71, S. $93 \mathrm{ff}$

$20 \mathrm{Vgl}$. Sgall u.a. 73, S. 9, 75: "Meaning is language dependent."

$21 \mathrm{Vgl}$. Weiss 77, S. 49ff; Dezsö/Szépe in Dahl 74 (ed.), S. 90: "So the status of topic-comment relation cannot be considered yet as cleared up within the frame-work of language description."; vgl. ähnlich Sgall in Dahl 74 (ed.), S. 25

$22 \mathrm{Vgl}$. 2.B. Adamec I 73, S. 3ff, 128ff; Cernachovskaja 76; Dahl in Danes 74 (ed.), S. 75ff: Dahl 69: Danes u.a. in ZfSl 75, S. $621 \mathrm{ff}$; Birnbaum 70, S. 62; Abraham/Binnick 72, S. XIff; Prager Autorengruppe 75, S. 89ff: "Tektogrammatische Ebene"; Sgall u.a.73, S. 74ff, 142ff, 245ff: ders. in Danes 74 (ed.), S. 27, 55ff; Adamec a.a.0.. S. 189: "... aktual'noe clenenie pridaetsja tak ili inace vkljucit' $\checkmark$ GS poskol'ku ono otćastiopredeljaet vozmónosti obrazovanija otdel'nych poverchnostnych struktur iz dannoj glubinnoj struktury."; a.a.0. Dezsö/szépe, S. 81ff; Flier 70: zur "konzeptuellen Ebene" Bartsch 72, S. 353: Raspopov 70, S. 109, 112, 133ff

$23 \mathrm{Vgl}$. zur diesbezüglichen Kritik an Chomsky z.B. Abraham/B.72, S. 87ff, Immler 74, S. 191, Birnbaum 70, S. 67, Rohrer 71, S. 67ff, 180, Petöfi 71, S. 200ff, Sgall u.a.73, s. 9ff, 27. $161 \mathrm{ff}, 240$, Meyer 75, S. 16, Dahl in Danes 74 (ed.), S. 75. Dressler a.a.0., S. $96 \mathrm{ff}$ 
Gliederung systematischen Charakter und mit organisierende Relevanz für die Satzbedeutung. Dies kann sehr wohl Konsequenzen haben für die oberflächensyntaktische Gliederung 24 sowie für die morphologische Form der oberfläche. Die Schwierigkeiten, die sich für die "klassische" TG wie für die logische Syntaxtradition überhaupt und für die Anwendung eines ausschließlich an Wahrheitswerten orientierten Prädikatenkalküls ergeben bei der Erörterung von Fragen der aktualen Gliederung, gehen letztlich zurück auf den nicht geklärten Begriff der sprachlichen Bedeutung.

Es war einer der zentralen Einwände gegen die "klassische" TG, daß der für verschiedene Oberflächenstrukturen mit angenommener gemeinsamer "Tiefenstruktur" vorausgesetzte Begriff der linguist.Identität nicht präzisiert wird. Fundiert ist er allenfalls durch die Identität des außersprachlich Bezeichneten, 25 nicht aber durch die Identität der sprachlichen Bedeutung. Diese wäre im Idealfall zu fordern für die Begründung intra- und interlingualer Aquivalenzen.

Kommunikative Indifferenz und orientierung am kognitiven Inhalt gilt auch für zumindest die Anfänge der Generativen Semantik. 26 Dies läßt sich in unserem Zusammenhang zeigen für den auf lexikalischer Dekomposition beruhenden Begriff der impliziten Prädikation bei starikova 74, der nicht geeignet ist, zu unterscheiden zwischen in kommunikativem sinne untergeordnet prädikativen und nichtprädikativen strukturen, sowie für die Nichtdifferenzierung des satzsemantischen Unterschiedes zwischen Satzadverb und Modaladverb bei gleichem lexikalisch-semanti-

24 Vgl. auch z.B. Sgall u.a. 73, S. 27: Pala in Danes 74 (ed.) S. 199; Kłiżková 69, PO

$25 \mathrm{Vgl}$. zur Kritik am Semantikbegriff in der TG 2.B. Coseriu 70 S. $69 \mathrm{ff}$, ders. 75, s. $26,122 \mathrm{ff}, 126 \mathrm{ff}$, Weiss 77, s. 19ff, Immler 74, S. 192, Rohrer 71, S. 93ff, a.a.0. S. 216, Anm. 22: "Die Definition des Begriffes der linguistischen Identität ist übrigens eines der gröBten offenen Probleme der TG. Die bisherigen Definitionen mit Hilfe der Definentia "lexikalische Identität", "referentielle Identität", "Identität der Baumstruktur" haben sich als inadäquat erwiesen."; vgl. auch Sgall u.a.73, S. 181ff, Petöfi 71, S. 248ff, 254

26 Frantz 74.S.22.28ff, Sgall u.a.73,S.243, Prager Autorengruppe 75.S.57ff, Meyer 75, S.37ff 
schen Inventar bei Lakoff. 27

Nur der Bereich des uhereinzelsprachlich-außersprachlich Bezeichneten, nicht aber die in der Kommunikation konstituierte (einzel-) sprachliche Bedeutung ist mit den Mitteln eines nicht modifizierten Prädikatenkalküls darzustellen. ${ }^{28}$ Dem entspricht es z.B. auch, wenn F. Schmidt den "subjektiven Akzent" nur am Rande erwähnt und bei vorausgesetzter syntaktischer struktur die gleiche prädikatenlogische struktur fiir unterschiedlichen "subjektiven Akzent" anfuhrt. 29

Vgl. demgegenuiber Sgall in Dahl 74 (ed.), S. 25:

"The impact of these units, i.e. the basic relevance of topic/comment articulations (TCA) in a language, its necessity and the intrinsic nature is connected with the fact that the semantic structure of the sentence differs froma logical formula in that it is a unit adapted to functioning in the communication."

Vgl. Sgall u.a.73, S. 144:

"We have found, furthermore, that it is useful to consider a coincidence in truth conditions necessary, but not sufficient for the identity of the linguistic meaning of sentences.... since if two sentences differ in the position of $B J$ or in $C D$, they differ, in general case, also in what is asserted and what is presupposed in them, and so they differ in their conditions of use, i.e. they are no genuine paraphrases and cannot be used in the same contexts, nor embedded in the same matrix sentences (without influencing the semantics of the whole complex sentence)."

$(C D=$ communicative dynamism, $B J=$ boundness juncture Ch. H.: vgl. zu diesen Begriffen Sgall u.a.73, S. 45ff)

Relevant für die wahl und Interpretation der Partizipial- und Gerundialstrukturen ist nicht das außersprachlich Bezeichnete. sondern die die sprachliche Bedeutung konstituierende kommunikative Intention des Sprechers.

$27 \mathrm{Vgl}$. Bartsch/V. 72, S. 10ff, 18ff, 32

$28 \mathrm{Vgl}$. Weiss 77, S. 21ff: "Die Logisierung der grammatischen Beschreibung"; Bartsch/V. 72, S. 26; Lomtev 72, S. 196ff: Allwood 73, S. 102: Rohrer 71, S. 96ff, 175ff; Sgall u.a.73, S. $122 \mathrm{ff}, 181 \mathrm{ff}$

$29 \mathrm{Vgl}$. Schmidt, F. 70, S. $18 \mathrm{ff}, 46 \mathrm{ff}$; vgl. demgegentiber z.B. Bartsch 72, S. 68ff, 73ff: 
Anhand der sprachlichen Bedeutung, nicht aber des auBersprachlich Bezeichneten sind die Funktionstypen dieser fakultativen und ambigen strukturen $\mathrm{zu}$ bestimmen. 
Kennzeichnend für "attributive Funktion" des russischen Partizips ist, daß sich die funktionstypkonstituierenden semantischen Beziehungen erschöpfen in der Bestimmung der übergeordneten Nominalphrase: 1 die Einschränkung "funktionstypkonstituierend" soll besagen, daß zusätzliche semantische Assoziationen verschiedenster Art nicht in jedem Fall auszuschließen sind, ${ }^{2}$ daß jedoch auf keinen Fall eine Beziehung zum Prädikat relevant wird für den Funktionstyp selbst. Die nicht auszuschließenden gelegentlich erwähnten zusätzlichen Beziehungen sind zur spezifizierung der attributiven Funktion selbst nicht geeignet. Explikation erfolgt durch Paraphrase mit Relativsatz, dies entspricht der ausschließlichen Beschreibbarkeit im Rahmen der Nominalphrase. Diese einseitige Beziehung der Unterordnung, die sich innerhalb des Syntagmas mit dem regierenden (Pro-) Nomen erschöpft, ist auch durch Weglaßprobe zu veranschaulichen: die ubergeordnete Komponente kann ohne die untergeordnete stehen, nicht aber umgekehrt.

Diese Bestimmung gilt sowohl für "Attribut" als auch für "Apposition". wie sie in der Literatur nach im einzelnen wechselnden Kriterien (Wortart, restriktiv : nichtrestriktiv, wortstellung vor/nach Bezugsnomen, mit/ohne Isolierung) unterschieden werden. Wir verwenden nur den Terminus "attributiv" zur

$1 \mathrm{Vgl}$. Weiss 77 , S. $81 \mathrm{ff}$ und die dort angefuhrte Literatur,a.a. O. S. 82: "Attribute spezifizieren NPs": Heine 72, S. 218 : Adamec II 75 , S. 29ff; Rath 71, S. 50ff, 128ff, $137 \mathrm{ff;}$ RúZicka, SS, S. 232ff, 272ff, 282ff, $289 \mathrm{ff}$

$2 \mathrm{Vgl}$. Musić 35, S. 128ff: "Atributivni pridjev cini sa svojom imenicom cjelinu, te onom sto se glagolom izrice, izrice se o imenice zajedno s pridjevom: ....Ali na predikat moze atributivni pridjev utjecat, jer mu moze znaciti uzrok, uvjet itd.. ali se ne pridijeva zato, nego propada joj bez obzira na predikat...": Rohrer 71, S. 193ff: Francuk 52, S. 15; Rudnev 68 , S. 151; Weiss 77, S. $88 f f$ 
Kennzeichnung der Beschreibbarkeit ausschlieblich im Rahmen der NP, um Mißverständnisse zu vermeiden, die sich ergeben könnten aufgrund der außerdem in der Literatur z.T. uiblichen Verwendung der Bezeichnung "Apposition" auch noch für Funktionstypen, für die der Bezug auf Subjekt und Prädikat gleichzeitig konstitutiv ist ${ }^{3}$.

Vgl. zum einseitigen Bezug der aksl. nichtprädikativen (attributiven) Langform Rúżicka, sS, S. 282:

"Eines der wichtigsten Kennzeichen des merkmalhaltigen nichtprädikativen Partizips gegenüber dem merkmallosen besteht darin, daß die Kategorie des Aspekts und des Tempus sich nicht als direkte Charakterisierung des Verhältnisses der Handlung des Partizips zur Handlung des Hauptverbs auswirken können. Dieses Verhalten ergibt sich notwendig aus dem Merkmal der Nichtprädikativität. Lassen sich Partizip und Hauptverb in der Prädikation vereinigen (Kurzpartizip), so müssen sich Aspekt und Tempus des Partizips als Bestimmung des Verhältnisses seiner Handlung zur Haupthandlung geltend machen. Ein temporaler Nebensatz ist für ein Vollpartizip in keinem Falle eine angemessene Ubersetzung."

Eindeutig bezeichnet ist die Nichtprädikativität im Aksl. durch die Langform des Partizips; das russische Partizip kann auch untergeordnet prädikativ fungieren, wie umgekehrt im Aksl. mit der merkmallosen Kurzform in nichtprädikativer Funktion zu rechnen ist.

6.2. Der funktionale Gegensatz restriktiv : nichtrestriktiv und seine Kennzeichnung

Nichtprädikativität, d.h. die Funktion als Referenzakt zum

3 so z.B. bei Vondrák 28, S. 402, Miklosich, Musić und noch bei Weiss 77, S. 227ff, 352ff, wo mit "Apposition" gerade nicht einseitige Unterordnung unter regierende NP gemeint ist, sondern gleichzeitiger Bezug auch auf das Prädikat im Sinne sekundär-prädikativer Strukturen bei Rủzicka 66; vgl. auch ders.. SS, S. 137 kritisch zur "Apposition" bei Potebnja und Ovsjaniko-Kulikovskij: vgl. Pesikan 59 zur Auseinandersetzung um die Termini appositiv : adverbial sowie die Polemik gegen Musić bei Belić 33/34 
Zweck der Identifikation, ist maximal ausgeprägt bei Restriktivität der Beziehung zwischen Attribut und Bezugsnomen. Bei Nichtrestriktivität liegt demgegenüber graduell abgestufte Prädikativität vor, wobei jedoch ebenfalls Bezug ausschlieblich auf die übergeordnete Nominalphrase und damit Beschreibbarkeit im Rahmen der NP gegeben ist. 4

Klassenlogisch schränkt ein restriktives Attribut die Extension des mit dem übergeordneten $\mathrm{N}$ bezeichneten Begriffes ein, ein nichtrestriktives nicht. 5

Seiler 60, s. $21^{6}$ veranschaulicht dies graphisch so:
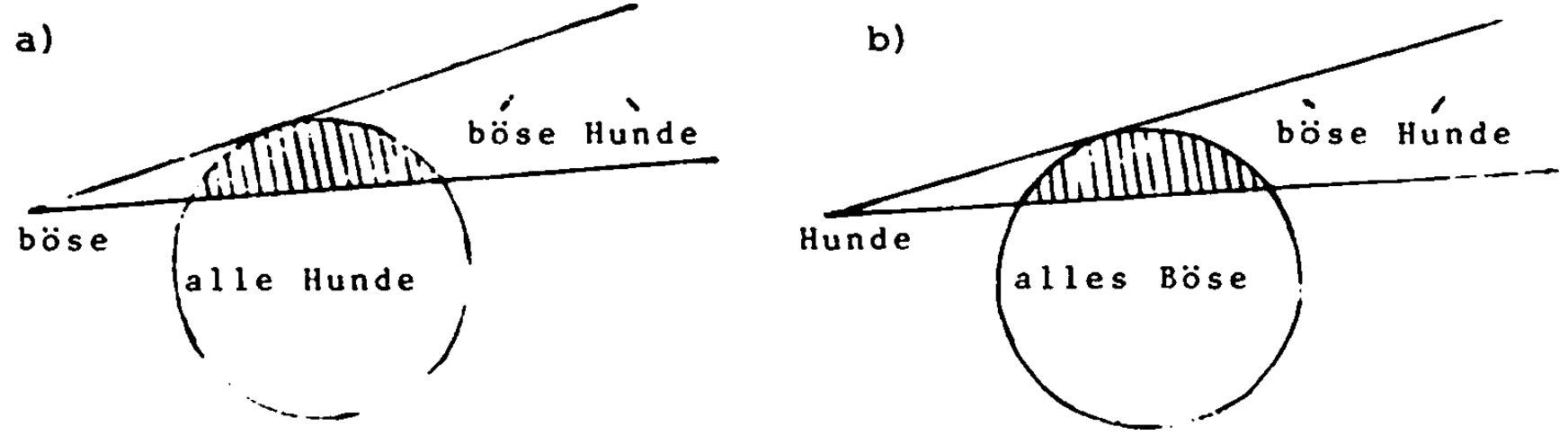

In a) ist "böse" restriktiv, in b) nicht bzw. in Seilers Terminologie: in a) (Spezifikation) ist "böse" Selektor zur Klasse "Hunde", in b) (Charakterisierung) umgekehrt "Hunde" Selektor zur Klasse "böse". Die Relation Klasse - Selektor tritt nicht nur im Rahmen des Nominalkomplexes auf.

Konstituiert wird der Gegensatz restriktiv : nichtrestriktiv nicht durch die außersprachliche wirklichkeit oder durch syntaktische Fügungseigenschaften: 7 letzteres ist evident im Hinblick auf die Beziehung zum Nomen, spielt aber eine Rolle für die Bestimmung von Funktionstypen im Rahmen des Prädikatskomplexes.

4 Vgl. Valgina 72, S. 101; Weiss 77, S. 356

$5 \mathrm{Vgl}$. Rohrer 71, S. 137ff, 204ff, $256 \mathrm{ff}$ und, ihm folgend, Weiss 77. S. $87 f f$

$6 \mathrm{Vgl}$. Seiler 60, S. $9 \mathrm{ff}, 23 \mathrm{ff}, 35 \mathrm{ff}$

7 dies auch zu Adamec II 75, S. $7 \mathrm{ff}, 32 \mathrm{ff}, 170 \mathrm{ff}, 184 \mathrm{ff}$ : Rath 71. S. 125; Bungarten 76, S. $205 \mathrm{ff}$ 
Hier wird in der Literatur zum "prädikativen Determinanten" u.ä. nicht immer exakt unterschieden zwischen syntaktischer und kommunikativer Fakultativität. Syntaktische Fakultativität, d.h. vereinfacht Nichtbedingtheit durch Fügungseigenschaften der uibergeordneten Konstituente, z.B. des Verbums, ist allenfalls notwendige, nicht aber hinreichende Bedingung für kommunikative Fakultativität (Nichtrestriktivität).

Die Bedingungen der Konstituierung des Gegensatzes restriktiv : nichtrestriktiv liegen im Bereich des kommunikativen zusamenhangs der Außerung. Sie betreffen die Voraussetzungen, die der Sprecher bei möglicher Identität des außersprachlich Bezeichneten in der Kommunikation zugrundelegt im Hinblick auf das unterstellte Vorwissen des Hörers. ${ }^{8}$ D.h.:Unabhängig davon, ob das nichtrestriktive Partizip (oder Gerund) mitgeteilt wird oder nicht, ist das Bezugsnomen (bzw. die Prädikatshandlung) durch den Hörer als vom Sprecher gemeint identifizierbar; insofern ist die zusätzliche Mitteilung kommunikativ fakultativ; doch nur bei Nennung des restriktiven Partizips ist das Bezugsnomen überhaupt als das vom sprecher gemeinte zu identifizieren und insofern ist das Partizip kommunikativ obligat; analog ist nur bei Nennung des kommunikativ obligaten Gerunds im Rahmen des Prädikatskomplexes die Prädikatshandlung als die vom Sprecher intendierte zu identifizieren.

Hier soll zunächst die struktur des Nominalkomplexes erörtert werden.

Aus dem Gegensatz von kommunikativer Obligatheit/Fakultativität ergibt sich die z.B. von Weiss 77, S. 89 angeführte distributionelle Beschränkung:

"Nur nicht-restriktive Relativsätze können Eigennamen, Personalpronomina der 1 . und 2. Pers.sowie delktisch oder anaphorisch verwendete Demonstrativpronomina spezifizieren, d.h. nach Rohrer (1971), S. 205 alle Elemente, die Individuenbegriffe bezeichnen (vom Sprecher zum zeitpunkt der Außerung mit Bezug auf nur ein Objekt verwendet wer-

$8 \mathrm{Vgl}$. Grannis 75, S. 111ff; Zaza 65, S. 67ff, 71; ders. 64, S. 182,184 ; Meyer 75, S. 62; M., S. 45ff; Kłizková 68 
den), deren Extension also nicht weiter eingeschränkt werden kann."

Der Gegensatz restriktiv : nichtrestriktiv wird relevant fuir die Stellung von Partizipial- und Gerundialstrukturen in der aktualen Gliederung und deren formale Signalisierung, für syntaktische Funktionen, für mögliche Paraphrasen und für die transformationel le Beschreibung. Zugrunde liegt zunächst einmal der Gegensatz zwischen Funktion als Referenzakt zum Zwecke der Identifikation bei Restriktivität und untergeordneter Prädikativität, d.h. Charakter einer (reduzierten) Aussage in der Kommunikation, bei Nichtrestriktivität.

In dieser abgestuft prädizierenden Funktion liegt die Gemeinsamkeit nichtrestriktiver Attribute mit sekundärprädikativen Strukturen, wie sie in einer an der binären cliederung des Satzes orientierten Darstellung im Rahmen des Prädikatskomplexes beschrieben werden. Von dieser abgestuft prädizierenden Funktion $z u$ unterscheiden ist die syntaktisch untergeordnete. jedoch im kommunikativen Sinn nicht graduell abgestufte prädikation, wie sie geleistet wird z.B. durch ein finites verhum im Relativsatz: restriktiver und nichtrestriktiver Pelativsatz mögen sich durch andere Kriterien unterscheiden (z.B. Intonation, Pause, Interpunktion), nicht jedoch durch die immer aktuale Prädikation des Verbums.

\subsubsection{Intonation}

Dem "Selektor" bei Seiler 60 entspricht in der Terminologie der aktualen Gliederung "Rhema", der "Klasse" "Thema": die unterschiedliche Ausformung der Relation Klasse - Selektor im Rahmen des Nominalkomplexes, relevant für die untergeordnete Prädikativität des Partizips, manifestiert sich in der Intonation als formalem Kriterium der aktualen Gliederung. Im ein-

9 Vgl. Weiss 77, S. 83, 238ff, 246; Grannis 75, S. lliff, 116: Kornilov 67, S. 6ff 
zelnen ist das hier nicht zu erörtern; grundsätzlich ist in einer Reihe von Sprachen und auch im Russischen die Hervorhebung der restriktiven Bestimmung durch die Intonation 24 beobachten.

Vgl. zum Deutschen 2.B. die Beispiele bei Seiler 60, S. 19: bóse Hunde (restriktiv) : böse Húnde (nichtrestriktiv)

Nur der nichtrestriktive Relativsatz ist nach Bungarten 76. S. $135 \mathrm{ff}$ durch isolierte nichtrestriktive attributive Partizipialstruktur zu paraphrasieren:

Dás Heimatgefühl, das in der Regel auf den starken und nachhaltigen Eindricken der kindheit beruht, hat wenig mit Vaterlandsliebe oder Nationalität zu tun (restriktiv)

aber:

Das Heimatgefühl, in der Regel auf den starken und nachhaltigen Eindrucken der Kindheit beruhend, hat wenig mit vaterlandsliebe oder Nationalität zu tun= Das Heímatgefühl-das in der Regel auf den starken und nachhaltigen Eindrücken der Kindheit beruht - hat wenig mit Vaterlandsliebe oder Nationalität zu tun (nichtrestriktiv)

Für das Polnische zeigt Weiss 77, S. $88 \mathrm{ff}$ die intonatorische Hervorhebung restriktiver Bestimmungen und ihre prosodische Zusammenfassung mit dem Bezugswort, während nichtrestriktive Bestimmung durch Pause abgetrennt und nicht hervorgehoben wird: Podlotki majece piégi sq zawsze zakompleksione (restr.) Podlotki, majoce piegi, sq zawze zakompleksione (nichtrestr.) bzw.:

Podlotki," które majo piegi (nichtrestr., lange Pause) Podlotki, ' które maje piégi (restr., kürzere Pause, Intonationsgipfel im Relativsatz)

Auch im Russischen tritt das restriktive Attribut als Intonationszentrum in der Nominalphrase auf. ${ }^{10}$ Umgekehrt ist das nichtrestriktive Attribut weder im Rahmen der Nominalphrase noch des ganzen Satzes alleiniges Intonationszentrum. Entspre-

$10 \mathrm{Vgl}$. Kłizková 69, PO, S. 8; Rudnev 68, S. $132 \mathrm{ff}$ 
chend betont Weiss die Nichtfokussierbarkeit nichtrestriktiver Attribute. 11 Demgegenuiber liegt Fokussierung vor bei der Erweiterung einer prädikativen NP durch restriktive struktur: 12 Anka to człowiek, który jest świadom swoich obowizzków (Weiss 77, S. 90)

in dem hiervon abweichenden

Ten wysoki pan, to mój dawny profesor, którymniezreszta kiedýs oblaz na egzaminie (Weiss 77, s. 92)

ist der Relativsatz nicht restriktiv und nicht der intonatorische Gipfel des Satzes.

Andererseits sehen Weiss und Krizková für nichtattributive Strukturen im Rahmen des Prädikatskomplexes, d.h. für sogenannte sekundärprädikative Strukturen, die Möglichkeit der Fokussierung vor, offenbar unter Beibehaltung der untergeordneten Prädikativität; dies betrifft nichtisolierte Gerundien sowie Partizipien, die üblicherweise als Prädikatsnomen klassifiziert werden, 13 von Weiss aber analog $2 u$ nichtrestriktiven Strukturen abgeleitet werden. Wenn allerdings restriktive strukturen tiefenstrukturell zurückgehen auf fokussierte und in der Darstellung der Ableitungsgeschichte eine Sonderstellung einnehmen (adnominale Einbettung bei Weiss, Unmöglichkeit der Transposition aus dem Subjektskomplex in den Prädikatskomplex bei Krizková), scheint es problematisch, an der Oberfläche fokussierte strukturen auf einen gemeinsamen Ableitungspunkt zusammen mit nichtrestriktiven strukturen zuruickzufuhren.

źaźa 65 nennt demgegenüber als "restriktiv":

deti vozvrasçalis' razrumjanivsiesja (źaźa 65, S. 67)

und als "nichtrestriktiv":

deti vozvrascalis's katka tol'ko vecerom, razrumjanivsiesja i veselye

(źaza 65, S. 67)

$11 \mathrm{Vgl}$. Weiss 77, S. 9off, 357, 383

12 a.a.o. S. $90 \mathrm{ff}, 247 \mathrm{ff}$ mit Hinweis auf Motsch

13 a.a.O. S. 361ff, 367; Kłizková 69, PO, S. 10ff; zur Fokussierung des poln. Gerund vgl. Weiss 77, s. 383 
6.2.2. Paraphrasen

Die tiefenstrukturelle Relevanz des Gegensatzes restriktiv : nichtrestriktiv zeigt sich im unterschiedlichen Paraphraseverhalten. Grundlegend dabei ist, daß restriktive Attribute ausreichend beschreibbar sind im Rahmen der NP; das restriktive Syntagma ist auch außerhalb des Satzes denkbar, die nichtrestriktive Funktion z.B. des Partizips kann nur im Satz beschrieben werden. 14

Für restriktive und nichtrestriktive Partizipien im Russischen sind grundsätzlich möglich Relativsatzparaphrase zur Explikation der Einseitigkeit der funktionstypkonstituierenden Beziehungen sowie bei Nachstellung und Isolierung Permutation vor $\mathrm{N}$ und Aufhebung der Isolierung (dies allerdings mit stilistischen Einschränkungen z.B. hinsichtlich der Länge der isolierten struktur). Hinzu kommen bei nichtrestriktiven Partizipien weitere Möglichkeiten der Umwandlung, die über die Grenzen des Syntagmas hinausführen, zu einem Umbau der syntaktischen Gliederung führen und bei Restriktivität ausgeschlossen sind; vgl. die Ersetzung durch Parenthesesatz, durch koordinierte finitverbale Prädikation und durch selbständigen Satz. 15 Für die Definition der (oberflächen-)syntaktischen Position Attribut selbst dagegen ist der Gegensatz restriktiv : nichtrestriktiv nicht konstituierend, und er ist nicht relevant für die paradigmatisch orientierte Ersatzprobe (durch Relativsatz). die funktionale Unterschiede nur soweit expliziert, als sie im vorausgesetzten syntaktischen Modell enthalten sind und andere, hier relevante Unterschiede bei der Transposition auf die Satzgliedebene neutralisiert. 16

Der grundsätzliche Unterschied ergibt sich schon aus der Umschreibung der Ambiguität hinsichtlich restriktiv : nichtrestriktiv bei Rohrer 71, S. 137 :

$14 \mathrm{Vgl}$. Kornilov 67, S. $6 \mathrm{ff}$

$15 \mathrm{Vgl}$. 2.B. Rohrer 71, S. 138ff; Grannis 75, S. 112ff, 117ff; Seiler 60, S. 24ff, 27ff, 36ff, $45 \mathrm{ff}$

$16 \mathrm{Vgl}$. auch die Kritik von Grannis 75 , S.113ff an Thompson 
"So kann der folgende Satz

(69) Die fleißigen Bienen sammeln Honig entweder bedeuten, daß nur die fleiBigen Bienen Honig sammeln oder, daß alle Bienen fleiBig sind und Honig sammeln."

Zur Affinität nichtrestriktiver zu koordinierten Strukturen vgl. z.B. den folgenden aksl. Beleg, für den (wenn überhaupt) nur restriktive Interpretation in Frage kommt: 17

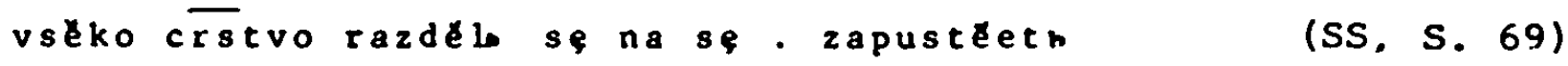

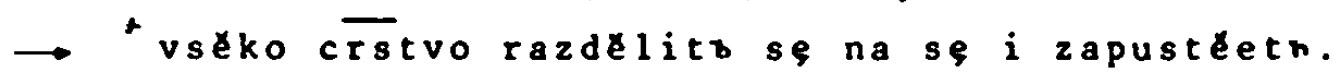

gegenüber z.B. (franz.) bei Nichtrestriktivität: 18 Même Marie, qui m'aime bien pourtant, est partie/ Même Marie est partie et pourtant elle m'aime bien

(Rohrer 71. S. 138)

Nichtrestriktive Strukturen besitzen einen vom Sprecher garantierten Wahrheitswert:

Paul nie que Marie, qui est sa meilleure amie, ait volé l'argent

(Rohrer 71, S. 138)

Hier leugnet Paul nicht, daß Marie seine beste Freundin ist. Die mit dem nichtrestriktiven Relativsatz gegebene Mitteilung ist plausiblerweise eine Aussage des von Paul verschiedenen Sprechers.

Vgl. hierzu auch das bekannte Beispiel McCawleys ${ }^{19}$ zur Ambiguität hinsichtlich der Interpretation "de dicto / de re": willy said that he has seen the woman who lives at 219 Main Street.

Die Aussage uber den wohnort der Frau macht entweder a) willy oder b) der von willy verschiedene sprecher. Nur bei b) wohnt die Frau (nach Ansicht des Sprechers) sicher in der Main street 219; in der deutschen Ubersetzung zeigt sich dieser Unterschied auch im Modus:

Willy sagte, er habe die Frau gesehen, die in 219 Main street wohne (a) / wohnt (b) 20

Nur bei b) ("de re" - Zusatz des von willy verschiedenen Spre-

$17 \mathrm{Vgl}$. Rủzicka, SS, S. 69ff, 88ff; M., S. $45 \mathrm{ff}$

$18 \mathrm{Vgl}$. Rohrer 71, S. 137ff, 187ff, 204ff, $211 \mathrm{ff}$

19 hier nach McCawley 74, S. $141 \mathrm{ff}, 149$

$20 \mathrm{Vgl}$. auch Rohrer 71, S. $205 \mathrm{ff}$ 
chers) ist Nichtrestriktivität gesichert: nur bei b) ist möglich:

Willy sah die Frau. Sie lebt in 219 Main Street. Die Frau-sie lebt in 219 Main Street-wurde von Willy gesehen. Die Frau lebt in 219 Main Street und wurde (infolge dessen) von Willy gesehen;

nur bei b), nicht bei a), wäre möglich:

Zivuscaja 219 Main Street, Zençina byla uvidena Willy. d.h.: nur bei Nichtrestriktivität des Attributs kann bei den Umwandlungen gegebenenfalls ein Bezug zwischen der Prädikatshandlung und dem paraphrasierten Attribut intendiert werden. In dem folgenden nichtrestriktiven Beispiel liegt Nachzeitigkeit der Handlung des Relativsatzes vor:

John kicked the dog, which bit him (Grannis 75, S.117) Demgegenüber ist bei Restriktivität eine Aussage über die Zeitfolge nicht möglich und auch nicht intendiert; die Handlung des restriktiven Relativsatzes kann vor-, gleich-oder nachzeitig sein:

John kicked the dog that bit him

(Grannis 75, S.117)

\subsubsection{Transformationelle Beschreibung}

Für die transformationelle Beschreibung ziehen Rohrer und Weiss aus dieser Situation die Folgerung, in der Tiefenstruktur restriktive Attribute durch adnominale Satzeinbettung, nichtrestriktive strukturen (mit subjektsbezug) aber gemeinsam mit solchen, die an der Oberfläche im Rahmen des Prädikatskomplexes als sekundärprädikativ auftreten, anhand der Konnexion von Teilsätzen durch das "logische und" 21 darzustellen. Damit trägt die Tiefenstruktur in einer sehr ökonomischen Weise dem Unterschied 2 wischen Referenzakt und graduell abgestufter Prädikativität in der Kommunikation Rechnung.

Andere Vorschläge zur transformationellen Ableitung von Attri-

$21 \mathrm{Vgl}$. Rohrer 71, S. 139ff, 187, 208ff; Weiss 77, S. 64, $246 \mathrm{ff}, 352 \mathrm{ff}, 367,384$ 
buten diskutiert Weiss ausführlich. ${ }^{22}$ Hier soll der kurze Hinweis genügen, daß z.B. Rủzicka 66 ähnlich wie Lees und Motsch zwischen restriktiven und nichtrestriktiven strukturen durch unterschiedliche Einbettungsposition unterhalb der dominierenden NP unterscheidet.

Bei Kr̉izková werden in der Ausgangsstruktur restriktive und nichtrestriktive Attribute in der gleichen Position eines adnominal als Subjektsdeterminant eingebetteten Satzes vorgesehen. 23 Der Unterschied liegt hier in der Möglichkeit nachfolgender Transformationen: nur von nichtrestriktiven strukturen kann gegebenenfalls durch Transposition in den Prädikatskomplex der isolierte oder nichtisolierte "prädikative Determinant" abgeleitet werden. Restriktive, nichtrestriktive und sekundärprädikative strukturen haben hier den gleichen Ausgangspunkt, was sich letztlich begrindet durch die außersprachlich und logisch relevante Tatsache der subjektsidentität für verschiedene Formen der aktualen und potentiellen Prädikation.

Weiss sieht demgegenüber den kommunikativen Unterschied zwischen Referenzakt und (untergeordneter) Prädikativität in der Kommunikation als grundlegend für die Unterscheidung von Satzkonnexion und adnominaler Einbettung.

Damit ist bereits vorgegriffen auf strukturen außerhalb des Subjektskomplexes. Zur Darstellung insbesondere von russischen Beispielen für attributive strukturen soll zunächst der Hinweis genügen, daB nur bei nichtrestriktiven strukturen und hier insbesondere bei der Distribution $\mathrm{N}+\mathrm{I}+\mathrm{P}$ das Problem der Ambiguität hinsichtlich der zuordnung zum Subjekts- oder Prädikatskomplex auftritt. Dies entspricht Krizkovás Hinweisen zur Nichtrestriktivität als (notwendiger) Bedingung für die Transposition in den Prädikatskomplex und bei Kornilov der Relevanz der Nichtrestriktivität für Typen der aktualen Gliederung, die die Ersetzung des Partizip durch Gerund, durch koordinierte Prädikation oder selbständigen Satz erlauben.

$22 \mathrm{Vgl}$. Weiss $77, \mathrm{~S}, 185 \mathrm{ff}$

$23 \mathrm{Vgl}$. Kứiźková 68, S. 35; dies. 69, PO; dies. 69, Synt.ch. 
6.3 . Relativsatzparaphrase

Wir untersuchen nicht die Bedingungen der Konkurrenz, sondern verwenden die Relativsatzparaphrase, ohne vorschnell völlige Aquivalenz zu behaupten, als "diagnostisches" Verfahren 24 zur Explikation der zuordnung ausschlieblich zum übergeordneten Bezugsnomen im Unterschied zu Bezug (gegebenenfalls gleichzeitig) auf das Prädikat oder das Satzganze. Diese Paraphrase sagt nichts aus uiber den Gegensatz restriktiv : nichtrestriktiv oder den hinsichtlich untergeordneter Prädikativität : Nichtprädikativität der Ausgangsstruktur. Sie beweist auch nicht, daB in jedem Fall nur attributive Interpretation möglich ist. Die Ersetzung durch Relativsatz veranschaulicht lediglich, daß gemäß Sprecherintention die Beziehung nur auf die ubergeordnete Nominalphrase als funktionstypkonstituierend gewertet wird, nicht grundsätzlich, daß bei Gebrauch des Partizips andere Beziehungen ausgeschlossen werden müssen.

Dies betrifft nicht nur nichtrestriktive Partizipien. So finden sich z.B. im Aksl. oder dem Material aus MS Belege, für die die Entscheidung hinsichtlich restriktiver Interpretation im Rahmen des subjektskomplexes oder zuordnung zum Prädikatskomplex mit impliziter konditionaler semantik schwierig bis unmöglich ist; ${ }^{25}$ die Ersetzung durch Relativsatz schafft hier Eindeutigkeit.

Plausibel ist die Relativsatzparaphrase nicht nur aufgrund von Ubersetzungsvergleichen und Aquivalenz im typologischen Vergleich verschiedener Sprachen: Heine z.B. vermutet Abneigung des klassischen Latein ${ }^{26}$ gegen Partizipien, bei denen keinerlei

24 Zum Deutschen: Rath 71, S. 131ff, Filipović 77, S. IIff, Bungarten 76, S. 81ff: zum Russischen: Babby 75, Kade 68, Schaller 71: zum Polnischen Weiss 77, S. $84 \mathrm{ff}$

25 Zum Deutschen: Meyer 75, S. 62, Anm. 59, Seiler 60, S. 37; zum Lateinischen: Heine 72, S. 221; vgl. M., S. 44ff, insb. S. $49 \mathrm{ff}$ und die dort angefuhrte Literatur; zum Aksl: Rủziďka, SS, S. 69; zum Polnischen Weiss 77, S. 383

$26 \mathrm{Vgl}$. Heine 72 , S. 222ff, 234, 238 
semantischer Bezug auf das Prädikat zu assoziieren ist; in solchen Fällen scheint -anders als in der silbernen Latinität oder im mittelalterlichen Mönchslatein-Relativsatz eindeutig bevorzugt $z u$ werden, obwohl Partizip "theoretisch" möglich ist.

Für das Skr. verweist Musić 35 ungekehrt auf den Gebrauch des Relativsatzes in Fällen, wo attributives Adjektiv ambig sein könnte hinsichtlich der Zugehörigkeit zum Prädikatskomplex, um die attributive Interpretation sicherzustellen, was im Aksl. ja durch die Langform des Partizips gewährleistet ist, während die Kurzform attributiv vorwiegend restriktiv oder formelhaft auftritt.

Das Russische übernimmt die (umgewertete) Korrelation und damit auch den häufigen Gebrauch des jetzt isolierten merkmallosen Partizips nach dem Bezugsnomen, doch auch innerhalb des Aksl. und des Russischen ist die grundsätzliche Äquivalenz von Partizip und Relativsatz vielfach belegt. 27 Auch Koordinierung beider Konstruktionen tritt auf:

Tut byli, nakonec, ljudi, kak budto sostavljavsiedare tretij osobennyj sloj i kotorye ne prinadlezali ..k.... no kotorych ... mozno bylo inogda vstretit'... (Rủzicka 63, Typ., S.842) Eine neuere Untersuchung der Bedingungen der Konkurrenz zwischen attributivem Partizip und Relativsatz scheint dabei insbesondere für das Russische noch $z u$ fehlen. ${ }^{28}$ Grundsätzlich ist der Anwendungsbereich des Relativsatzes größer als der der isolierten Partizipialkonstruktion.

In unserem Zusammenhang sind nur die Fälle mit Subjektsbezug von Interesse: z.B.:

stroiteli, sooruzavile krupnuju gidroelektrostanciju, projavili

27 Zur Aquivalenz von Partizip und Relativsatz in freier oder attr. Stellung im Aksl. vgl. z.B. Rúzicka 62, Zur Rolle, S. 188, Vecerka 61, S. $32 \mathrm{ff}$, ders. 61 in Sbornik, Ruzicka, SS, S. 290,320

28 Zum Aksl: Pešikan 59, S. 89ff, Večrka 61 in Sbornik: zum Russ: Rúzicka 66, S. 27ff, Kornilov 67 und die dort angefürte Literatur. Frančk 52, Buravceva 34, S. $24 f f$; vgl. hierzu auch Kovtunova 55, S. 118 
bol'soj trudovoj geroizm

Stroiteli, kotorye sooruzali krupnuju gidroelektrostanciju, projavili bol'soj trudovoj geroizm

(Ananič, S. 32)

Aus der Paraphrasebeziehung zwischen attributivem Partizip und Relativsatz folgt entgegen z.B. Babby 75 noch nicht notwendigerweise, daß der Relativsatz im Sinne einer Reihe von Ableitungsschritten dem attributiven Partizip zugrundeliegt, dieses also aus dem Relativsatz durch Reduktion abzuleiten wäre. Der in unserem Zusammenhang entscheidende Nachteil dieser Darstellung besteht darin, daß der für die Beschreibung untergeordnet prädikativer Partizipial- und Gerundialstrukturen grundlegende funktionale Unterschied zwischen restriktiven und nichtrestriktiven Strukturen aufgrund der Neutralisierung dieses Gegensatzes in der im Grunde oberflächensyntaktischen Repräsentation des Satzgliedes Attribut durch Relativsatz verwischt wird, jedenfalls, soweit man nicht auch die Intonation einbezieht.

Weiss 77 zeigt, daß die Argumente für die Attributreduktion 29 genaugenommen nur Argumente dagegen sind, Relativsätze von den weniger expliziten Attributen, z.B. auch in der Form von Partizipien abzuleiten. Gegen die Reduktionshypothese aber spricht. daß Wiederauffindbarkeit nicht in allen Fällen gesichert ist und insbesondere, daß nicht für alle Attribute eine reguläre Relativsatzparaphrase möglich ist. Dies müßte im einzelnen für das Russische noch untersucht werden, scheint aber im ganzen auch für unseren Zusammenhang plausibel. Dem entspricht Ja auch schon die alternative Ableitung von Relativsatz oder Attribut/Apposition bei Kr̈ižková ${ }^{30}$. Auch Weiss 77 legt die alternative Ableitung ${ }^{31}$ von Relativsatz und reduziertem Attribut von einem gemeinsamen Ausgangspunkt zugrunde, unterscheidet aber im Gegensatz zu Kłizková schon für die Ausgangsstruktur zwischen restriktiven und nichtrestriktiven strukturen. 32 Die alternative Ableitung bei der Beschreibung der synchronen

$29 \mathrm{Vgl}$. Weiss77, S. 85ff, $228 \mathrm{ff}$

$30 \mathrm{Vgl}$. Křizková 68, S. 35, 37ff; dies. 69, PO, S. 8

$31 \mathrm{Vgl}$. Weiss 77 , S. $185 \mathrm{ff}, 234 \mathrm{ff}$

32 a.a.o. S. $247 \mathrm{ff}, 356 \mathrm{ff}$ 
Situation entspricht zugleich dem Sachverhalt der historischen Entwicklung, daß ja die isolierten Wendungen nicht $z u$ erklären sind durch "Verkürzung" früher einmal vorhandener expliziter relativer oder adverbialer Nebensätze. 33

6.4. Zum sprachlichen Material

Attributive Interpretation kommt in unserem Material für folgende Syntagmen in Frage:

$$
\mathrm{P}+\mathrm{N} / \mathrm{N}+\mathrm{P} / \mathrm{N}+\mathrm{I}+\mathrm{P} \text {; }
$$

außerdem wird hier noch angefüht Adjektivierung und Substantivierung bzw. freie stellung von Partizipien: - P -.

Am Material ist die Distribution dieser Syntagmen festzustellen, ihre Funktion hinsichtlich restriktiver : nichtrestriktiver Beziehung $2 \mathrm{u} N$ und mögliche Ambiguitäten hinsichtlich der Zugehörigkeit zum Subjekts-bzw. Prädikatskomplex.

Während für das Russische, das hier im Vordergrund stehen soll, nur mit dem Partizip zu rechnen ist, besteht im Aksl. ${ }^{34}$ und dem in Monumenta Serbica ${ }^{35}$ untersuchten Material die Möglichkeit, daß neben dem nichtprädikativen Partizip in der Langform auch die merkmallose Kurzform verwendet wird:

$$
\begin{aligned}
& P_{1}: P_{k} \\
& \text { I kennzeichnet "Isolierung". }
\end{aligned}
$$

$$
\text { 6.4.1. } \quad P_{(1)}+N
$$

Der stellungstyp $\mathrm{P}+\mathrm{N}$ ist der Prototyp der attributiven Funktion des russischen Partizips bzw. der aksl. Langform des Partizips.

33 Vgl. Georgieva 68, S. 96ff; Rudnev 68, S. 122; Rưziłka, SS, S. $22 \mathrm{ff}$

34 zitiert nach Rúzicka, SS

35 zitiert als "MS", vgl. im Literaturverzeichnis unter Miklosich, F. (ed.) 
Wortstellung, Nichtisolierung und kongruierende Form signalisieren für das Russische eindeutig Nichtprädikativität. 36

Das Syntagma tritt auf im Wortstellungstyp $\mathrm{P}+\mathrm{N}+\mathrm{V}$ :

Mjagkij, preduprezdajuzzij zvonok velosipeda za spinoj zastavil ee sojti s tropinki

(Gnevko, S. 87)

Prikrasy, oblagoralivajułtij oreol, okotorych ja govoril, éto delo dusi

(F, S. 182)

Gotovjąčiesja k poletam kursanty uvideli ego (Adamec II 75, S.29) Moja rodiviajaja $2 a$ granicej mat' plocho govorit po-anglijaki (Babby 75, S. 2)

Sobraviesja ljudi stali znakomit'sja (Adamec II 75, S. 29) In dem Material bei Gö. Jacobsson $69^{37}$ bevorzugt relativ die Präsensform Stellung vor dem Bezugsnomen, die präteritale Form Nachstellung. Bei Voranstellung uberwiegen die nichterweiterten Belege, selbstverständlich sind jedoch auch prät. erweiterte Partizipien in Stellung vor $\mathrm{N}$ möglich.

Vgl.:

Kurivsie v koridore komandiry zamerli (Adamec II 75, S. 7) Das Partizip tritt also auf mit und ohne Erweiterung; das Syntagma $\mathrm{P}+\mathrm{N}$ kann in unterschiedlicher Wortstellung im Satz auftreten und in nichtnominativischen Kasus. Die attributive Funktion des Partizips ist nicht abhängig von der syntaktischen

Funktion von $\mathrm{N}$; vgl. 2.B. mit $\mathrm{N}$ im c.o.:

Dasa podosla k sidjastemu na kojke muziku (Adamec II 75, S. 30) Die Funktion des Partizips erschöpft sich syntaktisch und semantisch innerhalb des syntagmas $P+N$, das als ganzes permutiert werden kann, ohne daB sich die semantische struktur des Syntagmas änderte oder eine Beziehung zum Prädikat hergestellt wuirde. Damit ist nicht ausgeschlossen, daB hinsichtlich des außersprachlich Bezeichneten ein semantischer zusammenhang besteht zwischen dem nichtprädikativen, dem subjekt untergeordneten Partizip und der Prädikatshandlung, er ist jedoch nicht relevant für die

36 Zum attributiven Partizip im Russ. vgl. z.B. Isacenko 75, S. $334 \mathrm{ff}$, Mulisch 75, S. 193ff, Adamec II 75, S. $29 \mathrm{ff}$

$37 \mathrm{Vgl}$. Gö. Jacobsson 69, S. 55ff, $141 \mathrm{ff}$ 
Konstituierung des Funktionstyps. 38

Vgl. mit $\mathrm{V}+\mathrm{N}+\mathrm{P}$ mit $\mathrm{N}=$ Subj.:

Za ego spinoj poslysalsja pokazavijisja emu znakomym golos

(Adamec II 75, S. 30)

Na ékrane pokazalsjagibnuscijkorabl' (Morison, S. 52)

$=$ pokazalsjakorabl', kotoryj gib

Na ee gubach ne tajala zamerz \$aja ulybka (Gabka, S. 209)

Gegenüber $\mathrm{P}+\mathrm{N}+\mathrm{V}$ besteht hier, jedenfalls solange nicht die Intonation als Kriterium herangezogen wird, die Möglichkeit der Ambiguität hinsichtlich der zugehörigkeit des Partizips zum attributiven Syntagma $\mathrm{P}+\mathrm{N}$ :

Podchodit kakby nicego ne ponimajuscij Pavlov =

Pavlov podchodit, (kak) budto nicego ne ponimaet (Morison, S.55)

Hier liegt, nach Aufhebung der Inversion, zugrunde $N+V+P$ (s.u.). Explikation der Zuordnung zum Syntagma $P+N$ und damit $z u$ NP erfolgt durch Relativsatzparaphrase.

Dabei ist in beiden Fällen, sowohl bei Voranstellung von $\mathrm{P}+\mathrm{N}$ wie bei Nachstellung gegenüber $v$, Restriktivität und Nichtrestriktivität möglich.

Vgl.eindeutig restriktiv bei Inversion infolge Fragesatz:

Ija -dadut ze ljubjaście roditeli takoe imja doceri? (Morison,s.61)

Im Aksl.ist in der Position vor dem Bezugsnomen $(P+N)$

der Gegensatz zwischen merkmalloser Kurzform und merkmalhaft nichtprädikativer Langform maximal ausgeprägt: 39

$\mathrm{P}_{1}+\mathrm{N}$ ist eindeutig attributiv, in $\mathrm{P}_{k}+\mathrm{N}+\mathrm{V}$ ist die potentiell ambige Kurzform eindeutig nicht der Nominalphrase als Attribut zugeordnet, sondern untergeordnet prädikativ und bildet zusammen mit $\mathrm{N}$ ein "isoliertes peripherprädikatives Syntagma", 40 das als Ganzes mit dem satzschließenden Prädikat zum Satz ver-

$33 \mathrm{Vgl}$. Rudnev 63, S. 162; vgl. 2.B. auch Vondrák 28, S. 401ff, wonach allgemein die Langformen des Partizips in $\mathrm{P}+\mathrm{N}$ fast adjektivisch sind, eine bleibende Eigenschaft nennen und keine Beziehung zum Prädikat haben.

39 Vgl. M., S. 60ff; Rủzicka, SS, S. 323, 339, 363: "In attributiver ... Stellung ist das nichtprädikative Partizip vom Kurzpartizip hinsichtlich der syntaktischen Funktion absolut und sicher abgrenzbar."

40 a.a.o. S. 21 
bunden ist (s.u.).

Vgl. $2 u P_{1}+N$ :

rkoze posola me zivei óct.

J 6,57 (SS, S.340)

Das feste Epitheton steht vor dem Substantiv (vgl. demgegenüber: óco ze zivęi i sẹi vsmone. tb trorits délo J 14,10 (SS,S.339)) kbde estb rozdoi se črbijudeisks Mt 2,2 (SS,S.340) eko azb o sebe ne glaachs. no posslavii me óco, trmone zapovedu dastr J 12,49 (SS, S. 324)

Auch in $M S$ tritt $P$ in $P_{1}+N$ nur attributiv auf, während attributives $P_{k}+N$ nicht belegt ist.

Vgl. z.B.:

... dobytoka ...., jeliko suede togda nastojeztaabratija vo monastyri...

(MS..S.79)

\subsubsection{Adjektivierung}

Indiz für die syntaktische und semantische Geschlossenheit des Syntagmas $\mathrm{P}+\mathrm{N}$ ist die Tatsache, daß dieser stellungstyp Ausgangspunkt für Adjektivierung und Substantivierung von $P$ (bei Tilgung von $\mathrm{N}$ ) ist.

Zur Adjektivierung vgl. z.B.: 41

... kak nazyvali ich esli ne luzsie, to vpolne sueduscie ceniteli

$(F, S .186)$

Vot, brat, nastojascij celovek!

(Morison, S.62)

sledujustij avtobus idet bez cetverti cas (Morison, S.65)

$v$ konce koncov vyjasnilis' prjamo oselomljajustie fakty

(PaffenII,S.359 mit Hinweis auf Synoymie mit oselomitel'nyj, uzasnyj)

Die Forschungslage zum Problem der Adjektivierung von polnischen Partizipien faßt in letzter Zeit Weiss 77, S. $263 f f$ zusammen; für das Russische vgl. z.B. Gö. Jacobsson 69, S. 26ff, Mulisch 75, S. $195 \mathrm{ff} .^{42}$ Ubereinstimmung besteht hinsichtlich

41 Zum Russ. vgl. Kalakuckaja 71, Kovaliv 57, Isąenko 75,5.337ff

42 Als Kriterien für Adjektivierung nennt Mulisch 75, S.195ff u.a. - Bedeutungsdifferenzierung, vgl. z.B. opustiviiesja u nego ruki : opustivłij telovek, - Widerspruch zu den Bedingungen... 
des Verlustes der verbalen Kategorien, insbesondere der Aspektund Tempusrelationen gegenüber dem Prädikat. ${ }^{43}$ Dies durfte auch der Hintergrund sein für die außersprachlich begrüdete und im Einzelfall schwer zu objektivierende Unterscheidung von Angabe einer dauernden : momentanen Eigenschaft bzw. Handlungsgegenüber Zustandsbedeutung. 44

Jacobsson 69 und Weiss 77 z.B. zeigen anschaulich die Schwierigkeiten einer konsequenten Anwendung der derivationalen und funktionalen Kriterien. Im Ganzen ist die Grenzlinie fließend und es muß mit Fällen gerechnet werden, in denen dieselbe Form adjektiviert gebraucht wird und als Partizip fungiert.

Vgl. Jacobsson, Gö. 69, S. 27:

"In our case, it is not practical to presuppose sharp lines between the various categories, as the participles, in particular, would have a lot of borderline case."

Vgl. Weiss 77, s. 271:

"Zusammenfassend läßt sich festhalten, daß die kategoriale Loslösung eines Partizips vom zugehörigen Verb, gemeinhin als "Adjektivierung" bezeichnet, von ganz verschiedenen Faktoren bestimmt sein kann, die sich z.T. wechselseitig bedingen. ... Dabei sind jedoch verschiedene Grade von Adjektiviertheit feststellbar, je nach Anzahl (und Art?) der beteiligten Kriterien; der Versuch einer Klassifizierung des Materials nach solchen Adjektiviertheitsstufen ist freilich noch nie unternommen worden."

Gerade die typischen derivationalen Merkmale der Adjektivierung sind für die polnischen Partizipien kein geeignetes Charakteristikum ihrer kategorialen Verselbständigung. 45

Tendenziell kennzeichnend für Adjektivierung von russischen Partizipien sind distributionell u.a. Stellung vor dem Bezugsnomen, Präsensform (die ja Voranstellung bevorzugt) und Nicht-

42 ... für die Bildung des Partizips, - Existenz von Formen, zu denen kein entsprechendes Verb vorhanden ist, - Gebrauch in festen Wendungen

43 Kovaliv 57, S. 14; Gvozdev I 73, S. 379; Mulisch 75, S. 195ff; Weiss 77, S. 265ff, 271; Isacenko 75, S. $334 \mathrm{ff}$

$44 \mathrm{Vgl}$. z.B. Vondrák 28, S. 401ff; Mulisch 75, S. 194 ; vgl. kritisch Jacobsson, Gö. 69, S. 56 zu Bicilli

$45 \mathrm{Vgl}$. Weiss 77 , S. $263 \mathrm{ff}$ 
erweiterung durch Adverb oder "Complement". 46

Auf die Relevanz der Nichterweiterung isolierter Partizipialstrukturen für die Tendenz zur Adjektivierung verweist Francuk 52. Demnach wird in solchen Fällen Adjektivierung im wesentlichen dann verhindert, wenn entweder ein konkurrierendes Adjektiv vorliegt (z.B. namoksij : mokryj, pritichšij : tichij) oder wenn für das alleinstehende Partizip Bezug nicht nur auf das Subjekt, sondern auch auf das Prädikat vorliegt.

Eben dies, das Fehlen des Bezuges auf das Prädikat, gilt am deutlichsten für den stellungstyp $\mathrm{P}+\mathrm{N}$ bei Restriktivität des Partizips.

Vgl. 2. B.:

...čto nadležašij moment uže upušcen (F, S.178)

èpitety, kotorye ja staralsja k nemu prilozit'.. ne sovsem podchodjašcie épitety

(F,S.27)

$\mathrm{Zu}$ beachten ist, daß heute die genuin russische Form auf - $c$ eindeutig Adjektive kennzeichnet gegenüber der ihrer Herkunft nach aksl. Form auf - క̌̌-; z.T. treten Parallelen von Adjektiven

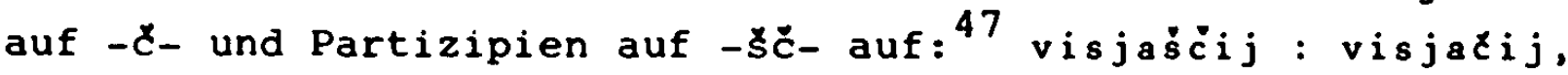
gorjaščij: gorjačij, żgušcij : žgučij u.a.m.

Auch für die folgenden skr. Beispiele für Adjektivierung des Partizips kann als Ausgangspunkt restriktive Bedeutung unterstellt werden:

vladajući sistem, idući put, bivß̌i ministar, 'vladajuća klasa, leteća turdava, iduća sedmica, viseći vrtovi

Vgl.:

Biv\&a Jugoslavija bila je agrarna drăava (Brabec,s.260)

Dabei ist im einzelnen nicht immer exakt $z u$ unterscheiden zwischen restriktivem Gebrauch des (adjektivierten) Partizips, das auch bei einem anderen Nomen auftritt, und formelhaften Wendungen, in denen das Partizip mehr oder weniger schmückendes Beiwort bei der Bezeichnung eines feststehenden Begriffes ist. Im Ergebnis liegt in beiden Fällen identifizierende Funk-

$46 \mathrm{Vgl}$. Jacobsson, Gö. 69, S. 28ff, 57, 118ff, 175; Francuk 52, S. $6 \mathrm{ff}$

47 Vgl. z.B. die Beispiele bei Morison 59, s. 68 
tion vor.

In dem Material aus $M S$ ist bei der Distribution $P_{1}+N^{48}$ die Tendenz zur Formelhaftigkeit deutlich; nichterweiterte Beispiele uberwiegen und werden als Beispiel für Adjektivierung gewertet: vgl. z.B.:

kralevbstrujei (MS,S.75), gospodrstrujustii (MS,S.81), vsenastojesteje (MS,S.163,165,159), vsenastoestee (MS,S.145), byvsii (MS, S. 86), pogibsie (MS,S.220), pocivs̉i (MS,S.182)

... pogibšsie ōvoce domu izravileva ka istinnomu pastiru i bogu vsedrbitelju vaznvastene bise.. (MS, S. 220)

Für die erweiterten Belege, bei denen die Entscheidung über Adjektivierung offen bleiben mag, liegt u.E. restriktive Interpretation nahe; vgl. z.B.

chotei (MS,S.80), nastojesti (MS,S.38), nastojestaa (MS,S.79), vgl.:... i dobytoka zivoga i mrntvoga, jeliko suede togda nastojestaa bratija vn monastyri... (MS,S.79)

Gleichzeitig veranschaulichen die oben angefuhrten restriktiven/adjektivierten Belege für $\mathrm{P}_{1}+\mathrm{N}$ die Affinität dieses Typs zur Substantivierung:

aste i tow samn gospodnstvujustii kraal- srobsnske zemlje

(MS, S.81)

da ouzima jepiskoupija jednnvoln, a drougi da ouzima gospodnstvoujusti

... ni knezn ne sévaston ni vladuštii vzemli.. (MS,S.138)

\subsubsection{Freie Stellung / Substantivierung}

Der stellungstyp $\mathrm{P}+\mathrm{N}$ bzw. fur das Aksl. und $M S \mathrm{P}_{1}+\mathrm{N}$ ist Ausgangspunkt auch für die Substantivierung; diese kann als oberflächensyntaktisches Ergebnis einer restriktiven Attribuierung zu einem sprachlich nicht realisierten Attributkern gewertet werden. 49 Das Ergebnis ist die sogenannte freie Stellung des

48 M., S. 62ff; vgl. auch die Beispiele a.a.0. S. 10

49 Zum Aksl. vgl. Rưzicka, SS, S. 289ff, 341, 362, Vełerka 61, S. 161ff, M., S. 62ff; z.Poln. Weiss 77,s.207ff,377ff,393 
Partizips, d.h. Nichtprädikativität und Nichtunterordnung unter ein Bezugsnomen. Vielmehr verhält sich das Partizip distributionell selbst wie ein Nomen. Eine exakte Definition allerdings ist ähnlich wie bei der Adjektivierung schwierig. Weiss 77 unterscheidet für polnische Partizipialkonstruktionen zwischen jenen Fällen, die syntaktisch ableitbar sind (okkasionelle substantivierung) und jenen, die als fertige Lexikoneinheiten in den "phrase marker" einzusetzen sind (generelle Substantivierung). Kriterien für letztere sind z.B. Fehlen eines entsprechenden Verbums, verändertes Valenzverhalten, Bedeutungsverengung, Gebrauch des prädikativen Instrumentals bei Kopula sowie Besonderheiten hinsichtlich Aspekt und Genus, die hier nicht erörtert werden sollen. 50

Für das Russische stellt sich die Problematik grundsätzlich analog. 51 Als hauptsächliches Kriterium neben dem Fehlen des Beziehungswortes wird wieder das Fehlen aspektual-temporaler Relationen zum Prädikat angefüht.

Vgl. z.B.:

Ved'viskusstve nacinajušij pocti vsegda dolzen obrašcat'sja... (F, S. 185)

Mulisch 75 unterscheidet "verbale substantivierung", wenn das alleinstehende Partizip erweitert ist, "okkasionelle substantivierung" bei Partizip ohne Erweiterung, das seinerseits auch in Unterordnung auftritt (U.E. wären derartige Fälle eher als Adjektivierung anzusprechen, während Mulisch hier partizipialen Gebrauch annimmt) und "usuelle Substantivierung" bei Gebrauch nur noch als Substantiv und völligem Verlust des Kontaktes zum Verbalparadigma.

Vgl. 2.B.:

Javivichsja na konferenciju prosjat zaregistrirovat'sja Večnaja slava pavł̌im aber: Velnaja slava pavisim gerojam

$50 \mathrm{Vgl}$. Weiss 77, S. 208ff, $213 f f$

51 Zum Russ. vgl. 2.B. die Beispiele bei Leitfaden 71, S. 108, Paffen II, S. 349, 359, Mulisch 75, S. 196ff, Jacobsson, Gö. 69 , S. $81 \mathrm{ff}$ 


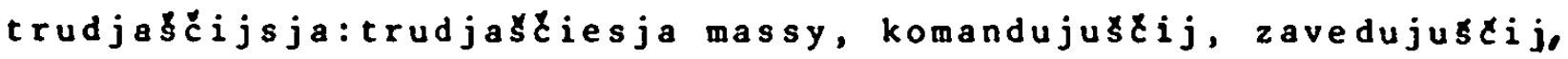
minuvsee, posledujušcee, budustee, byvsee, mlekopitajustee, $s$ umaš ed š $\mathrm{i} j$

Z.B. bei sluzašij ist die Substantivierung auch durch den abweichenden Akzent signalisiert.

Im einzelnen wären hier noch ausführliche Untersuchungen $z u$ den Bedingungen der Bildung und des Gebrauchs departizipialer Substantive, auch im typologischen vergleich, 52 nötig. Für unsere Zwecke soll der Hinweis auf diese wenigen Beispiele genügen.

Im Aksl. unterscheidet sich die "freie stellung" der Langform des Partizips 53 vom Substantiv dadurch, daß bei Partizip Erweiterung durch Akkusativobjekt möglich ist und daß (ähnlich wie im Russischen, jedenfalls bei okkasioneller Substantivierung) 54 das Partizip in der Regel nicht durch adjektivisches oder partizipielles Attribut ergänzt wird. Das Partizip fungiert als Kern einer Nominalphrase. Es besteht positionelle Aquivalenz mit den substantivischen Pronomina und mit Relativsyntagmen des Typs /ize + Verb (3.Pers.sing. oder Pl).

Vgl. z.B.:

i aste ljubite ljubesteje vy

L 6,32 (SS, S. 289)

séjęi slovo séetr

Mr 4.14 (SS, S.292)

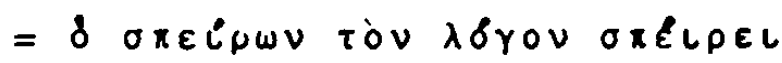

Ganz auszuschließen ist die substantivische Geltung (= freie Stellung) auch für die merkmallose Kurzform ${ }^{55}$ des Partizips nicht, wobei wiederum die restriktive Bedeutung deutlich wird. Vgl.:

cre jezykomb. ustojetrimin. i obladajoste imi blagodétele naricajotbse.

L 22, 25 (SS, S. 235)

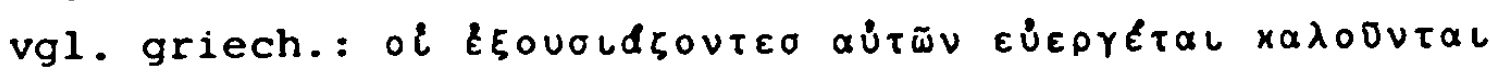
lat.: qui potestatem habent

togda soste vrijujei da bégajotb vr gory (Var.: sqstei)

L 21,21 (SS, S. 326)

$52 \mathrm{Vgl}$. z.B. Weiss 77 , S. $214 \mathrm{ff}$

53 Vgl.Rúzicka, SS, S. 274

54 anders Weiss 77, S. 209 zum Polnischen 


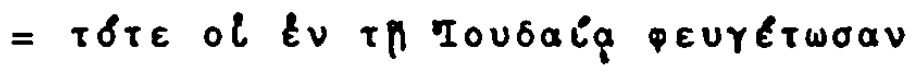

Eine exakte Definition des Gegensatzes alleinstehendes Partizip : Ubergang in die Wortart Substantiv bleibt problematisch (s.o.).

Vgl. als Beispiele für freie stellung in $\mathrm{MS}^{56}$ :

ljubei me vozljubleno boudeto

(MS, S. 72)

obrateto bo me, rex̌e, bojełtei se tebe i védoustei surdernia

tvoja

(MS, S.137)

iftourti slovesa blaga zizno vexnuju obreftouto (MS, S.110)

Vgl. als Beispiel für Substantivierung von Formen, für die bei Unterordnung unter Nomen Adjektivierung angenommen worden war: ...sam gospodostvujustii kralb...

(MS, S.81)

gegenüber:

... ni knezo ne sévasto ni vladư̆tii voremle (MS,S.138)

Vgl. analog:

vladustii (MS,S.135), gospodostroujusti (MS,S.15), kralevbstroujei (MS,S.75) u.ä.

Freie Stellung der Kurzform ist, analog zum Aksl., ungleich seltener belegt. 57

6.4.2. $N+I+P$

Die transformationelle Verwandtschaft des attributiven Syntagmas $\mathrm{P}+\mathrm{N} z \mathrm{u} \mathrm{N}+\mathrm{I}+\mathrm{P}$ (Nachstellung mit Isolierung) 58 veranschaulichen für das Russische Beispiele wie gotovjastiesja $k$ poletam kursanty uvideli ego $\rightarrow$ kursanty, gotovjastiesjak poletam, uvideli ego (Adamec II 75,S.30)

56 weitere Beispiele bei M.. S. 63

$57 \mathrm{Vgl}$. M., S. 61

$58 \mathrm{Vgl}$. zur Permutationstransformation auch z.B. Kŕižková 68 , S. 37; zum Deutschen vgl. Bungarten 76, S. 82ff:"Eine Unterstitzung, aufbauend auf klare Forderungen mit entsprechendem Nachdruck, hätte bereits am Tage der Entfihrung einsetzen müssen $\rightarrow$ Eine auf klare Forderungen mit entsprechendem Nachdruck aufbauende Unterstutzung hätte bereits am Tage der Entführung einsetzen müssen"; vgl. hierzu a.a.o. S. $83 f f$ zur Herstellung des Endkontakts zum Bezugsnomen und Ausstattung des Partizips mit Bezugsmorphemen. 
Snjavtiesja s jakora melkie suda uchodili v dozor $\rightarrow$ melkie suda, snjavsiesja s jakora, uchodili v dozor (Ananǐ,s.32)

Vgl. auch in MS:

jako da chotei staroco prébyvati ... da se ne stavi óto inoude (MS, S. 80)

i öto inoude starncn chotei prébyvati... da se ne stavii..

(MS, S. 75)

Statistisch ergibt sich aus dem Material bei Jacobsson, Gö. 69. daß Part. präs. akt. Stellung vor dem Bezugsnomen bevorzugt, Part. prät. akt. Nachstellung mit Isolierung. Absolut überwiegt auch bei Postposition Part. präs. akt. ${ }^{59}$ In Voranstellung ohne Isolierung treten Partizipien mit und ohne Erweiterung auf, in isolierter Postposition so gut wie ausschließlich erweiterte Partizipialstrukturen. ${ }^{60}$ Erweiterung ist eine wesentliche, nicht aber zwingende Motivierung für Postposition mit Isolierung. Nichterweiterung bei Postposition und Isolierung ist in unserem Material für akt. Partizipien bisher nicht belegt, aber nicht auszuschließen.

Vgl. mit koordinierten Adjektiven:

Jasnoe $i$ tocnoe ob-jasnenie ubedilo nas $\rightarrow$ ob-jasnenie, jasnoe i tocnoe, ubedilo nas (Leitf., S.151)

Solnce, krasnoe i jarkoe, igralo so mnoj (Adamec II 75,S.182) Vgl. $z u$ den distributionellen Bedingungen der Postposition Jacobsson, Gö. 69, S. 143 :

"a) Participles without a complement normally stand before the correlate

b) For the participial categories, represented above, it is normal for them to stand after their correlates when they have an accusative object as their complements

c) When the complement to the $\breve{s} \check{c}_{j}+$ form is the genitive, the participle may stand either before or after its correlate"

Grundsätzlich hat die Postposition mit Isolierung die Funktion

$59 \mathrm{Vgl}$. Jacobsson, Gö. 69, S. $41 \mathrm{ff}, 43 \mathrm{ff}, 55 \mathrm{ff}$

$60 \mathrm{Vgl}$. Adamec II 75, S. 30 zur Postposition mit Isolierung: "Dannaja transformacija, odnako, vozmozna ne vsegda, osobenno esli u prißastija net rasprostranitelej"; vgl. Jacobsson, Gö. 69, S. $124 \mathrm{ff}, 141 \mathrm{ff}$ 
der Hervorhebung 61 und Betonung des semantischen Eigenwerts des partizipialen Attribus. Für das Polnische nennt Weiss ${ }^{62}$ als relevant für die Postposition des Attributs neben dem Umfang der attributiven Wendung den semantischen Gegensatz zwischen aktueller (okkasioneller) und genereller (ständiger) Charakterisierung, wobei generalisierende Attribute im Polnischen Postposition bevorzugen.

6.4.2.1. N $N+I+P+I+V$

Der stark ausgebaute Gebrauch isolierter Partizipialstrukturen ist ein wesentliches Charakteristikum des Russsichen gegenüber anderen slavischen Sprachen. ${ }^{63}$ Der Distributionstyp $N+I+P+I+V$ ist dabei innerhalb des Russischen und im typologischen vergleich von erheblichem strukturellem Gewicht. 64

Vgl. nichtrestriktiv:

Losad', begusciaja po sosse, spotknulas' (Isacenko 75,S.334) Mne kakoj-tokapitan, nazyvajuszij sebja vaśim rodstrennikom, ... vse piset nepriličnye pis'ma (Morison, S.60) Vse skoro uleglis'; ja, kurjažij, sidel na brevne pered saraem i govoril s Pavlom (Morison, S.53) Danvic, nabljudavsij vsju scenu iz okna vestibjula, byl vne sebja ot obidy i zlosti (Adamec II 75, S.183) Syn ego Aleksandr, vstupivíi na prestol posle ubijstra Pavla, prinimal ucastie vagovore protiv otca = Sein Sohn Aleksander, der nach der Ermordung pauls den Thron bestiegen hate, nahm an der Verschwörung gegen seinen Vater teil (Paffen II,S.356) Ćto ... talmudist, nikogda snimavsijermolki,učenosti ... prevoschodil svoego kollegu-inoverca (F.S.3O)

$61 \mathrm{Vgl}$. Adamec II 75, S. 30; Mulisch 75, S. 193; Vecerka 61, S. 40; Rủzicka, SS, S. 364

62 Weiss 77, S. $222 \mathrm{ff}$

$63 \mathrm{Vgl.}$. B. Kornilov $67 \mathrm{mit}$ Hinweis auch auf germanische und romanische Sprachen

64 Vgl. Rażička 63, Typ., s. 837 
restriktiv:

Liş' Eelovek, umejuščij vesti sebja ticho, uvidit v okeane mnogo

(Adamec II 75, S.170)

Kompozitor, presytiv\$ijsja orkestrovym impressionizmom i potomu ne obucajuścijsja instrumentovke, podoben vraču, kotoryj... $(F, S .185)$

Cenzor, davijij razresenie na petatanie raboty Lomonosova, by uvolen so sluzby i polueil strogij vygovor (Adamec II 75,S.181) Durch den Distributionstyp $\mathrm{N}+\mathrm{I}+\mathrm{P}+\mathrm{I}+\mathrm{V}$ mit attributivem und bei Nichtrestriktivität untergeordnet prädikativem Partizip ist für das Russische die strukturelle Möglichkeit zur untergeordneten Prädikativität des merkmallosen Partizips nicht nur im Rahmen des Nominalkomplexes mit fortbestehender einseitiger Unterordnung unter $\mathrm{N}^{65}$, sondern auch bei $\mathrm{P}+\mathrm{I}+\mathrm{N}+\mathrm{V}$ in "sekundärprädikativer" Funktion, d.h. außerhalb des attributiven Syntagmas, vorgezeichnet. Indiz hierfür ist die nicht auszuschließende Ambiguität des nichtrestriktiven untergeordnet prädikativen Partizips in Zwischenstellung hinsichtlich der Zuordnung zum Subjekts- oder Prädikatskomplex (s.u.). Gegenüber der ausgeprägten Neigung des Russischen zur partizipialen oder gerundialen Kondensierung auf der Grundlage der Korrelation ist in anderen Slavinen die Tendenz zur Bevorzugung expliziter hypotaktischer Formen der Prädikation zu beobachten.

Statt des isolierten Partizips nach $\mathrm{N}$ bevorzugen $\mathrm{z} . \mathrm{B}$. das Ukr. und WeiBr. Relativsatz ${ }^{66}$. Im Skr. ${ }^{67}$ wurden Partizipien entweder adjektiviert oder durch Relativsatz ersetzt, so daß eine opposition Partizip : Gerund nicht mehr bestand und das Gerund in sekundärprädikativer Funktion übrigblieb (s.o.).

Auch im Slk. besteht die Tendenz, russische attributive Partizipien $z$ ersetzen durch Relativsatz (oder durch Part. pass.): ${ }^{68}$

$65 \mathrm{Vgl}$. Weiss 77 , S. 382

$66 \mathrm{Vgl}$. Bukatevic 58 (ed.), S. 314, 320

67 Vgl. Pešikan 59, S. 96; allg.zu Relativsätzen im Skr.Gallis 56

68 Svetlikk 70, S. 257: "V slovackom jazyke takie konstrukcii ne imejut sootvetstvija, tak kak posle atributivnogo pri-... 
Osobenno choroba byla sosna, sverkajulzaja sverchu donizu Slk: Velmi krásna bola oosna, Liariaca (ktorá ziarila) od vrchu az... (svetlik, S. 267)

Nauka, imejuglaja kakuju-nibud' cel' vmesto istinnogo znanija, - ne nauka

Slk: Veda, ktorá má namiesto skutoéného poznania iné ciele, nie je veda (Svetlik, S. 267)

okno, vychodjašee na ulicu, bylo zakryto Slk: okno, obrátené (ktoré viedlo) na ulicu, bolo zatvorené (Svetlik, S. 267)

Während im Russischen Gerund und Partizip in Korrelation stehen, gilt z.B. im Cech.9 nicht die Möglichkeit der (partiellen) Synonymie, sondern Transgressiv und akt. Partizip stehen in Opposition zueinander. Transgressiv und akt. Partizip werden, anders als im Russischen, nicht koordiniert. Präteritalpartizip (wie auch im Poln.) und für das Russische relevantes postpositives pf. Transgressiv fehlen weitestgehend. So ergibt sich auch im Cech. gegenuiber dem Russischen Bevorzugung und z.T. ausschließliche Möglichkeit der expliziten hypotaktischen Prädikation. Isolierung ist hier nicht in gleichem Maße wie im Russischen relevant; durchaus üblich ist die im Russischen seltene Distribution $\mathrm{N}+\mathrm{P}$, wo infolge Nichtisolierung das Partizip weniger in der Lage ist. Erweiterungen aufzunehmen. 70 Nicht. möglich ist, aufgrund des Fehlens der Korrelation, der russische sekundärprädikative Typ $\mathrm{P}+\mathrm{I}+\mathrm{N}+\mathrm{V}$.

Vgl. Cech. Relativsatz statt russ. N+I+P:

...Adrian, rodiv8ijsja v 1885 godu

Eech: ...Adrian(a), jenz se roku 1885 narodil

(Rủžička 63,Typ.,S.836)

kak s nego uze sbezalo slovo, vtaine smutivee menja

68 ... Castija ne možet vystupat' zavisimyj Łlen. V perevode na slovackij jazyk prichoditsja ispol'zovat' pridatoznye predlozenija." vgl.a.a.o. S. 267

69 Vgl. 2.B. Rủzicka 63, Typ., S. 834ff, 843ff, 850 und die dort angefühte Literatur: ders. 62, Korr.. S. 683ff; Kt1Zková 69, Synt.ch., S. 118; Sint.r.J., S. 139ff, $236 \mathrm{ff}$

70 Vgl. Rủzicka 63, Typ., S. 838ff; Źaźa 64, S. 185 
Cech: a jiz se z nêho vyłinulo slovo, ję mě potají uź uvedlo (Rúzička 63,TYp.,S.836)

èto byl zelovek, zabyvajušcij vse čech.: byl to clovêk, ktery na všecko zapomíná

(Rủzička 63,Typ.,S.837)

Im Polnischen 71 ist die situation ähnlich, da auch hier akt. Partizip und Gerund in Opposition zueinander stehen, d.h. Ersetzung nicht möglich ist, und neben $N+I+P$ Nichtisolierung, d.h. $N+P$ häufig ist. Demgemäß fehlt auch der Typ $P+I+N+V$, der für die Definition der russischen Korrelation mitentscheidend ist. Allerdings scheint die Koordinierung des akt. Partizips mit anderen kongruierenden sekundärprädikativen strukturen möglich zu sein; vgl.:

Z natury zagodny i nie szukajacy konfliktów, tym razem jednak nie mogłem sie opanować

(Weiss 77, s.380)

In Zwischenstellung, für die wir im Russischen bei akt. Partizip Ambiguität hinsichtlich der Zugehörigkeit zu NP : VP nicht von vorneherein ausschließen, ist im Polnischen der Gegensatz zwischen akt. Partizip und Gerund eindeutig distinktiv: Charlotte Corday, stojac / stojaca na chwiejnych nogach, rozpoczynaza monolog

(Weiss 77, S.359)

In unserem Zusammenhang soll nicht der typologische vergleich, sondern die Unterscheidung von Funktionstypen im Russischen im Vordergrund stehen.

An historischen Einflüssen zur Erklärung der Sonderstellung des Russischen hinsichtlich der ausgebauten untergeoraneten Prädikativität nennt Rủickka einerseits die aksl. Tradition und andererseits die Interferenz des Russischen mit dem Französischen, für das ebenfalls die Isolierung ein wesentliches strukturelement ist. 72

Anders als im Deutschen ${ }^{73}$, Eech. ${ }^{74}$ oder Poln. ${ }^{75}$ ist im Russi-

$71 \mathrm{Vgl}$. Weiss $77, \mathrm{~s} .354,380$

72 Vgl. Rúzicka 63, Typ.., S. 841 mit Hinweis auf Bally; zum Franz. vgl. Banaru 75

$73 \mathrm{Vgl}$. Bungarten 76, S. 136

74 Vgl. Kriźková 69,Po, S. 122; Z̉aža 64, S. 186

$75 \mathrm{Vgl}$. Weiss $77, \mathrm{~s} .88,228$ 
schen auch mit isolierten restriktiven Partizipien in der Position $N+I+P$ zu rechnen. 76 (s.o.)

Dabei legen wir als Kriterium der Isolierung die Interpunktion zugrunde. Dies bedürfte der Uberprüfung und z.T. wohl der Korrektur anhand des Vergleichs mit der gesprochenen Rede, scheint uns aber konsequenter als, wie z.B. Adamec II $75^{77}$, ad hoc zwischen "formaler" und "funktionaler" Isolierung $z u$ unterscheiden, wobei dann "funktionale" Nichtisolierung synonym mit Restriktivität gebraucht wird. Die Wahrscheinlichkeit immerhin einer Pause auch in der gesprochenen Rede düfte zunehmen bei wachsender Länge der restriktiven Wendung ${ }^{78}$, auch wenn die Möglichkeit restriktiver postpos. isolierter Partizipien in den Handbuichern gelegentlich übergangen bzw. Isolierung der Nichtrestriktivität eineindeutig zugeordnet wird. 79

Auch Rüzicka 66, S. 27 ff führt nur fakultative strukturen nach $\mathrm{N}$ an ("App"), verweist andernorts aber doch auf die Möglichkeit der Nichtprädikativität nachgestellter isolierter Partizipien (ders. 63, Typ.. S. 837).

Demgegenüber erörtert Kornilov ausführlich die Unterscheidung restriktiver und nichtrestriktiver Partizipien in $N+I+P$. Während für nichtrestriktive Strukturen eine Reihe von Paraphrasen infragekommen, die auf ihre Affinität zu untergeordnet prädikativen Strukturen im Rahmen des Prädikatskomplexes verweisen (s.o.), nennt Kornilov für restriktive isolierte Partizipialstrukturen ausschließlich die Möglichkeit der Aufhebung der Isolierung und Voranstellung (die fa grundsätzlich auch für isolierte nichtrestriktive strukturen nicht ausgeschlossen ist): vgl. z.B.:

76 Vgl. Kornilov 64; ders. 67; ders. 71, s. 96, 99ff; Francuk 52; Zaza 65, S. 70-72; ders. 64, S. 181, 183, 186ff; KH1Zková 69, PO, S. 8; dies. 68; dies.69, Synt.ch., S. 122

$77 \mathrm{Vgl}$. Adamec II 75, S. $170 \mathrm{ff}, 184$

$78 \mathrm{Vgl}$. Zaza 64, S. 183

$79 \mathrm{Vgl}$. 2.B. Ananic 72, S. 30ff: Mulisch 75, S. 193ff: Gabka 76, S. 216ff; Gvozdev II 73, S. 152ff; Panzer 75, S. 177ff: Rudnev 63, S. $158 \mathrm{ff}$; ders. 68 , S. $149 \mathrm{ff}$ 
Celovek, sidevšij protiv Zenšciny, nalil rjumku vodki i podvinul $k$ nej = Sidevsij protiv zenšiny zelovek nalil rjumku vodki i podvinul k nej

(Kornilov 71, S. 100)

6.4.2.2. $V+N+I+P$

Die Fügung $\mathrm{N}+\mathrm{I}+\mathrm{P}$ tritt nicht nur bei Zwischenstellung des Partizips, sondern auch bei Endstellung auf.

Bei Bungarten ist für deutsche isolierte Partizipialkonstruktionen der stellungstyp $\mathrm{V}+\mathrm{N}+\mathrm{I}+\mathrm{P}$ der einzige, für den er Ambiguität hinsichtlich attributiver : adverbialer Funktion annimmt, während für $\mathrm{N}+\mathrm{I}+\mathrm{P}+\mathrm{I}+\mathrm{V}$ attributive Funktion des Partizips gesichert sein soll. 80

vgl. z.B. ambig:

Und als sie wieder allein waren und er den unterbrochenen Satz zu Ende bringen wollte, da sagte sie,starr und geradeaus in den Wald hinein blickend, mit einer stimme wie trockenes Holz...

vgl.:

Da sagte sie, die starr und geradeaus in den Wald hinein blickte, mit einer stimme...

oder:

Da sagte sie, während sie starr und geradeaus in den wald hinein blickte, mit einer stimme...

Bungarten zieht die adverbiale Interpretation vor.

In dem folgenden russischen Beleg könnte Relativsatz ambig hinsichtlich des Bezugsnomens verstanden werden, Partizip ist eindeutig:

Ne bylo uze ni odnogo teloveka, kotorogo ne zachvatila voljak zizni, ischodjascaja iz ètogo koroten'kogo suscestva s uprjamoju vichrastoj golovoj

gegenuiber:

... voljak zizni, kotoraja ischodit... (Francuk 52,5.12)

Die Untersuchung von Strukturen mit $N+I+P$ am Satzende erforder-

$80 \mathrm{Vgl}$. Bungarten 76, S. $89 \mathrm{ff}, 157 \mathrm{ff}$ 
te Ụberlegungen zum Problem des Satztyps, die unser Thema überschreiten. N kann auftreten als Subjekt oder Prädikatsnomen, das Partizip kann restriktiv oder nichtrestriktiv fungieren. Vgl. hierzu auch $P_{k}$ im Aksl.: ibo azv $\overline{k k}$ esmb podo vlastely. utinent. imeje... voje

$$
\text { L } 7,8 \text { (SS, S. 233) }
$$

ucineno fungiert hier attributiv (restriktiv), imese peripherprädikativ.

Restriktiv werden die folgenden russischen Beispiele klassifiziert; als Paraphrase kommt Relativsatz infrage, nicht aber Koordinierung oder selbständiger Satz:

Eto rabolij, primenjajustij novyj metod = étoraborij, kotory j...

(Leitfaden, S. 152)

Misticeskoe... napravlenie,v kotorom,dumatsjame, porti neizbezno dvizetsja ćelovereskaja mysl', stremjastajas' postic' prirodu

(F,S.41)

Leverkjun ne pervyj i ne poslednij kompozitor, ljubivsij prjatat' v suoich trudach... cifry

(F, S. 190)

Ne uspel ja vzjat'sja za pero, kak s nego uze sbezalo slovo, vtaine smutivsee menja, slovo: "genial'nost" (F,S.26)

Isportilsja motor, davavij svet

(Paffen II,S.355)

Nichtrestriktiv (Paraphrase durch koordinierte Prädikation oder durch selbständigen Satz möglich):

Nedavno pojavilos' interesnoe soobsenie, prolivavsee novyj

svet na étu zagadoznuju problemu

(Adamec II 75, S.180)

... U Adriana nacalos' sil'noe golovokruzenie, zastavivsee ego privlec'

(F, S. 193)

Eto byl obrazec, utoncennoj muzykal'noj zivopisi, svidetel'stuujustijo porazitel'nom pristrastiik ... (F,S.186)

V rjad s nimi zanimala polsteny ogromnaja poternevsaja kartina, pisannaja masljannymi kraskami, izobrazavaja cvety, frukty

(Rudnev 63, S.160)

On celovek s bol'sim vnutrennim soderzaniem, gluboko dumajušij i cuvstrujustij = Er ist ein Mensch mit großem inneren Gehalt; er denkt und fühlt tief.

(Paffen II,S.354)

Eine exakte Beschreibung derartiger nichtrestriktiver Partizi- 
pien in Endstellung macht vorläufig noch Schwierigkeiten. 81 Jedenfalls ist die Identifizierung des Bezugsnomens hier jeweils durch andere Mittel als das akt. Partizip gesichert. Dies vorausgesetzt, wäre $z u$ fragen,ob nicht statt der üblichen klassifizierung als (nichtrestriktives) Attribut hier eher von Neutralisierung des Gegensatzes der Zugehörigkeit zu NP bzw. VP zu sprechen ist, ${ }^{82}$ wie sie $\mathrm{u} . \mathrm{E}$. auch die weitgehende Beliebigkeit der wahl zwischen verschiedenen Paraphrasen bei dieser Distribution nahelegt (Relativsatz, Koordinierung, selbständiger Satz). Wesentlich ist immerhin die Möglichkeit nichtrestriktiver Strukturen auch am Satzende; dem entspricht die Nichtfokussierung, d.h. diese Belege fungieren nicht als alleiniges Intonationszentrum des Satzes.

6.4.2.3. $N+P$

Im Russischen überwiegen bei Nachstellung des attributiven Partizips die isolierten Belege bei weitem. Es besteht Ambiguität hinsichtlich restriktiv : nichtrestriktiv. 83 Gegenüber dem Poln. oder Kech. ungleich seltener, aber eben doch, treten auch nichtisolierte Belege auf:

Vgl. mit Adjektiv in Zwischenstellung:

Celovek tverdyj ne boitsja prepijatstvij (Rudnev 68,S.151)

Adjektiv in Endstellung:

Na stole stojali belye rozy:

Na stole stojali rozy belye, na podokonnike rozy krasnye (Ktizková $68,5.37$ )

$81 \mathrm{Vgl}$. Weiss 77, S. 90,92, Anm. 106

$82 \mathrm{Zu}$ deutschen isolierten Strukturen am Satzende vgl.Sviblova 62

83 Vgl.Rủzicka 63, Typ., S. 837: "Die Isolierung der Partizipien (und Adjektive) ist in Verbindung mit der stellung vor oder nach dem Beziehungsnomen im Russ. zu einer Korrelation ausgebaut: Isolierung des präpositiven Partizips signalisiert periphere Prädikativität, Isolierung des postpositiven Partizips signalisiert diese nicht. Das tschech. Partizip weist diese Korrelation der Isolierung nicht auf.": vgl. auch Adamec II 75, S. 29ff; Kolsanskij 62, S. 36 
Mit Partizip in Zwischenstellung:

List'ja pozeltevile po vetru letjat (sint.r.j.74,s.101) in Endstellung - nach unbestimmten Pronomen:

... Cto bylo zdes' netto pochozee... i brosaviee otsuet dusevnosti na... (F,S.189)

... tro v nich byl kakoj-to misticeskij privkus, netto v bylye vremena scitavbeesja cut' $l i$ ne koldovstrom (F,S.36)

Vgl. auch:

Ja sam - Zelovek uvlekajuštijsja

(Morison, S.59)

U.E. ist bei diesen Belegen für Nachstellung und Nichtisolierung $^{84}$ Restriktivität evident. Dem entspricht Hervorhebung durch Intonation. $N$ kann als subjekt oder Prädikatsnomen fungieren, Endstellung des nichtisolierten Partizips überwiegt in unserem Material bei weitem. Nichterweiterung überwiegt. ist aber offenbar nicht Bedingung für Nichtisolierung. Während im Russischen die Nichtisolierung des nachgestellten Partizips die Ausnahme darstellt, insbesondere bei Endstellung auftritt und in unserem Material Indiz für Restriktivität ist. sind im Poln. und Cech. nichtisolierte strukturen durchaus ublich. Nichtisolierung (jedenfalls graphisch) scheint im Cech. kein Indiz für Restriktivität zu sein.

Vgl. 2.B. mit Adjektiv:

My, zdorovye, mozem dvigat'sja

Cech.: My zrdravi se múzeme pohybovat (Ruzicka 63,Typ.,s.842)

Für das Poln. legt Weiss ${ }^{85}$ zumindest nahe, daß, jedenfalls bei Stellung nach $N$, isolierte strukturen als nichtrestriktiv und nichtisolierte als restriktiv $z$ werten sind. Allerdings wären zur Absicherung dieser Hinweise zum Poln. und Kech. noch ausfürlichere Materialvergleiche nötig.

Zum Poln. vgl.:

$84 \mathrm{Vgl}$. auch Rudnev 59, S. 58; ders. 68, S. 145, 151; Morison 59, S. 59; Gnevko 75, S. 75ff; Ktizková 68, S. 37; . dies. 69, PO, S. 8; Zaža 64, S. 182

85 Die Formulierung bei Weiss 77, S. 228 ist nicht ganz eindeutig: "... ist die Abtrennung durch Pause schon fakultatives Charakteristikum einer Teilklasse von Attributen, nämlich den nicht-restriktiven." 
Podlocki, majace piegi,sa zawsze zakompleksione (nichtrestr.) Podlotki majace piegi sa zawse zakompleksione (restr.)

(Weiss $77,5.88$ )

Für die Beispiele für $\mathrm{N}+\mathrm{P}$ bei Weiss 77, S. $222 \mathrm{ff}$ ("generalisierende" Attribute) ist, soweit sie außerhalb der Verwendung im Satz genannt werden, eine Entscheidung hinsichtlich restriktiv : nichtrestriktiv nicht möglich.

6.4.3. $N+P_{1 / k}+V$

Die Betrachtung des Aksl. und des Materials in MS hinsichtlich attributiver Partizipien, die dem Bezugsnomen nachgestellt sind, hat $z$ u berücksichtigen, daß hier grundsätzlich die wahlmöglichkeit besteht zwischen im Aksl. merkmalhaft nichtprädikativer Langform $\left(P_{1}\right)$ und merkmalloser potentiell ambiger Kurzform $\left(P_{k}\right)$.

Die Isolierung, im Russischen ein hochgradig relevantes Strukturmerkmal und anhand der zeichensetzung identifizierbar (mit Einschränkungen möglicherweise bei restriktiven $N+I+P$, die z.T. als nur graphisch isoliert bewertet werden), ist im Aksl. vor Umwertung der Korrelation noch nicht $z u$ gleicher Relevanz ausgebaut und graphisch in den Texten, auch in MS, nicht konsequent bezeichnet.

Grundsätzlich ist auch für die nachgestellte Langform im Aksl. Tendenz zur Isolierung anzunehmen. 86

Das attributive Syntagma $\mathrm{N}+\mathrm{P}_{\mathrm{k} / 1}$ soll durch Beispiele veranschaulicht werden in den Distributionstypen $\mathrm{N}+\mathrm{P}_{\mathrm{k} / \mathrm{l}}+\mathrm{V}$ und $\mathrm{V}+\mathrm{N}+\mathrm{P}_{\mathrm{k} / \mathrm{l}}$.

$86 \mathrm{Vgl}$. Rủžička, SS, S. 339: "Wenn das attributive Partizip vor dem substantiv, mit dem es kongruent ist, steht, ist die grammatische Beziehung beider eindeutig. Das postpositive Partizip hingegen drängt zur Isolierung von seinem Substantiv, im sinne der "obosoblenie", ohne sie immer zu vollziehen. Somit ist die Postposition die merkmallose, Präposition die merkmalhaltige stellung.": vgl.a.a.O. S. 29ff, $272 \mathrm{ff}, 364$ 
6.4.3.1. $N+P_{1}+V$

In dem von Rủzička, SS, S. $327 \mathrm{ff}$ angeführten Material ergibt sich zunächst, daB $N+P_{1}$ mit Substantiv, das bereits individualisiert ist, so daB das Partizip nicht determinierend sein kann, 87 fast ausschlieblich in Zwischenstellung, d.h. $N+P_{1}+V_{1}$ auftritt bei den von uns untersuchten nominativischen Partizipien. Dieser Typ ist insbesondere prädisponiert für die Isolierung, wie sie im Russischen dann konsequent durchgeführt ist; allenfalls in den beiden folgenden Beispielen, für die allerdings die Möglichkeit der Permutation $2 \mathrm{u} N+\mathrm{P}_{1}+\mathrm{V}$ grundsätzlich plausibel erscheint, liegt bei Endstellung von $P$ individualisiertes $\mathrm{N}$ vor:

$\overline{B a}$ nikbtoze ne vidé. nikolize. inoçedy sñ.. spi v lone oči.

to is povédé.

J 1,18 (SS,S.339)

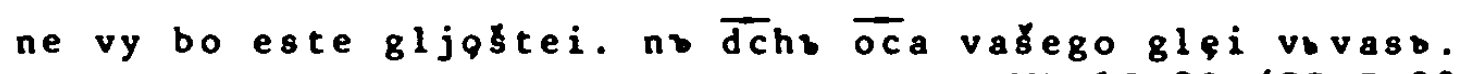
Mt 10,20 (SS, S.337)

Vgl. nichtrestriktives $P$ in Zwischenstellung:

i $\overline{o t c b}$ troi videi vb taine vozdastb tebe ave. Mt 6,4 (SS,S.337)

egda ze $\overline{8 n}$ truoi so izêdy troe imênie su l jubodéicami pride L 15,30 (SS,S.337)

i ty kaf'eronaums. voznesy se do nebsb. do ada sinideri Mt 11,23 (SS,S.338)

otrvěstavı te ijuda prêdavoi ego rete Mt 26, 25 (SS,S.338)

Restriktivität von $P_{1}$ in $Z$ wischenstellung ist nicht auszuschließen, allerdings, verglichen mit $\mathrm{V}+\mathrm{N}+\mathrm{P}_{1}$, weniger ausgeprägt. Nicht vorhanden scheint Restriktivität insbesondere in den Fällen, bei denen die denkbare oder im Material zu belegende Ersetzung durch $P_{k}\left(N+P_{k}+V\right) z u$ einem grundsätzlichen Umbau der struktur führt, nämlich zur untergeordneten Prädikativität des Partizips und Bezug auf das satzschließende Prädikat, d.h. zu Nichtattributivität, wo also die syntaktische Relevanz ${ }^{88}$ des Gegensatzes $P_{l}: P_{k}$ evident wird.

$87 \mathrm{Vgl}$. Rưzička, SS, S. $337 \mathrm{ff}$

$88 \mathrm{Vgl}$.a.a.O. S. $327 \mathrm{ff}$ 
Vgl. z.B.:

i $\overline{c c i}$ chodętei vruchu ne videtb (verborgene Gräber)

L 11,44 (SS, S. 332)

"die Kurzform wäre prädikativ" (Rúzicka, SS, S. 332)

ljudi sêdęstii vo tomé vidéşe svétb velii Mt 4,16 (SS,S.332)

i mqzi drožģtei isa. rqgaachq se emu bijqšte

Vgl. aus MS: 89

L 22,63 (SS,S.332)

blagi ze bog, is-prnva vsa vědyi i ispytajei srodoca, oukrépivy prodannago prékrashnago Iōsifa i stvorivy jego caramogimn jezykomo... javi ó mne velikoje milosrodyje svoje...

(MS, S.90)

Christosn istinny bogo is-prova carostuuei vo véky $i$ na vêky ... obıjavi emou zitie ... otwca ... symeona...

(MS, S. 49)

Der weitgehend formelhafte Charakter dieser Wendungen ist deutlich.

Restriktiv allenfalls:

i ótb inoude starnco chotei prébyvati vosem. piragou da se ne stavi i

(MS, S.75)

\subsubsection{2. $\mathrm{N}+\mathrm{P}_{\mathrm{K}}+\mathrm{V}$}

Die Möglichkeit der Nichtprädikativität ${ }^{90}$ entspricht grundsätzlich der Merkmallosigkeit der Kurzform in der für das Aksl. definierten Korrelation. Allerdings tendiert die Kurzform, wo immer möglich, zu peripherprädikativer Funktion, 91 die sich nicht mehr im Rahmen des attributiven syntagmas beschreiben läßt: gerade in $\mathrm{Zwischenstellung,} \mathrm{bei} \mathrm{nichtrestriktiver} \mathrm{Bezie-}$ hung $z u N$, wird die distinktive Funktion des Gegensatzes $P_{1}: P_{k}$

$89 \mathrm{Vgl}$. M.. S. $62 \mathrm{ff}$

$90 \mathrm{Vgl}$. Rúzicka, SS, S. 69, 43: "Abschließend sei zu diesem Konstruktionstyp bemerkt, daß er die Trennung prädikativer und attributiver stellung erschweren kann"; vgl. a.a.o. S.232ff; Vecerka 61, S. 32ff, 41

$91 \mathrm{Vgl}$. Rủzička, SS, S. $29 \mathrm{ff} \mathrm{zu}$ untergeordnet prädikativen $\mathrm{P}$

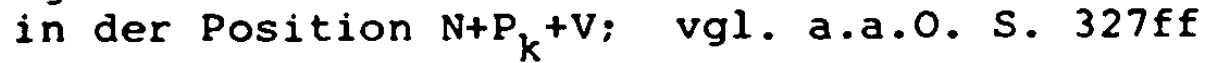


deutlich. Gesichert ist die attributive Funktion von $\mathbf{P}_{k} \mathbf{z} \cdot \mathbf{B}$. bei Koordination mit $P_{1}$ : mnozi ze otb ijudéi. prišdbrei kb marii. i vidévoše eže sotvori is. vèrovał̧ vo nego... 92 J $11,45 / 46$ (SS,S.31) Vgl. sonst:

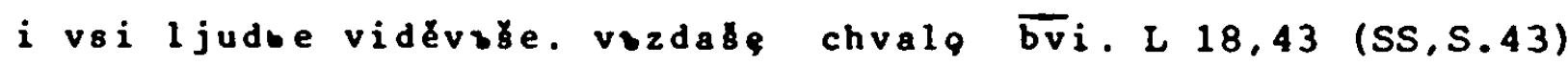
Vgl. demgegenüber prädikativ (Interpunktion!):

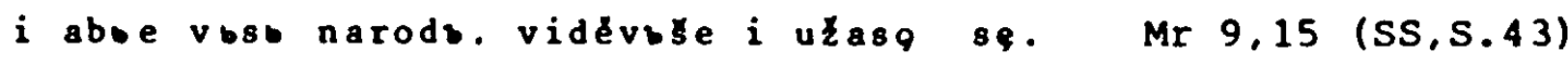
sego radi vosekv konizonikb. naulo se crstviju nbskumu. podobons est cku domovitu Mt 13,52 (SS,5.69) vséko crstuo razdélo se na sq. zapustétr. i voséks gradu. 1 i domb. razdelb senase ne stanetr. Mt 12.25 (SS,S.69)

Bei derartigen Belegen scheint Relativsatzparaphrase grundsätzlich plausibel. Gerade im Hinblick auf die Affinität nichtrestriktiver Erweiterungen von $N$ zu Parenthesesatz oder Koordinierung mit dem finiten Prädikat ist wesentlich, daß bei den vorliegenden Beispielen Koordinierung mit dem satzschließenden Prädikat unmöglich ist bzw. den Sinn der Ausgangskonstruktion entstellt.

Vgl.:

$\rightarrow$ vséko crstro razdelith 89 na sq i zapusteetb (SS.S.69) Rủilka selbst neigt bei den letzten beiden Beispielen der Interpretation als prädikativ mit konditionaler spannung zu, etwa in dem Sinn von

vséko crstvo (aste) razdelits se na se zapusteetn Eine definitive klärung ist hier nicht möglich und auch nicht nötig; entscheidend ist, daB, wenn diese ambigen Beispiele dem Subjektskomplex zugeordnet werden sollen, restriktive Beziehung zu $N$ zugrundegelegt werden muß; eben dies veranschaulicht auch die Unmöglichkeit der Paraphrase mit Koordinierung. Grundsätzlich zeigen diese Belege mit Nachdruck die Ambivalenz der Zwischenstellung des merkmallosen Gliedes der (hier aksl.) Korrelation; vgl, auch (nach Rúzicka eher attributiv): éko bo mluni bliscajqbti se. ots podinskyje. na podinskqjo. suotits se. tako bodets snb csky. vo dno svoi

$$
\text { L } 17,24 \text { (SS.S.91) }
$$

$92 \mathrm{Vgl}$. Ruzicka, SS, S. $275 \mathrm{mit}$ weiteren Beispielen 


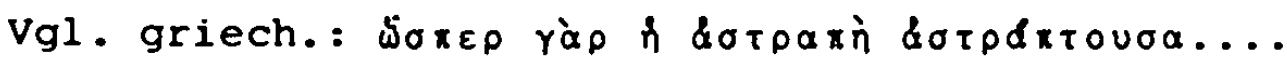

Hier liegt Konfrontierung im Vergleich vor; derartige Belege wären wohl weniger als restriktiv $\mathrm{zu}$ interpretieren denn als formelhafte schmückende Beiwörter (vgl. den griechischen Text) analog $z u$ den aus MS angeführten Belegen. Während in M.,S.61ff so gut wie ausschließlich formelhafte Belege für attributive $P_{k}$ in Zwischenstellung auftreten, ist im Aksl. die Möglichkeit des nichtformelhaften, dann aber restriktiven Gebrauchs von attributiven $P_{k}$ in $Z$ wischenstellung durchaus belegt. Die Bevorzugung der aksl. Langform in Zwischenstellung bei Nichtrestriktivität in dem in Rúzicka, SS, angeführten Material und der nichtprädikativ-attributive Gebrauch der Kurzform insbesondere bei Restriktivität entspricht der grundlegenden Funktion der Langform, eben dann Ausschluß der untergeordneten Prädikativität $z u$ signalisieren, wenn auch eine andere, die untergeordnet prädikative Funktion, grundsätzlich möglich wäre. Umgekehrt besteht für die merkmallose Kurzform insbesondere dann die Möglichkeit zur attributiver Funktion, wenn infolge Restriktivität untergeordnete Prädikativität ausgeschlossen ist. Nach Umwertung der Korrelation bleibt für das jetzt merkmalhafte Gerund nur noch die Möglichkeit der nichtrestriktiven Beziehung gegenüber dem Bezugsnomen. Für das jetzt merkmallose russische Partizip ist bei Nichtrestriktivität und untergeordneter Prädikativität in Zwischenstellung u.E. nicht nur mit einseitigem Bezug auf $\mathrm{N} z \mathrm{u}$ rechnen, sondern auch mit der Möglichkeit der Ambiguität hinsichtlich sekundärprädikativer Funktion mit Bezug gleichzeitig auf das Prädikat. Auch in MS tritt attributives $P_{k}$ in $Z$ wischenstellung auf, sowohl in Kombination von $\mathrm{P}_{1}+\mathrm{P}_{k}$ wie auch alleine. 93 Vgl.:

Ponję̧e oubo clovékoljubco bog neizredenonoju moudrostiju i promys lomb oustrajaje vosa...

(MS, S. 124)

$93 \mathrm{Vgl}$. M. . S. $61 \mathrm{ff}$ 
Vgl .:

poneze oubó clověkoljuboc bog neizrečennoju mudrostiju i promyslomb oustra(ja)jei vsa... (MS,S.129)

...8g8ely oubo prédostoezte ... préstolou.. (MS,S.166)

sego chvalešte diviše se

(MS, S. 71)

Es handelt sich, anders als im Aksl.. nicht eigentlich um restriktive Belege, sondern um ausgeprägt formelhafte wendungen. Eben diese Formelhaftigkeit, der referierend-identifizierende Charakter eines schmückenden Beiwortes, das sich aus der Nennung des übergeordneten $N$ weitgehend vorhersagen läBt (insbesondere bei formelhaften Einleitungen $z u$ den hier ausgewerteten Urkunden) stellt die attributive Interpretation sicher. Diese Formelhaftigkeit des Gebrauchs attributiver $\mathrm{P}_{k}$ in $\mathrm{Zwi-}$ schenstellung ist in MS ungleich deutlicher ausgeprägt als in dem aksl. Material in SS.

6.4.4. $\quad V+N+P_{1 / k}$

Das attributive Syntagma $N+P_{k / 1}$ tritt nicht nur in 2 wischenstellung des Partizips auf, sondern auch in $\mathrm{V}+\mathrm{N}+\mathrm{P}_{\mathrm{k} / \mathrm{I}}$; auch hier sind verbindliche Aussagen über Isolierung nicht möglich, sondern nur Vermutungen $z u$ Analogien mit russischen Typen der Distribution, für die die Isolierung relevant wird.

$6.4 .4 .1 . \quad V+N+P_{1}$

Vgl. aksl. $\mathrm{V}+\mathrm{N}+\mathrm{P}_{1}$ (nichtrestriktiv):

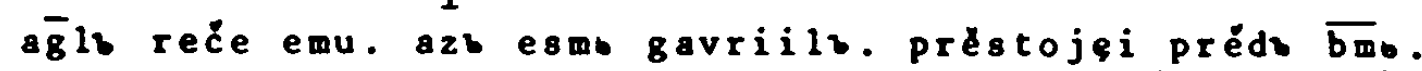
L 1,19 (SS,S.334)

to esto ilie chotei priti Mt 11,14 (SS, 334)

"Das Partizip náhert sich hier der Apposition" (ROz. SS,S.334) i bystb pots ego éko i kaple krove kapljośteje na zemlo L 22, 44 (SS,S.336)

"Der Begriff enthält bereits die in den Partizipien explizierten Merkmale (Ruz.., SS, S. 336) 
Dies gilt bei Kenntnis des zusammenhangs, der den Äußerungen zugrundeliegt, auch für die anderen hier angeführten Beispiele, die damit in die Nähe formelhafter Wendungen rücken.

Restriktivität ist bei Endstellung von $P_{1}$ deutlicher $z u$ unterscheiden als bei Zwischenstellung:

vgl. restriktiv:

voprosisg ze i. knto esth $\overline{\varepsilon k n}$. reky tebé vbzni odrb tvoi $i$

chodi

J 5,12 (SS, S. 329)

blazeno Erevo nosnsee te

L 11.27 (SS,S.330)

aśte vb t'ürè i sidonè. bişe sily byly. byvnşje vh vasis.

drevle ubo...pokajali sebise Mt 11,21 (SS,S.330)

pride vis vitanijo. ideźe bé lazarn umury. egote vrskresi oth

mrotvychb is. J 12.1 (SS,S.331)

glacho éko so estrun isting prorokb gredei vn mirn.

J 6,14 (SS,S.333)

Auch in MS ist die Tendenz zu Restriktivität oder Formelhaftigkeit der nichtprädikativen Langform in Endstellung bei $\mathrm{V}+\mathrm{N}+\mathrm{P}_{1}$ deutlich: (weitere Beispiele M.. S. 62)

vgl. restriktiv:

... nn da imo obladaetostarnco prébyvajei vo semo pirogou

chrama Virzesenija gospodija (MS,S.74,vgl.auch MS,S.8O)

formelhaft:

jako otimetn ōtn njego svoju milostn spass moi christosn vnznesyi se vn slavé kn ōtocou... (MS,S.82)

ako li sie prêstuplju, da me bog snpone i svetabogorodica $i$

vsi sveti ugoždoši bogu ôto věka (MS,S.25)

so auch ougoždivšei in MS,S.166

Vgl. hierzu gegebenenfalls auch:

imamn bo vnsi zivuštei na zemli somrntn vokousiunségrobu pré-

dati telesa nasa (MS, S.100) 94

Zwischenstellung der nichtprädikativen Langform überwiegt in MS bei weitem, in Endstellung treten entweder restriktive oder

94 Vgl. Rúzicka, SS, S. 299: "Verbindung vasi + Vollpartizip: Sichere, für jeden Fall geltende Kriterien zur Feststellung, ob das pronomen die absolute und das Partizip die attributive Funktion einnimmt, gibt es nicht." 
weitgehend formelhafte Belege, die schmückenden Beiwörtern nahekommen, auf.

6.4.4.2. $\quad \mathrm{V}+\mathrm{N}+\mathrm{P}_{\mathrm{k}}$

In MS hatten wir kaum Belege für attributive Funktion bei $\mathrm{V}+\mathrm{N}+\mathrm{P}_{\mathrm{k}}$ gefunden. In dem von Rúzička untersuchten aksl. Material finden sich einige Beispiele, die nicht immer eindeutig sind. 95

Vgl.:

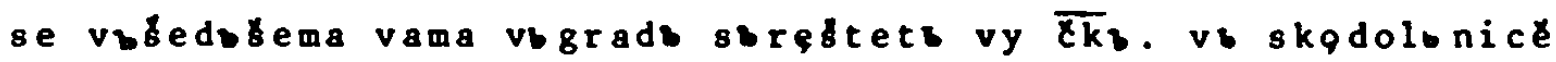
vodo nese L 22.10 (SS,S.163. Beispiel Nr. 672)

"Das einer Determinierung bedurftige $\overline{c k}$ zieht das Partizip in attributive stellung." 96

Demgegenüber vermutlich prädikativ:

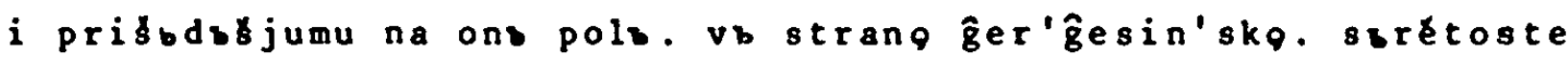
i duva bésuna. ot grebiłto ichs. i schodęta ljuta zêlo. Mt 8,28 (SS.S.162/163,Beispiel Nr. 671)

Bei Konstruktionen mit uberwiegend formelhaftem imy $/$ imgste (SS.S.167ff) besteht Tendenz zur Neutralisierung des Gegensatzes attributiv : prädikativ:

estb ze vo ermẻch na ovoli kapeli. éże naricaetb se evreisky fezda. petb prituorb impsti J 5,2 (SS,S.167)

i pristopiše kb nemu narodi mnozi. imqste sb sobojq chromy... Mt 15.30 (SS.S.167)

ons be suetiluniks gore i suote J 5,35 (SS,S.23.3)-formelhaft

"Ein partizipielles Merkmal eines substantivischen Prädikats-

95 Vgl. Rúzicka, SS, S. 163: "Die Inversion von Prädikat und subjekt berührt das periphere Prädikat indirekt: es ruickt näher zum Subjekt. Wenn dann das Partizip nicht zum Prädikat in Beziehung gesetzt ist, wie in Beispiel 672, dann nähert es sich einer attributiven Determination. Doch ließe sich eine attributive Funktion des Partizips in Beispiel 672 gegenuber einer prädikativen in Beispiel 671 gramatisch nicht zuverlässig begründen."; vgl.a.a.0. S. 354 u. 232

96 Rủzicka, SS, S. 163 
nomens kann bei Identifizierungen keine eigene periphere Prädikation mit dem Subjekt eingehen." (Rủz..SS,S.233) restriktiv:

blazenoe Erevo nostise ts

L 11,27 (SS, S. 233)

(s.o.. vgl.: blaženo ćrevo nośnśee te (SS.S.330))

"Ein partizipielle Merkmal des Subjekts ist Gegenstand und nicht Teil der identifizierenden Prädikation" (Rủż.,SS,S.233) 97 Vgl.:

i issodn is vidé narodw mnogm. i mili emu byše. za ne bèachq éko ovoce ne imoste pastucha Mt 6.34 (SS,S.234)

Auf weitere, problematische Belege soll hier nicht eingegangen werden. 98

In jedem Fall liegt vor $\mathrm{N} \mathrm{P}_{k}$ mit restriktivem oder formelhaftem Gebrauch von $\mathrm{P}_{k}$. In Identifizierungssätzen scheint der funktionale Gegensatz zwischen $P_{l}$ und $P_{k}$ weitgehend aufgehoben. $P_{1}$ sichert die Interpretation als nichtprädikativ insbesondere dann, wenn weder formelhafter Gebrauch noch restriktive Beziehung vorliegt: in diesen Fällen tendiert $P_{k}$ deutlich zur Prädikativität.

Angesichts des oft fragmentarischen Satzbaus und der inkonsequenten Interpunktion ist es nicht ganz leicht, in dem in MS gesammelten Material klare Belege für nichtprädikativen Gebrauch der Kurzform anzufüren. 99 Nicht so sehr sind für uns in diesem Zusammenhang die Fälle von Interesse, bei denen entsprechend den aus dem Aksl. bekannten Bedingungen der Realisienung der Korrelation nichtprädikatives $P_{k}$ auf $P_{l}$ folgt wie z.B. in:

tyi ze istn jesi ... stuorivyi ... outvrndivii ... prijem(yi)

... postavivn... rek...

(MS,S.25/26)

97 Vgl. Rủzicka, SS, S. 233: "Attributives und prädikatives Partizip lassen sich leichter abgrenzen in Identifizierungssätzen: Die Einheit des Subjekts und/oder des nominalen Prädikats, mit und in ihren partizipiellen Modifizierungen, impliziert die Attributivität der Partizipien."

$98 \mathrm{Vgl}$. a.a.O., S. $234 \mathrm{ff}$

$99 \mathrm{Vgl}$. M., S. $61 \mathrm{ff}$ 
Vgl. mit attributivem $P_{k}$ :

vo sem Le chrame spasa nałego zd\& da postavlajutw se vosi

kralije, chotelte byti drulave si ... (MS,S.13)

sego radi toltimb se i myi podvizoubte se otozemlonyichok k nebesnymb naipate syati ...

(MS, S.73)

sego radi tobtiimb se i myi, podovizulte se ötremlunyich na nebesnaja, naipace sly $\mathbf{a t i}$...

(MS, S. 78)

so auch podvyžouste in MS,S.121. 
Auch in neueren Arbeiten zu Partizipial- und Cerundialstrukturen besteht die deutliche Neigung, sich bei der Klassifizierung im wesentlichen $z u$ beschränken auf die Zuordnung zum Subjektsoder Prädikatskomplex, die fraglichen Strukturen z.B. als attributiv bzw. adverbial zu klassifizieren und sich dabei auf die Analogie $z u$ vorausgesetzten und als einheitlich unterstellten syntaktischen Kategorien $z u$ berufen.

Deren formale und semantische "Varianten" werden dann in den Bereich der Performanz und/oder einer nicht näher präzisierten Semantik verwiesen und entziehen sich einer strukturierung. Dies ergibt sich zunächst einmal aus dem zugrundegelegten Modell; wenn nur die binäre Gliederung in subjekts- und Prädikatskomplex vorgesehen ist, wird eine weitergehende systematische Differenzierung innerhalb dieser Komplexe und insbesondere von Strukturen, die sich eben nicht hinreichend beschreiben lassen im Hinblick auf ein ubergeordnetes hauptrangiges Satzglied, schwierig.

Natürlich wird auch in Arbeiten, die sich beschränken auf die Klassifizierung nach der Zugehörigkeit zu NP : VP, im Einzelfall verwiesen auf formale und semantische Besonderheiten, die über diese Klassifizierung hinausgehen, nur muß dabei offen bleiben, was hierbei das einzelne Beispiel kennzeichnet und wo strukturbildende Relevanz vorliegt.

7.1. Zum relativen Tempus

Eine wesentliche Funktion untergeordnet prädikativer Partizipial- und Gerundialstrukturen ist der Ausdruck relativer Zeitstufen. 1 Deshalb liegt es zunächst nahe, für die Differenzierung des Verhältnisses von Partizip/Gerund zum übergeordneten Prädikat die Zeitenfolge und in Zusammenhang hiermit Aspekt- und

1 Vgl. z.B. Panzer 75, S. 119; Barnet 65; Isacenko 75, S. $324 \mathrm{ff}, 332 \mathrm{ff}$ 
Tempusmerkmale heranzuziehen. Diese sind immerhin ihrer Form nach identifizierbar und bieten eine äuBeren Anhalt zur Gliederung des aufgeführten Materials. Für das Russische wird die Grundregel etwa so angegeben, daß für den Ausdruck der Gleichzeitigkeit Partizip und Gerund des Präsens zur Verfügung stehen und für den Ausdruck der Vorzeitigkeit pf. Präsensgerundien und Präteritalgerundien.

Noch wichtiger als der Hinweis darauf, daB hier, jedenfalls z.B. für das Russische ${ }^{2}$ und Polnische, noch weitere Untersuchungen nötig sind, ist die Frage, ob die Zeitenfolge mit ihren vielfältigen Bedingungen, insbesondere Tempus und Aspekt. aber auch Wortstellung und z.B. lexikalischer Semantik der beteiligten Komponenten, überhaupt geeignet ist, strukturtypen systematisch zu klassifizieren.

Die potentielle vieldeutigkeit von russischen pf. Gerundien ist bekannt. 4 Nicht für jede Partizipial- und Gerundialkonstruktion kann uberhaupt gleichermaßen Unterordnung unter die übergeordnete Prädikation mit klar definierter zeitenfolge vorausgesetzt werden: es ist mit der Möglichkeit $z$ u rechnen, daß neben dem Zeitbezug auf die Prädikatshandlung der Bezug zum zeitpunkt der Aussage relevant wird. 5

Zumindest vorläufig ist es eher möglich, typische fälle des

2 Vgl. Mulisch 75, S. 198; Ružicka 66, S. 47; zum Russ. allg. vgl. Panzer 75, S. 139

$3 \mathrm{Vgl}$. Weiss 77, s. $307 \mathrm{zu}$ pf. und ipf. Gerundien im Poln.: "Die strikte Scheidung der beiden Formen nach ihrem relativen Zeitbezug entpuppt sich jedoch als Dogma, dem die Sprachwirklichkeit längst nicht immer entspricht.": a.a.0. S. 313: "Im ubrigen ist nochmals darauf hinzuweisen, daß bezüglich des zeitreferentiellen Gebrauchs von Part-II-Konstruktionen kein allgemeiner Konsens der Sprachbenützer besteht, was wiederum mit ihrer peripheren Rolle in der Sprachverwendung bzw. ihrer mangelnden Verankerung in der Sprachkompetenz zusammenhängen dürfe."

$4 \mathrm{Vgl}$. 2.B. Brave 40; Isacenko 75, S. 326: Kornilov 67, S. $91 \mathrm{ff}$

5 Vgl. Kornilov 67; ders. 64; Brave 40, S. 25ff; Paffen II, S. 365: "Für logisch unabhängige, selbständige Gerundialsätze dagegen, die den Wert eines Hauptsatzes haben, gilt auch selbständige Aspekt- und Tempuswahl. Dadurch werden die Verhältnisse beträchtlich verwickelter."; vgl. hierzu a.a.o. S.358ff, 369ff: Paffen III,S.208; Gvozdev I 73, S. $386 \mathrm{ff}$ 
Aspekt- und Tempusgebrauches anzufüren in Interdependenz mit anderen Kriterien, als eine zusammenfassende Erklärung der semantischen struktur des Prädikatskomplexes unter dem übergeordneten Gesichtspunkt der Zeitenfolge zu gewinnen. Dies wird anschaulich z.B. in dem umfangreichen Material bei Mulisch 75, S. 196ff; bei dieser Aufzählung möglicher "AspektTempus-Bedeutungen" wird eine Zuordnung semantischer strukturtypen allgemeinerer Art nicht ersichtlich, sondern eher umgekehrt die Relevanz von Wortstellung und Kontext für die Ausformung des relativen Tempus.

Dementsprechend unterscheiden z.B. Vondrák, Vecerka und Rudnev zwischen Zeitenfolge und Funktion innerhalb des Prädikatskomplexes, und dementsprechend ordnet Rüzicka für das Aksl. die Beschreibung nach Tempus und Aspekt der Klassifizierung nach Wortstellungstypen und deren semantischer signifikanz unter. ${ }^{6}$

Für unsere Zwecke sollen demnach die Aspekt- und Tempusproblematik zurückgestellt und Fragen des relativen Tempus als allgemeineren semantischen strukturtypen untergeordnet angenommen werden. Bei der Untersuchung des dieser Arbeit zugrundeliegenden sprachlichen Materials ergab sich keine Aussicht auf eine durchgängige semantische Klassifizierung auf der Gurndlage von Tempus und Aspekt. Den umgekehrten Weg gehen für das slk. J. Rüzicka und Kačala, insofern hier nur bei Gleichzeitigkeit doplnok-Funktion angenommen wird, bei Vorzeitigkeit Funktion als adverbiale Bestimmung. Jedoch läßt sich die behauptete semantische Unterscheidung dieser beiden Funktionstypen bei Kacala nicht auf den Unterschied der Zeitenfolge reduzieren. Miko und Křizková ordnen demgemäß Fragen der Zeitenfolge einer allgemeineren syntaktisch-semantischen Klassifizierung unter. Grundsätzlich ist daran zu erinnern, daß die sogenannten prädikativen Kategorien zunächst einmal nur der fakultative morphologische Ausdruck der syntaktischen Funktion der Prädikation sind. Dies hat auch die Umschreibung der untergeordnet

6 Ktizková 69, Synt.ch., S. $121 \mathrm{ff;} \mathrm{Rưzicka,} \mathrm{SS,} \mathrm{S.} 13 f \mathrm{f}$ 
prädikativen Funktion von Partizipial- und Gerundialstrukturen anhand der angenommenen Unterordnung unter die prädikativen Kategorien der satzschließenden Prädikation zu berücksichtigen. Auch wenn wir uns beschränken auf Fälle mit Subjektsidentität, ist $z$ u berücksichtigen; daß nicht immer Unterordnung des untergeordnet prädikativen Gerund unter die Kategorie der Person des satzschließenden Prädikats gilt lvgl. Wendungen in der Funktion von Satzadverbien wie korotko govorja, mjagko govorja usw.). Nicht immer gilt der Modus des satzschließenden Prädikats auch für das untergeordnet prädikative Partizip/Gerund, ${ }^{7}$ und nicht in jedem Fall gilt temporale Unterordnung unter das satzschließende Prädikat.

7.2. Adverbiale Funktion in der binären syntaktischen Gliederung

Die ungelöste Problematik einer strukturellen Differenzierung des Prädikatskomplexes wird deutlich z.B. bei Jacobsson $69 .^{8}$ Er nimmt die Unterschiede hinsichtlich der zeitenfolge und anderer semantischer Relationen wie Enthaltensein/Parallelität der Nebenhandlung sowie formale Kriterien, die distributionell mit der Stellung des Gerund vor oder nach dem Prädikat verknupft sind, nicht zum Anlaß, den Begriff der "adverbialen" Funktion selbst zu differenzieren, sondern vermutet hier allenfalls stilistische Zusammenhänge.

Ein ahnlich undifferenzierter Begriff der adverbialen Funktion von Partizipial- und Gerundialstrukturen, erweitert um den zusätzlichen Hinweis auf die untergeordnete Prädikativität, liegt z.B. auch noch zugrunde bei Babby 75, Mulisch 75 u.v.a. Gabka 76 z.B. unterscheidet neben Adverbialbestimungen, die obligat sind infolge Valenzeigenschaften des ubergeordneten Verbs, freie Adverbialbestimmungen mit Bezug auf das Satzgan-

7 Zum Poln. vgl. Weiss 77, S. $300 f f$

$8 \mathrm{Vgl}$. Jacobsson, Gö. 69, S. $52 \mathrm{ff}$ und die dort angeführte Literatur 
ze oder aber auf das Prädikat. 9 Für isolierte Partizipien und Gerundien wird deren "adverbiale" Bedeutung nur nach varianten wie modal, konditional usw. an Beispielen veranschaulicht, nicht aber nach der für Adverbialstrukturen angedeuteten Differenzierung nach Typen.

Fuir das Deutsche unterscheiden Rath 71, Bungarten 76 und Filipović 77 zwischen der Zuordnung zum Subjekts-oder zum Prädikatskomplex, zu explizieren durch Relativsatz bzw. adverbialen Nebensatz. Die Subklassifizierungen der adverbialen Funktion erfolgen im wesentlichen nach den bei der Paraphrasierung auftretenden Konjunktionen, ihr Vergleich macht die Subjektivität einer solchen Klassifizierung anschaulich.

Unklar bleibt hier zunächst einmal die Frage des Satzbezuges. Rath geht von Bezug nur auf das Prädikat aus, Bungarten sieht für attributive und adverbiale Partizipialkonstruktion zusätzlich die Möglichkeit des Satzbezuges vor, ohne sie zu explizieren, Filipović nimmt für alle adverbialen Partizipialkonstruktionen Satzbezug an. 10

7.2.1. Paraphrase durch adverbialen Nebensatz

Ein Blick auf die umfangreiche Literatur zu Adverbialbestimmungen zeigt, daß diese weiterer Differenzierungen bedürfen und da $\beta$ ihr Verhältnis $z u$ den sogenannten adverbialen Nebensätzen noch zu klären ist (s.0.). 11

Wenn bei der Interpretation der Partizipial- und Gerundialstrukturen zurückgegriffen wird auf die Satzgliedposition "adverbiale Bestimmung", müssen auch die damit implizierten strukturellen Unterscheidungen angewendet werden. Die Paraphrasierung der Partizipial- und Gerundialstrukturen

$9 \mathrm{Vgl}$. Gabka 76, S. 203ff, $214 \mathrm{ff}$; AG 70, S. 624ff, $644 \mathrm{ff}$ $10 \mathrm{Vgl}$. Bungarten 76, S. 150ff, 173ff; Filipovic 77, S. llff, vgl. aber a.a.0. S. $49 \mathrm{ff} z \mathrm{u}$ "Satzattributen", S. $188 \mathrm{ff} z \mathrm{z}$ "modalen" Partizipien

11 Rohrer 71, S. 198 
durch sogenannte "adverbiale Nebensätze" wird angeführt einerseits zum "Nachweis" des adverbialen Satzgliedstatus der zu erklärenden Strukturen, andererseits zur Differenzierung der vorausgesetzten adverbialen Funktion nach semantischen Varianten wie temporal, kausal, modal u.v.a..Beides ist problematisch: Ersetzbarkeit sichert noch nicht Aquivalenz, auch die adverbialen Nebensätze sind keine homogene Klasse. Analog $\mathrm{zu}$ unseren Uberlegungen zur Relativsatzparaphrase folgt aus der Tatsache der Ersetzbarkeit entgegen $z$. B. Babby 75 noch nicht, daß etwa explizite adverbiale Nebensätze den kondensierten Strukturen im Sinne einer Ableitungsgeschichte zugrundelägen, sondern allenfalls, daß beide einen gemeinsamen Ausgangspunkt der Ableitung haben können. Die semantische Unbestimmtheit der Kondensate hinsichtlich temporaler, kausaler, konditionaler usw. Varianten läßt sich nicht hinreichend als oberflächenhaftes Resultat der Tilgung tiefenstrukturell vorhandener Information beschreiben, vielmehr muß zumindest die Möglichkeit angenommen werden, daß diese Unbestimmtheit (die graduell variabel ist je nach den Bedingungen des jeweiligen Kommunikationsaktes) vom Sprecher intendiert ist. AuBerdem kommt als Paraphrase nicht nur konjunktionale Hypotaxe infrage. 12 Die oberflächensyntaktische Unterscheidung von Parataxe und Hypotaxe kann für die Tiefenstruktur richt gleichermaßen vorausgesetzt werden. 13

Der Versuch, anhand der Paraphrasierung durch adverbiale Nebensätze zu einer Differenzierung des Prädikatskomplexes bzw. einer systematischen Klassifizierung der Partizipial- und Gerundialstrukturen $z u$ gelangen, ist aussichtslos. ${ }^{14}$ Die auf diesem

12 Vgl. z.B. Sint.r.j. 74, S. $137 \mathrm{zu}$ Parataxe oder Hypotaxe als möglichen Paraphrasen; vgl. Adamec II 75, S. 177; Rudnev 63, S. 180; Vondrák 28, S. 401ff

$13 \mathrm{Vgl}$. Dressler in Daneš 74 (ed.). S. 98ff: Petöfi 71.5.195ff Hartung 73

$14 \mathrm{Vgl}$. Weiss 77, S. 315ff, 327; Mulisch 75, S. 206ff differenziert quantitativ: am häufigsten tritt demnach temp. und mod. Beziehung auf, seltener kaus. und kond., am seltensten fin. und konsek. Beziehung 
Weg erschlossenen semantischen Varianten bleiben beliebig. Dies zeigt sich auch im unscharfen Charakter dieser semantischen Varianten, insbesondere erweist sich die Kategorie "modal" als dehnbar. 15 Die Klassifizierung aufgrund von Paraphrasen läuft Gefahr, die Klassifizierung einer Beziehung zu verwechseln mit der Klassifizierung eines Lexems, der Konjunktion.

Wie die neuere Adverbialsyntax und -semantik zeigt, liegen den expliziten Varianten der Adverbialstrukturen wie temporal, kausal usw. Typen ${ }^{16}$ und letztlich Prädikationen unterschiedlicher Reichweite zugrunde. Bei den Aufzählungen semantischer Varianten von Partizipial- und Gerundialstrukturen in "adverbialer Funktion" verstellt der unmittelbare Rückgriff auf die expliziten Paraphrasen als mögliche Varianten der Realisierung den Blick auf die zugrundeliegenden Typen der Konnexion. Die Frage ist, ob man sich aufgrund der Aussichtslosigkeit einer Klassifizierung nach semantischen Varianten uberhaupt einer semantischen Differenzierung des Prädikatskomplexes $z u$ begeben hat, wie dies Weiss nahelegt.

Zur Resignation hinsichtlich der Möglichkeiten einer Differenzierung trägt weniger die nicht $z u$ bestreitende Ambiguität bei als die Tatsache, daß hinsichtlich der semantischen Kategorien, für die Ambiguität gelten soll, keine Klarheit besteht. Unklar bleibt notwendigerweise die Konstituierung dieser semantischen Varianten bei fortbestehender Unklarheit hinsichtlich des Begriffs der sprachlichen Bedeutung und der Aquivalenz. Eine zuordnung von oberflächenstrukturen im sinne der asymmetrischen Zeichenrelation ist so nicht möglich.

$15 \mathrm{Vgl}$. als Beispiel für die Beliebigkeit der "modalen" Interpretation Fedorov 72, S. $92 \mathrm{ff}$, der als "obstojatel'stva obraza dejstvija" zusammenfaßt: kacestvo dejstvija, sobstvenn. obraz dejstv.. sravnitel'nyj ottenok, sposob dejstv.. sovmestnost'/razdel'nost', rezul'tat, sostojanie

$16 \mathrm{Vgl}$. auch die Ansätze zur Gruppierung semantischer Varianten 2.B. bei Kŕizková 73, Weiss 77, S. 342, 346, Grochowski 75: zur Unterscheidung allgemein-logischer und spezifischer semantischer Relationen vgl. Bungarten 76 , S. $175 \mathrm{ff}$ 
7.3. Klassifizierung als "prädikativer Determinant"

Partizipial- und Gerundialstrukturen im Rahmen des Prädikatskomplexes werden spezifiziert aufgrund des Bezugs auf subjekt und Prädikat gleichzeitig. So weitgehend der "Doppelbezug" untergeordnet prädikativer Partizipien und Gerundien anerkannt ist, so problematisch bleibt seine Definition, wie schon die terminologische vielfalt zeigt.

Als Termini treten u.a. auf predikatinny opredelitel', predikativnoe obstojatel'stvo, doplněk, tavtosub-ektnoe dobavlenie, priskazuemoe, vtorostepennoe skazuemoe (sekundäres Prädikat) u.a.m. Diese noch durchaus unvollständige Aufzählung enthält morphologische, syntaktische und semantische Implikationen, die beinahe von Fall zu Fall verschieden gewertet werden. Dabei besteht weitgehend Ubereinstimmung hinsichtlich der untergeordnet prädikativen Beziehung zum Subjekt, unterschieden wird insbesondere Koordinationsbeziehung und Determinationsbeziehung 17 gegenüber dem Prädikat.

Transformationell wird der "Doppelbezug" üblicherweise so dargestellt, daß die kondensierte struktur abzuleiten ist von einem Konstituentensatz, dessen subjekt referenzidentisch ist mit dem des Matrixsatzes und auf dem Weg zur oberfläche getilgt wird. 18 Dem kann auch die Paraphrasierung durch zwei koordinierte Sätze mit identischem und gegebenenfalls zu tilgendem subjekt entsprechen. Es ist aber daran zu erinnern, daB hiermit zunächst einmal wirklich nur über die außersprachliche Tatsache der Referenzidentität des subjektes etwas besagt ist und noch nichts über die spezifische Funktion der eingebetteten Struktur bzw. über die Position der Einbettung. Die von Kacala als entscheidendes Kriterium angefuhrte Koordinationstransformation ist bei gleichem außersprachlich Bezeichneten und sehr unterschiedlichen Funktionstypen im Satz möglich, z.B. für

$17 \mathrm{Vgl}$. z.B. Kacala 71, S. 10-53: Svetlik 70, S. 225ff; sint. r.j. 74, S. $133 \mathrm{ff;}$ Adamec II 75, S. 7ff, $21 \mathrm{ff}$

$18 \mathrm{Vgl}$. z.B. Rủzicka 66, S. 45; Weiss 77, S. 338ff; Adamec II 75. S. $173 f f$ 
Attribut zum Subjekt (bzw. Apposition) und sekundäres Prädikat. Sie reflektiert nicht die funktionale spezifik der infragestehenden Struktur.

"Doplnok"-Funktion sieht Kacala"19, J.Rüżička folgend, nur bei Gleichzeitigkeit von Partizipial- und Gerundialstrukturen. Hier wird aus Gleichzeitigkeit auf Gleichordnung geschlossen im Unterschied zur determinativen Beziehung, in der das Gerund als adverbiale Bestimung klassifiziert wird. Andererseits schließt Kaçala auch für doplnok zusätzliche semantische Nuancen wie kond., kaus.. konzess. nicht aus. U.E. ist die Unterscheidung von (unechter) Koordination und Determination bei Kacala willkürlich: vgl. die folgenden Beispiele nach Kacala 71, S. 187 :

Stál tak chvilu s roztiahnutymi nohami ("adv. Bestimmung") Sedela doma s rozbolenou hlavou ("doplnok")

"Subjektsidentität" reicht nicht aus zur Unterscheidung der $\mathrm{Zu}-$ ordnung zum Subjekts- oder Prädikatskomplex sowie zur Differenzierung des Prädikatskomplexes selbst insbesondere hinsichtlich der Unterscheidung des Bezugs untergeordnet prädikativer Strukturen nur auf das Prädikat oder auf das Satzganze, wie sie z.B. auch von Bartsch (s.u.) in Zusammenhang mit sogenannten "adverbialen Attributen", die sich z.T. wie "relationale", z.T. aber wie "modale" Strukturen verhalten, diskutiert wird.

Solange die hier zugrundeliegenden funktionalen Unterschiede nicht präzisiert sind, ist es nicht ergiebig, "doplnok" und "adverbiale Bestimmung" gegenüberzustellen als mögliche Funktionstypen für Partizipial- und Gerundialstrukturen. Subjektsidentität gilt in beiden Fällen.

Grundsätzlich zeichnen sich in der Literatur zu doplněk, prädikativem Determinant usw. ähnliche Ansätze zur Differenzierung des Prädikatskomplexes wie bei Adverbialstrukturen ab, und analog besteht die Schwierigkeit einer konsequenten Anwendung auf unsere Partizipial- und Gerundialstrukturen, solange die funktionstypkonstituierenden Bedingungen nicht geklärt sind.

$19 \mathrm{Vgl}$. Kačala 71, S. 20ff, 147ff, 153ff, 159 
So unterscheidet z.B. Uličný zwischen obligatem "OPD" bei nicht voll bedeutungstragenden Verben (nicht teilbar) und fakultativem " $D$ " (zweibasig) bei vollbedeutungstragenden Verben, mit der Möglichkeit direkter und indirekter Determination durch D.

Ahnlich setzt Mrázek die problematische Unterscheidung von Kopula, syn- und autosemantischen verben voraus. ${ }^{21}$ Bei autosemantischem Verb kann der "prädikative Determinant" in ergänzender Funktion stehen (Merkmal abhängig von der Prädikatshandlung). Fundiert scheint dieser Gegensatz hier referentiell zu sein. Mrázek spricht u.a. vom Zusammenfall von "Substanzen"?2 Die Relativität solcher Unterscheidungen wird sichtbar bei den Belegen z.B. Svetliks 23 für Gerundien zur Angabe einer parallelen Handlung gegenüber solchen zur Angabe eines Merkmals der Prädikatshandlung, sowie der Zuordnung von Varianten $z u$ diesen Typen, wenn z.B. die Angabe der Bedingung und der Art und Weise der Haupthandlung gleichermaßen als "Merkmal" der Prädikatshandlung genannt werden.

Adamec 24 füht unter dem Oberbegriff (fak.) "tavtosub-ektnoe dobavlenie" nichtattributive Partizipien und Gerundien an, verweist auf die mögliche Affinität zu "Situativ" (mit Satzbezug) und auf die semantische Unterscheidung hinsichtlich Angabe einer parallelen Handlung: zusatzliche adverbiale Beceutungsnuance. Mit wechselnder Terminologie wird unterschieden zwischen fakultativen kondensierten Strukturen, die ein Merkmal der übergeordneten Handlung angeben und mit dieser zusammen als Einheit einer Handlung $z u$ werten sind und anderen, bei denen zwei voneinander verschiedene Handlungen in Beziehung zueinander gesetzt sind. Nur zeigen die Umschreibungen dieses semantischen Gegensatzes und die Beispiele, daß er durchaus nicht

$21 \mathrm{Vgl}$. Mrázek 58, S. 16ff, $21 \mathrm{ff}$; ders. 61, S. 56

22 ders. 58, S. 23

$23 \mathrm{Vgl}$. Svetlik 70, S. $267 \mathrm{ff}$

$24 \mathrm{Vgl}$. Adamec II 75, S. $75 \mathrm{ff}, 171 \mathrm{ff}, 185 \mathrm{ff}$ 
immer gleich verstanden wird und daß er kaum zu explizieren ist.

Die Frage nach der Begründbarkeit der behaupteten zugrundeliegenden Strukturtypen und damit auch der Anwendung auf die Partizipial- und Gerundialstrukturen, die sich vorläufig negativ nur durch Hinweise auf ihren syntaktisch nichtobligaten und ambigen Charakter umschreiben lassen, bleibt.

\subsection{Signifikanz der Wortstellung}

Aussichten auf eine systematische Differenzierung statt einer bloßen Aufzählung semantischer Varianten oder Mutmaßungen hinsichtlich semantischer Typen ergeben sich auf der Grundlage der Klassifizierung nach formalen Kriterien und der Frage nach ihrer Signifikanz. Typen der Wortstellung signalisieren notwendigerweise nicht die üblichen Varianten, sondern allenfalls Typen der Konnexion.

Z.B. interpretiert Miko 72 für das slk. alle Transgressive in Analogie zu Adverbialstrukturen. ${ }^{25}$ Diese werden gemäß ihrer lexikalisch expliziten Bedeutung in der Konstituentenstruktur in unterschiedlicher Nähe zum Zentrum der Prädikation hierarchisch angeordnet. Für Transgressive, wo die Frage nach temporaler. modaler, kausaler usw. Bedeutung ja nicht ein Lexem, sondern eine Beziehung betrifft, wird aus der Wortstellung auf mögliche Varianten geschlossen. Temporale und kausale Interpretation wird dabei für Voranstellung gegenüber dem Prädikat vorgesehen, Nachstellung signalisiert modale Bedeutung.

Vgl . bei Verwendung derselben Lexeme:

idúc domov, spievali si do kroku (temp.)

Iśiel, spievajúc si do kroku, domov (mod.) (Miko, S.119)

Die Wortstellung wird damit relevant für die Position in der Konstituentenstruktur. Allerdings: Wenn Miko auch hinweist auf unterschiedliche Typen ("Unabhängigkeit : Identität" der Handlungen), von den "limitierenden" Adverbien abgesehen, die in

$25 \mathrm{Vgl}$. Miko 72, S. $25 \mathrm{ff}, 34,117 \mathrm{ff}$ 
unserem Zusammenhang keine Rolle spielen sollen, erfolgt für sämtliche Varianten Einordnung unter VP, d.h. es ist hier nur Bezug auf das Prädikat vorgesehen, nicht auf das Satzganze. Musić $35^{20}$ erörtert die Verteilung semantischer Varianten auf Wortstellungstypen von skr. Gerundien. Auch hier soll bei Nachstellung modale (Explikation durch tako da), bei Voranstellung gegenüber dem Prädikat insbesondere temporale oder kausale, seltener z.B. konditionale, konzessive u.a. Beziehung vorliegen. Mit einer irritierenden Terminologie wird aufgrund der Wortstellung auch eine Verallgemeinerung $z u$ Typen der Konnexion umschrieben, die sich U.E. so zusammenfassen läßt, daß bei Voranstellung des Gerund meist die Prädikatshandlung vor dem Hintergrund der Gerundialhandlung gesehen wird, bei Nachstellung ungekehrt die Gerundialhandlung vor dem Hintergrund der Prädikatshandlung.

Belić polemisiert in diesem Zusammenhang mit unnötiger Schärfe gegen Musić. Fehlende Kongruenz ist kein Argument gegen den von Musić angenommenen Doppelbezug: im Ergebnis komnt Belić zu einer ähnlichen Klassifizierung wie Musić. Belić unterscheidet 27

temporale Bedeutung bei otac placuri zapita zmiju und modale Interpretation für siromach otide placuci.

Bei Miko, Musić u.a. wird also versucht, von Wortstellungstypen auf die üblicherweise beliebig aufgezählten Varianten $z u$ schließen. Dabei zeichnet sich die Verallgemeinerung $z u$ Typen der Konnexion ab. Die naheliegende Konsequenz daraus ist, eben nicht mehr im ersten Schritt nach Varianten zu klassifizieren. sondern nach Typen.

Rath 71 z.B. unterscheidet im Deutschen innerhalb der "modalen" Variante (Paraphrase durch indem) die Typen Zerlegung eines Vorgangs : Verbindung zweier verschiedener Vorgänge. 28

$26 \mathrm{Vgl}$. Musić 35, S. 128ff, 135ff, 143ff, 154ff; ähnlich PeSikan 59, S. $91 \mathrm{ff}$

27 Belic 33/34, S. 196ff; ders. 69, S. $191 \mathrm{ff}$

28 Rath 71, S. 153ff: vgl. hierzu auch Bartsch 72, S. $173 f f$ 
Rath selbst begründet dies eher referentiell; zunächst unterscheiden sich aber die angeführten Belege durch die Wortstellung (a.a.O. S. 155):

ein Vorgang: Wie jene Soldaten, welche die Tempel Moscheen und die Lamas kleine Kamele nanten, entstellen sie die wirklichkeit, indem sie lateinische Formeln auf sie anwenden.

zwei Vorgänge: Indem sich immer nur die vitalsten und den Umweltbedingungen am besten angepaßten Formen fortpflanzen und erhalten, kommt es zu einer gleichgerichteten Summation veränderter Eigenschaften.

ein Vorgang: Achilles gleich sollte Julian eines frühen Todes auf dem Schlachtfeld sterben, vom speer eines Feiglings durchbohrt.

Vgl.: a) vom Speer eines Feiglings durchbohrt, starb Julian.

b) Julian starb, vom Speer eines Feiglings durchbohrt.

Die außersprachliche Situation ist dieselbe. Die unterschiedliche Wortstellung signalisiert den durch den Hinweis auf die Ambiguität der Konjunktion indem umschriebenen semantischen Unterschied, der, ohne daß Rath selbst diesen Zusammenhang herstellt, konstituiert wird durch die unterschiedliche "Mitteilungsperspektive". 29 Im Falle von a) wäre Negation des Prädikats bei Gïltigkeit des Partizips denkbar, nicht aber bei b): zumindest wäre hier mit Ambiguität zu rechnen.

Dem entspricht es, wenn R. Rüzicka für das Aksl. nicht nach semantischen varianten klassifiziert, sondern nach der Wortstellung. ${ }^{30}$ Präposition der peripherprädikativen Kurzform gegenüber dem Prädikat konfrontiert eher $z$ wei voneinander verschiedene Handlungen und setzt sie zueinander in Beziehung,

29 Rath 71, S. 49, 174

$30 \mathrm{Vgl}$. Rủzickka, SS, S. 56, 65ff, $159 \mathrm{ff}$ 
Postposition tendiert eher zur "synthetischen" Darstellung einer Handlung mit ihrem Merkmal. 31 Die Relevanz des Stellungsgegensatzes erweist sich in der statistischen Verteilung des präs. und des prät. Partizips, 32 in der graduell höheren Prädikativität des präpositiven Partizips und im Gesamtbild der semantischen Varianten. 33

Einschränkungen dieser Grundtendenz der Signifikanz der Wortstellung ergeben sich einerseits für formelhafte und $\mathrm{zu}$ Adverbialstrukturen affine Partizipien in Präposition -dies betrifft allerdings, soweit aus dem Material ersichtlich, nicht den Stellungstyp $\mathrm{P}_{k}+\mathrm{N}+\mathrm{V}-$ und in der Postposition prät. vorzeitige Partizipien und Präsenspartizipien zur Angabe einer geistigen Wahrnehmung als Voraussetzung der Prädikatshandlung. Von den zu Adverbialisierung affinen Belegen abgesehen ist also Präposition eher eindeutig und Postposition ambig.

Ein im Ganzen analoges Bild ergab sich in unserer Magisterarbeit für das aus den Monumenta Serbica gesammelte Material. 34 Hier hatten wir versucht, das sehr vielgestaltige Material, soweit Subjektsidentität und Bezug auf das Prädikat vorlag, auf drei Typen der Konnexion zurückzuführen. Für im Sinne der sprecherintention (und nicht nur syntaktisch) obligate Partizipien 35 ließ sich kein vorherrschender Wortstellungstyp erkennen; bei im Sinne der Sprecherintention fakultativen Partizipien überwiegt bei Angabe eines"Merkmals der Haupthandlung" Postposition, bei "zwei voneinander verschiedenen Handlungen" Präposition.

$31 \mathrm{Vgl}$.a.a.0., s. $85 \mathrm{ff}, 141 \mathrm{ff}$

$32 \mathrm{Vgl}$. a.a.0., S. 84: "In präpositiver stellung wird das präteritale Partizip viel häufiger gebraucht als das präsentische; bei Postposition ist das verhältnis nahezu umgekehrt." ; vgl. auch Vecerka 61, S. $118 \mathrm{ff}, 144 \mathrm{ff}$

$33 \mathrm{Vgl}$. Rư̌izka, SS, S. $136 \mathrm{ff}$

$34 \mathrm{Vgl}$. M.. S. $87 \mathrm{ff}$

35 a.a.O.. S. $44 \mathrm{ff}$ - d.h.: nur bei Mitteilung auch des Partizips ist die Prädikatshandlung die vom Sprecher gemeinte, ohne die Partiziphandlung ist die Aussage im Sinn der Sprecherintention sinnlos. 
7.5 .

Differenzierung der traditionellen Adverbialbestimmung

Die Strukturtypen, die implizit in zusammenhang mit der Signifikanz der Wortstellung und bei der Umschreibung funktionaler Unterschiede unter dem Oberbegriff "sekundäres Prädikat". "doplnék" usw. eine Rolle spielen, werden explizit erörtert in der neueren Adverbialsyntax und -semantik.

Während für Adverbialstrukturen die Notwendigkeit einer strukturellen Differenzierung grundsätzlich anerkannt ist, ${ }^{36}$ macht die systematische Anwendung auf die hochgradig impliziten Gerundial- und Partizipialstrukturen weiterhin Schwierigkeiten. Weiss 77 z.B. vermutet zwar die Notwendigkeit, für Adverbialbestimmungen tiefenstrukturell zu unterscheiden zwischen Satzbezug und Bezug auf das Prädikat, sieht jedoch für die Ableitung poln. Gerundialstrukturen nur eine Ausgangsstruktur vor, die Satzkonnexion durch das "logische und".

Wenn uberhaupt, so besteht Aussicht auf eine systematische Differenzierung sekundärprädikativer Partizipial- und Gerundialstrukturen nicht in Anlehnung an semantische Varianten -hierin ist Weiss beizupflichten-, sondern auf der Grundlage der Unterscheidung von Strukturtypen. Diese Typen sind das Analogon für sekundärprädikative Partizipial-bzw. Gerundialstrukturen und adverbiale Bestimmungen.

Während z.B. Vitek, Steinitz und Ressel aus der lexikalisch expliziten "Variante" der Adverbialstruktur auf Möglichkeiten der Funktion in Typen schließen, sind für die Partizipial- und Gerundialstrukturen umgekehrt nur die Typen der Konnexion der Untersuchung zugänglich und äußerstenfalls Gruppen von mehr oder weniger plausiblen Varianten zuzuordnen.

36 Vgl. z.B. Kŕiźková 66, S. 86: "Scitaem neobchodimym vyjasnit" na kakoj stadi $i$ porozdenija Adv pojavljajutsja, t.e. otnosjatsja li oni $k$ glagol'nomu jadru, ili źe oni predstavljajut soboj skoree rasprostranenie vsego predikata ili daze predloženija v celom.": vgl. Ressel 74, S. 18: "Bei konsequenter Anwendung syntaktischer Kriterien ist es nicht länger möglich. Adv als eine -im syntaktischen Sinne- einheitliche Kategorie aufrecht zu erhalten." 
7.5.1. "Syntaktische" Differenzierung

Ressel $74^{37}$ unterscheidet für das Russische in der Tiefenstruktur zwischen satzorientierten und wortfixierten Adverbialstrukturen; auch die hier angeführten wortfixierten sind syntaktisch fakultativ. Oberflächenkriterium der Satzorientiertheit ist Permutierbarkeit. Vgl.:

My izredka byvaem $v$ teatre: Izredka my byvaem v teatre aber:

Éto dovol'no teploe pal'to: Dovol'no eto teploe pal'to

(Ressel 74.S.31-32)

Die Besonderheiten von Satzadverbien (insbesondere Ersatz durch Proformen, Verhalten bei Negations- und Fragetest) sind begründet durch ihren Kondensatcharakter. 38

Nicht alle permutierbaren Adverbialstrukturen sind notwendig satzorientiert; vgl. die Beispiele bei Ressel, S. 40ff:

$\checkmark$ zooparke on risoval kivotnych: Buduci v zooparke, on risoval kakich-nibud' zivotnych: Gde-to on risoval zivotnych aber:

on risoval zivotnych v zooparke = .. zivotnych, kotorye zivut vooparke

Vgl. auch:

Besedka $v$ sadu postroena nedavno: Besedka postroena nedavo

$v \operatorname{sad} u$

(Fedorov 72, S. 31)

Das heißt: bei Anfangsstellung der lokalen Adverbialbestimmung liegt hier Satzbezug vor, bei Stellung nach dem Nomen adnominale Einbettung. 39

Anders bei Temporaladverbien:

$v$ mae on risoval crety: On risoval cuety $v$ mae

Bei gleicher lexikalischer Bedeutung ist also mit der Möglichket unterschiedlicher Beziehungen $z u$ rechnen. Lokale Adverbial-

$37 \mathrm{Vgl}$. Ressel 74, S. 31ff, 109

38 a.a.o. S. $38 \mathrm{ff}$

$39 \mathrm{Vgl}$. Vitek 67, S. 59, 75ff, 155 
bestimmungen erweisen sich hier als potentiell heterogen, temporale als homogen.

Analog unterscheidet z.B. schon Vitek 67 zwischen russischen Adverbialstrukturen, die sich notwendigerweise nur auf ein Satzglied beziehen und solchen, die sich auf ein Satzglied, z.B. das Prädikat, oder das Satzganze beziehen können. 40 Für Ressel und Vitek steht im Vordergrund die Beschreibung von Möglichkeiten der Funktion von Adverbialstrukturen. nicht die empirische Identifizierung von Funktionstypen im sprachlichen Material, wie sie in unserem Zusammenhang zunächst versucht werden muß, nachdem ja aufgrund einer Partizipial-oder Gerundialstruktur außerhalb des Satzes eine sinnvolle Aussage ubber mögliche Beziehungen und vor allem Einschränkungen hinsichtlich möglicher Beziehungen anders als im Fall der Adverbialstrukturen (s.o. z.B. zu russischen Temporaladverbien gegenüber Lokaladverbien) nicht möglich ist. Vitek 67, s. 62 ist hinsichtlich der empirischen Identifizierbarkeit der definierten Funktionstypen von Adverbialstrukturen skeptisch:

"The decision as to which of the functions is performed by an adverb (or a prepositional phrase) in a specific utterance is often impossible. In such a case the analysis may establish more than one solution, i.e. it will show the construction as ambiguous."

Steinitz schlägt zur Repräsentierung deutscher Adverbialstrukturen folgende Konstituentenstruktur vor: ${ }^{41}$

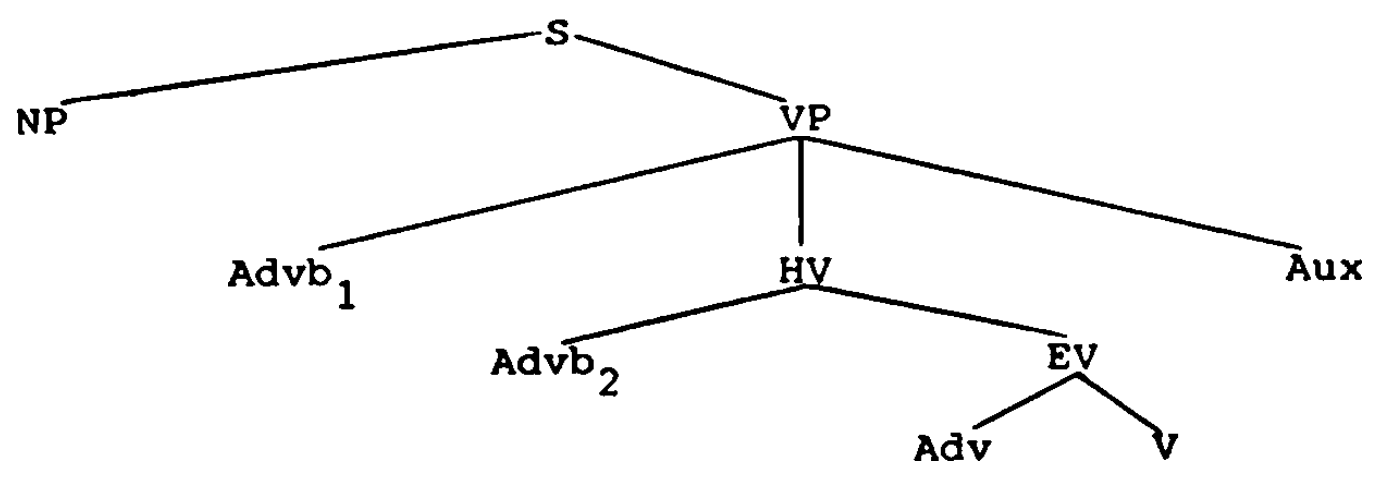

40 Vitek 67 , S. $51 \mathrm{ff}$

41 Vgl. Steinitz 69, S. 31ff, 109; vgl. zu Steinitz Bartsch 72, S. $327 f f$, Bungarten 76 , S. 33ff, 205ff 
Die durch Verbvalenz geforderten Adv, relevant für die strikte Subkategorisierung von $\mathrm{V}$, können in unserem Zusammenhang weitestgehend außer Betracht bleiben, nachem zumindest der Großteil der Partizipial- und Gerundialstrukturen syntaktisch fakultativ ist.

Die fakultativen Advb werden weiter differenziert aufgrund von Beschränkungen bei Kombination von Adverbialen. 42

Es ergibt sich eine provisorische Gliederung nach sieben Gruppen (Varianten), die benannt werden nach ihrer lexikalischen Bedeutung und in zwei Typen auf unterschiedlichen Ableitungsstufen: $A d v b_{1} / A b_{2}$ zusammengefaßt werden. Begründet wird diese syntaktische Differenzierung durch die unterschiedliche Verwendbarkeit, d.h. die Fähigkeit, den Hauptakzent des Satzes zu tragen und Verhalten bei Negation. Dies läßt sich vereinfachend so zusammenfassen, daß $A_{d v b_{2}}$ (unter Bedingungen) den Hauptakzent des Satzes tragen kann und bei Gültigkeit von Advb $_{2}$ Negation des Prädikats nicht möglich ist. 43 Vgl.:

$A_{2} b_{2}:$ Die Kinder spielen im Gárten Die Kinder spielen nicht im Garten 44

aber: Die Kinder spielen im Garten nicht $A_{d v b_{1}}$ : Sie sahen sich ein Jahr lang nicht $A_{d v b}$ ist von der Negation des Prädikats nicht betroffen. $A_{d v b_{2}}$ liegt innerhalb der Reichweite der Negation des Prädikats. Nur Advb, kann $z u$ Adverbialsätzen weiterentwickelt werden. 45 Einzelfragen einmal als geklärt vorausgesetzt, läßt sich hier die Zugehörigkeit $z u A A_{v b}$ oder $A_{1} d v b_{2}$ voraussagen aus der Klassifizierung der Adverbialbestimmungen (nach "Varianten") als kaus.. dur.. temp.. iterativ (Advb $\left.{ }_{1}\right)$ einerseits und andererseits modal, instr. und lokal $\left(\mathrm{Advb}_{2}\right)$.

$42 \mathrm{Vgl}$. Steinitz 69 , S. $47 \mathrm{ff}$

$43 \mathrm{Vgl} . \mathrm{a} . \mathrm{a} .0 . \mathrm{s} .55 \mathrm{ff}$

44 Steinitz geht dabei nicht ein auf Probleme der "Allegation": Die Kinder spielen nicht im Garten ist ambig. Ob die Kinder überhaupt spielen, bleibt hier offen; vgl. hierzu Sgall u.a. 73. S. 75

$45 \mathrm{Vgl}$. Steinitz 69, S. $59 \mathrm{ff}$ 
Die Frage ist, ob sich im Fall der Partizipial- und Gerundialstrukturen aus ihrem Gebrauch Funktionstypen erschließen lassen in Analogie $z u$ der strukturellen Unterscheidung $A_{d v b_{1}}$ : $\mathrm{Advb}_{2} \cdot$

\subsection{2. "Semantische" Differenzierung}

Das Kriterium der Reichweite der Negation, von Steinitz angeführt zur syntaktischen Differenzierung innerhalb des Prädikatskomplexes, ist für Lakoff maßgebliches Argument zur "Herausnahme"von Adverbialbestimungen aus dem Prädikatskomplex und für ihre Formulierung als Prädikat über Sätze. 46 Damit wird die Klassifizierung nach der lexikalischen Bedeutung, bei Steinitz noch relevant für die Beschreibung des syntaktischen Verhaltens, hintangestellt zugunsten der verallgemeinernden Explikation der der Adverbialstruktur implizit zugrundeliegenden Prädikationsbeziehung.

Vgl.:

Ich schlage meine Frau nicht mit Begeisterung

Dieser Satz kann (u.a.) interpretiert werden als

Ich schlage meine Frau (zwar, aber) nicht mit Begeisterung D.h.: negiert ist hier nicht der ganze Satz, sondern nur die Adverbialbestimmung. Deshalb wird dem Satz

Ich schlage meine Frau mit Begeisterung

folgende (vereinfachte) Darstellung mit der Adverbialstruktur als übergeordnetem Prädikat zugeordnet:

$46 \mathrm{Vgl}$. auch Rohrer 71, S. 152: "Wir waren zu dem Schluß gekommen, daß ein Satz auf soviele Teilsätze zurückgeht, wie er Verneinungen zuläßt"; vgl.a.a.0.. S. $152 \mathrm{ff}$ mit Hinweis auf Reichenbach: vgl, auch Immler 74, S. 93ff, 202ff, 209ff: Sgall u.a. 73, S. 77 


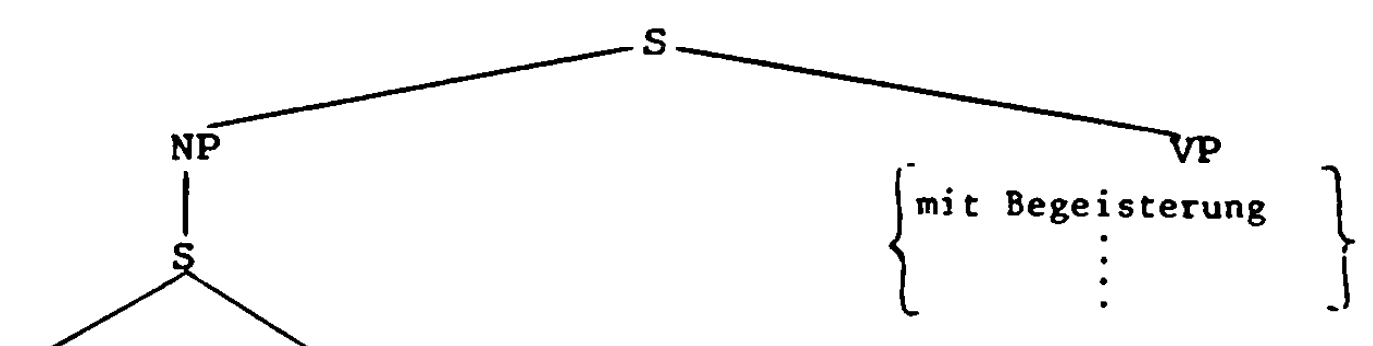

ich schlage

meine Frau

Unsere Aufgabe ist nicht eine modelltheoretische Diskussion: mit dieser Einschränkung sei darauf verwiesen, daß ja analog zur obigen Darstellung von Adverbialstrukturen als Prädikat uiber Sätze z.B. auch Kł̇izková ${ }^{47}$ folgende Konstituentenstruktur anführt für

sofer tri dnja zdal zameny

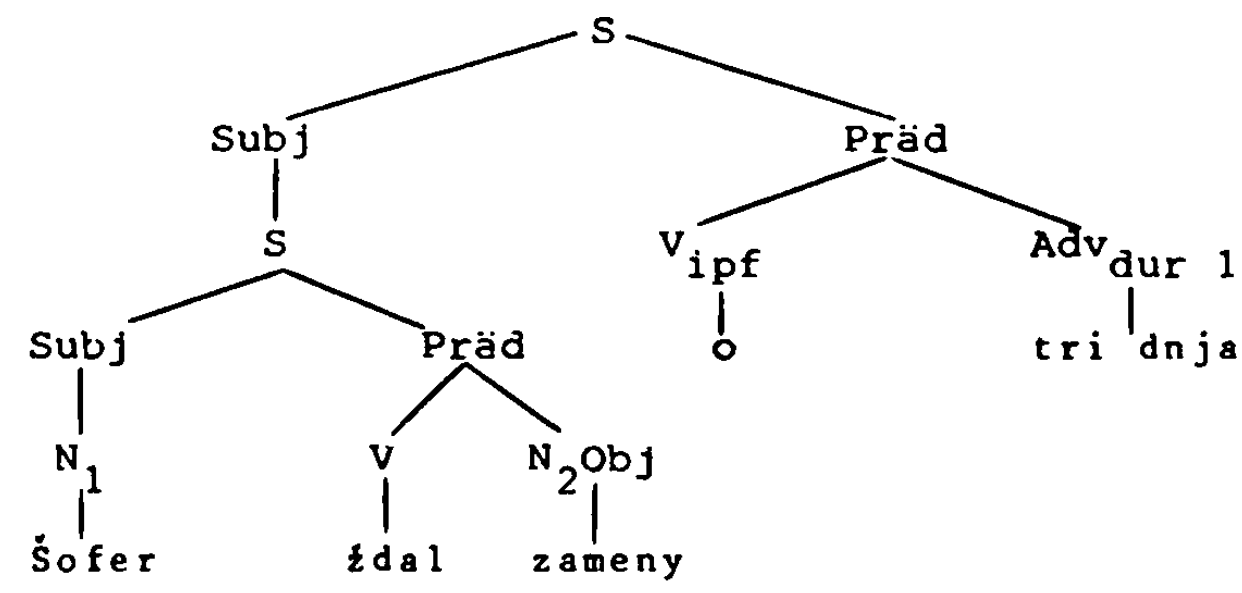

Dem entspricht die Darstellung des Satzdeterminanten bei Ada$\operatorname{mec}^{48}$ :

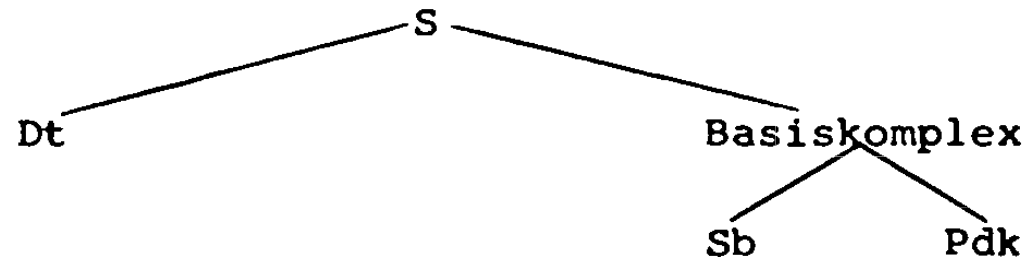

47 Kŕižková in CSR 66, S. 88; dies. 67, s. $78 \mathrm{ff;}$ dies. 68, S. $42 \mathrm{ff}$

48 Adamec in CSR 66, S. 77 
sowie die Darstellung freier Adverbialstrukturen des Deutschen bei Mckay: 49

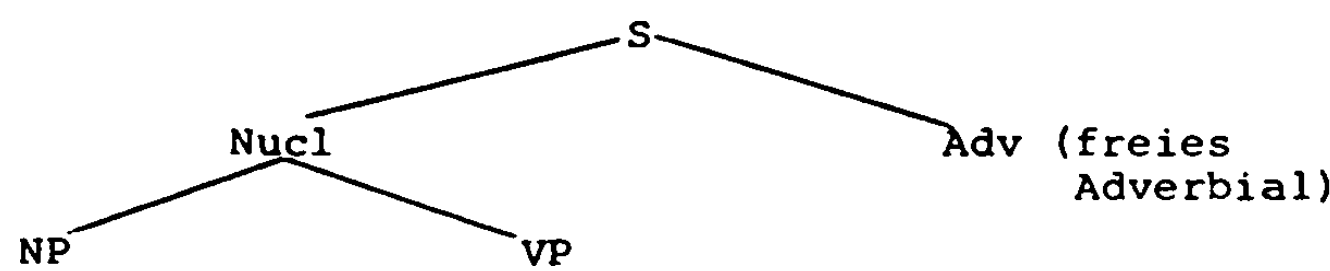

Damit ist der Weg vorgezeichnet, den Bezug von Adverbialstrukturen und so auch sekundärprädikativen Partizipial- und Gerundialstrukturen auf das Satzganze darzustellen, ohne sich zu fixieren auf die lexikalische Semantik der beteiligten Komponenten und eine nicht $z u$ fundierende Analogie von Beziehungsvarianten der "adverbialen" Partizipial- und Gerundialstrukturen zu den lexikalisch expliziten Bedeutungen der Adverbialstrukturen.

Allerdings reicht die undifferenzierte Darstellung anhand einer allgemeinen Relation "Prädikat über Satz" nicht aus zur Repräsentierung evidenter funktionaler Unterschiede, die auch für Partizipial- und Gerundialstrukturen relevant werden. 50 Es muß weiter spezifiziert werden, als Prädikat woruber genau die Adverbialstruktur fungiert. Die Notwendigkeit hierzu ergibt sich nicht nur aus der Möglichkeit der Kombination von Adverbialbestimmungen, die dann zueinander und im Hinblick auf das satzschließende Prädikat hierarchisch anzuordnen sind; vgl.: on echal na gruzovike on medlenno echal na gruzovike on ochotno echal slißkom medlenno na gruzovike

(Ressel 74,5.03)

Es ist von vorneherein die Möglichkeit des unterschiedlichen "Skopus" von Adverbialbestimmungen vorzusehen, auch wenn sie

$49 \mathrm{Vgl}$. hierzu Bartsch 72, S. $318 \mathrm{ff}$

$50 \mathrm{Vgl}$. zur Kritik an Lakoff u.a. Ressel 74, S. 93ff, Bartsch/V. 72, S. 18ff, Bartsch 72, S. 9, 168ff, 315ff: vgl. hierzu Immler 74, S. $164 \mathrm{ff}$ : Sgall u.a.73, S. 188: "In other words, the hierarchy of predicates lor, in linguistic terms, of predications, some of which are "depredicated" while the main clause predication remains with its sentence-forming syntactic value) is semantically relevant." 
alleine auftreten, gerade auch aufgrund des von Lakoff angeführten Kriteriums der Reichweite der Negation. Die undifferenzierte Formulierung der Prädikationsbeziehung uiber einen Satz reicht nicht aus zur Unterscheidung des satzsemantischen Unterschiedes hinsichtlich des Bezugs auf das Satzganze (b) gegenüber dem Bezug nur auf das Prädikat (a) in den folgenden bekannten Beispielen (Bartsch/v.72.S.18ff):

a) Sam sliced the salami carefully (manner adv.)

b) Carefully, Sam sliced the salami (sentence adv.)

Vgl.: Sam carefully sliced the salami (ambig)

Sam carefully sliced the salami carefully

(eindeutig infolge Kombination)

a) ist zu paraphrasieren mit how, b) mit that. Vgl.:

a) Mary sang "America, the beautiful" strangely

b) Strangely Mary sang "America, the beautiful"

$z u$ a): die Art und Weise, wie Mary sang, war eigenartig (Mary's Gesang war eigenartig)

zu b): Die Tatsache, daß Mary sang, war eigenartig.

Zugrunde liegen den unterschiedlichen wortstellungen in a) und b) sowie der Disambiguierung bei Zwischenstellung unterschiedliche Voraussetzungen in der Kommunikation. Die unterschiedlichen Bedingungen der Anwendung sind im Falle ambiger Adverbialstrukturen (s.o.: Zwischenstellung) und jedenfalls bei Partizipial- und Gerundialstrukturen nicht durch die lexikalische Bedeutung $z u$ begründen. Als Kriterium dient u.a. die unterschiedliche Reichweite der Negation, die bei gleichbleibenden Wahrheitsbedingungen und identischem Denotat Auskunft gibt über der Mitteilung zugrundeliegende Voraussetzungen.

Nicht immer betrifft die Negation das Adverb:

Mary didn't sing strangely

Strangely, Mary didn't sing

Strangely, Mary did'nt sing strangely

Nicht bei allen Adverbialbestimmungen ist gleichermaßen Negation des Prädikats möglich; in Gegensatz z.B. zu:

Sie sahen sich ein Jahr lang nicht

(Steinitz,S.57) 
kann das Prädikat nicht negiert werden $z . B$. in den folgenden Sätzen:

- Die Kinder spielen im Garten nicht

(Steinitz,S.57)

- Peter kommt langsam nicht

(Bartsch, S.290)

Petra kocht nicht, wobei sie Salz verwendet

Negiert wird das Verb, nicht die Adverbialbestimmung; diese ist nicht anwendbar bei negiertem Verb, da es in diesem Fall (bei nichtkontrastivem Sprachgebrauch) keinen Bezugsgegenstand für die durch die Adverbialbestimmung dargestellte Prädikation gibt. Möglich ist hier die Negation der Adverbialbestimmung: Die Kinder spielen nicht im Garten (Steinitz,S.57)

Peter iBt nicht mit dem Messer

Vgl. demgegenüber z.B.:

Peter kommt während der Vorstellung

Peter kommt während der Vorstellung nicht

Peter kommt nicht während der Vorstellung

(Bartsch 72,5.291)

Es ist $z u$ unterscheiden zwischen der sprachlichen Bedeutung und dem außersprachlich Bezeichneten (s.o.). So wenig im Rahmen unseres Themas und angesichts des Eorschungsstandes hierzu ausführlich Stellung bezogen werden kann, es soll festgehalten werden, daß sich die sprachliche Bedeutung, wie sie in der Kommunikation übermittelt wird, nicht hinreichend beschreiben läßt anhand der traditionellen an Wahrheitswerten orientierten Logik, vielmehr müssen für die Beschreibung der Satzsemantik auch die Anwendungsbedingungen des Satzes einbezogen werden. Damit wird die Grenze der "traditionellen" generativen Semantik überschritten. Bei gleichem Denotat können Unterschiede hinsichtlich der kommunikativen Voraussetzungen in die satzsemantische struktur eingehen, die unterschiedliche Paraphrasen zur Folge haben und unterschiedliche Möglichkeiten der Negation aufgrund unterschiedlicher hierarchischer zuordnung der impliziten Prädikationen. 51

$51 \mathrm{Vgl}$. Bartsch 72, S. 68ff, 289ff, 293: Sgall u.a.73, S. 75ff "meaning and negation": vgl. a.a.0.. S. $122 \mathrm{ff:}$ "Truth conditions and linguistic meaning" 
Der außersprachlich bezeichnete Sachverhalt ist derselbe in den Sätzen:

The round table is old: The old table is round Unterschiedlich ist ihre sprachliche Bedeutung. Bei identischen Wahrheitsbedingungen kann der Satz: Mein Hund bellt je nach zugrundegelegter Voraussetzung drei verschiedene Negationen haben:

Méin Hund bellt nicht

Nicht méin Hund bellt

Nicht mein Húnd bellt

(Bartsch 72,5.73)

Die Entscheidung über die Reichweite der Negation gibt Auskunft uber die der Mitteilung zugrundeliegenden Voraussetzungen in : John has'nt been here for a month (Immler,s.201) 52 d.h.: a) John war einen Monat lang nicht hier oder: b) John war nicht einen Monat lang hier, er war länger oder kürzer als einen Monat hier

Der Satz

John has been here for a month

steht in Gegensatz zu b), nicht zu a).

Der Begriff der "Präsupposition" selbst soll dabei an dieser stelle nicht problematisiert werden. ${ }^{53}$ Grundlegend ist, daß die Präsupposition außerhalb der Satznegation steht. Der adverbiale Nebensatz als Thema gegenüber dem Restsatz ist präsupponiert und wird nicht negiert durch die Negation des satzschließenden Prädikats. So

z.B. in: Weil er sie liebt, schlägt er seine frau nicht Negation des thematisierten adverbialen Nebensatzes und des Restsatzes ist unabhängig voneinander möglich:

( $N i c h t$ ) weil er sie liebt, schlägt er seine Frau (nicht)

$\mathrm{Vgl}$. demgegenüber:

Er war nicht erfreut darüber, daß sie kamen

52 Vgl. hierzu auch Sgall u.a.73, S. 101

$53 \mathrm{Vgl}$. ausführlich zum Begriff der Präsupposition Sgall u.a. 73, S. 101f, 109, 245ff, ders. in ZfSl 75, S. 784, Bartsch 72, S. 68ff, 73ff, 317ff, Weiss 77, S. 94-95, Anm, 110, Prager Autorengruppe 75, S. $69 \mathrm{ff}, 102 \mathrm{ff}$ 
Hier ist daB sie kamen weder negiert, noch eigentlich behauptet, sondern nur möglich: ${ }^{44}$ daß er sich freut, kann auch andere Ursachen haben.

Anders in:

Darüber, daß sie kamen, war er nicht erfreut

(Prager Autorengruppe.S.72)

Hier wird infolge Thematisierung des Nebensatzes sein Inhalt präsupponiert, es wird vorausgesetzt, daß sie kamen eingetreten ist.

Damit ist verwiesen auf die konstituierende Relevanz der Sprecherintention für die Organisation der den Oberflächenstrukturen von Adverbial- sowie auch Partizipial- und Gerundialstrukturen zugrundeliegenden semantischen Beziehungen als strukturen der in der Kommunikation mitgeteilten sprachlichen Bedeutung.

Für die lexikalisch expliziten Adverbialbestimmungen ergeben sich dabei Einschränkungen hinsichtlich ihrer Verwendbarkeit, bzw.: Möglichkeiten des unterschiedlichen Verhaltens hinsichtlich 2.B. der Negation sind aus diesen selbst vorhersagbar - anders bei den Partizipial- und Gerundialstrukturen. Für sie darf weitestgehende Verfügbarkeit für die Sprecherstrategie erwartet werden.

Das heißt: Es ist nicht möglich, wenn wir versuchen, Typen von Beziehungen, wie sie formuliert wurden in Zusammenhang mit Adverbialstrukturen, anzuwenden auf Partizipial- und Gerundialstrukturen, eine vorgeblich syntaktische Differenzierung des Prädikatskomplexes als identifizierbar vorauszusetzen und dann unterschiedliche Möglichkeiten der Realisierung dieser syntaktischen positionen in der aktualen Gliederung zu erörtern, wie dies z.B. Kaćala für doplnok-Strukturen 55 oder für Adverbialstrukturen Adamec $66^{56}$, Uhlirova ${ }^{57}$ oder Pala ${ }^{58}$ tun. Allenfalls

54 Zur "Allegation" vgl. Prager Autorengruppe 75, S. 72ff, Sgall in ZfSl 75, S. 785, ders. u.a.73, S. $108 \mathrm{ff}$

$55 \mathrm{Vgl.Kacala} 71$, S. $102 \mathrm{ff}$

56 Adamec 66, P.S.

57 Uhlírová in Daneš 74 (ed.). S. $208 \mathrm{ff}$

58 Pala in Daneš 74 (ed.), S. $196 \mathrm{ff}$ 
kann so das oberflächensyntaktische Ergebnis der Konstituierung von Funktionstypen auf der Ebene der sprachlichen Bedeutung benannt werden.

7.6 . Arwendung auf Partizipial- und Gerundialstrukturen

Für die Anwendung neuerer Ergebnisse der Adverbialsyntax und -semantik auf strukturen mit impliziter subjektsidentität sind exemplarisch die Ansätze von Bartsch 72, Weiss 77 und Rúžiłka 66.

Bei Bartsch 72 ist für unsere Zwecke vorrangig die Unterscheidung von "relationalen" und "modalen" Adverbialen: letztere sagen etwas aus über einen Handlungsvollzug oder -ablauf (Vorgang). 59 während relationale Adverbiale auf der Konnexion von Sätzen beruhen und Ereignisse oder Umstände zueinander in Beziehung setzen: die sprachliche Form der Konnexion hängt ab von Kontext und situation. 60

Auf der Grundlage der Satzkonnexion werden auch "Attributionen zu Satzsubjekt in adverbialer Position" beschreiben, bei denen das subjekt der durch das Konnektiv verbundenen Sätze referenzidentisch ist. 61

Vgl.:

1) Er schimpt wutentbrannt

Er ramm um die Ecke biegend einen PKW

2) Stillschweigend nahm sie an der Prüfung teil

zögernd ging sie zur Demonstration Anders als bei modalen Strukturen ist für die Belege unter 1) und 2) folgende Paraphrase nicht möglich:

Er redet leichtsinnig ("mod.") / Sein Reden ist leichtsinnig/ Sein leichtsinniges Reden

Im Unterschied zu modalen Adverbialen ist möglich:

59 Bartsch 72, s. $16 \mathrm{ff}$

60 a.a.o.. S. $105 \mathrm{ff}$

61 Vgl.a.a.O.. S. $140 f f$ "Attributionen zum Satzsubjekt oder Akkusativobjekt in adverbialer Position" 
Daß sie an der Prifung teilnimmt, geschieht mit Bedauern (bedauernd, mit zögern, zögernd)

Nach Bartsch 72, S. 141 auch:

Um die Ecke biegend geschieht es, daß er einen PKW rammt Die bisher angeführten Paraphrasen unterscheiden zunächsteinmal strukturen mit referenzidentischem subjekt gegenuber solchen (modalen), für die kein identisches subjekt $z u$ erschlieBen ist. Nun ist aber auch innerhalb der Belege mit identischem Subjekt unterschiedliches Verhalten bei der Negation festzustellen. 62

Vgl. in 1): Er schimpft wutentbrannt nicht (Negation des V) Er schimpft nicht wutentbrant (Negation des Adv.) Vgl. in 2): Sie nahm nicht mit Bedauern an der Prïfung teil (Negation des Adv.) Sie nahm mit Bedauern nicht an der Prïfung teil (Negation des $V$ )

D.h.: bei 1) ist nur die Negation von Adv. möglich, das Adv. setzt das Prädikat voraus: ${ }^{63}$ dieses kann nicht alleine negiert werden (vgl. "modale" Adv.). In 2) können Adv. und Prädikat unabhängig voneinander negiert werden ("relational"). vgl. "modal"64:

- Peter kommt langsam nicht

(Bartsch 72.5 .290$)$

Dem unterschiedlichen Verhalten hinsichtlich der Negation des Prädikats bei relationalen und modalen Adverbialbestimungen entspricht bei strukturen mit subjektsidentität unterschiedlicher Gebrauch von Konfunktionen: 65

$62 \mathrm{Vgl}$. Bartsch 72, S. $143 \mathrm{ff}$; Bartsch' Ausfürungen sind hier verwirrend genug, vgl. S. 24 zur Definition der Testverfahren: "(36) Möglichkeit der Negation vor dem Adverbial (d.h.. auf das Adverbial bezogen) in nichtkontrastiven Außerungen (37) Möglichkeit der Negation nach dem Adverbial (d.h.. auf das Verb bezogen) in nichtkontrastiven Äußerungen"; demgegenuber S. 144: "Test (36) verläuft positiv - d.h.. das verb ist negierbar...": auf S. 143 wird als Test (37) angegeben die stellung vor dem adverbialen Attribut und, steht $z u$ vermuten, als Test (36) die Stellung danach!

$63 \mathrm{Vgl}$. a.a.O.. S. $289 \mathrm{ff}$

$64 \mathrm{Vgl}$. a.a.O.. S. 146ff, S. 172: "Bei den modalen Adverbialkonstruktionen wird das Adverb direkt auf das Prädikat angewandt.",

65 a.a.o.. S. $173 \mathrm{ff}$; Filipović $77 \mathrm{zu}$ "temp I, temp II, mod" 
beim oben angeführten Typ 2)

sind Paraphrasen möglich mit während, bevor, nachdem, weil, trotz usw.

beim oben angeführten Typ 1)

(Negation des Prädikats nicht möqlich) sind Paraphrasen mit wobei, wozu denkbar:

Er schimpt, wobei er wutentbrannt ist

- Er schimpft nicht, wobei er wutentbrannt ist

Petra kocht, wobei sie Salz verwendet

- Petra kocht nicht, wobei sie Salz verwendet

aber:

Er schimpte nicht, während er wutentbrannt ist

Kurz: die von Bartsch angeführte Unterscheidung relationaler gegenüber modalen Adverbialstrukturen findet ihre Analogie innerhalb der Strukturen mit Subjektsidentität; zur Abgrenzung der relationalen gegenüber den modalen Strukturen im weiteren Sinne genügt der Hinweis auf Subjektsidentität nicht. Er sagt noch nichts aus uiber das Verhalten bei Negation und die hiermit verknüpften strukturellen Unterschiede hinsichtlich Bezug auf das Satzganze : Bezug auf das Prädikat.

Weiss klassifiziert poln. Gerundialstrukturen als Adverbiale mit den zusätzlichen Merkmalen der untergeordneten Prädikativität und der Subjektsidentität. ${ }^{66}$

Für sämtliche Gerundialstrukturen sowie die nichtrestriktiven attributiven Partizipialstrukturen gilt als gemeinsamer Ausgangspunkt der transformationellen Ableitung die tiefenstrukturelle verknüpfung zweier Sätze durch das "logische und". 67 Diese allgemeine Satzkonnexion wird auch zugrundegelegt für Partizipien im Rahmen des Prädikatskomplexes, die üblicherweise als Prädikatsnomen beschreiben werden. 68

vgl. Weiss 77, S. 341 :

66 Weiss 77 , S. $272 \mathrm{ff}$

67 a.a.O.. S. $338 \mathrm{ff}$

68 a.a.o.. s. 362 


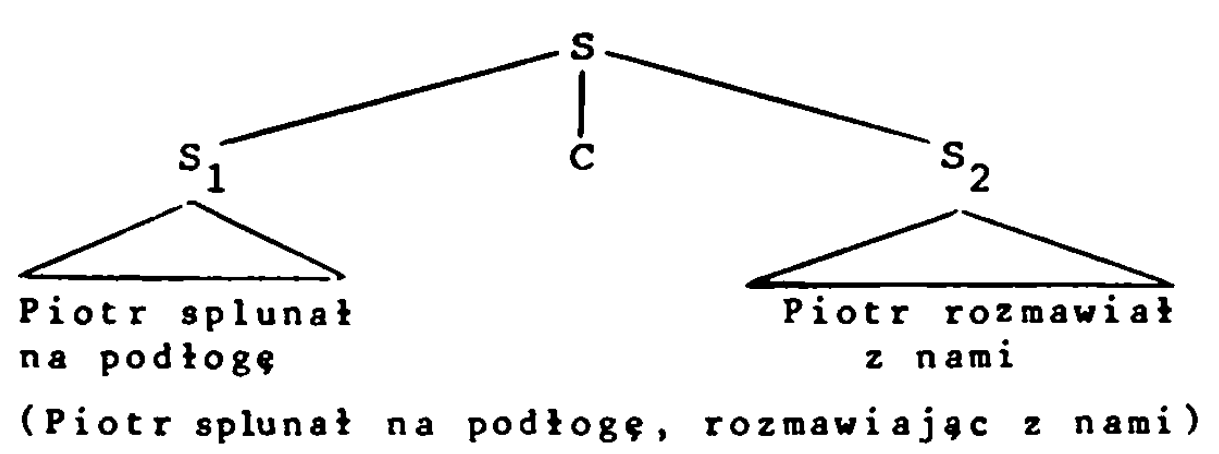

Demgegenuber sind restriktive Attribute das Ergebnis einer adnominalen satzeinbettung.

Motiviert ist diese ökonomische zusammenfassende Ableitung von der Satzkonnexion durch die kommunikative Funktion der untergeordneten Prädikativität im Unterschied zur Nichtprädikativität der restriktiven Attribute ${ }^{69}$ sowie durch die ausfuhrlich dargestellten Schwierigkeiten einer systematischen Differenzierung des Prädikatskomplexes. Die Aussichtlosigkeit einer "Klassifizierung" anhand der durch Paraphrasen gewonnenen semantischen "Varianten" wurde bereits dargelegt. 70 Beizupflichten ist Weiss auch, wenn er die Frage "Zwei Typen von Part-IIKonstruktionen?" 71 verneint, soweit die Differenzierung von Funktionstypen der Gerundien begrundet wird aus syntaktischsemantischen Eigenschaften der Gerundien selbst bzw. der zugrundeliegenden Verben, konkret durch die Nichterweiterung des Gerund und durch die Unmöglichkeit der Paraphrasierung infolge "Hyponymie". 72

Hieraus folgt aber noch nicht notwendig die Unmöglichkeit, in der Tiefenstruktur Typen der Konnexion $\mathrm{zu}$ unterscheiden, wie sie Weiss selbst für Adverbialstrukturen zumindest vermutet, 73 nicht aber für Gerundialstrukturen, denen er doch auch adverbialen Charakter zuschreibt.

$69 \mathrm{Vgl}$. Weiss $77, \mathrm{~s}, 367,384,388$

70 a.a.o.. s. $315 \mathrm{ff} ;$ ders. 74 , S. $193 \mathrm{ff}, 325 \mathrm{ff}$

71 Weiss 77 , S. $275 \mathrm{ff}$

72 a.a.o.. s. 276 : on szedz kulejoc

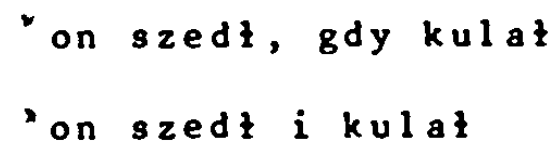

73 a.a.0.. s. 35, $272 \mathrm{ff}$ 
Wurde Lakoff aufgrund der Undifferenziertheit der Repräsentierung von Adverbialstrukturen als Prädikat über Sätze kritisiert, so ist zweifelhaft, ob die analog undifferenzierte Satzkonnexion durch das "logische und" bei weiss ausreicht zur spezifizierung evidenter funktionaler Unterschiede.

Weiss selbst repräsentiert die Unterschiede zwischen den durch die allgemeine Satzverknuipfungsrelation dargestellten strukturen anhand von Transformationen ${ }^{74}$ auf dem Weg $z$ ur oberflächensyntaktischen Repräsentation. Dies betrifft einerseits die Zuordnung nichtrestriktiver strukturen zum subjekts- oder Prädikatskomplex und andererseits innerhalb des Prädikatskomplexes die Unterscheidung von interner und externer peripherer Prädikation und in Zusammenhang hiermit die Unterscheidung der Funktion als Kulisse oder Fokus. ${ }^{75}$ Der Status der funktionalen Unterschiede insbesondere innerhalb des Prädikatskomplexes bleibt problematisch, es ist fraglich, ob Unterschiede, die im Fall der Adverbialstrukturen der Tiefenstruktur zugeordnet werden, zumindest in unmittelbare Nähe der oberfläche zu rücken sind, die ja ihrerseits nicht eindeutig ist.

Die Satzkonnexion durch das "logische und" 76 ist ihrer Herkunft nach eine elementare prädikatenlogische und kommunikativ indifferente Relation. So, wie sie von Weiss verwendet wird, enthält sie zugleich kommunikativ-semantische Implikationen, insofern sie den Gegensatz restriktiv : nichtrestriktiv und, hiermit verknüpft, den Gegensatz zwischen kommunikativ, nicht logisch verstandener untergeordneter Prädikativität und Referenzakt bei restriktivem Attribut (das durch adnominale Einbettung dargestellt ist) repräsentiert.

Der kommunikative Unterschied zwischen Referenzakt und untergeordnet prädikativer Funktion wird auch bei Weiss relevant für die Stellung in der aktualen Gliederung: Nichtrestriktive Attribute können nicht fokussiert auftreten (z.B. als Erweiterung

74 Weiss 77, S. $338 \mathrm{ff}, \mathrm{vgl}$. auch S. 367,384

75 a.a.o., s. $300 \mathrm{ff}, 377$

76 a.a.o.. S. $325 \mathrm{ff}$ : Rohrer 71, S. 187 
zum Prädikatsnomen), restriktive Attribute sind fokussierbar.

Weiss' Differenzierungen des Prädikatskomplexes hinsichtlich interner/externer Prädikation und hinsichtlich der Modalität von Gerundialstrukturen, die ihren Reflex findet in der Wortstellung und im Verhalten bei Negations- und Fragetest, verweisen ebenfalls auf die Ebene der in der Kommunikation konstituierten sprachlichen Bedeutung. Wenn fokussierte strukturen in der Tiefenstruktur einen Sonderstatus haben, 77 soweit sie an der oberfläche auftreten als restriktive Attribute, ist zu fragen, ob nicht auch die Fokussierung im Rahmen des oberflächensyntaktischen Prädikatskomplexes ${ }^{78}$ ihren tiefenstrukturellen Reflex finden sollte.

Die Alternative wäre U.E., entweder das "logische und" freizuhalten von kommunikativ-semantischen Assoziationen und damit restriktive und nichtrestriktive strukturen in einer prädikatenlogischen Darstellung nicht zu unterscheiden-dies wirde den sprachlichen Tatsachen nicht gerecht-;oder aber analog zur Differenzierung des oberflächensyntaktischen Nomi nalkomplexes aufgrund der tiefenstrukturellen Unterscheidung restriktiver und nichtrestriktiver Strukturen auch Gerundien im oberflächensyntaktischen Prädikatskomplex zu differenzieren auf der Grundlage von kommunikativ-semantischen Beziehungen, auf die ja auch die Paraphrasen bei Weiss in Zusammenhang mit dem Unterschied Kulisse : Fokus hinführen.

Soweit uberhaupt Aussicht besteht auf eine konsistente Differenzierung des Prädikatskomplexes hinsichtlich des Gegensatzes Bezug auf das Satzganze : Bezug auf das Prädikat, den auch Weiss für Adverbialstrukturen vermutet, für Gerundialstrukturen aber nicht diskutiert, betrifft dies die Ebene der sprachlichen Bedeutung.

Zugrunde liegt letztlich die ungelöste Problematik des Begriffs der Tiefenstruktur und der stellung der Ebene der sprachlichen

$77 \mathrm{Vgl}$. Weiss $77, \mathrm{~S} \cdot 367$

78 a.a.o.. S. $361 \mathrm{ff}$ 
Bedeutung in einer zu modifizierenden Tiefenstruktur. Unter Einbeziehung der Sprecherintention ist eine analoge Differenzierung für Partizipial- und Gerundialstrukturen zu motivieren.

Gegenüber Weiss und Kłizková sieht Razicka 66 nicht einen gemeinsamen Ausgangspunkt für die Ableitung sämtlicher untergeordnet prädikativer strukturen vor, sondern unterscheidet von vorneherein zwischen restriktiven und nichtrestriktiven Strukturen im Rahmen des subjektskomplexes und untergeordnet prädikativen Partizipien und Gerundien im Rahmen des Prädikatskomplexes mit Subjektsidentität. Während Kr̉iźková 69 zur strukturierung des Prädikatskomplexes zurückgreift auf das oberflächenkriterium der Isolierung (zur Unterscheidung von Prädsec und AdvComit) und Weiss zugunsten der allgemeinen Satzverknüpfung auf eine tiefenstrukturelle Differenzierung der Funktionstypen, die dann oberflächensyntaktisch im Prädikatskomplex auftreten, verzichtet, unterscheidet Rủzicka "sekundäres Prädikat" und im Rahmen des primären Prädikatskomplexes "Advb". Es wird (abgekürzt) folgende Konstituentenstruktur angenommen:

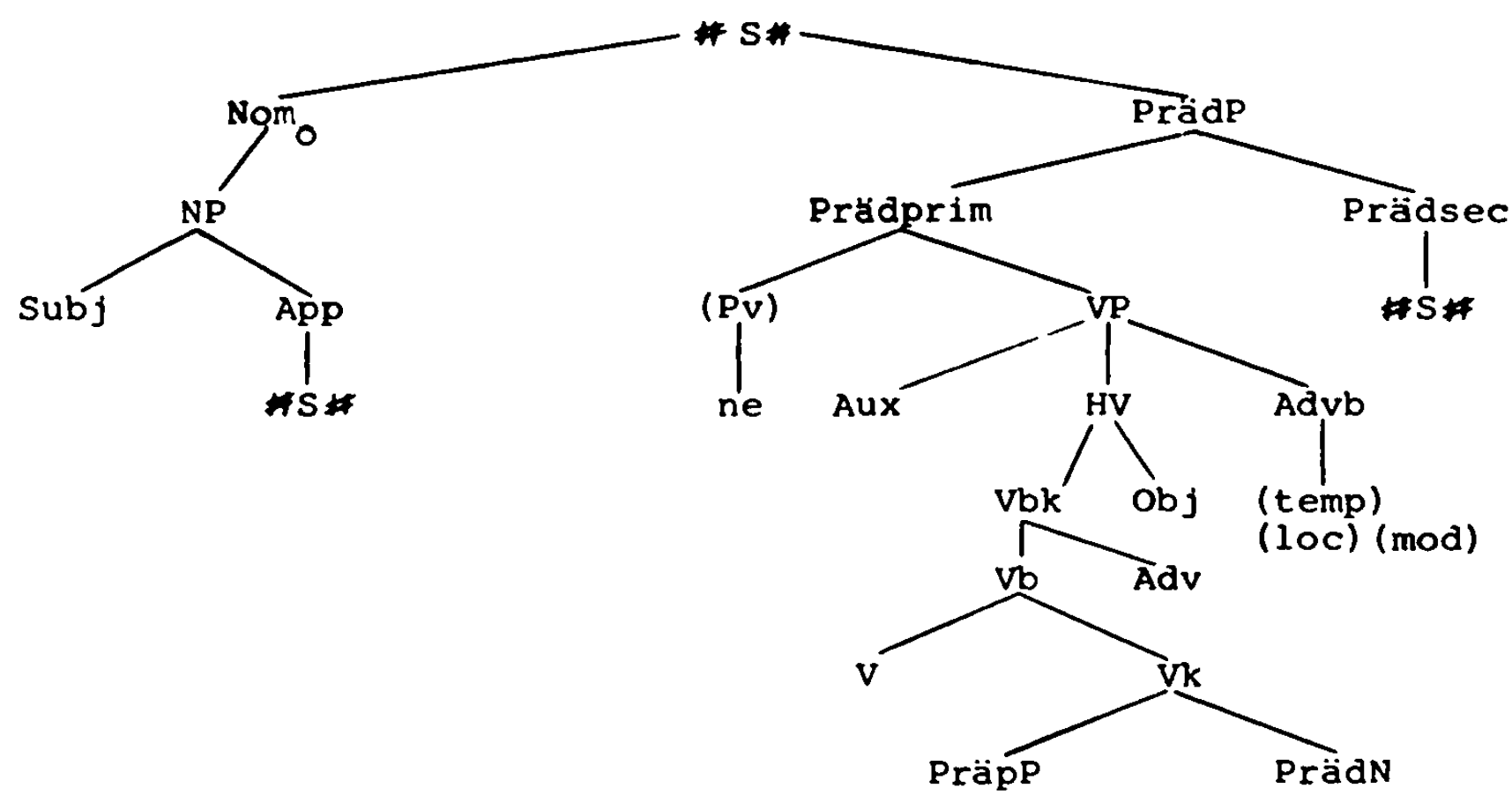


Rủicka selbst betont die Vorläufigkeit dieser Unterscheidungen: ${ }^{79}$ sie wird mehr veranschaulicht durch Beispiele und Hinweis auf Oberflächenkriterien als definiert. Entscheidend in unserem zusammenhang ist aber doch, daB ansatzweise nicht mehr nach durch Paraphrasierung gewonnenen Varianten, sondern nach Strukturtypen spezifiziert wird. Für Prädsec im Unterschied zu Advb wird Stellung außerhalb der Reichweite der Negation des Prädikats angenommen. Die Konstituentenstruktur legt für das sekundäre Prädikat eher koordinative Beziehung zum primären Prädikat als Bezug auf das Satzganze nahe. An Oberflächenkriterien zeichnen sich ab obligate Isolierung und bevorzugte Anfangsstellung für Prädsec, für Advb bevorzugte Endstellung. nicht immer obligate Isolierung sowie möglicherweise unterschiedliche Realisierung der Korrelation (Prädsec : Part/Ger; Advb : Ger?)

Vgl. z.B.:

Prädsec:

podavlennaja blagodarnost'ju, Ann-Mari v bezuprecnom obchozdenii ugadyvala chulu (Rủžicka 66,S.41) poglošaja glagol, pogložennyj obrazami, ja ucelel tol'koblagodarja nesovmestimosti dvuch opasnostej

odnako Sarl'predpolel udrat' iz doma, pustivłis' vdogonkuza cirkovnoj naezdnicej

(on) sdelalsja priverzencem prjamogo metoda, ob-javiv sebja vposledstrii ego osnovopoloznikom

(Rủzið̌ka 66, S.45)

In den folgenden Beispielen wird die isolierte struktur in Anfangsstellung über Prädsec, die Gerundialstruktur in Endstellung über Advb im primären Prädikatskomplex abgeleitet:

Napevnye, zvucnye.. oni upivalis'sami soboj..., nimalo ne zabotjas' obo mne

(Rưzicka 66, S.50,vgl. 58 Prostivsis'so vsemi, on ußgel ulybajas' (Rủzicka 65, Verbalphr., S.312)

Es ist am Material zu überprüfen, ob sich hier die vorgeschlagene Gliederung des Prädikatskomplexes systematisieren läßt unter

79 Rúzicka 66, S.42, 55; ders. 65, Verbalphr., S. 352: "Ausreichend präzise Unterscheidungskriterien sind noch nicht beizubringen" 
Einbeziehung der Relevanz der Sprecherintention. Weiss' Satzverknüpfungsmodell hat natürlich den Vorzug, generell zu sein, allerdings um den Preis, daß evidente funktionale Unterschiede keinen rechten Platz im System finden.

Die Form der Darstellung soll so gewählt werden, daß sie die Reichweite der durch die Partizipial-/Gerundialstruktur geleisteten Prädikation über das Satzganze verdeutlicht. Dieser Satzbezug gegenuiber Bezug auf das Prädikat kann solange nicht gut dargestellt werden, als man das binäre Schema $S \rightarrow$ Subjph.r. + Prädphr. zugrundelegt und jeweils nur dem Subjekts- oder Prädikatskomplex einseitig untergeordnete Erweiterungen innerhalb des Satzes als Nebensatzglieder in verschiedener. paradigmatisch durcheinander ersetzbaren Formen vorsieht. Diese Problematik liegt den (syntaktischen) Konstituentenstrukturen z.B. von Steinitz, Miko, Rúżička, Kŕižková oder Kade zugrunde, auch wenn z.B. Steinitz und Rủzicḱa innerhalb des Prädikatskomplexes nach Positionen differenzieren.

Einzelheiten der transformationellen Ableitung sollen im Rahmen unserer Darstellung nicht im Vordergrund stehen. Hierzu liegen umfangreiche Arbeiten vor, so in letzter zeit, insbesondere zur Struktur der Nominalphrase, die Untersuchung von Weiss 77 . Schwerpunkt unserer Uberlegungen ist nicht die Generierung untergeordnet prädikativer Strukturen, sondern zunächst einmal die Motivierung der Unterscheidung von Funktionstypen am sprachlichen Material, die Frage nach der Konstituierung für die Spezifizierung relevanter Kategorien, die U.E. in einer modifizierten "Tiefenstruktur" ihren Platz finden müßten; dies wäre Bedingung für eine konsistente Generierung.

Bei allen ungelösten methodologischen Problemen gehen wir aus von der grundlegenden Relevanz der kommunikativen Intention des Sprechers für die Konstituierung von Funktionstypen der Partizipial- und Gerundialstrukturen. Deren Stellung in der aktualen Gliederung soll in einer notwendig höchst provisorischen weise schematisch dargestellt werden, ${ }^{80}$ durch Formulierung von Grund-

$80 \mathrm{Vgl}$. Dezsö/Szépe in Dahl 74 (ed.), S. 70: "We could find ... 
typen der aktualen Gliederung und deren zuordnung $\mathbf{z u}$ Oberflächenstrukturen.

Die Form dieses Schematismus ${ }^{81}$ lehnt sich an an Dezsö/Szépe. Adamec und Danes. Als grundlegend wird die binäre Gliederung von $\Sigma$, der Ausgangsgröße zur Symbolisierung der gesamten Kommunikationseinheit, in Thema und Rhema ( $T: R)$ angenommen sowie die Möglichkeit, im Falle komplexerer Einheiten die primären $T_{1}$ und $R_{1}$ ihrerseits binär und stufenweise $z u$ zerlegen.

Vgl. Dezsö/Szépe in Dahl 74 (ed.), S. 70ff:

"Our starting point was that the relation of the topic and
the comment is strictly dyadic (this dyadicity is of a
theoretical character): in a communication unit (that is
in the equivalent of a grammatical sentence) the minimal
demand is one comment: in two-member sentences generally
one topic part and its comment part.
Then we supposed -on an entirely formal basis- that if
either the topic part or the comment part can be devided
into two further parts (consists of two members) one of
them can be considered a secondary topic, the other one
a secondary comment. Also in this case we insisted on
dyadicity, on principle it is not necessary, in technical
respect, however. it does more good than harm.
After the secondary topic-comment relation we assumed
the tertiary and quartiary case as well."

80 ... no elaborate system describing the depth of the communicative pattern (topic-comment relation), we had to outline a possible system ourselves."; vgl. auch Weiss 77, s. $49 \mathrm{ff}$

81 Dezsö/Szépe in Dahl 74 (ed.), S. 65ff; dies. in Danes 74 (ed.). S. 81ff: Petöfi 71, S. 200ff: Adamec in Danes 74 (ed.). S. 189ff: vgl. hierzu auch Weiss 77, s. 50ff: vgl. Daneš in Danes 74 (ed.), S. $106 \mathrm{ff}$ 
8.

Funktionale Differenzierung des

Prädikatskomplexes - sprachliches Material

8.1. Anfangsstellung

8.1.1. Russ. Ger $+\mathrm{I}+\mathrm{N}+\mathrm{V}$

In dem von Jacobsson ${ }^{1}$ untersuchten Material stehen Gerundien annähernd zweimal so oft vor dem Prädikat als danach: Erweiterung des Gerund reduziert die statistische wahrscheinlichkeit der Voranstellung. Bei dem von Valimova untersuchten Material 2 steht gleichzeitiges Gerund in ca. zwei Dritteln der Belege nach dem Prädikat, vorzeitiges Gerund in ca. zwei Dritteln vor dem Prädikat.

Insbesondere ist bei pf. Gerundien in der Bedeutung der Nichtvorzeitigkeit Nachstellung weitgehend verbindlich und Voranstellung relevant für die Signalisierung der vorzeitigkeit. 3 Für die folgenden präsentischen und präteritalen pf. Gerundien in Anfangsstellung nehmen wir als Grundbedeutung hinsichtlich der Zeitenfolge Reihenfolge zwischen der Handlung des Gerund und des Prädikats an. Als Variante ist u.a. vorzeitig-resultative Bedeutung denkbar, d.h. Gültigkeit des Ergebnisses der Nebenhandlung für die zeit der Haupthandlung.

Vgl. z.B.:

Napisav pis'mo, ja otdychal (otdychaju, obytno otdychaju, budu otdychat') (Leitfaden, S.147) Protitav (protitaja) knigu, on zadumalsja (Isatenko 75,5.3324 Uechav iz doma, ja nacal samostojatel'no zit'verorode (sint.r.j.74,S.138) Zur Möglickkejt zusätzlicher semantischer Nuancen vgl. z.B.: Uslyav strel'bu na ulice, Anton podosel k oknu = kogda ...

1 Jacobsson, Gö. 69, S. 44, $140 \mathrm{ff}$

2 a.a.o., s. 54

3 Vgl. Rủzička 66, S. 47ff; Kłižková 69, Synt.ch., S. 121ff; sint. r. j. 74, S. 147

4 Zum möglichen Bedeutungsunterschied zwischen präs. und prät. pf. Gerund vgl. Isakenko 75, S. 332 mit Hinweis auf R.Jakobson 
potomu cto..., chotja...

(Babby 75, S.5)

Für Gerundialkonstruktionen mit konzessiver Semantik zeichnet sich die Möglichkeit (nicht die Notwendigkeit) ab, daß die Variante der semantischen Beziehung schon bei der kondensierten struktur selbst expliziert wird und damit als vom sprecher intendiert bezeichnet ist, während sonst (s.o.) die Angabe von Varianten notwendig subjektiv ist und grundsätzlich nicht davon ausgegangen werden kann, daß uberhaupt eine derartige Spezifizierung, wie sie durch die ublichen Paraphrasen suggeriert wird, intendiert ist.

Vgl. z.B. mit Explikation der konzessiven Relation:

Daze priloziv vse svoi sily i upotrebiv ves' svoj um, on ne smog dobit'sja postavlennoj celi

(Adamec II 75, S.177)

Do nitki promoknuv pod dozdem, ochotnik vse-taki ne vernulsja domoj = Chotja ochotnik do nitki promok pod dozdem, on vsetaki ne vernulsja domoj

(Mulisch 75, S.207)

Ispytav na sebe ich plodotvornoe vijanie, oni vmeste s tem ne byli rabami ich filisofiteskich sistem, a trorteski obladev ich peredovymi idejami, razvivali materializm dal'se

(Kade 68,S.610)

Für die folgenden Beispiele mag konditionale Interpretation naheliegen. In diesem Fall ist Paraphrase durch Koordination nicht möglich. 5 Gleichzeitig ist $z$ u betonen, daß für die iberwiegende Zahl der hier eingeordneten Belege auch andere Interpretationen nicht auszuschlieben sind, so z.B. temporale, kausale, instrumentale (nachdem, weil, dadurch daß).

Tol'ko izuciv do tonkostej mechanizm razvitijabolezni, my smožem vlijat' na nego

(Adamec II 75, S. 176)

Posmotrev v mikroskop na kaplju tistoj vody, vy uvidite v nej mozestro malen'kich zivych suscestv (Sint.r.j.74,5.137)

Bei ipf. Gerundien dieses stellungstyps ergibt sich grundsätzlich hinsichtlich der semantischen Varianten und hinsichtlich der $z u$ erörternden Typen der Konnexion ein analoges Bild $z u$ den pf. Beispielen, diesmal auf der Grundlage der Bedeutung der

$5 \mathrm{Vgl}$. Bartsch 72, S. $106 \mathrm{ff}$ 
Gleichzeitigkeit. Weit überwiegend treten ipf. Formen im Präsens auf, es finden sich aber auch einige präteritale Formen. Z.B.:

Sidja za stolom, ja Cital/Citaju/budu Citat' knigu

(Leitfaden, S.147)

Razrabatyvaja kontrol'nye cifry na period general'noj perspektivy, my nametaem... (Kade $68,5.610)^{6}$

Dumaja o svoem pokolenii, ja vsegda vspominaju te dalekie dni (Adamec II 75, S.178)

Explikation der konzessiven Beziehung:

Rabotaja desjat' tasov v den', on vse-taki uspeval sledit'za literaturnymi novinkami

(Adamec II 75, S.177)

Ne ozidaja nitego chorosego ot ètoj poezdki, ja vse że poechal vmeste so vsemi = Chotja ja ne ozidal nitego chorosego ot étoj poezdki, ja vae ze poechal

(sint.r.j. $74,5.138)$

(u.a.) konditional:

Znaja kod, my mozem prekrascat' zakodirovannuju rabotu avtomatov = esli my znaem kod, my mozem..(Adamec II 75, S.178) Zanimajas' ser'ezno, ty mozes' dobit'sja chorosich rezul'tatov = Esli ty ser'ezno zanimaes'sja, ty mozes'dobit' chorosich rezul'tatov (Mulisch 75.5.207)

ipf. präteritales Gerund:

Ne imev/imeja druzej, on vel otsel'niceskij obraz zizni

(Isatenko $75, \mathrm{~S} .328 \mathrm{ff}$ )

Da $B$ die hier zugrundegelegte Auffassung der Gleichzeitigkeit ipf. Gerundien der Präzisierung bedürfte, ${ }^{7}$ zeigen folgende Beispiele, gleichzeitig aber auch, daß bei unterschiedlicher Zeitenfolge der semantische strukturtyp unverändert bleibt. Vgl. ipf. präs. Gerund zur Angabe der regelmäßigen zeitlichen Aufeinanderfolge:

Vstavaja na rassvete, ona spuskalas' v kuchnju i vmeste s kucharkoj gotovila zakusku k caju (Mulisch 75,S.203)

6 Nicht einzusehen ist, warum für diesen Strukturtyp gemäß Kade 68, S. 610 ausschließlich Paraphrase/Nbersetzung durch Präpositionalkonstruktion möglich sein soll, nicht aber temporaler Nebensatz.

$7 \mathrm{Vgl}$. 2.B. auch Isatenko 75, S. 326; Mulisch 75, S. 198 
Vgl. (seltener) prät. Gerund auch zur Angabe der Vorzeitigkeit: 8 citavstichi, ja vsegda cuvstrujusebja legre

(Paffen II,S.363)

8.1.2. Skr. Ger + N + V

Im Russischen ist die Isolierung des untergeordnet prädikativen Gerund in Anfangsstellung obligat: 9 dies ist begrüdet durch die struktur der asymmetrischen Korrelation, insbesondere durch die Ersetzbarkeit in dieser Position durch sekundärprädikatives akt. Partizip.Im Skr. existiert diese Korrelation nicht, es steht nur das Gerund zur Verfügung, das der Isolierung zur Signalisierung seiner Funktion nicht bedarf.

Demgemäß halten sich in unserem Material bei Anfangsstellung des skr. Gerund isolierte und nichtisolierte Belege ungefähr die Waage. Isolierung erscheint hier eher als ein zusätzliches und fakultatives Grenzsignal.

Die Tendenz zur Isolierung erscheint dabei deutlicher ausgeprägt bei Erweiterung der Gerundialkonstruktion; in Analogie zu den oben angeführten russischen Belegen für Ger+I+N+V werden die folgenden Beispiele interpretiert.

Präs. Gerund:

U nekakome selu pođu zene u planinu da kaž divljega broća, i tako vrljajuci po planini jedna od njih zade... (Musić 35.5.146)

Dobro poznajući komesera, Uča je ovaj njegov postupak tumatio kao lukavstro (Tezak-Babić, S. 249)

Ne radeci plug te zardati = Ako ne bude radio, plug ce zardati (Tezak-Babić,S.249)

Prät. Gerund:

... I onde izgrlivsi se njom i izljubivsi otac i mati vrate se kukajuci (Musić 35,S.144,vgl.M..S.148) Cuvi ovo car mnogo se udivi (Musić 35, S.144)

8 Zu ipf. prät. Gerund vgl. ausführlich Paffen II, S. $363 f f$

$9 \mathrm{Vgl}$. als (nichtadverbialisiertes) Gegenbeispiel allenfalls: idja domoj ja povetretal Sonju (n. Morison 59,5.28 nicht korrekt) 
Izvršivsi svoju duznost, ti ćes dati primjer koleblivcima= Ako izvrsiś svoju dužnost...

(Tezak-Babić,S.250)

Von besonderem Interesse sind Belege, bei denen entweder die Isolierung nach dem subjekt steht und damit Gerund und subjekt zusammenfaßt oder, mit derselben wirkung, Erweiterungen des Serund nach dem Subjekt stehen, so daß das Subjekt "eingerahmt" wird durch die Gerundialkonstruktion. Im Russischen existiert diese Distributionsmöglichkeit nicht, wohl aber für aksl. $\mathrm{P}_{k}$. Vgl . :

Isavi on za dugo dode u jedan carski dvor (Musić 35,S.147) slusajuci ga ljenivac, stane mu zavideti govoreci

(Music 35, S. 146)

Derartige Belege sind in unserem Material selten. Unsere These lautet, daß bei Isolierung des Gerund (Ger+I+N+V) das Gerund als Thema gegenüber dem Restsatz fungiert wie auch im Russischen. Diese Funktion duirfte auch bei nichtisoliertem Gerund in vielen Fällen vorliegen. Möglich allerdings wäre hier, wie auch die oben angefuhrten Belege mit Zusammenfassung von Ger $+N$ zu einem (isolierten) syntagma nahelegen, thematische Zusammenfassung von Ger $+N$ gegenüber dem Prädikat als Rhema (s.u.). Im Russischen ist Isolierung obligat, im Skr. könnte,je nach aktualer Gliederung, Nichtisolierung gegenüber $\mathrm{N}$ obligat sein. $\mathrm{Zu}$ motivieren wäre diese vermutung aufgrund der angeführten Beispiele und der Situation im Aksl.

8.1.3. Aksl. $\mathrm{P}_{k}+\mathrm{N}+\mathrm{V}$

Im Aksl. tritt die merkmallose Kurzform des Partizips in dieser Distribution eindeutig peripherprädikativ auf ${ }^{10}$ (vgl. eindeutig attributiv: $\left.P_{1}+N+V\right)$. Der wesentliche Unterschied gegenuber dem russischen isolierten Gerund in Anfangsstellung liegt im Charakter der peripheren Prädikativität: Im Aksl. besteht eine untergeordnet prädikative Beziehung zwischen $P_{k}$ und subjekt, 11

$10 \mathrm{Vgl}$. Rủzicka, SS, S. $14 \mathrm{ff}$

11 Vgl.a.a.O. S. 20ff, S. 21: "Partizip und subjekt nehmen ... 
die nicht vermittelt ist über das satzschließende Prädikat wie im Falle der sekundären Prädikativität des russischen Gerund, sondern $P_{k}$ und subjekt zusammenfaBt als "isoliertes" peripherprädikatives Syntagma, das als solches dem Satz gegenübersteht und gleichzeitig angewiesen ist auf die satzschließende Hauptprädikation. Diesem Sachverhalt des peripherprädikativen Syntagmas und der merkmalhaften Nichtprädikativität der Langform des Partizips entspricht es, daB die Isolierung im Aksl. nicht die funktionale Relevanz hat wie im Russischen und, soweit uberhaupt erschließbar aus der Interpunktion, eher die Fügung $P_{k}+N$ zusammenfaBt statt $P_{k}$ von $N z u$ trennen, wie dies im Russischen der Fall ist für isolierte Partizipien und Gerundien in Anfangsstellung. Beide Möglichkeiten scheinen sich im Skr. abzuzeichnen, sowohl Ger $+I+N$... wie im Russischen als auch (selten) Ger+N+I... analog zum Aksl. Gemeinsam hat dabei das Skr. mit dem Aksl.. daß nicht $z$ u rechnen ist mit untergeordnet prädikativem Partizip (Langform). Während im Russischen die strukturelle Unterordnung des untergeordnet prädikativen Gerund (und Partizip) unter die satzschließende Prädikation abgeschlossen ist, reflektieren die Verhältnisse im Aksl. die alte Satzgeltung des Partizips mit der noch vorhandenen Möglichkeit zu annähernd parataktischer stellung ${ }^{12}$ gegenüber dem satzschlieBenden Prädikat. Hierauf verweisen neben der (nicht konsequenten) Zusammenfassung des Syntagmas $\mathrm{P}_{k}+\mathrm{N}$ durch die Interpunktio ${ }^{3}$ die Möglichkeit der Umrahmung des Subjekts durch Erweiterung des Partizips sowie die (genuin slavische) Möglichkeit des Gebrauchs einer koordinierenden Konjunktion vor dem Hauptverb. 14

11 ... im Satz eine stellung ein, die mit dem Terminus "isoliertes Syntagma" allgemein charakterisiert werden könnte. Wir haben gleichsam zwei Ebenen der Prädikation im Satz. Eine Analyse der "unmittelbaren Konstituenten" ("immediate constituents") wird deshalb der Gliederung nur gerecht, wenn Partizip und subjekt trotz ihrer prädikativen Beziehung als "immediate constituent" der höchsten Gliederungsstufe, der dem Prädikat gegenübersteht, angesehen werden könnten.": vgl. a.a.o.. s. 23

$12 \mathrm{Vgl} \cdot \mathrm{a.a.O.,S.} 16 \mathrm{ff}, 109$

$13 \mathrm{Vgl}$.a.a.0., s. 24

$14 \mathrm{Vgl} . \mathrm{a.a.0..} \mathrm{S.} 102 \mathrm{ff}$ 
Angesichts dieser unterschiedlichen Verhältnisse ist es irrefürend, vorgeblich übereinzelsprachliche Satzglieder gleichermaßen zur Funktionsbestimmung in verschiedenen Sprachen heranzuziehen, also z.B. dem $\mathbf{P}_{\mathbf{k}}$ in Anfangsstellung die Funktion eines (russ.) "obosoblennoe predikativnoe obstojatel'stvo" zuzuschreiben. Insbesondere in solchen fällen rächt sich, daß diese traditionellen Satzglieder heterogen sind und weder hinsichtlich ihrer formalen noch der semantischen Implikationen exakt definiert sind.

In unserer Magisterarbeit hatten wir vorgeschlagen, die aksl. peripherprädikativen Strukturen mit $\mathrm{P}_{k}+\mathrm{N}+\mathrm{V}$ dadurch von dem russ. Typ Ger+I+N+V zu unterscheiden, daB im Aksl. bei der Ableitung das identische subjekt im Rahmen des isolierten Syntagmas $P_{k}+N$ erhalten und für $V$ getilgt ist, während im Russischen die Tilgung des identischen Subjekts im Rahmen der Ableitung der Gerundialstruktur erfolgt. 15

Rủzicka nennt für das Aksl bei der Distribution $P_{k}+N+V$ als weit überwiegend Partizip von Verben der sinnlichen und intellektuellen wahrnehmung und der ortsveränderung im weitesten sinne. 16 Soweit in unserem Material zu erkennen, ist eine solche Tendenz im Russischen nicht zu beobachten; auch im Altkirchenslavischen überwiegen Präteritalformen gegenüber Präsenspartizipien. 17 Vgl. z.B.:

si rekb is. izide ss uceniky svoimi J 18,1 (SS,S.19)

i videvr is vérg ichn. rece oslablenuemu Mt 9,2 (SS,S.24) prizuravo is uceniky suoje gla imb Mr 8,1 (SS,S.25)

Mit Konjunktion (i) zwischen Partizip und Prädikat: vo ono vréme. slyšav irodh tetraarchb. sluchr chvr. i rete otrokomn svoimn Mt 14,1/2 (SS,S.104) Aus $\mathrm{MS}^{18} \mathrm{vgl}$. 2.B.:

iso davnna zlndajuste tebe ... caru ... Michailju Asénju ... azs

$15 \mathrm{Vgl}$. M., S. $41 \mathrm{ff}$

16 Vgl. Rủzićka, SS, S. 14ff; ähnlich für das Skr. vgl. Musić 35. S. $135 \mathrm{ff}, 142 \mathrm{ff}$

$17 \mathrm{Vgl}$. Rủzicka, Ss, S. $63 \mathrm{ff}$

$18 \mathrm{Vgl}$. M.. S. $36 \mathrm{ff}$ 
Marbsli Geörbgi ... i vise sudie i vekunici i öpokina vosa ... grada nasega Djubrovonika ... l jubov carostra ti imeti zibdajugte vo boga... klonemb se, öbestamo se

(MS, S. 35)

Vgl. mit Koordinierung mit finitem Verbum:

i sije prilo(zi)vori i zapisavosi kraljevostromi Svetoi Gore, i molju, jegoze bogb izvoli kraljevostrovati..., molju se jemou nepotvorenou byti

(MS, S.116)

Indiz für den engen Zusammenhang des peripherprädikativen Syntagmas $P_{k}+N$ im Aksl. ist die Tatsache, daß neben $P_{k}+N+V$ zwar möglich ist $N+P_{k}+V$, kaum aber Inversion von subjekt und Prädikat. 19 dies im Unterschied zum Russischen (vgl. Ger+I+V+N).

Z.B.:

trgda ostavo narody ide vo domn is.

Mt 13,36 (SS, S. 29) 20

Dies ist, soweit ersichtlich, das einzige Beispiel für $P_{k}+V+N$, das Ruzicḱa anführt.

Auch in MS finden sich kaum Belege; allerdings ist eine definitive Aussage hier schwierig angesichts des in den Urkunden oft fragmentarisch und vielfach verschachtelten Satzbaus, der die Satzgrenzen gelegentlich kaum oder gar nicht erkennen läßt. 21 Vgl. z.B.:

slyseste oubo sii velikaja obetovanija.. sego radi tostimuse i myi ... slysati (MS, S. 73)

Vgl. hierzu mit finitverbaler Prädikation:

slysachomb bo sii velikaa obetovanija ... sego radi tostimb se i my i ...

(MS, S. 78)

Vgl. hierzu auch die Belege mit Wiederholung des identischen Subjekts ${ }^{22} ;$ z.B.:

da mi vlastele i ōpkina ... smislivbismotrbse... chotésmo

19 Vgl. Rưzicka, SS, S. 29, 157

20 a.a.0.. S. 29: "Das Subjekt ist hier vom Ubersetzer der Deutlichkeit halber nachgetragen. Es wirkt in dieser Stellung uberhaupt wie ein unorganischer Nachtrag."

$21 \mathrm{Zu}$ Beispielen mit $\mathrm{P}_{k}+1+\mathrm{V}+\mathrm{N}$, für die wir Interpretation ana$\log 2 u N+P_{k}+i+V$ vorschlagen, vgl. M.. S. $40 f f$

$22 \mathrm{Vgl}$. M., S. $41 \mathrm{ff}$ 
knezs, vlastele $i$ vsa öpkina... (MS , S . 237-238)

8.1.4. Russ. $P+I+N+V$

In grundsätzlicher Analogie zu den oben angeführten Beispielen für Ger $+\mathrm{I}+\mathrm{N}+\mathrm{V}$ werden die folgenden Belege mit russ. Partizip in Anfangsstellung und Isolierung interpretiert. Dies ist die syntaktische Konsequenz der asymmetrischen Korrelation mit merkmallosem Partizip im Russischen (s.o.).

Vgl. zur Äquivalenz kongruierender und akongruenter strukturen z.B.:

Legko spravljajas' (spravljajušijsja) so vsemi zadanijami, na étot raz utenik ne mog resit' zadaci (AG 70,S.644,Rủz.73,S.457) vyrossi (vyrosij) vo sem'e istorikov, Karl Bajmakov s detstva uvlekalsja archeologiej (Adamec II 75,S.185,187) Kombination kongruierender und akongruenter strukturen: 23 Buduci ne v silach spravit'sja s soboj, pobelevsijot stracha i obidy, on skazal to, cego ne dolzen byl govorit'

(Adamec II 75, S.187)

I berezno prinjav ekspress, s velikoj spravivsijsja dal'ju, pod nim on (most) grjanul, kak orkestr, svoej ozvucennoju stal'ju (Gnevko 75.S.73)

I daze teper', voloka po luzam svoi bol'nye nogi v mokrych untach, golodnyj, terjajuscij soznanie ot boli i ustalosti, proklinaja luzi, vjazkij sneg $i$ rannjuju grjaz', on vse ze zadno vdychal chmel'noj vlaznyjaromat = teper',kogda on volocil..., on vse ze zadno vdychal ...

(Morison, S.58)

Kongruierende Form in Anfangsstelluna:

Mracnyj, postarevsij, on vvalilsjak Listratu

(Kŕižková 69,PO,S.29)

Sobrannyj, naprjazennyj, odnoj rukoj kasajuścijsja mogily, drugoj kolybeli, ja ošcušcal sebja mgnovennym i velikolepnym, vspus koj molnii, poglošcennyj mrakom (Rúzicka 66,S.50)

Budto prişedsij otkuda-to iz daleka, on teper' na vse smotrel

$23 \mathrm{Vgl}$. demgegenüber zum Ċech. Rủzicka 63,TYp., S. 845 
po-drugomu

Iskavij vo vae éti dva mesjaca chot'kakoj-nibud' tocki, na kotoruju mog by operet'sja...; Euvstrovavij, tto ełce novitok na izbrannoj doroge ...; s otcajanija resivijija ... na polnuju naglost', no ne smevsij resit'sja na eto pered Nastas'ej Filipovnoj..; pokljavijaja vaemi kljatvami bol'no naverstat'vse éto vposledstvii $i v$ to ze vremja rebjaceski mectaviji inogda pro sebja...; on dolzen teper' ispit' esce étu uzasnuju tašu... (RUzicka 66,S.49)

veera esce busevarsee, segodnja more uspokoilos'

(AG 70,5.644)

ozidavij bol'sogo soprotivlenija, Romanov poterjal ravnovesie i potascils ja

(Rudnev 63,S.162)

In der Literatur wird $2 . T$. die sekundärprädikative Funktion des russischen akt.Partizips in $\mathrm{P}+\mathrm{I}+\mathrm{N}+\mathrm{V}$ ubergangen oder in ihrer Spezifik durch eine an der morphologischen Form orientierte Terminologie verdunkelt.

$D a ß$ einschlägige Beispiele nicht sehr häufig sind, ist dabei kein Argument. Diese Funktion der Partizipialstrukturen wird deutlich nicht nur bei Kombination kongruierender und akongruenter Formen oder ihrer Ersetzung durch einander sowie durch die Unmöglichkeit der Relativsatzparaphrase, sondern auch durch die Möglichkeit der Inversion von Subjekt und Prädikat bei Anfangsstellung des isolierten Partizips.

Vgl. $2 u$ russ. $\mathrm{P}+\mathrm{I}+\mathrm{V}+\mathrm{N}$ :

Na poroge, izzjabsaja, stojala Sabel'nikova (Gnevko 75,5.75) Derevjannye i kirpicnye, krabenye i potemnevile, krytye tesom, zelezom i fiferom, mirno postaivajut tumanovskie doma

(Gnevko 75, S.70-71)

Sijajusciji veselyj, prinjalsja Il'ja vétot vecer za svoe obycnoe zanjatie (Kornilov 67.5.37)

Die Bedingungen der Wahl zwischen Partizip und Gerund bedürften im einzelnen noch der Untersuchung: wir vermuten, daß diese Bedingungen nicht mehr auf der Grundlage des Satzes, sondern der des Textes zu erörtern wären. 24

$24 \mathrm{Vgl}$. Mistrik 75, S. $645 \mathrm{ff}$ zur Kongruenz als riickverweisendem Mittel der Hypersyntax 
Die "Ersetzung" des Gerund durch Partizip in sekundärprädikativer Funktion findet insbesondere bei Anfangsstellung statt, wie sie hier skizziert wurde. Ruzicka ${ }^{25}$ sieht die Anfangsstellung als obligat, U.E. ist z.B. auch bei Zwischenstellung $(N+I+P+I+V)$ sekundärprädikative Funktion nicht ganz auszuschließen (s.u.). Nicht findet sich bisher, und in diesem Sinne wäre Ruzicka 66 , S. 49 beizupflichten, sekundärprädikatives akt. Partizip in $\mathrm{N}+\mathrm{V}+\mathrm{I}+\mathrm{P}$; vgl. allenfalls mit Gerund und/oder Partizip pass.: Volny b'jutsja i ropscut, zagrjazennye raznym chlamom

(Sint.r.j. 74,S.138)

Ja echal v gorod oren' medlenno, nikogo ne vstrecaja, nikem ne obognanny

(Rưzicka 73,s.470)

Neben Bedingungen der Wortstellung scheinen für die wahl des (merkmallosen) Partizips u.a. auch Tempus und Aspekt wesentlich zu sein. "Die Regularitäten und Bedingungen dieser strukturen sind jedoch noch kaum erforscht." 26

Rúzicka vermutet Ersetzung insbesondere des präteritalen ipf. Gerund durch Partizip.

In dem von uns durchgesehenen Material wird fuir Gerundialstrukturen in isolierter Anfangsstellung z.T. Koordinierung als Paraphrase/Ubersetzung angefüht, nicht aber für Partizip. Dem mag der Hinweis bei Rủzicka 73 entsprechen, wonach die kongruierende Form in noch höherem Grad als die akongruente kiondensatcharakter hat und das russische Gerund in seiner Ableitungsgeschichte zuruickgeht auf finitverbales Prädikat, das Partizip aber auf Satz mit Prädikatsnomen in generisch-klassifikatorischer Bedeutung.

Die Isolierung ist bei $\mathrm{P}+\mathrm{I}+\mathrm{N}+\mathrm{V}$ maximal distinktiv. $\mathrm{P}+\mathrm{N}$ ist eindeutig nichtprädikativ (attributiv). Bei $P+N$ uberwiegt in unserem Material Präsenspartizip deutlich, bei $P+I+N+V$ präteritales Partizip; in $\mathrm{P}+\mathrm{N}$ ist das Partizip innerhalb des syntagmas hinreichend beschreibbar und im Satz permutierbar, das isolierte

25 Rožička 65, Verbalphr., S. 352

26 ders. 66, S. 50; vgl, auch zu den noch ungeklärten Bedingungen der Wahl ders. 73, S. $466 \mathrm{ff}$; ders. 59. S. 545 
Partizip in Anfangsstellung muB hinsichtlich seiner formalen Distribution und seiner semantischen Beziehungen im Hinblick auf das Satzganze beschrieben werden. Bei $P+N$ wäre Ersetzung durch Gerund ungrammatisch.

Vgl.:

podarivij mne knigu drug uechal

podarivsij mne knigu, drug uechal

podariv me knigu, drug uechal

(Adamec II 75, S.28)

Fundiert ist die merkmalhafte und typlogisch charakteristische Signifikanz der Isolierung in russ. $\mathrm{P}+\mathrm{I}+\mathrm{N}+\mathrm{V}$ durch die nach Umwertung der Korrelation stark ausgebaute Möglichkeit der untergeordneten Prädikativität für die Partizipien mit Isolierung nach $N(N+I+P)$, während im Poln. und Cech. $N+P$ anders als im Russischen häufig ist. Die im Russischen in $P+I+N+V$ häufig gebrauchten prät. akt. Partizipien fehlen im Cech. und Poln. 27 Auf die Bevorzugung hypotaktischer Explizitheit statt untergeordnet prädikativer isolierter Partizipialkonstruktionen auBerhalb des Russischen wurde bereits in Zusammenhang mit $N+I+P$ verwiesen. 28

Vgl. zur Ersetzung von russ. $P / G e r+I+N+V$ durch Cech. adverbialen Nebensatz z.B.:

Vyrosij v niscete, Pavel vrazdebno otnosilsjak tem, kto byl $\checkmark$ ego ponimanii bogatym = ( Cech.): Pavel, protoze vyrost v bídé, mêl neprátelsky poměr k tém, kdo byli v jeho predstavách bohat 1 (Sint.r.j. 74,S.135)

Utomiv夕is', on poterjal interes ko vsemu, cto proischodilokrugom = (cech.): Protoze byl unaven, ztratil zájemo váe, co se dělo kolem něho

(sint.r.j. 74,S.135)

Vyslusav menja vnimatel'no, vy legko pojmetemenja = (cech.): Kdyz mé pozornè vyslechnete, snadno mi porozumite

(Sint.r.j. 74,S.138)

27 Zum Cech. vgl. Rứzidka 63, Typ., S. 837ff, 841, 853, ders.62, Korr.. S. 684: zum Fehlen der Formen auf-vii im Poln. vgl. Weiss 77, S. 69ff; allgemein zur Opposition (statt Korrelation) zwischen poln. Partizip akt. und Gerund vgl. Weiss 77, S. $227,352 \mathrm{ff}, 377 \mathrm{ff}$

$28 \mathrm{Vgl}$. z.B. Sint. r. J. 74, S. $138 \mathrm{ff}$ 
Vgl. auch:

Vstrezennye druz'jami, my bystro dobralis' do noclega= (Cech.): Že nám p̌ratelé příšli naproti, dostali isme se rychle na nocleh (Sint.r.j. 74.S.139)

Nizko opustiv golovu, mat' vylla iz komaty = (slk.):S nízko zvesenou hlavou vyšla matkaz izby (Svetlik 70,5.268)

8.1.5. Signifikanz der Anfangsstellung

Das bisher vorgestellte Material für Anfangsstellung des untergeordnet prädikativen Partizip und Gerund zeigt, daß weder Kriterien der zeitenfolge noch $\mathrm{zu}$ vermutende semantische Varianten Aussicht bieten auf eine durchgängige Klassifizierung der Funktionstypen. Es ist daher nach der Signifikanz der Anfangsstellung selbst zu fragen. Ihre Relevanz ist evident für Adverbialstrukturen mit Bezug auf das Satzganze (s.0.). 29

Carefully, Sam sliced the salami (Bartsch,V. 72,5.19)

v zooparke on risoval iivotnych: On risoval zivotnych v zooparke (s.o.)

A doma on chocet rabotat' $v$ sadu (Vitek 67,S.81)

= Buduti doma, on chotet rabotat' v sadu

vodvore igrajut deti: Deti igrajut vo dvore

(Adamec in Danes 74 (ed), S.193)

Nur bei Anfangsstellung sind die folgenden Paraphrasen möglich, die Bezug auf das Satzganze explizieren:

Vo dvore prisutstrujut igrajuscie deti

Vo dvore proischodit igra detej

Dvor javlajetsja mestom, gde igrajut deti

v Sant-Jago my zili v dome Pablo (Kŕizková 68,S.42) 30

$29 \mathrm{Vgl}$. Vitek 67, S. 70, 81ff, 98: "In addition to this we wish to refer to our discussion of adverbs of prepositional phrases with spatial or temporal value. It was pointed out that the clause initial position is a marked position for them clearly indicating their function as predication modifiers."

30 Vgl. Kłižková 68, S. 42: "Esli ze odno Adv otnositsja k jad$r u$, a drugoe $k$ ischodnej casti, to stanovitsja jasnym, tto neposredstvennoj sintaktsiceskoj svjazi mezdu nimi na samom dele net..." 
In diesem Zusammenhang ist auch von Interesse, daß die von Steinitz angeführten Sonderfälle mit obligatem Advb und fakultativem $A d v^{31}$ sich zunächst einmal durch die Wortstellung unterscheiden: das sonst fakultative Advb,jetzt bei verben. die eine Ergänzung fordern, steht am Satzende, das sonst infolge Valenzeigenschaften des übergeordneten Verbs obligate Adv steht jetzt am Satzanfang, während sonst Endstellung üblich ist.

Dem Unterschied in der Wortstellung entspricht der der aktualen Gliederung hinsichtlich Thematisierung/Rhematisierung. 32 Vgl.:

obligates Advb: Der Unfall mit der Rehgruppe geschah aus Unachts amkeit

fakultatives Adub: Auf dem Bahnhof fragten wir nach der Abfahrozeit des Zuges= Als wir auf dem Bahnhof waren, fragten wir nach der...

In der Konsequenz unserer Uberlegungen zur strukturierung des Prädikatskomplexes und der Signifikanz der Anfangsstellung sehen wir die Partizipial- und Gerundialstrukturen in P/Gert $\mathrm{I}+\mathrm{N}+\mathrm{V}$ als gegenüber dem gesamten Restsatz thematisiert an. Diese Strukturen stehen auBerhalb der Negation des satzschlieBenden Prädikats 33 und beziehen sich als Präsupposition auf das Satzganze. Diese Tatsache des durch die aktuale Gliederung

$31 \mathrm{Vgl}$. Steinitz 69, S. $26 \mathrm{ff}, 30 \mathrm{ff}, 38 \mathrm{ff}$

$32 \mathrm{Vgl}$. kritisch zu Steinitz hins. Adv Bartsch 72, S. $328 f \mathrm{f}$; zu obligaten Adub bei Steinitz vgl. Bartsch 72, S. 332: "Steinitz bemerkt nicht, daß ihre Behauptung, die erwähnten verben müßten eine obligatorische Ergänzung haben, nur in den Fällen richtig ist, in denen das Subjekt eine definite Deskription ist, bei deren Verwendung ja die Existenz des im Satzsubjekt Angegebenen bereits vorausgesetzt ist. Dagegen sind, -was Steinitz entgeht- die obigen ungrammatischen Sätze mit einem unbestimmten Artikel im Satzsubjekt durchaus grammatisch und sinnvoll: Ein Feuer (im Keller) war entstanden / Ein Unfall mit der Rehgruppe geschah."

33 S.O.: vgl. Sgall u.a. 73, S. 245: "As we have shown in 54.1. , the primary function of linguistic negation (as distinct from logical negation of an assertion) is in negating the relationship between the topic and the focus. The topic is thus outside the scope of negation, and so it is presupposed." 
konstituierten Satzbezuges liegt auch letztlich zugrunde, wenn nach im einzelnen wechselnden Kriterien, insbesondere mit Hinweis auf die lexikalische Semantik der beteiligten Komponenten und unterstellte Verhältnisse des außersprachlich Bezeichneten von zwei voneinander verschiedenen Handlungen, die zueinander in Beziehung gesetzt sind, gesprochen wird. Soweit wir diese Ausdrucksweise für unsere Zwecke übernehmen, ist sie nicht außersprachlich begründet, sondern durch den durch die kommunikative Intention des Sprechers intendierten Satzbezug. Für russische $\mathrm{P} / \mathrm{Ger}+\mathrm{I}+\mathrm{N}+\mathrm{V}$ nehmen wir folgende Gliederung an (unter der Voraussetzung einer "normalen" Satzintonation):

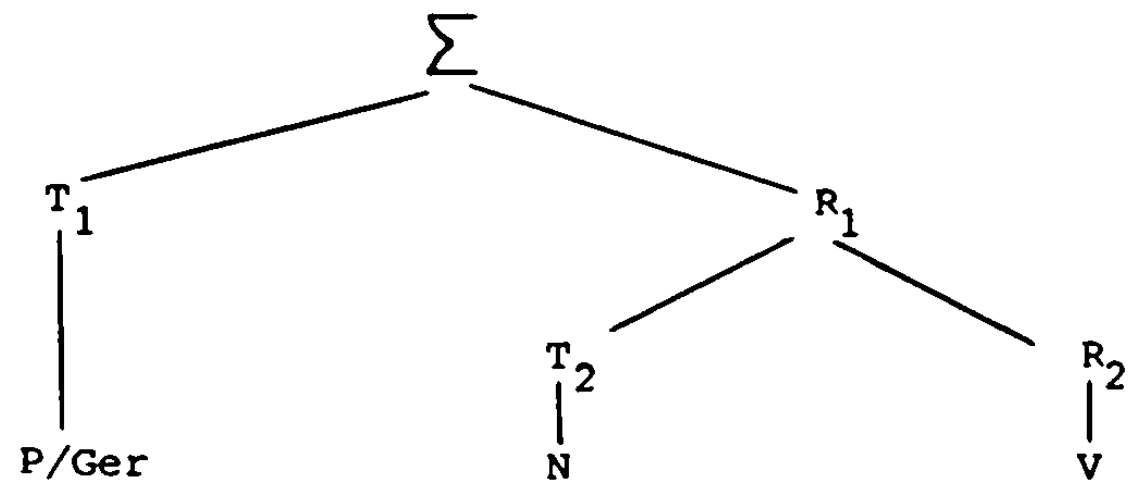

Analoge Interpretation wird angenommen fur die Mehrzahl der skr. Gerundien in Anfangsstellung. Nicht ganz ausgeschlossen scheint aber auch für Ger $+N+(I+) V$ :

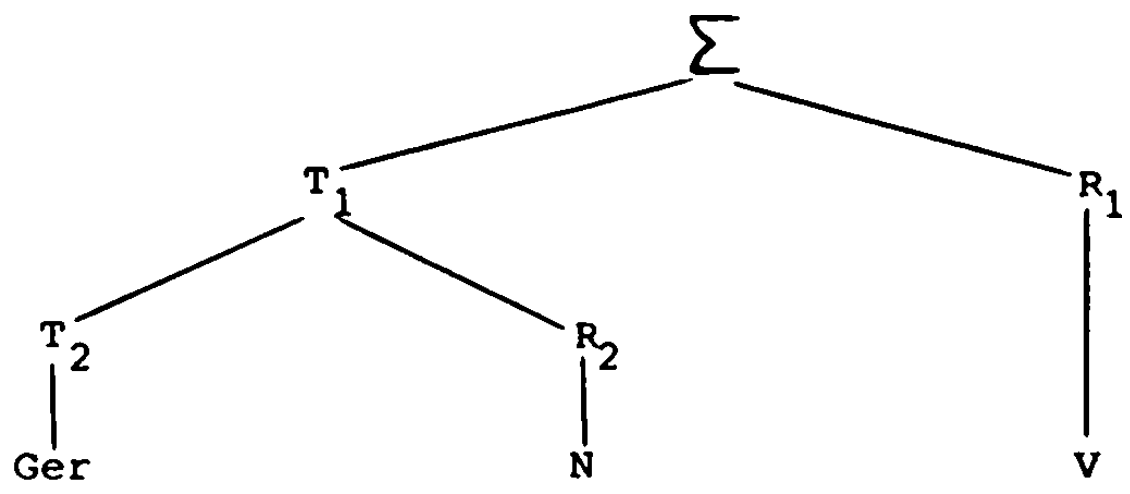

Zur Form der Schemata s.o. S. 158 
oder, Je nach Intonation, eventuell:

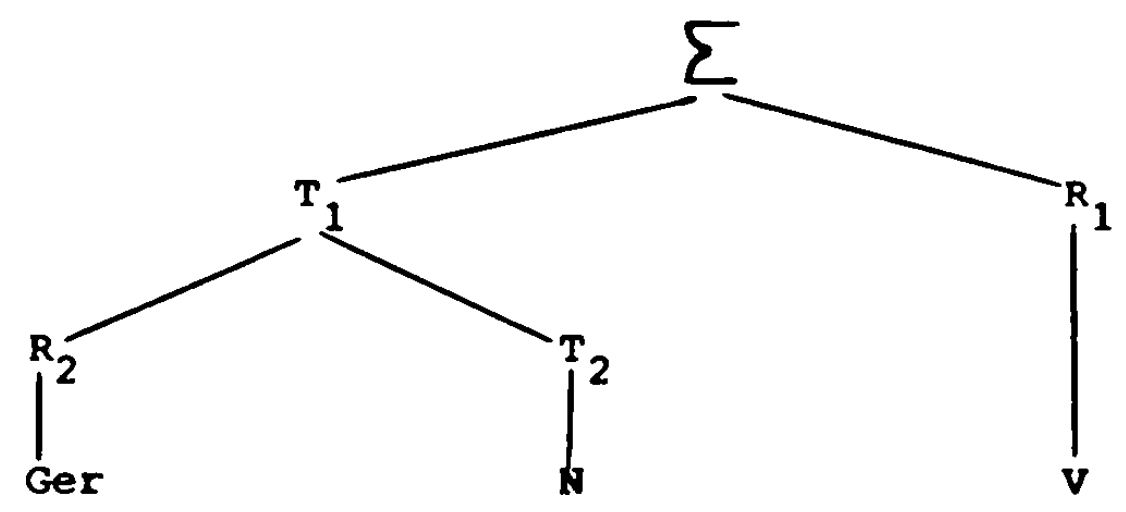

Dem spezifischen Charakter des aksl peripherprädikativen "isolierten Syntagmas" (vgl. Rủzicka, SS, S. 21ff) soll versuchsweise folgendes Schema entsprechen:

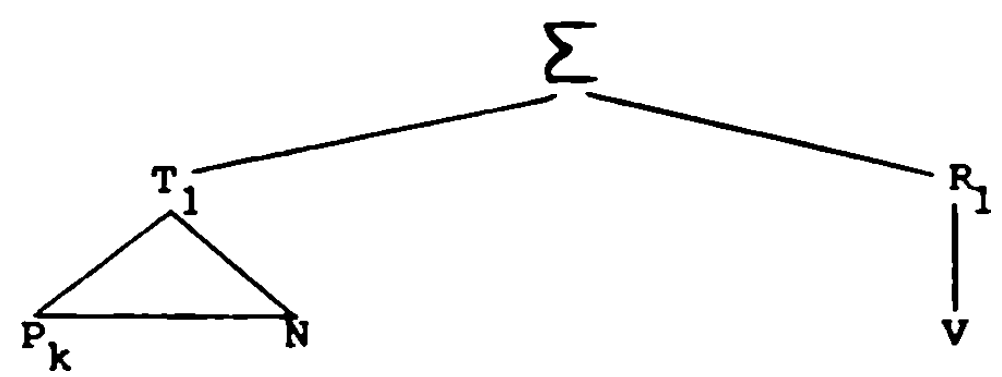

Die umfangreiche Diskussion zum "Satzdeterminanten" soll hier nicht erörtert werden. 34 Uns interessiert diese Möglichkeit der Funktion für Partizipial- und Gerundialstrukturen. Dabei ist grundsätzlich festzuhalten, daß diese syntaktisch fakultativ sind; sie sind nicht durch Fügungseigenschaften ubergeordneter Konstituenten, auch nicht im sinne einer "schwachen Rektion", $\mathrm{zu}$ beschreiben.

Die Relevanz der aktualen Gliederung für "Satzdeterminanten" in Anfangsstellung und die textuelle stellung des Determinan-

$34 \mathrm{Vgl}$. z.B. AG 70, S. $624 \mathrm{zu}$ Formen des Determinanten: vgl. a.a.O.: "Takoj samostojatel'nyj rasprostranitel' otnosjas sja ko vsemu predlozeniju v celom, nazovem determinantom" : Gabka 76, S. 204; Panzer 75, S. 170; Vitek 67, S. $129 \mathrm{zu}$ Brondal ("Situativ"); Svedova 73, S. 463; dies. 74,Gr.op.. S. 147ff: zur Kritik am bis anhin heterogenen Begriff des Determinanten, auch zu Švedova, vgl. z.B. Šmelev 76, S. $65 \mathrm{ff}$ 
ten, insbesondere seine Funktion der Rückverweisung auf den voraufgehenden Kontext, ist grundsätzlich bekannt, 35 nur eben, daß z.B. Pala, Uhlírová oder Adamec 66 die Funktion des Satzdeterminanten syntaktisch voraussetzen und ihr nachträglich Stellungen in der aktualen Gliederung zuordnen. In unserem Fall ist nur der umgekehrte weg gangbar.

Vgl. als Beispiel für die Rückverweisung auf den voraufgehenden Kontext:

Perecitav éti stroki, ja ulovil v nicb kakuju-to zatrudnennost' dychanija... (F, S.25)

Vgl. auch den ungewöhnlichen "relativen Satzanschluß" mit nichtisoliertem Gerund in:

vdrug vysla na luzajku kosulja, pocujav kotoruju moj Arto srazu neistovo rvanul povodok (Paffen II, S.377)

Die Anfangsstellung der Partizipial- und Gerundialstrukturen ist motiviert durch die aktuale Gliederung; die kondensierte Struktur steht als Thema dem Restsatz, der seinerseits wiederum kommunikativ gegliedert ist, gegenüber. Die ausgeprägte untergeordnete Prädikativität besteht zwischen diesem Thema und dem Satz als ganzem.

Diesen Sachverhalt beschreibt Weiss als Angabe der "Kulisse". des "Hintergrundes" mit der Modalität "supponiert wahr" 36 im Unterschied zur eigentlichen Assertion des Satzes.

Vgl. z.B. poln.:

Psioczac bez przerwy na dyrektora, ten pracownik zatruwa atmos fere w całym zakładzie (Weiss $77, \mathrm{~S} .301$ )

Nie czujac sie nadzwyczainie, Anna wrócita wcześniej do domu= Poniewaz nie czuza sig...

(Weiss $77, \mathrm{~s} .316$ )

Vgl. zur Nichtbetroffenheit durch Negation oder nichtassertive Modalität des Prädikats sowie zur Negation der kondensierten Struktur unabhängig vom Prädikat z.B.:

Russ .:

Cto slyşu? Edva nacinav rabotat', ty uze ustal? Ty bolen?

$35 \mathrm{Vgl}$. u.a. Buttke 69, S. 42ff, Grepl 75, S. 633, Sgall u.a. 73, S. 80

36 Weiss 77, S. $300 \mathrm{ff}$ 
Dovol'no togo, Rto, opirajas' na nich i cerpaja vich silu, ja ne otstupljus' ot svoego natinanija (F,S.188)

Ne ponimavi vse eto, starik ne znal, cto delat' = Weil der Alte das alles nicht begriff, wubte er nicht, was er tun sollte

(Mulisch 75, S.204)

Buduci v zooparke, on ne risoval zivotnych

Ne ogladyvajas', on medlenno sel po ulice (Isatenko 75,S.332)

Rodivsajaja za granicej, moja mat' ne govorit po-anglijski

Skr.:

Probudivi se oko ponoći, dijete nije plakalo

Aks 1.:

podobaase ti ubo. vidati surebro moe troḱnikomb. i prisodbazb. vorels ubo bimb svoe sb lichvojo

"Der irreale Modus des Konditionals erstreckt sich nicht auf das Partizip: ... Für die Gliederungsverhältnisse ist das Beispiel wieder charakteristisch: wo immer ein subjekt zwischen Partizip und Hauptverb steht, tendiert es zum Partizip und bildet mit ihm ein Syntagma."

(Rủzickka, SS, S.81)

i ako kto pribegne... u gradb nast, da u nast stoe nikoegore

21 a da ne ime ciniti (MS,S.48)

poiduke i priduke da ne tinu pristaniste u ... (MS,S.31)

(weitere Beispiele in M., S. 47ff)

Hinsichtlich möglicher Paraphrasen ergibt sich in dem für Anfangsstellung des Partizip/Gerund erörterten Material (neben der Nichtbetroffenheit durch die Negation des satzschließenden Prädikats) im ganzen folgendes Bild:

Relativsatz ist in jedem Fall ausgeschlossen.

Möglich ist Paraphrase durch Präposition + deverbatives Substantiv und adverbialer Nebensatz, wobei über dessen Varianten wie temporal, kausal usw. allenfalls statistische, nicht aber strukturelle Aussagen gemacht werden können.

Möglich ist grundsätzlich auch Koordination der zu einem finiten Prädikat umgewandelten kondensierten struktur mit dem satzschließenden Prädikat, allerdings mit der wesentlichen Einschränkung, daß bei unterstellter konditionaler Beziehung Um- 
wandlung $z u$ Koordination nicht möglich ist ${ }^{37}$ wie wohl überhaupt bei unterschiedlicher Modalität für kondensierte struktur und Satz.

Vgl.:

Poobedav, ja prisel $k$ vam = Ja poobedal i (posle étogo) prisel $k$ vam (Brave 40,S.25)

Zajdja ze ko mne vtoroj raz, on prjamo sprosil = On zasel ko mne vtoroj raz i prjamo sprosil (Morison, S.41)

Okonciv lekciju, professor besedoval so studentami = Posle okoncanija lekcii professor besedoval ... = Posle togo, kak professor okoncil lekciju, on besedoval ...

(Adamec II 75, S.12)

Ne vyrvav iniciativu Koľ̌aka, my budem otbroßzeny za volgu= Esli my ne vyrvem iniciativu Rolčaka, to budem otbrokieny za vol gu

(Ananič 72, S.62)

Im Sinne der oben diskutierten Klassifizierung lassen sich u.E. sämtliche russ. Belege des Typs $P / G e r+I+N+V$ zusammenfassen hinsichtlich der funktionstypkonstituierenden Relation als Thema gegenuiber dem Satz.

Nun unterscheidet z.B. Kade 68 zwischen in der Konstituentenstruktur prädikatsfernerem sekundärem Prädikat" und dem Zentrum der Prädikation näherem "Advb" und nennt für die hier angeführte Distribution auch die Möglichkeit der Funktion als Advb. Eine überzeugende Begründung fehlt, ebenso, wenn z.B. in der AG 70 auch für den hier vorliegenden Distributionstyp ad hoc unterschieden wird zwischen Determinant und obstojatel'stvennocharakterizujuścee znatenie. Es handelt. sich, soweit der prädikatsnähere Funktionstyp angenommen wird, zumeist um Belege, für die eine modale oder instrumentale semantik unterstellt wird, und die mit einer vielfach wechselnden Terminologie als "Merkmal einer Handlung" umschrieben werden aufgrund durch das außersprachlich Bezeichnete begründeter Assoziationen. Insofern besteht die Kritik bei Weiss 74, weiss 77, S. $341 \mathrm{ff}$ an der Unterscheidung von Advb und Prädsec $z u$ Recht. Damit ist

$37 \mathrm{Vgl}$. Rứicka, SS, S. 69; Weiss 77, s. $322 \mathrm{ff}$ 
aber noch nicht gezeigt, daß eine strukturelle Differenzierung des Prädikatskomplexes uberhaupt unmotiviert ist, sondern nur, da $B$ bei der vorliegenden Distribution eine Differenzierung nicht zu begründen ist. Die Möglichkeit der von Kade angeführten unterschiedlichen Paraphrasen soll dabei nicht bestritten sein, sondern nur ihre Ausschließlichkeit, d.h. ihre Signifikanz. Vgl. z.B.

nach Kade "Advb":

Rassiraja ... svoe viijanie vmirovoj politike, socializmstre$\operatorname{mits} \mathbf{j a} \ldots$ (Kade 68,S.614)

Razoblacaja obman reakcionnoj propagandy ..., kommunisticeskie i rabocie partii vnosjat ogromyj vklad v borbu...

(Kade 68,S.614)

Raskryv suscnost' kapitalisticeskoj ékspluatacii, Marks nanes smertel'nyj udar utverzdenijam ... (Kade 68,S.615) andererseits nach Kade "sek.Präd.":

Preodolev ego (Dekarta) dualizm, Spinoza obosnoval monisticeskoe materialisticeskoe ponimanie prirody (Kade 68,5.605) sleduja ükazanijam Lenina, central'nyj komitet partii pridaet bol'soe znacenie rabote po ... (Kade 68,S.605) Pol'zujas' étim, kapitalist platit rabocemu zarabotnuju platu (Kade 68,S.610)

Nach den im voraufgehenden diskutierten Kriterien sind die Belege bei Isolierung und Anfangsstellung einheitlich, d.h., auch bei den in der Literatur als "modal", "Advb", "Merkmal einer Handlung" usw. aufgrund von Mutmaßungen uiber das außersprachlich Bezeichnete unterschiedenen Beispielen in dieser Position ist insbesondere Negation des Prädikats unabhängig von der isolierten Wendung möglich.

Z.B.:

Raskryv suß̨nost' kapitalisticeskoj èkspluatacii, Marks ne nanes smertel'nogo udara utverzdenijam ...

Uberlegungen, ob auf der Ebene des außersprachlich Bezeichneten im Einzelfall möglicherweise die Gerundialhandlung "enthalten" ist in der Prädikatshandlung, führen nicht weiter. Entscheidend ist die vom sprecher intendierte stellung auf der Ebene der 
sprachlichen Bedeutung. Beachtenswert scheint uns, daß derartige Probleme in der Literatur ausschließlich in Zusammenhang mit Gerundien auftreten, während für Partizipialstrukturen bei Anfangsstellung und Isolierung die Interpretation "zwei voneinander verschiedene Handlungen" allgemein als gesichert gilt, der der Bezug auf das Satzganze zugrundeliegt.

8.1.6. Russ. Ger $+\mathrm{I}+\mathrm{V}+\mathrm{N}$

Anders als im Aksl. und in MS ist im Russischen der Stellungstyp Ger $+\mathrm{I}+\mathrm{V}+\mathrm{N}$, d.h. Anfangsstellung des untergeordnet prädikativen Gerund und Inversion von Subjekt und Prädikat durchaus möglich, allerdings kompliziert sich hier das Bild hinsichtlich der möglichen semantischen Beziehungen.

In Analogie $z u$ den oben angeführten russ. Belegen für Ger+I+N+V können U.E. die folgenden Beispiele mit Inversion gewertet werden:

Euja blizkuju zimu,vstrevoźnno karkali grjaci v verchuśkach ogolennych topolej (Rudnev 63,S.178)

Pochoroniv Aksin'ju, troe sutok bescel'no skitalsja on po stepi (Rudnev 63.S.177)

Dozd' po pseničnom polju pronesja - potjažlev, udlinilis' kolos'ja i poklonilis' (Gnevko 75, S.86)

Derartige Beispiele treten in unserem Material erheblich seltener auf als Ger+I+N+V; noch seltener, aber immerhin möglich, ist $\mathrm{P}+\mathrm{I}+\mathrm{V}+\mathrm{N}$.

In dem für deutsche nichtkongruierende Partizipialkonstruktionen angefürten Material,z.B. bei Bungarten 76, tritt demgegenuber ausschließlich $\mathrm{P}+\mathrm{I}+\mathrm{V}+\mathrm{N}$ auf; die Inversion ist hier obligat:

Gelangweilt dozierend, redeten sie auf ihn ein

(Bungarten 76,S.211)

Ohne Isolierung:

Stillschweigend nahm sie an der Prüfung teil

(Bartsch 72,S.142) 
Grundsätzlich ist bei den hier aufgeführten russischen Belegen Negation des Gerund und des Prädikats unabhängig voneinander möglich analog $z u$ den Belegen ohne Inversion. Umwandlung $z u$ einer finitverbalen Prädikation und Koordinierung wäre allenfalls bei Aufhebung der Inversion denkbar; mit und ohne Aufhebung der Inversion ist möglich Paraphrase durch adverbialen Nebensatz, dabei ergibt sich hinsichtlich der semantischen Varianten im ganzen ein entsprechendes Bild zu den Belegen ohne Inversion (vgl. Ubersetzung mit nachdem, weil usw.),d.h. "zwei voneinander verschiedene Handlungen" auf der Grundlage des Satzbezuges. Analog zu den oben angefürten Belegen sehen wir hier das Gerund als Thema gegenüber dem Restsatz.

Schematisch:

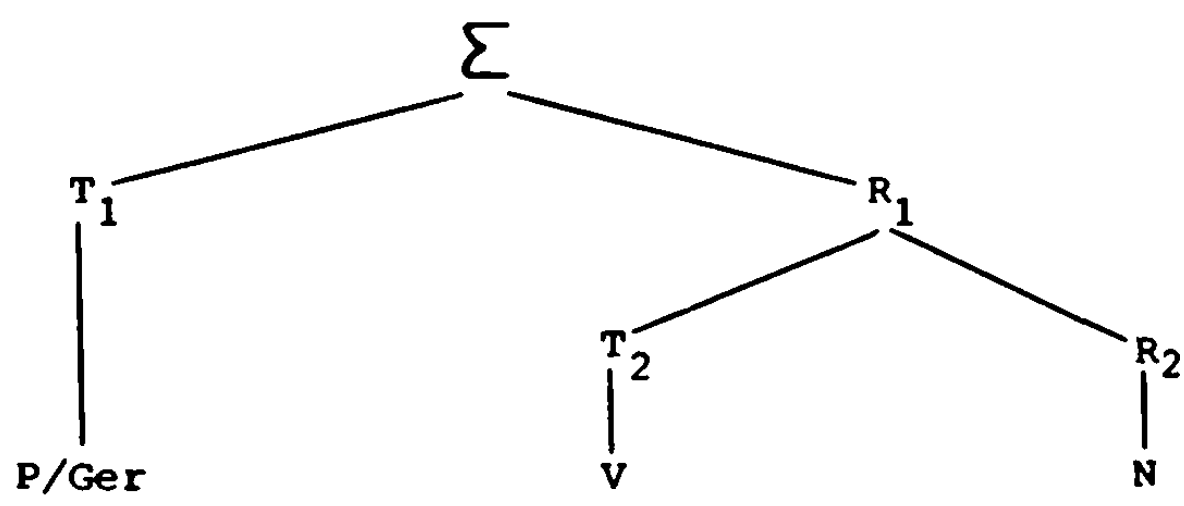

Anders als bei Ger $+\mathrm{I}+\mathrm{N}+\mathrm{V}$ ist aber die oben angegebene Interpretation nicht die einzig mögliche bei der Distribution Ger+I+V+N. Vgl. 2.B.:

Gde-to, budto zalujas', zavyval parovoz (Sint.r.j.74,5.137) Polusmejas', polu v vostorge otvetilon (Morison,s.31)

Po koridoru, zvonko otstukivaja kablukami, idet vysokaja devuska (Adamec II 75, S.171)

Cerezdvernuju scel', lozas' kosoj poloskoj na pol, probivalos' solnce

(Gnevko 75.S.85)

Zaloziv ruki za spinu, posvistyvaja idet Artjuska Pistolet. Mit hinter dem Rücken verschränkten Armen kommt A.P. vor sich hin pfeifend daher

(Paffen III,S.209)

Das zweite Gerund ohne Isolierung!

Eindeutige formale Kriterien sind nicht auszumachen für diese 
Gruppe: jedoch iberwiegen deutlich ipf. Aspekt des Gerund sowie Präpositionalkonstruktion (seltener Gerundialstruktur) vor dem Gerund, das hier in Frage steht. Die Untersuchung der Beziehungen zwischen z.B. mehreren Partizipial-und Gerundialstrukturen in Anfangsstellung oder innerhalb einer syntagmatischen Kombination mit Präpositionalkonstruktion hat noch kaum begonnen. 38 Soweit vorläufig festzustellen, kann, muß aber nicht, koordinatives Verhältnis auftreten:

?: Zaloziv ruki zaspinu i posvistyvaja idet Artjuska Pistolet S neprijatnym Euvstvom, prevozmogajakakoj-to neob-jasnimyj uzas, dolzen jazdes'soobsit', Eto i vtoromu ego vyboru suzdena byla neudaca

$$
(F, S .192)
$$

?: S neprijatnym cuvstvom i prevozmogaja...

Unmöglichkeit der Koordinierung als Indiz für hierarchische Zuordnung:

Po ovražku-tupičku, gremja, žurja, perelivajas', beìal ručeek

(Gnevko 75.5.83)

Die Gerundien sind hier gleichrangig und könnten, von stilistischen Bedenken abgesehen, kordiniert werden, nicht aber die einleitende Präpositionalphrase:

Po ovrazku-tupicku i gremja, zurja...

Po lesu, osekaja chvoju, scepaja sosny, s gadjuc'im sipom zaryvajas'vzemlju, skakali i, tmokaja, rvalis' razryvnye puli

(Rudnev 63.S.178)

Po lesu i osekaja ...

Für derartige Gerundialkonstruktionen gilt nicht Bezug auf das Satzganze, sondern auf das prädikat. Sie sind nicht anwendbar auf negierte Sätze. Paraphrasierung ist hier schwierig bis unmöglich; Ubersetzung mit adverbialem Nebensatz wäre allenfalls mit indem denkbar: Koordinierung mit dem Prädikat ist nicht plausibel. Bezeichnenderweise werden auch in der Literatur für derartige Belege keine Paraphrasen, sondern nur allgemein Umschreibung als "modale Bestimmung", als Angabe eines der Prädikatshandlung "immanenten Merkmals" u.ä. angeführt und auf die

$38 \mathrm{Vgl}$. z.B. auch Rúzicka, SS, S. 112ff, 125; Weiss 77,s.290ff 
Schwierigkeit der Ersetzung verwiesen. 39

Ausgeschlossen scheinen hier die oben angeführten Paraphrasen bzw. Ubersetzungen mit während, nachdem, weil usw.. Am zwanglosesten ergibt sich auch im Dtsch. Konstruktion mit nicht

flektierendem Partizip.

Im Unterschied $z u$ den oben angeführten Belegen für Gerund als Thema gegenuiber dem Restsatz schlagen wir vor, hier das Gerund zusammen mit dem Prädikat als Thema (das seinerseits gegliedert ist) dem subjekt als Rhema gegenüberzustellen:

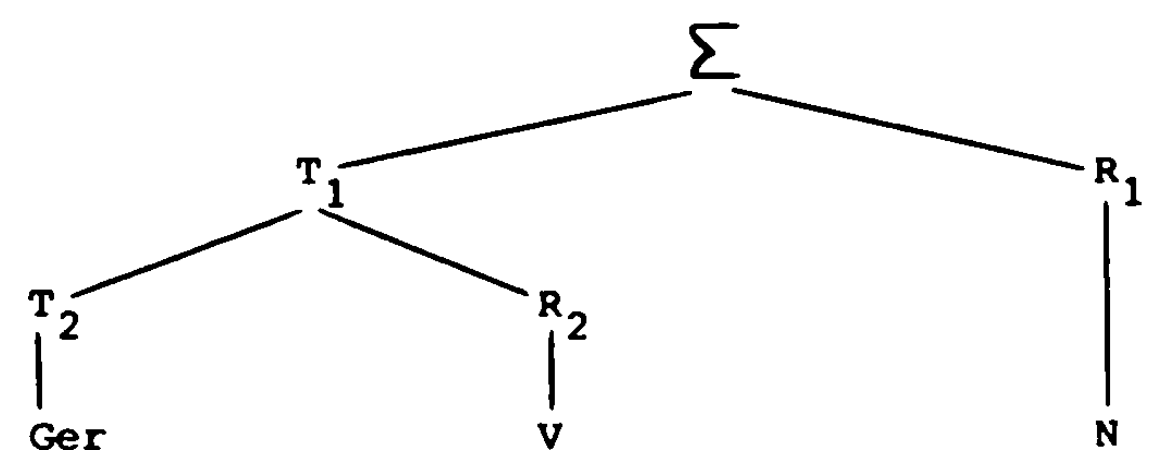

Hatten wir die oben angeführten Belege für Ger+I+V+N als Inversionen von $V$ und $N$ aufgefaßt, so liegt jetzt vor Inversion von $\mathbf{N}$ gegenüber dem gesamten Prädikatskomplex, bestehend aus Gerund und $V$. Das heiBt: Hatten wir die vorigen Beispiele in Analogie zu Ger+I+N+V beschrieben, wäre jetzt eher die Rückführung auf den Typ N+I+Ger+I+V denkbar: tritt vor das fragliche Gerund eine Präpositionalkonstruktion oder eine Gerundialkonstruktion, für die nicht Gleichordnung, sondern Bezug auf das Satzganze angenommen wird, ergibt sich folgende Erweiterung:

39 Vgl. z.B. Rudnev 63, S. 178; Mulisch 75, S. 208: Adamec II 75, S. 171 


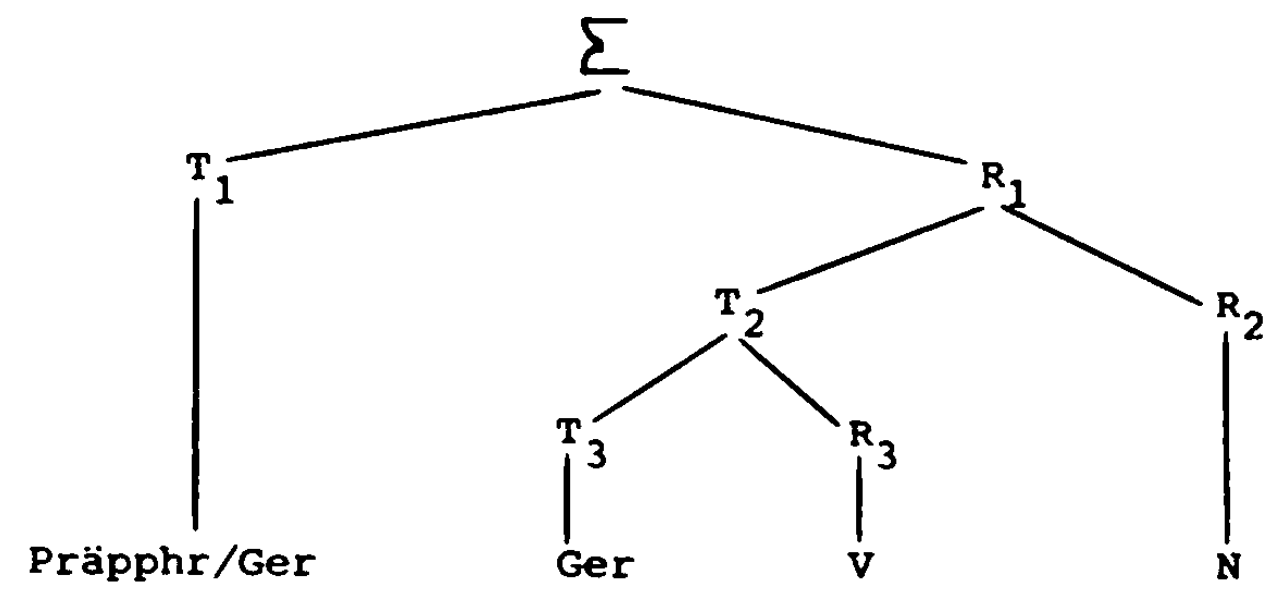

Wenn diese Uberlegungen plausibel sind, erscheint die Interpretation des folgenden Gerund als "kausal" zweifelhaft:

Pered muzikami, ne smeja prisest" v rjad s "chozjaevami", stojali dve zenskie figury $v$ temnch sarafanach

= ?: Pered muzikami stojali dve Zenskie figury ..., potomu Cto oni ne smeli sest' v rjad s "chozjaevami" (Svetlik,s.269)

Diese Paraphrase Svetliks gibt u.E. Auskunft über einen hinsichtlich des außersprachlich Bezeichneten möglichen zusammenhang, dessen Mitteilung so aber nicht intendiert ist; dann wäre eher Anfangs- oder insbesondere Endstellung des Gerund, und und zwar als Rhema gegenüber dem Restsatz, zu erwarten. Als deutsche Ubersetzung kommt eher in Frage ohne zu statt weil; vgl.:

Vor den Männern standen, ohne daß sie es gewagt hätten, sich zu setzen ... zwei weibliche Gestalten ...

demgegenüber:

?? Vor den Männern standen zwei weibliche Gestalten ..., weil sie es nicht wagten, sich zu setzen

8.2. Zwischenstellung

Hatten wir für Anfangsstellung der kondensierten Struktur und Nichtinversion von Subjekt und Prädikat ausschließlich Bezug auf das Satzganze infolge Thematisierung angenommen, so kompliziert sich bei zwischenstellung, d.h. bei $N+I+P / G e r+I+V$, das 
Bild. Es ist zu rechnen mit Bezug auf das Prädikat, auf das Subjekt und, nach dem Negationskriterium, mit Satzbezug. Ahnliche Probleme treten ja auch bei Adverbialbestimmungen auf; auf die Ambiguität der Zwischenstellung hinsichtlich Bezug auf das Satzganze : Bezug auf das Prädikat wurde bereits verwiesen für z.B.

Sam carefully sliced the salami

Eine andere Ambiguität liegt vor bei der folgenden lokalen Adverbialbestimmung:

Okno v stene zanimalo malo mesta

(Vitek,S.76)

Neben der naheliegenden Paraphrase zur Explikation der Zugehörigkeit zum subjektskomplex

okno, kotoroe v stene, zanimalo malo mesta schließt Vitek als "hypothetisch" zumindest nicht aus:

okno, buduci v stene, zanimalo... (Vitek, S.77)

in Analogie $2 u$ :

Student naverchu videl knigu (Vitek,S.76)

student, kotoryj byl naverchu, videl knigu

student, buduci naverchu, videl knigu

Dabei expliziert die Relativsatzparaphrase zuordnung zum Subjektskomplex, Paraphrase mit buduci... Bezug auf das Satzganze mit subjektsidentität für die der Adverbialstruktur zugrundeliegende Prädikation.

Eindeutig ist der Satzbezug bei Anfangsstellung:

Naverchu student videl knigu.

Dabei ist nochmals $2 \mathrm{u}$ betonen, daß für das vorliegende schriftlich fixierte Material die Intonation nicht zuverlässig rekonstruiert werden kann, die zumindest in einer Reihe von Fällen relevant wäre für die Disambiguierung.

Der grundsätzlichen Mehrdeutigkeit der Zwischenstellung entspricht es, wenn Weiss 77 den (oberflächensyntaktischen) Unterschied zwischen attributiven und sekundärprädikativen strukturen in dieser Distribution neutralisiert sieht, ${ }^{40}$ allerdings mit der wesentlichen Einschränkung, daß der morphologische Gegensatz zwi-

40 Weiss 77, S. $227 f f, 355,358 f f$ 
schen akt. Partizip und Gerund im Polnischen eindeutig die Zugehörigkeit zum subjekts- oder Prädikatskomplex signalisiert. Bungarten sieht für deutsche nichtkongruierende isolierte präsentische akt. Partizipialstrukturen in Zwischenstellung ausschließlich attributive Interpretation vor; auch wenn man sich in allen Fällen seiner Interpretation anschließt, wird hier u.E. genaugenommen nur gezeigt, daß im Deutschen für Relativsatz die stellung nach dem Bezugsnomen obligat ist und adverbiale Nebensätze mit Konjunktion in dieser Position ungramma$t i s c h$ sind, nicht aber, daß für die $z u$ interpretierende partzipialstruktur Zuordnung zum Prädikatskpmplex völlig ausgeschlossen ist. 41

Vgl. z.B. nach Bungarten, S. 155 attributiv:

Der alte Mann, stöhnend und ächzend, erklomm die Treppe.

8.2.1. Russ. $N+I+G e r+I+V$

Anders als bei Weiss ${ }^{42}$ für das Polnische wird für das Russische in seltenen ${ }^{43}$, aber doch immerhin mehrfach belegten Fällen als Ubersetzung/Paraphrase für Gerund in Zwischenstellung Relativsatz angefürt. Dies beeinträchtigt die sonst allgemein aufgrund der Wortart angenommene Nichtzugehörigkeit des Gerund zum Nominalkomplex und veranschaulicht die potentielle vieldeutigkeit der Zwischenstellung.

Vgl. z.B.:

Pravye socialisty, vypolnjaja volju amerikanskich imperialistov,

41 Filipović 77 , S. 40 legt ebenfalls attr. Interpretation für Zwischenstellung zugrunde, läßt jedoch immerhin für einige pf. pass. Partizipien die Möglichkeit der Zuordnung zum Prädikatskomplex offen: vgl. auch Krizková 6\%, Synt.ch., S. 120ff: Bungarten 76, S. 81ff, 150ff; Sviblova 62, S. 177ff

$42 \mathrm{Vgl}$. Weiss $77, \mathrm{~S} .189 \mathrm{ff}, 331$

$43 \mathrm{Vgl}$. 2.B. Mulisch 75, S. 209: "Die mitunter mögliche wiedergabe einer Adverbialpartizipialkonstruktion durch einen Relativsatz ist nicht typisch."; ähnlich Leitfaden 71, s. 149: "Diese Form kommt meist nur bei nachgestelltem Adverbialpartizip in Frage, ist aber nach Möglichkeit uberhaupt zu meiden" 
propagandirujut reakcionnye idei kosmopolitizma = Pravye socialisty, kotorye ...

(Leitfaden,S.149)

Mal'cik, spokojno stoja u vorot, smotrel na padajuscij sneg= Der Junge, der ruhig am Tor stand, betrachtete den fallenden Schnee

(Paffen II,S.375)

Perecen', ochvatyvaja lis'cast' vozmoznostej, dalek ot polnoty = Das Verzeichnis, das nur einen Teil der Möglichkeiten erfabt, ist weit entfernt von vollständigkeit

(Mulisch 75, S.209)

Slabyj veter, sevelja v list'jach jasenja, tichon'kodvigal vzad $i$ vpered, $i$ po temnoj dorozke, i po zeltoj spine sobaki, blednozolotye pjatna sueta = Der leichte wind, der im Laub der Esche spielte, ließ blabgoldene Lichtflecken langsam iber den dunklen Weg und den gelben Rücken des Hundes hin-und herschwanken. (Paffen II,S.375)

Tam perechodnaja sistema, osnovyvajas' na ruenoj primitivnoj technike, derzalas' vekami = Dorthielt sich das auf primitiver manueller Technik gegrüdete Ubergangssystem jahrhunderte1 ang. (Kade 68,S.614)

Revolucionnoe dvizenie v Rossii, ochvatyvaja bystro novye $i$ novye naselenija, sozdaet celyj rjad bezpartijnych organizacij (Kade 68.S.613)

Bei diesen Belegen liegt ipf. Aspekt des Gerund vor, d.h. das Argument "Gleichzeitigkeit trotz Stellung vor dem Prädikat". das Kade in Zusammenhang mit pf. Gerundien ${ }^{44}$ für die Zuordnung zum Subjektskomplex anführt, spielt hier keine Rolle.

vgl. mit pf. Gerund:

Gosudarstvennyj sekretar' Rask, vystupiv na presskonferencii, opravdyval nezelanie SśA prekraśçat' bombardirovki DRV ssylkami na to, cto drugaja storona jakoby ne zelaet dat otvetnych garantij. = ..Rask, kotoryjvystupil na ... = . Rask, vystupivij na...

(Kade 68,S.611-612)

Libo burzuazija, okrepnuv posle demokraticeskogo perevorota..., otnimet vse zavoevanija ot rabolich i krest'janskoj massy... (Kade 68,S.614)

44 Kade 68, S. 612 
Eine konsequente Erklärung derartiger Belege wird bis anhin nicht gegeben. Für alle in diesem Zusammenhang angeführten Beispiele gilt Nichtrestriktivität gegenüber N, Zwischenstellung und Gleichzeitigkeit der Gerundialhandlung oder möglicherweise bei den pf. Gerundien des Ergebnisses der Gerundialhandlung (vorzeitig-resultative Bedeutung) gegenüber der Prädikatshandlung; doch folgt weder aus der $\mathrm{Zwischenstellung} \mathrm{noch} \mathrm{aus}$ der Gleichzeitigkeit notwendig syntaktische zuordnung zum Subjektskomplex; nach Auskunft von Muttersprachlern ist für keines dieser Beispiele Relativsatz als Paraphrase obligat. In unserem Zusammenhang ist die hier vorgeschlagene Relativsatzparaphrase wesentlich als Indiz für die Ambiguität der Zwischenstellung. Probleme hinsichtlich der Definition der Korrelation mit dem merkmalhaft untergeordnet prädikativen Gerund ergeben sich dann nicht, wenn man strikt unterscheidet zwischen der untergeordneten Prädikativität selbst-die ist auch in diesen Fällen gegeben- und der Position der Einbettung.

U.E. ist in derartigen Fällen das Motiv für die Relativsatzparaphrase auf der Ebene der aktualen Gliederung zu suchen. Es handelt sich in keinem Fall um den Typ "eine Handlung mit ihrem Merkmal", sondern um Strukturen, die ihre Gultigkeit auch bei Negation des Prädikats behalten.

Vgl . :

Rask, vystupiv na presskonferencii, (ne) opravdyval ... In der aktualen Gliederung sind subjekt und Gerund zusammen dem Prädikat gegenüberzustellen. Derartige strukturen sind ein deutlicher Hinweis auf die Affinität von nichtrestriktiv-attributiven und sekundärprädikativen Strukturen und U.E. für die Relevanz der aktualen Gliederung bei ihrer syntaktischen Klassifizierung. Nicht jedoch ist damit ein Argument für das Gerund als "abstrakte Zwischenstufe der Ableitung" 45 auch für Partizipien im Rahmen der Nominalphrase geliefert.

Von den oben angefürten problematischen "Ausnahmen" abgesehen ist die syntaktische zuordnung des Gerund zum Prädikatskomplex

45 Rủzička 66, S. 49; vgl, auch Kade 68, S. 610 
bei Zwischenstellung gesichert. Rủzicka 66 diskutiert diesen Distributionstyp nicht.

Für die folgenden Beispiele nehmen wir stellung des Gerund außerhalb der Modalität und der Reichweite der Negation des Prädikats an:

Gleichzeitig:

Berestov, priznavaja v suoem sosede nekotoroe sumasrodstro, odnako ne otrical v nem... dostoinstv (Gnevko 75,S.87) Ivan Kuz'mil, ocen' uvazaja svoju suprugu, vse-taki ni za to na suete ne otkryl by ej tajny, vverennoj emu po sluzbe (Paffen II,S.368)

A on, prjamoj, vysokij, stoja tverdo i krepko, protjagival k nim ruku i negromko, cetko govoril

(Rudnev 63,S.175)

Zeitliche Aufeinanderfolge:

Fedor ze Timofeevic byl inogo roda gospodin. Etot, prosnuvsis', ne izdaval nikakogo zvuka, ne sevelilsja i daze ne otkryval 81 az

(Rudnev 63,5.175)

Tetja Tosja, ostaviv masinu za vorotami, prisoedinilas'k nim (Adamec II 75, S.177)

Die Grenze zwischen vorzeitiger und vorzeitig-resultativer Bedeutung bis zur Gleichzeitigkeit kann dabei fließend sein: Čerez nedelju Michailov, razvernuv gazetu, uvidel i svoju, izrjadno sokraßcennuju, stat'ju (Gnevko 75, S.75)

Grundsätzlich besteht die Möglichkeit der Permutation an den Satzanfang :

Ivan, proscajas' so mnoj, vrulil mne poslanie na imja komendanta / Proscajas' so mojoj, Ivan vrucil ... = Kogda Ivan proscalsja so mnoj, on vrucil mne poslanie na imja komendanta (Paffen II,S.365)

Ja, buduci celovekom obscitel'nym, umel zivo rasskazyvat' = Čelovek obšitel'nyj, ja umel zivo rasskazyvat'

(Ananic 72, S. 34-35)

Die angeführten Beispiele zeigen die Stellung des Gerund auBerhalb der Negation und der Modalität des Prädikats. Das Gesamtbild denkbarer Varianten adverbialer Nebensätze verweist ebenfalls auf Analogie zu "Anfangsstellung mit Isolierung". Ebenso ist Koordinierung nicht grundsätzlich ausgeschlossen: 
Pavka, opasajas' privlec' vnimanie ryzeusogo, otvernulsjav storonu = Pavka opasalsja privlec' vnimanie ryzeusogo i otvernulsja $v$ storonu (Ananic 72.5 .61 )

Unmöglich ist Koordinierung (s.o.) wieder bei kond. Beziehung: Razve ty, imeja den'gi, ne tratil by ich = Razve ty, esli by u tebja byli den'gi, ne tratil by ich (Svetlik 70,5.269)

Im übrigen zeigt die Befragung von Muttersprachlern auch für die oben angefühten Belege in Zwischenstellung, die dem Typ "zwei voneinander verschiedene Handlungen" zugeordnet werden, daß im Einzelfall auch Relativsatzparaphrase nicht von vorneherein auszuschließen ist. Die Bewertung dieser Belege in der aktualen Gliederung macht Schwierigkeiten, solange nicht umfangreiche Untersuchungen zur Stellung in der Satzintonation vorliegen.

Eine Möglichkeit düfte die zusammenfassung von subjekt und Gerund zu einem in sich gegliederten Thema sein, das dem Prädikat als Rhema gegenübersteht:

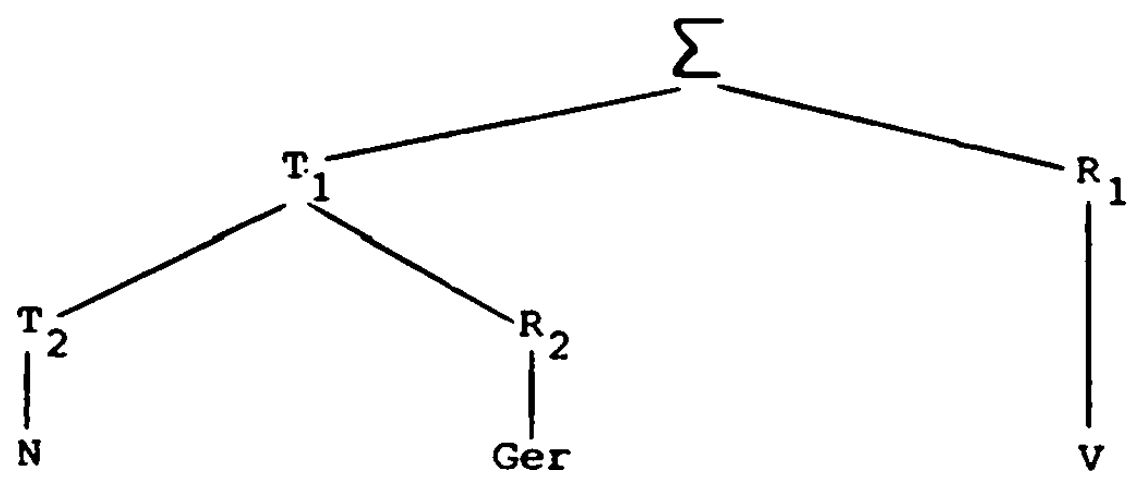

Entgegen Mikos Hinweisen zum slk. Gerund, wonach bei Stellung vor dem Prädikat in jedem Fall der Typ "zwei Handlungen" vorliegt, ist für das Russische auch bei stellung vor dem Prädikat mit dem Typ "eine Handlung mit ihrem Merkmal" zu rechnen, der meist als "modal" u.ä. umschrieben wird, ohne daß diese Gruppe dann scharf abgegrenzt würde. Relativsatzparaphrase ist nicht möglich. Das Gesamtbild semantischer Varianten bei Paraphrasierung/Ubersetzung durch adverbialen Nebensatz unterscheidet sich sich signifikant von den vorausgehenden Belegen für $\mathrm{Zwischen-}$ stellung und für Anfangsstellung (ohne Inversion): bei der 
Ubersetzung bietet sich am ehesten Ausdruck eines möglichst neutralen temporalen Verhältnisses durch indem an, gegebenfalls ohne zu. (während soll hier vorbehalten sein für ubersetzung zweier gleichzeitiger, voneinander verschiedener Handlungen.) Dabei ist nicht in jedem Einzelfall eine ausschließliche Klassifizierung möglich (s.o.). Dies beeinträchtigt nicht die Definition und Unterscheidbarkeit der strukturtypen selbst. sondern ist Indiz für die Ambiguität der Zwischenstellung. Bei Anfangsstellung ohne Inversion treten diese Probleme nicht auf.

Vgl. 2.B.:

Aksin'ja, spe ša, vygnala korov (Rudnev 63, S. 175) Drozki, mjagko sepeljavja kolesami, zakacalis' na vyboinach (Rudnev 63, S.175)

I on otryvisto, toropjas', tolno dogonjaja svoi mysli i s letu chvatajaslova, zagovoril ozizni v derevnes den'gami i bez dene 8

(Ruanev 63,S.181)

Vétojstat'e avtor, nestesnjajas', piset, cto...

(Paffén III, S. 209)

On, chitro ulybnuvisis', otvelal:... (Paffen III,S.209)

Hier setzt das Gerund die Gültigkeit der Prädikatshandlung voraus, das Prädikat kann nicht negiert werden, ohne daß auch das Gerund betroffen wäre.

Damit ergibt sich ein strukturell relevanter Unterschied in der aktualen Gliederung gegenüber den oben angefühten Belegen für "zwei voneinander verschiedene Handlungen". Jetzt gilt zusammenfassung des Gerund und des Prädikats in der aktualen Gliederung als in sich gegliedertes Rhema gegenuiber dem Subjekt als Thema; Relativsatzparaphrase ist hier nicht plausibel, ebenso nicht Permutation an den Satzanfang.

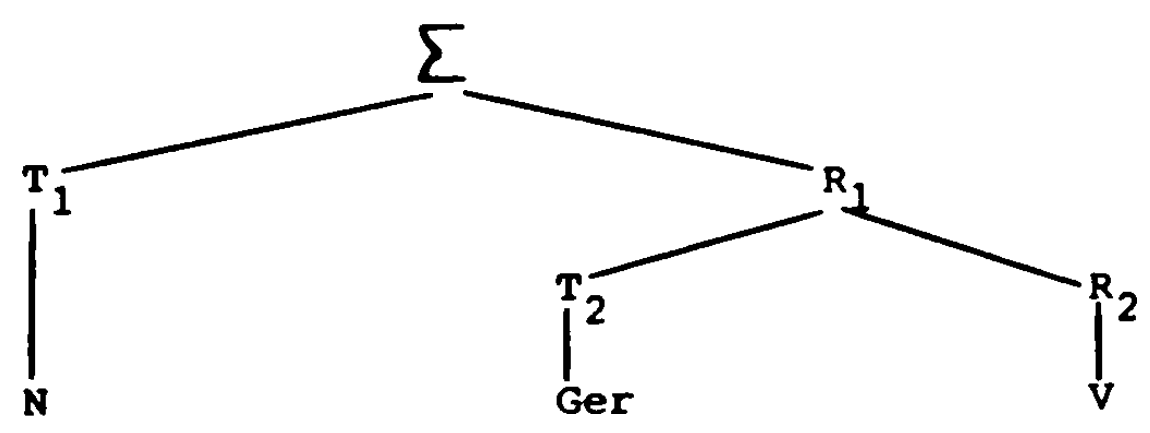


Dabei überwiegen in unserem Material bei diesem Funktionstyp ipf. präsentische Gerundien, während beim oben angeführten Typ "zwei voneinander verschiedene Handlungen" pf.. meist präteritale, Form vorherrscht.

\subsubsection{Skr. N + Ger + V}

"Zwei voneinander verschiedene Handlungen": ... zapitah ... sto oni Euvajuci stado rade (Biricz,s.94) (die Prädikatshandlung, nicht aber die Gerundialhandlung wird hier erfragt!)

otac radujuci se povede ih u svoj dvor (Musić 35.S.146) Vodenilar, videci Turcina da trei na konju, poplasi se

(Musić 35.S.146)

Auch im folgenden Beispiel, für das Belic Relativsatzparaphrase vorschlägt, liegt das Gerund "außerhalb" der Frage:

Kako ti, Jevreijn buduci, mozeł iskati ... = Kako ti, koji si Jevreijn, ... (Belić69,5.194)

Bei diesen Belegen tritt entweder überhaupt keine Isolierung auf, oder das Gerundialsyntagma wird durch Isolierung eingerahmt, oder diese steht nur zwischen Prädikat und Gerundialsyntagma. Nicht aber tritt in unserem Material Isolierung nur zwischen Subjekt und Gerundialkonstruktion auf, so daß Gerund und Prädikat zusammengefaßt bzw. N und Ger getrennt wirden. Dies entspricht der zugrundegelegten Struktur der aktualen Gliederung wie auch Elemente der Rückverweisung innerhalb der Gerundialstruktur. Auch die denkbaren Varianten der adverbialen Hypotaxe und die Unabhängigkeit von der Negation des Prädikatsverbs verweisen auf den Typ "zwei voneinander unabhängige Handlungen". Dieser kommunikativ-semantische Sachverhalt, nicht ein syntaktischer, liegt u.E. auch der verwirrenden Terminologie bei Musić zugrunde, der solche Belege als "appositiv" 46 bezeichnet mit der Begründung, die

46 Musić 35, S. 128, 147ff 
durch das Gerund angegebene Handlung sei gegenüber der verbalen als "prius" zu bewerten (gegenuiber "posterius" bei sogenannter prädikativer Funktion). Die Gerundien fungieren hier als Teil des Themas, nicht als Teil des Rhemas. Ein undifferenzierter Begriff der "adverbialen" Funktion, bei Belić begründet durch die akongruente Form des Gerund, erfaBt diese funktionalen Unterschiede nicht.

"Eine Handlung mit ihrem Merkmal":

Sinor sunce igrajuci zade, a jutroske potmolo izade

(Musić 35,S.146)

Covek placuci dode

(Belić 33/34,S.198)

Mati mu placuci odgovori

(Belić 69,S.193)

(Einrahmung des Gerund durch Prädikatssyntagma!)

In unserem Material finden sich nur präs. Gerundien, für die trotz Stellung vor dem Prädikat Geltung als "eine Handlung mit ihrem Merkmal" in Erwägung zu ziehen ist. Isolierung fehlt hier, ebenso Elemente der Rückverweisung: semantische Spezifizierungen über die Gleichzeitigkeit hinaus sind nicht zu motivieren, als Ubersetzung bietet sich am ehesten Partizipialkonstruktion an. Soweit Belić und Musić hier aufgrund der Wortstellung Interpretation als "zwei voneinander verschiedene Handlungen" nahelegen, fehlt eine weiterführende Begründung. 47 U.E. kann hier zumindest angenommen werden, daß die Setzung der Gerundialhandlung auch die Gültigkeit der Prädikatshandlung impliziert, also Negation des Prädikats, ohne daß das Gerund betroffen wäre, nicht möglicr ist. Ambiguitäten sind nicht ausgeschlossen. Gerade sie verweisen auf die konstituierende Relevanz der aktualen Gliederung für die Interpretation und das Nichtausreichen einer an der lexikalischen Semantik und dem außersprachlich Bezeichneten orientierten Klassifizierung.

Vgl. demgegenüber:

Carev sin cuvbi to, potrli brze boljeka koritu (Musić 35.5.147)

47 Musić 35, S. 143: Belić 33/34, S. $198 \mathrm{ff}$ 
8.2.3. Poln. N+I+Ger + I + V

Weiss 77, S. $329 f$ fieht bei grundsätzlich freier Permutierbarkeit die Zwischenstellung des poln. Gerund als stilistisch merkmalhafteste Variante. Belege für Zwischenstellung sind in dem von Weiss angefürten Material sehr selten, Anfangs- und Endstellung überwiegen bei weitem.

Vgl. z.B. mit Zwischenstellung, wobei die Isolierung konsequent auftritt:

Anna, nie czujac sie najlepiej, poszła wcześniej do domu

(Weiss 77, S. 329)

Charlotte Corday, stojac na chwiejnych nogach, rozpoczynaza monolog (Weiss 77.5.359)

Bogurodzica, bedqc pieśnia, mogła byc łatwo przekazywana ustnie (Weiss $77,5.359$ )

$\mathrm{Zu}$ weiteren Belegen mit bedqc vgl. a.a.O., S. $359 \mathrm{ff}$

Kongruierende und akongruente Form können durch einander ersetzbar sein und signalisieren den Gegensatz von nichtrestriktiv-attributiver und sekundärprädikativer Reduktion:

Król polski i ksqze ruski, obaj bedac/bedqcy piastami, uważali Ruśsa cześc dziedzictwa piastowskiego (Weiss 77,5.360)

Relativsatzparaphrase soll dabei für akongruente Formen grundsätzlich ausgeschlossen sein:

Charlotte Corday, stojac na chwiejnych nogach, rozpoczynaza monolog - sekundärprädikativ

Vgl.

Charlotte Corday, stojaca na chwiejnych nogach, rozpoczynała monolog - attributiv

(Weiss $77, \mathrm{~S} .359$ )

Demgegenüber ist für russische Gerundien in Zwischenstellung Relativsatzparaphrase nicht ganz auszuschließen und es ist für Partizipien in Zwischenstellung mit sekundärprädikativer Funktion zu rechnen (s.u.). 
8.2.4. Russ. $N+I+P+I+V$

Aus der Definition der russischen asymmetrischen Korrelation gegenüber der aksl. ergibt sich grundsätzlich für das isolierte Partizip als merkmalloses Glied die Möglichkeit zu untergeordnet prädikativer Funktion im Rahmen des Prädikatskomplexes. 48 Wenn das isolierte Partizip in $\mathrm{N}+\mathrm{I}+\mathrm{P}$ strukturelle Grundlage ist für die syntaktische Funktion des Partizips im Rahmen des Prädikatskomplexes bei $\mathrm{P}+\mathrm{I}+\mathrm{N}+\mathrm{V}$, ist $\mathrm{zu}$ fragen, ob nicht auch in Zwischenstellung das nichtrestriktive Partizip in potentieller Aquivalenz mit dem Gerund, d.h. untergeordnet prädikativ im Rahmen des syntaktischen Prädikatskomplexes fungieren kann, ob also die asymmetrische Korrelation nicht nur in Anfangsstellung, sondern auch in $\mathrm{z}$ wischenstellung realisiert wird.

Im Aksl. und in MS gilt für das merkmallose Glied der Korrelation, $\mathrm{P}_{k}$, die Möglichkeit derAmbiguität ${ }^{49}$ insbesondere in Zwischenstellung. Dabei hatten wir attributive zuordnung $2 u$ NP für $P_{k}$ insbesondere bei Restriktivität angenommen, während bei tichtrestriktivität Interpretation als nichtattributiv-peripherprädikativ nahelag.

Die Wahl zwischen Kurz- und Langform ist im Aksl. insbesondere bei Nichtrestriktivität relevant; bei Nichtrestriktivität schließt die Wahl der Langform Interpretation als peripherprädikativ aus. Im Russischen kann ein Motiv für die Wahl des Gerund darin bestehen, die bei Gebrauch des Partizips denkbare Interpretation als restriktiv auszuschließen.

Auch im Russischen sichert Restriktivität zuordnung des merkmallosen Gliedes $2 u$ NP. Bei Nichtrestriktivität und Zwischenstellung des akt. Partizips ist in der Regel untergeordnete Prädikativität im Rahmen des subjektskomplexes gegeben analog zum "einpoli-

48 S.0.: vgl. Rủzicka, SS, S. 20ff; Georgieva 68, S. 98ff

49 Dem entspricht es, wenn Pesikan 59, ohne explizit das Konzept der asymmetrischen Korrelation zugrundezulegen, attr. nichtrestr. Partizipialkonstruktionen als Ubergangserscheinung zum Funktionstyp "adverbial" einordnet (vgl.a.a.0.S.96): bei Nichtrestriktivität soll bis zu einem gewissen Grad Homonymie von attr. und adv. Funktionstyp vorliegen. 
gen" Bezug 50 des poln. aktiven Partizips.

Auf die grundsätzliche Möglichkeit der Ambiquität in zwischenstellung wurde hingewiesen in Zusammenhang mit Adverbialstrukturen sowie auch mit der nicht auszuschließenden Relativsatzparaphrase für Gerundien in $2 w i s c h e n s t e l l u n g$. Ambiguität der Zwischenstellung wird evident für das merkmallose Glied der Korrelation im Aksl. 51 Indiz für die potentielle vieldeutigkeit der $2 w i s c h e n s t e l l u n g$ ist außerdem die Unbestimntheit des Begriffes der Apposition, unter dem z.T. sämtliche untergeordnet prädikativen strukturen mit subjektsbezug subsumiert werden, 52 sowie die Möglichkeit für nichtrestriktive strukturen nach $N$, daß neben dem funktionstypkonstituierenden untergeordnet prädikativen Bezug auf die ibergeordnete subjektsphrase zusätzlich semantische Nuancen eines adverbialen Verhältnisses zur Prädikatshandlung mitverstanden werden. 53

Soweit für das Russische die Ambiguität des isolierten Partizips in $\mathrm{Zwischenstellung}$ ubergangen wird, hat das z.T. seine Ursache darin. daß das Problem der Korrelation nicht einbezogen wird.

Die Beispielsammlungen in Handbüchern führen $z$.T. nur die Fügung $\mathrm{N}+\mathrm{I}+\mathrm{P}$ an im Zusammenhang mit nachgestelltem isoliertem Partizip und schließen damit schon die Frage nach der Möglichkeit der zuordnung des Partizips zum Prädikatskomplex aus. Aber auch Rưzicka ${ }^{54}$, der die asymmetrische Korrelation zugrundelegt, nennt bei Zwischenstellung nur Funktion im Rahmen des Nominalsyntagmas (Ableitung iber "App" für nichtrestriktive isolierte postpositive Partizipien). Auch Ktizková, die für russ. Partizip die Funktion als doplnék bzw. predikativnyj opredelitel' nicht grundsätzlich ausschließt, nimmt bei Zwischenstellung unbescha-

$50 \mathrm{Vgl}$. Weiss $77, \mathrm{~s}, 352 \mathrm{ff}, 377 \mathrm{ff}, 382$

51 Umgekehrt hatten wir für die Langform in MS bei (u.a.) Zwischenstellung auch untergeordnet prädikative Funktion nicht ausgeschlossen, vgl. M.. S. $54 \mathrm{ff}$

52 So auch bei Birnbaum 60, S. 55ff; vgl. die Hinweise bei Weiss 77. S. $227 \mathrm{ff}, 352 \mathrm{ff}$, ders. 74, S. 201: vgl. Georgieva 68 , S. $103 \mathrm{zu}$ Potebnja

53 Bungarten 76.S.153ff: Rohrer 71.S.193ff zu Relativsätzen

54 Rủzicka 66 , S. $27 \mathrm{fE}$ 
det der Möglichkeit referentieller Synonymie für Partizip und Gerund unterschiedliche syntaktische Gliederung an. 55 attributiv:

Istobnye vopli, to narastajuscie, to nispadajuscie, stimali serdce. (Kłiźková 69.Synt.ch..S.118)

Bek, poterjavsij vsjakuju vyderzku, v panileskom strache govoril - tankach (Kíizková 69,synt.ch..S.119)

doplněk :

Istosnye vopli, to narastaja, to nispadaja, simali serdce (Kr̉izková 69. Synt.ch..S.118)

Bek, poterjav vsjakuju vyderzku, v panizeskom strache govoril o tankach (Kíizková 69, Synt.ch.,S.119)

Voraussetzung für Ersetzung des Partizips durch Gerund ist Nichtrestriktivität, die Ersetzung kann obligat sein bei pronominalem Subjekt. ${ }^{56}$ Die Frage ist, ob in solchen Fällen nicht morphologisch bezeichnet wird, was als möglicher Funktionstyp auch für das Partizip nicht auszuschließen ist. 57 wir schlagen vor, gemäß letztlich der Konzeption der asymmetrischen Korrelation auch für Partizipien bei Isolierung und Zwischenstellung funktionale Aquivalenz mit Gerundien in Zwischenstellung zuzulassen; Explikation erfolgt durch Paraphrase/Ubersetzung mit Koordinierung oder konjunktionaler Hypotaxe.

So wird von Muttersprachlern zumindest für den oben angefuhrten Satz: Bek, poterjavij ... auch Paraphrasierung durch adverbialen Nebensatz angefuhrt. Zuordnung zum Prädikatskomplex ist auch bei Partizip nicht auszuschließen z.B. bei den folgenden Beispielen:

Lesnye vzgor'ja, vse guśce lilovejuscie, uchodjat vdal'

(Kíizková 69, synt.ch.,S.119)

55 Vgl. Kłizková 69, Synt.ch.. S.115ff, 122

56 a.a.O., S.113ff, $121 \mathrm{ff}$

57 Vgl. Francuk 52, S. 8: "Takie obosoblennye pricastnye oboroty. kotorye svjazany po znaceniju so vsem predlozeniem, ne mogut rassmatrivat'sja kak sinonimy $k$ opredelitel'nym predlozenijam. Oni skoree sinonymicny deepricastnym oborotam ili drugim pridatoknym predlożenijam (casce - pridatoknym pridinnym)." 
Vgl .:

Lesnye vzgor'ja, vse guśde liloveja, uchodjat vdal' Ešelon, cut'-cut' zamedlivsij chod, vyšel na naplavnoj most Vgl.:

Ešelon cut'-cut' zamedlil chod i vysel na naplavnoj most

(Krizková 69, Synt.ch.. S.121)

Nakonec ja, snacala ne razdeljavij ego mnenie, vpolne soglasilsjas nim = Nakonec ja voolne soglasislsjas nim, chotja snatala ne razdelil ego mnenie (Adamec II 75,S.187)

Les, okontatel'no strachnuvijijs sebja ostatki notnogo mraka, vstavil vo vsem suoem zelenom velitii = Les, okoncatel'no strachnuv s sebja ostatki nocnogo mraka, vstaval ...

(Adamec II 75, S.186)

Eti slova, vosprinimavsiesja kak archaizmy, izbegalis'

(Adamec II 75, S.186)

Adamec klassifiziert derartige Belege explizit als "tavtosub- ektnoe dobavlenie"

Analog:

Moja mat', rodivşajas'za granicej, vse-taki chorošo govorit poanglijski

Mat', zabolevsaja vospaleniem legkich, nuzdaetsja v moej pomosci (Paffen II,S.356)

Vgl. mit Adjektiv in Zwischenstellung:

ob-jasnenie, jasnoe $i$ tocnoe, ne ubedilo ego (obwohl die Erklärung klar und deutlich war, iberzeugte sie ihn nicht.)

(Leitfaden, S.151)

Vgl. mit pass. Partizip:

Socinennye predlozenija, chotja logiceski i svjazannye meždu soboj, grammatiteski drug ot druga ne zavisjat

Vgl. gegenüber der Isolierung im Russischen im Cechischen konj. Hypotaxe mit Kopula:

Véty souŕadné, i kdyź jsou mezi sebou logicky spojeny

(Rưzicka 63,Typ.,S.842)

Für die Interpretation der oben angefuhrten Partizipien in Zwischenstellung ist zunächst $z u$ berücksichtigen, daß (wenn auch weniger plausibel) Relativsatz nicht mit absoluter sicherheit als Paraphrase auszuschließen ist, jedenfalls, solange man sich notgedrungen nur an der graphischen Repräsentierung orientiert. 
U.E. liegt hier allerdings zuordnung zum Prädikatskomplex vor, die durch Paraphrasen zu explizieren ist. Wird dies akzeptiert. so lassen sich die angeführten Beispiele zusammenfassen unter dem Typ "zwei voneinander verschiedene Handlungen", d.h. aufgrund der stellung in der aktualen Gliederung ist das Partizip nicht betroffen von der Negation des Prädikats. Ebenso wäre grundsätzlich möglich Permutation in die isolierte Position an den Satzanfang. Beide Kriterien sprechen gegen die Klassifizierung als zum TYp "eine Handlung mit ihrem Merkmal" gehörig. 58 Begründet wird die zuordnung des isolierten Partizips in Zwischenstellung $z$ um Prädikatskomplex durch die stellung in der aktualen Gliederung, 59 d.h. durch den rhematischen Charakter des Partizips.

Wir schlagen folgende schematische Darstellung vor:

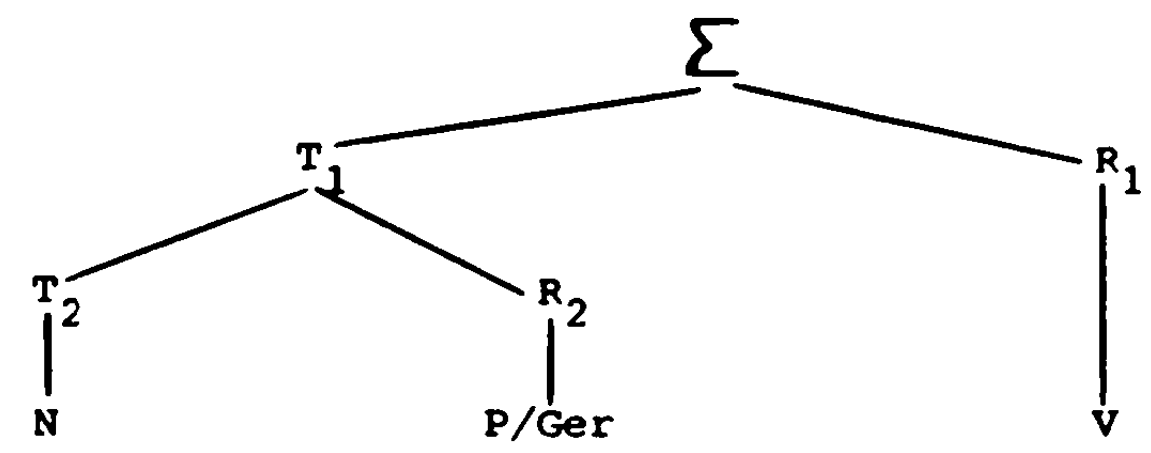

Der folgende Vorschlag hat nur den Charakter einer Vermutung und bedurfte umfangreicher Untersuchungen zur Textstruktur und ihrer Relevanz für die Konstituierung von satzgliedpositionen und Satzintonation; es wirde jedenfalls Seilers Bestimmung der "Apposition" als "Klasse" gegenüber dem Bezugsnomen als "Selektor" entsprechen, bei nichtrestriktiv-attributiven Partizipien

58 Dem entspricht auch, wenn Pesikan 59.S. 96 ff für nichtrestr. Partizipien, die er als Ubergangserscheinung zwischen attr. und adverbialer Funktion wertet, die hier allerdings nicht definierte "modale" Bedeutung ausschließt.

$59 \mathrm{Vgl}$. hierzu ausführlich Kornilov 64, ders. 67, ders. 71, der im Grunde die asymmetrische Korrelation rekonstruiert mit dem Merkmal der Nichtrestriktivität 
und bei Gerundien in Zwischenstellung, für die Relativsatz als Paraphrase vorgeschlagen wird, thematischen Charakter im Sinne des folgenden Schemas anzunehmen, was immerhin die wahl des Relativsatzes statt Koordinierung plausibel machen könnte $: 60$

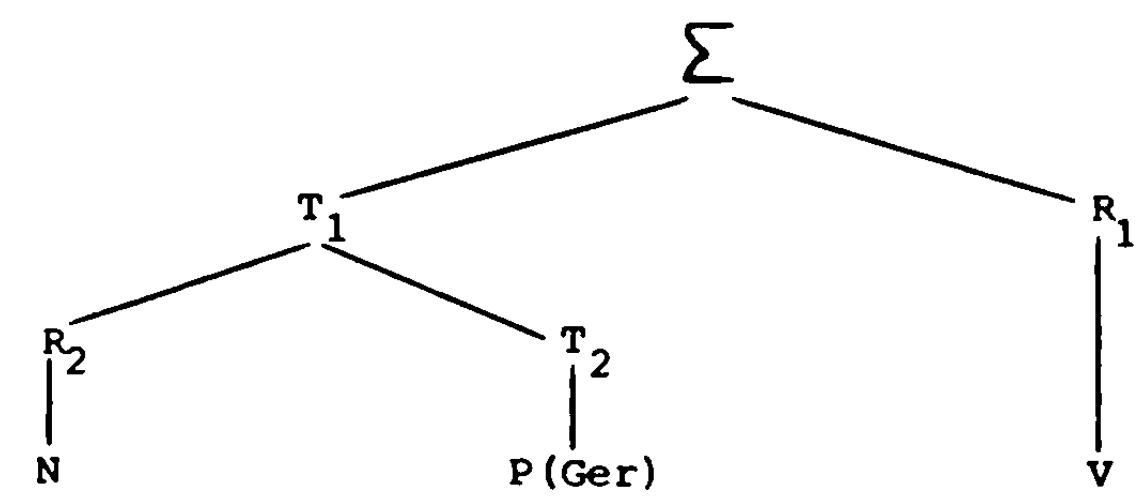

$\mathrm{Zu}$ beachten ist, daß bei den vorliegenden Beispielen für Partizip als $R_{2}$ in keinem Fall pronomen als subjekt fungiert.

$\mathrm{Vgl}$. demgegenüber attributiv:

Vse skorouleglis'. Ja, kurjaśtij, sidel na brevne pered saraem i govoril s Pavlom = I, who was smoking, was sitting... oder: I, as a smoker ... (Morison, S.53)

aber:

I, smoking $\ldots=\mathrm{Ja}, \mathrm{kurja}$, sidel $\ldots$

(Morison, S.53)

Ja kuril, sidja...

Vgl. zum Aksl. Rủzićka, SS, S. 69 (zur Ambiguität der Zwischenstellung für $P_{k}$ ): "... klar prädikativ ist das Kurzpartizip in analoger semantischer Variante nach Pronomina:"61

I nikbtoze pivo vetncha abbe chostetb novumu L 5.39 (SS,S.69)

Vgl. noch als Beispiele für russ. sekundärprädikative Gerundien nach Pronomen:

Ta, ozen'-to zadumavšis', smotrela na zakat (Gnevko 75,S.85)

$60 \mathrm{Vgl}$. in Zusammenhang mit der Struktur des peripherprädikativen Syntagmas im Aksl. zum Stellungsgegensatz $\mathrm{P}_{k}+\mathrm{N}: \mathrm{N}+\mathrm{P}_{k}$ Rủzicka, SS, S. 29

$61 \mathrm{Vgl}$. a.a.O.. S. 235: "Ein Kurzpartizip, das auf ein Pronomen bezogen ist, kann meist nicht als attributiv gelten. Allgemeine Güligkeit dieses distributiven Kriteriums kann jedoch nicht behauptet werden." 
Kogda ja, vernuvis' k statskoj zizni, priechal v lejpcig, ja ne zametil v moem druge kakich-libo peremen (F,S.193) I tu ze sekundu nad samoj golovoj razdaetsja gul, kotoryj, kak by podnimajas' vse vyse, postepenno usilivaetsja

(Ananid $72,5.56$ )

8.2.5. Aksl. $N+P_{k}+V$

Die Bedingungen für die Inversion des Subjekts mit dem Ergebnis der zwischenstellung des Partizips verweisen auf den textuellen zusammenhang von $N$.

Bei Anfangsstellung des $P_{k}$ kann dieses hervorgeheben werden, bei $\mathrm{Zwischenstellung}$ von $\mathrm{P}_{k}$ muß das subjekt hervorgehoben werden, insofern ist die Zwischenstellung merkmalhaft. 62 Die Hervorhebung des subjekts resultiert gewöhnlich aus Kontrast gegenüber dem vorangehenden oder folgenden Satz.

Auch wenn nach Ruzicka die unmittelbare peripherprädikative Beziehung zwischen subjekt und $P_{k}$ erhalten bleibt, ist die (peripher)prädikative Selbständigkeit des Partizips bei Zwischenstellung weniger ausgeprägt als bei Anfangsstellung. Anders als bei Anfangsstellung ist $N+P_{k}$ nur selten durch Punkt vom Prädikat getrennt, ${ }^{63}$ vielmehr ist jetzt die Abtrennung des Subjekts durch Punkt häufig und die latente Möglichkeit zur Verdeutlichung des Bezugs zwischen Partizip und Prädikat sichtbarer.

vgl. z.B.:

divleachq sq voi narodi ... farisei le slygavise rese

Mt $12,23 / 24$ (SS,S.31)

T. bo irods. posblave jets ioana $\operatorname{Mr} 6,17$ (SS,S.37)

Nikotole suetilunika vozego. vo krove polagaetr

$$
\text { L } 11,33 \text { (SS, S.70) }
$$

(Genitiv der Negation beim objekt infolge nikbtoze als Indiz für die syntagmatische Einheit von $N+P_{k}$ )

$62 \mathrm{Vgl}$. Ruzicka, SS, S. $29 \mathrm{ff}$

63 a.a.o.. S. $42 f f$ 
on b že vêdy ichn licemérne. reçe imh Mr 12,15 (SS,S.86)

Grundsätzlich ist aufgrund der Mcrkmallosigkeit des $P_{k}$ in Zwischenstellung auch mit der Möglichkeit der attributiven Funktion zu rechnen. 64 Auf derartige strukturen war bereits hingewiesen worden. Hier sei nur wiederholt, daß attributive Interpretation U.E. gegeben ist bei Kombination mit Langform, bei restriktiver Bedeutung und bei Funktion als schmückendes Beiwort.

Vgl als Beispiele für den Gegensatz $P_{k}: P_{l}$ in Zwischenstellung u.a.:

i Ėci chodestei (: chodeste) vrbchu ne videtb (SS,S.332)

i mozi drazestei (: drozeste) isa. rogachose emu bijosté

(sS, S. 332)

So weit aus dem Material bei Rúzicka ersichtlich, gilt fur alle peripherprädikativen $P_{k}$, solange sie sich auf $N$ stützen, also im Unterschied $z u$ Konstruktionen ohne Subjekt, daB Partizip und Prädikat zwei voneinander verschiedene Handlungen bezeichnen in dem Sinne, daß Negation des satzschließenden Prädikats möglich ist, ohne daß $P_{k}$ betroffen wäre: dies erweist zusätzlich die fortbestehende Gültigkeit des peripherprädikativen Syntagmas auch bei $\mathrm{N}+\mathrm{P}_{\mathrm{k}}$ im Unterschied $z \mathrm{u}$ russ. $\mathrm{N}+\mathrm{I}+\mathrm{Ger}+\mathrm{I}+\mathrm{V}$ mit Ambiguität hinsichtlich Bezug auf Prädikat : Bezug auf das Satzganze und im Unterschied auch $2 u$ aksl. $P_{k}+v$, wo das Fehlen des Subjekts immerhin die Tendenz zur Unterordnung des $\mathrm{P}_{k}$ unter $\mathrm{V}$ motiviert mit im einzelnen unterschiedlichen graduellen $\mathrm{Ab}-$ stufungen.

Vgl .:

mytare že i ljubodeice jesse emu vêrq. vy że vidév śe i ne raskaste se poslédo jeti emu vêrq Mt 21,32 (SS,S.31)

a mytaroiz dalece stoje. ne chotéase ... vizvesti

$$
\text { L } 18,13 \text { (SS,S.87) }
$$

In Analogie $z u \mathrm{P}_{k}+\mathrm{N}+\mathrm{V}$ schlagen wir fiir $\mathrm{N}+\mathrm{P}_{k}+\mathrm{V}$ als schematische Darstellung vor:

$64 \mathrm{Vgl} . \mathrm{a.a.0.,} \mathrm{s.} 43$ 


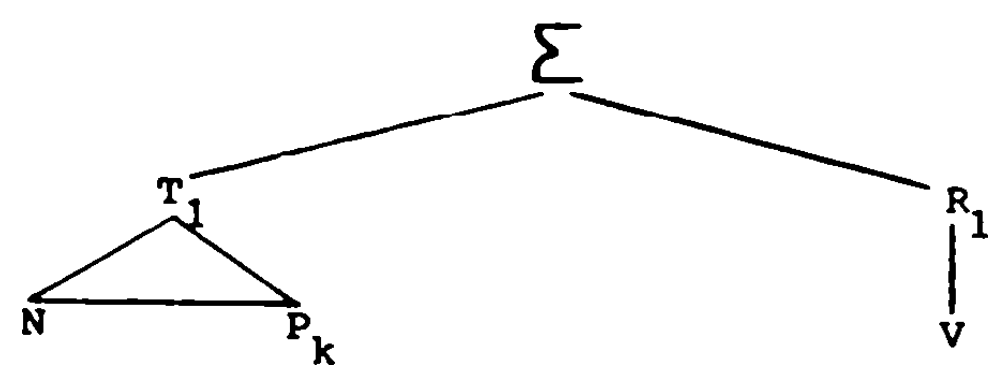

Auch in MS finden sich in Zwischenstellung $\left(N+P_{k}+V\right)$ Belege, 65 fiir die entsprechend dem Verhältnis im Aksl. noch eher peripherprädikatives Syntagma $\mathrm{N}+\mathrm{P}_{k}$ als Unterordnung unter die satzschließende Prädikation anzunehmen ist. Als typisch fur diese Interpretation werden Beispiele angesehen, bei denen sich das Partizip eng an das voraufgehende $\mathrm{N}$ anschließt und von $\mathrm{V}$ getrennt ist durch z.T. umfangreiche Einschübe.

$P$ und $V$ bezeichnen $z$ wei voneinander verschiedene Handlungen, das Partizip ist möglich auch bei negiertem $v$.

$\mathrm{Vgl}$ : :

I azo Radicn toi slisavo öt kralevstva, veselo i mogo vosesrodno upisach gospodé dubrovorkoi, mole i prose, da posılju... (MS, S. 241)

Gospodin. stefan. ... i öpčina ... chōteći i zlodece pokrépiti

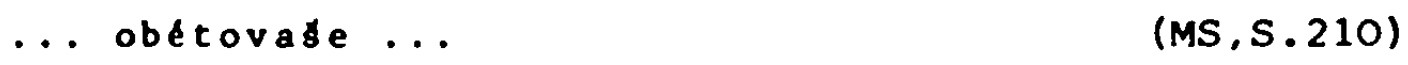

azn ze vesele se douseju i télom ó bozostronem dare ... Eto ti vozdamb, vladyko moi Christe, za vsa...? (MS,S.90)

Das Partizip ist nicht betroffen von der durch das Prädikat ausgedruickten Frage.

Die weit überwiegende zahl der Belege aus MS für Zwischenstellung wurde analog $2 u$ den oben angefürten bewertet. Es handelt sich meist um präteritale Partizipien zur Angabe eines Grundes. einer Voraussetzung, unter der die Prädikatshandlung stattfindet.

Die Entscheidung darüber, ob peripherprädikatives Syntagma $\mathrm{N}_{\mathbf{k}} \mathrm{P}_{\mathbf{k}}$ vorliegt oder ob die Unterordnung des $P_{k}$ unter die Hauptprädikation schon abgeschlossen ist, läBt sich angesichts der sehr verwickelten syntagmatischen Verhältnisse vieler Beispiele nur

$65 \mathrm{Vgl.M.}$. S. $30 \mathrm{ff}$ 
für den Einzelfall, nicht aber generell erörtern. U.E. sind beide Möglichkeiten vertreten. Jedenfalls ist mit der Möglichkeit der Auflösung des peripherprädikativen Syntagmas und der Unterordnung unter das Prädikat zu rechnen, wie die Beispiele (s.u.) für den Strukturtyp "Bezug nur auf das Prädikat" ("eine Handlung mit ihrem Merkmal") zeigen, die in MS auftreten, nicht aber in dem aksl. Material bei Rủžcicka.

Die strukturelle Möglichkeit andererseits des peripherprädikativen Syntagmas ${ }^{N}+P_{k}$ auch in $M S$ ist in Betracht $z u$ ziehen aufgrund der Belege für $\mathrm{P}_{k}+\mathrm{N}+\mathrm{V}$, für die analoge Auffassung $z u$ den aksl. Beispielen zugrundegelegt wurde. 66

Dem entspricht die "Einrahmung" des Subjekts azn durch die Partizipialkonstruktion in

... zogovorivo se $i$ azb sb bratijeju svojeju s episkoupy, zapisachb ... (MS,S.86; vgl. MS,S.76; vgl. prijem MS,S.213, vidervosi i MS,S.137)

Auch Koordination des $P_{k}$ mit dem Prädikat ist belegt: i sije prilo(zi)vosi i zapisavosi kraljevostvo mi svetoi Goré i molju (MS, S. 116)

Analog zum Aksl. hatte auch in MS bei $P_{k}+N+V$ ausschließlich der Funktionstyp "zwei voneinander verschiedene Handlungen" vorgelegen. Dies allerdings verweist auf die Signifikanz der Anfangsstellung, wie im Russischen auch, und noch nicht auf das Fortbestehen des peripherprädikativen syntagmas $\mathrm{P}_{k}+\mathrm{N}+\mathrm{V}$ als struktureller Voraussetzung für eine analoge struktur von $\mathrm{N}+\mathrm{P}_{k}$ $+\mathrm{V}$.

Wenn man peripherprädikatives $\mathrm{N}+\mathrm{P}_{\mathrm{k}}$ annimmt, liegt in jedem Fall der Typ "zwei voneinander verschiedene Handlungen" ( $P_{k}$ außerhalb der Negation des Prädikats) vor, bei syntaktischer Unterordnung von $\mathrm{P}_{k}$ unter $\mathrm{V}$ besteht die Alternative (anders als im Aksl.) zwischen "zwei voneinander verschiedene Handlungen" (s.o.) und "eine Handlung mit ihrem Merkmal" (s.u.). Die Unmöglichkeit der Verallgemeinerung entspricht dem der Entstehungszeit und der Provenienz nach heterogenen Charakter des

$66 \mathrm{Vgl} . \mathrm{M} ., \mathrm{S} .36 \mathrm{ff}$ 
sprachlichen Materials in MS sowie der Textart. Urkunden mit zugrundeliegenden überlieferten Schemata, die immer wieder durchbrochen werden.

Insbesondere in den häufigen Fällen, in denen auf das einleitende Nomen oder Pronomen eine ganze Serie von ihrerseits erweiterten Partizipien folgt, die die Vorgeschichte der in der Urkunde verbrieften Aussage des Prädikats schildern, erscheint es am einfachsten, den aus Nomen und Partizipien gebildeten Zusammenhang als in sich vielfach gegliederten thematischen Komplex aufzufassen, der dem durch das Prädikat mit seinen Erweiterungen gebildeten Rhema gegenübersteht.

Vgl.:

a sade mi knezb, vlastele i vsa öpkina ... smislivui smotrose ...videro... chtie ... utinismo ... (MS,S.239)

Für einige wenige, meist formelhafte Belege bei Zwischenstellung von $P_{k}$ scheint eher Interpretation als "ein Vorgang mit seinem Merkmal" plausibel, d.h.. nur das Satzganze kann negiert werden, nicht aber ist möglich Negation der Prädikatshandlung bei vorausgesetzter Gültigkeit der Partizipialhandlung; die Einheitlichkeit der durch das Prädikat und Partizip bezeichneten Handlung ergibt sich aus dem formelhaften Charakter und im Material belegten "Paraphrasen".

Vgl.:

... pripadaju mole se

gegenüber

azb... pripadaje molju ti se in:

jegoze blagostinou clovékoljubyé razoumbro azo gresoni stefan, velii kral., namstonyj gospodin॰ vose srbbiske zemle i Diöklije i Dalomatije i Travounije i chlomske zemlje, pripadaje molju ti se,gospodi ... (MS,S.9)

blagi ze bogb, ... suetto ofiju mojeju vozvrativo prosvęti me (MS.S.90)

Hier unterstellen wir Aufgabe des peripherprädikativen Syntagmas $\mathrm{N}+\mathrm{P}_{\mathrm{k}}$ und Unterordnung unter die satzschlieBende Prädikation (im Unterschied $2 u$ aksl. $N+P_{k}+V$ ).

Die semantische Klassifizierung wird gestützt durch die syntag- 
matische Struktur der Sätze. Typisch ist in diesen Fällen der durch vielfache Einschübe geschaffene Abstand zwischen $\mathbf{N}$ und $\mathrm{P}_{k}$ und die enge Nachbarschaft zwischne $\mathrm{P}_{k}$ und $\mathrm{V}\left(\mathrm{N}+\ldots \mathrm{P}_{k}+\mathrm{V}\right)$.

\subsubsection{Russ. Ger + I + V}

Ein grundsätzlicher Unterschied $2 \mathrm{u}$ den Strukturen mit $\mathrm{N}+\mathrm{I}+\mathrm{Ger}+$ $I+V$ besteht nicht: analog $z u$ aksl. $P_{k}+V$ ist in den allermeisten Fällen das Subjekt aus dem voraufgehenden Kontext zu erschlieBen bzw. das Gerund mit nachfolgendem Prädikat hat das subjekt gemeinsam mit einem voraufgehenden koordinierten Prädikat:

Vgl. z.B.:

Poobedav, Levin sel s knigoj v kreslo i, Citaja, prodolzil dumat' o svoej predstojastej poezdke

(Mulisch 75.S.196)

Als Beispiel für Ger+I+V können auch verschiedene Arten von unpersönlichen Konstruktionen genannt werden:

Rabotaja s gorjucim, nel'zja kurit'

(Svetlik 70.5.270)

Prolitav knigu, sleduet vernut' ee v biblioteku

(AG 70,5.645) 67

Vzjavsis'za guz, ne govori, tho ne djuz

(Ananic $72, \mathrm{~S} .645$ )

Odnako, boltaja s toboj, gribov ne naberes' (Rudnev 63,5.178)

Für die oben angeführten Belege wie für die folgenden wird der Typ "zwei voneinander verschiedene Handlungen" infolge Nichtbetroffenheit durch die Negation des Prädikats angenommen:

Dver'. propustiv Natal'ju, pritvorila Aksin'ja. Pritvoriv, stala sredi komnaty, sunula ruki za belyj perednik (Rudnev 63,5.174) on vinovat $v$ tom, cto, somnevajas' v cestnosti svoego sotrudni$k a$, doveril emu zavod

(Isacenko $75, \mathrm{~S} .332$ )

Aufgrund der stellung auBerhalb der Reichweite der Negation und denkbarer semantischer Varianten ${ }^{68}$ schlagen wir in Analogie zu

67 a.a.O. werden derartige Belege inkonsequenterweise nicht als Determinant gesehen, sondern als Umstandscharakteristik

68 Zum aksl. $P_{l}+V$ vgl. auch die Aufzählung semantischer Varianten bei Rủžlcka, SS, S. $59 \mathrm{ff}$, die unter "zwei voneinander verschiedenen Handlungen"einzuordnen ist; Rứzicka nennt zur Explikation da, weil, obwohl, nachdem 
den oben angeführten Belegen folgende schematische Darstellung vpr, wobei das zu ergänzende subjekt in klammern gesetzt ist:

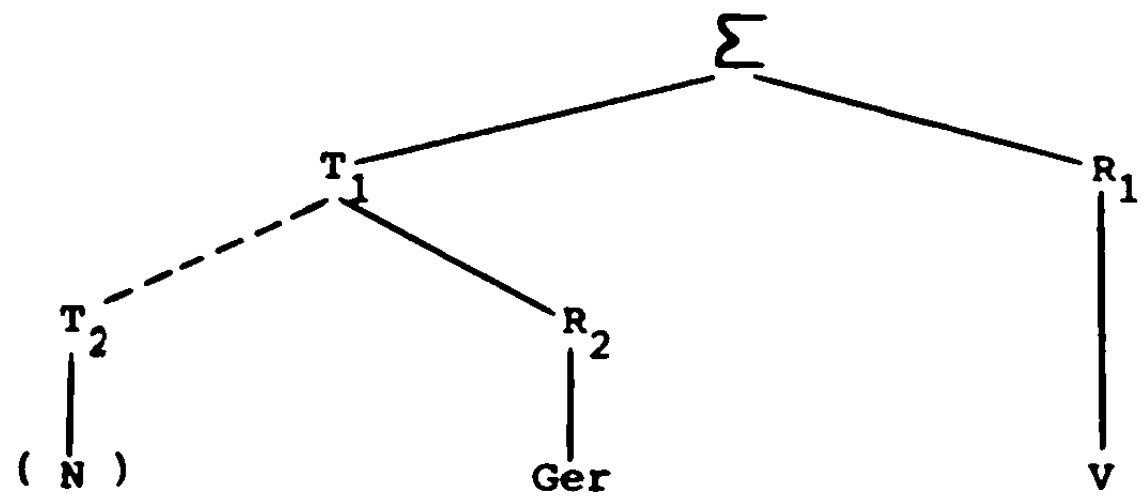

alternativ:

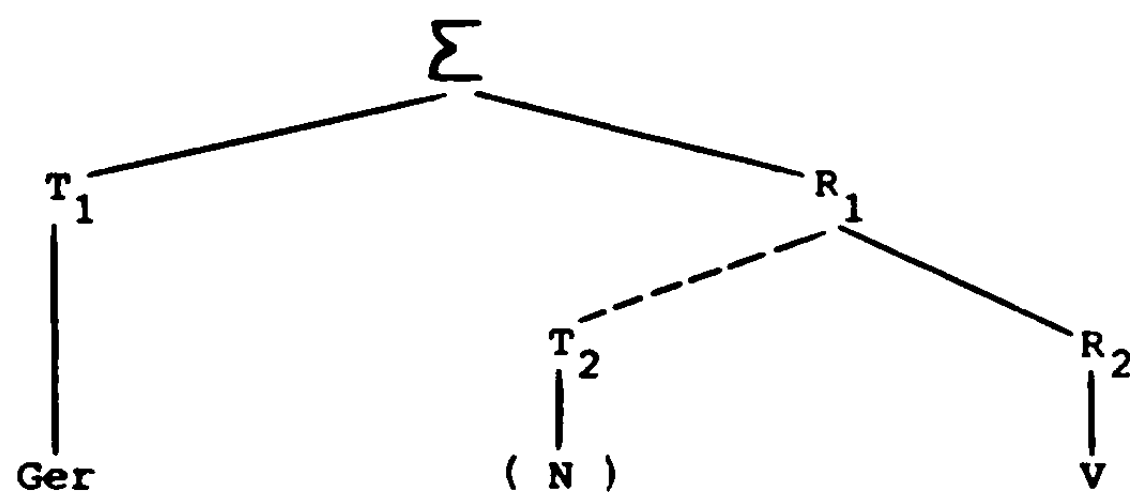

In jedem falle tritt als Realisierung von $T_{1}$ nur Ger auf, das vom Prädikat unter $R_{1}$ getrennt ist.

Vgl. hierzu auch:

Velosipedist... promealsja... i, proechav ebce nemnogo vpered, (on) vdrug ostanovils ja

Vgl. demgegenüber die folgenden Beispiele, für die immerhin unterstellt werden kann, daB mit der Gerundialhandlung die Gültigkeit auch der Prädikatshandlung vorausgesetzt ist ("eine Handlung mit ihrem Merkmal"):

On vernulsja, i poniziv golos, skazal: ... = Er kam zurück und sagte wit leiser stimme...

(Paffen II,S.375)

Sala nachmurila brovi, sderzivaja svoe ofivlenie, pomoleala, i ser'eznym golosom, radostno ulybajas', sbivtivo progovorila...

(Rudnev 63,S.176) 
Schematisch:

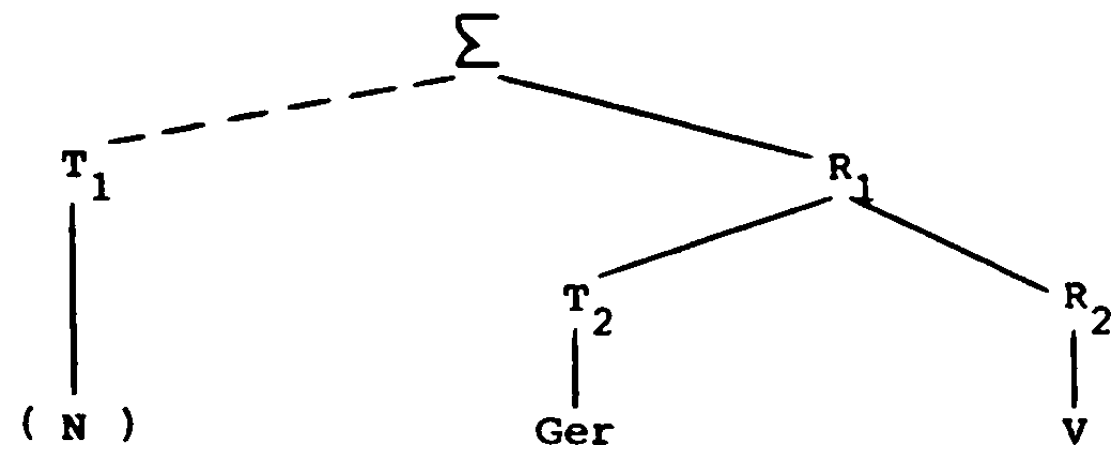

Beide Funktionstypen (Unterordnung unter $T_{1}$ und unter $R_{1}$ ) sind im folgenden Satz realisiert:

Milja vyucila vse naizust', no)volnujas' i ne doverjaja sebe, ona, stoja posredi komaty, esce raz povtorjaet tekst roli

(Paffen II,S.367)

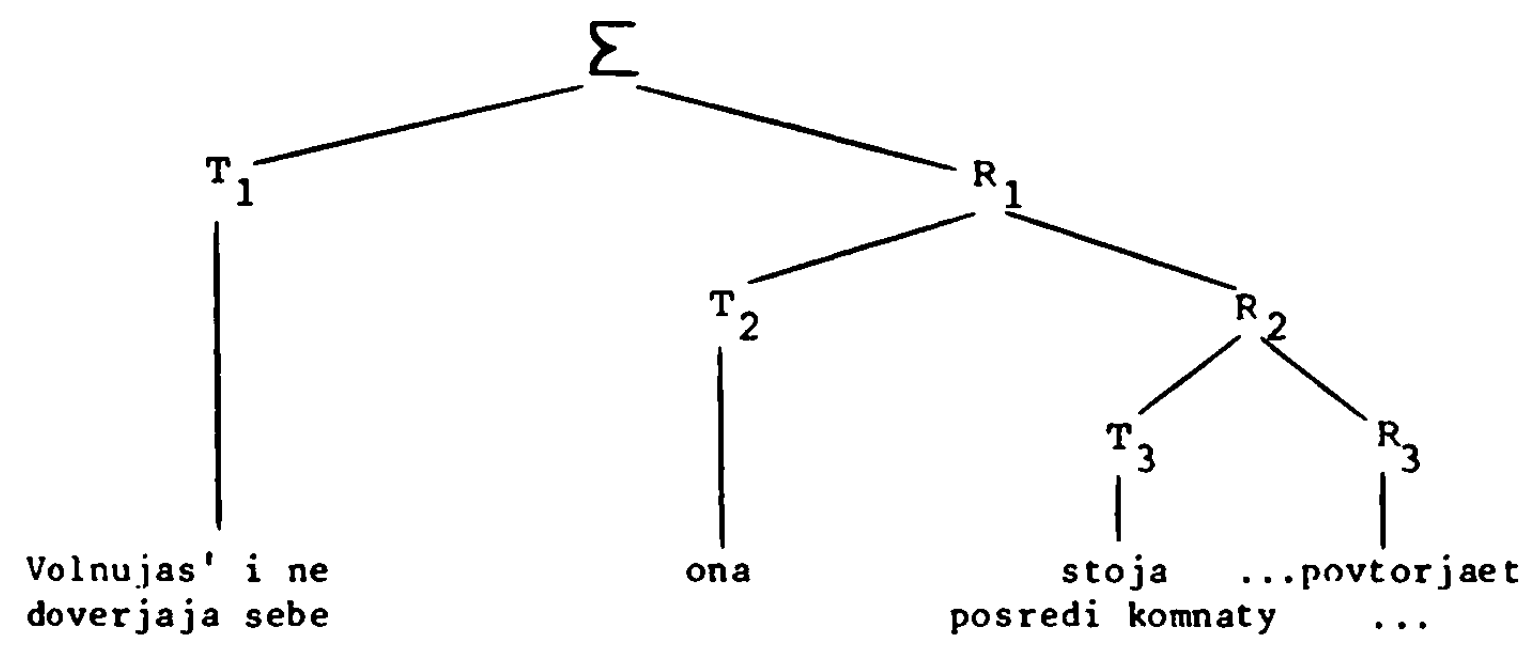

Maßgeblich für die unterschiedliche Interpretation der beiden Gerundialkomplexe ist ihre gemeinsame Arwesenheit im Satz; in ona, stoja posredi komnate, esce raz povtorjaet tekst roli könnte man auch die Möglichkeit der Negation des Prädikatsverbs bei Gültigkeit des Gerund annehmen. Eindeutig und ausschließlich wäre diese Interpretation gegeben bei:

stoja posredi komnaty, ona esce raz povtorjaet tekst roli.

So gut wie nicht ist in dieser Distribution das akt. Partizip belegt; vgl. allenfalls:

Sli celyj den', i tol'kok vezeru, utomlennye i progolodaviesja, dostigli slobody

(Rudnev 63.5.163) 
8.2.7. Skr. Ger + V

Die Isolierung wird nicht konsequent verwendet. Dabei sind Belege ohne Isolierung relativ häufiger bei Ger+V als bei $N+G e r+V$. Erweiterung des Gerund ist im vorliegenden Material eine notwendige, aber nicht hinreichende Bedingung für Isolierung. Činéci ovo necete pogresiti (Belić 69,S.193)

Zato im govorim u pricama, jer gledajuci ne vide, i cujuci ne cuju niti razumiju.

(Belić 69,S.193)

On siromah pode malo, ali ne mogavis srcu odoleti, vrati se natrag.

(Musić 35, S.147)

Für die angeführten Belege wird wieder Interpretation als "zwei Handlungen" angenommen mit der Möglichkeit der Negation des Prädikats unabhängig vom Gerund (s.0.). Diese Interpretation wird auch gestützt durch Elemente der Rückverweisung beim Gerund (vgl. tako, ovo, on)

Vgl. demgegenüber:

suteci je pojeo svoju veceru=sutke je pojeo (Teźak-Babić, S. 248)

Satzbezug und Bezug nur auf den nachfolgenden Infinitiv liegen im folgenden Beispiel vor:

Sobravis se, poceo je polagano i gotovo sapcuci govoriti (Hamm, S. 104)

8.2.8. Aksl. $P_{k}+V$

Auch wenn das Subjekt grundsätzlich aus dem Kontext erschließbar ist und Rủzicka auch für diesen Distributionstyp aufgrund der Gilligkeit der nominalen Kategoriendes $P_{k}$ die Spezifik der alten peripheren Prädikativität im Gegensatz zur russischen

$69 \mathrm{Vgl}$. Rúzicka, SS, S. 48: "Es liegt in der Eigenart dieser subjektlosen Partizipialkonstruktion, daß sie meist einen Satz, dessen subjekt sie teilt, als vorläufer hat und sich an ihn durch die Konjunktion $i$ anschließt. Die Enge dieser Verbindung ist... nicht immer sicher feststellbar. Die Konjunktion i ist ein fast ständiger vorausgehender Begleiter der subjektlosen Partizipialkonstruktion."; vgl. a.a.0., S. $43 f f, 50$ 
sekundären Prädikativität erhalten sieht (d.h. nicht Unterordnung unter $V$, sondern peripherprädikatives Syntagma, das als Ganzes auf $v$ bezogen ist), es zeichnen sich doch Gliederungsmöglichkeiten ab, die dann für die Unterordnung des Gerund unter die satzschließende Prädikation im Russischen kennzeichnend sind ${ }^{70}$ und $i m$ Aksl. anhand von $P_{k}+N+V$ sowie anhand von $N+P_{k}+V$ nicht zu begründen waren, ebenso in MS nicht für $\mathrm{P}_{k}+N+V$ und allenfalls an einigen Belegen fur $\mathrm{N}+\mathrm{P}_{\mathrm{k}}+\mathrm{V}$.

Bei $\mathrm{P}_{k}+\mathrm{V}$ finden sich auch im aksl. Material Belege mit unterschiedlicher Reichweite der Negation bzw. der Modalität des satzschließenden Prädikats. Das heißt: im Falle von $\mathrm{P}_{k}+\mathrm{V}$ besteht die Möglichkeit der Ambiguität hinsichtlich "eine Handlung mit ihrem Merkmal" : "zwei voneinander verschiedene Handlungen"; die $P_{k}$ innerhalh der Reichweite der Negation bzw. der Modalität des Prädikats verweisen auf die spätere Unterordnung des Gerund unter die satzschließende Prädikation.

Ublich ist für den Typ "Einheit der Handlung" im Aksl. Postposition: der Hinweis auf den Charakter als "feste Wendung" oder die Sachbedeutung ${ }^{71}$ von $v$ und $P_{k}$ liefert allenfalls Indizien, nicht jedoch ein strukturelles Kriterium für die Begründung dieses Funktionstyps.

Vgl. zum Strukturtyp "zwei voneinander verschiedene Handlungen" Z.B.:

tbgda vidé ijuda... éko osqdişg i. raskaevs se vnzvrati. 30 snrebrnnikn. archieroms... i povrbgh e vincraknve otide. i ośndr vinzvesi se. Mt $27,3 / 5$ (SS, S. 45)

Sego radi prithcami imu glja. éko videste ne videtb. i slyşste ne slysetr. ni razumejotr.

Mt 13,13 (SS,S.94)

Das Prädikat ist negiert, jedoch nicht das Partizip.

70 Vgl. Rúzicka, SS, S. 44: "Freilich steht außer Zweifel, daß der subjektlose Typ am meisten zur Annäherung des Partizips an das Verb drängt: gefördert werden kann diese Annäherung durch die Sachbedeutung des Partizips im Verhältnis zu der des Verbs. Endpunkt der Annäherung ist in den Einzelsprachen der Anschluß an das Prädikat als dessen "Erweiterung"."

$71 \mathrm{Vgl}$.a.a.o.. S. 56 
Moleste ze se ne licho glete Mt 6.7 (SS,S.99)

"Das Partizip ist nicht vom imperativischen Modus erfaßt"

(SS, S.99)

bodite ubo ... da ne prisodb vo nezaépq obrętetb vy supeste

Mr $13,35 / 36$ (SS,S.74)

"Negiert ist das Finden, nicht das Kommen"

(SS, S. 74)

"eine Handlung mit ihrem Merkmal":

Vgl : :

i potostavose solete

L 19,6 (SS, S.56)

i pridq podviguse

L 2,16 (SS,S.183)

i teprse ubijgto i

L $18,32 / 33$ (SS, S.72)

Das Partizip liegt innerhalb der Reichweite der Negation bzw. der Modalität des Prädikats:

i vozumetb slovo oth srbdoca ichb. da ne véry imsse speni bqdqtb L 8,12 (SS, S. 74)

i gla emu vosé si damo ti. aste pads poklonisi mi se

Mt 4,9 (SS, S.75)

Sodnse pokažite se iereomb

L 17,14 (SS, S.78)

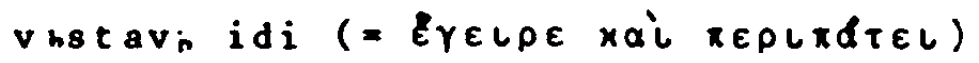

Mt 9,5 (SS, S.79)

Vgl. analog in MS:

"zwei Handlungen":

vas molju, bratja moja ljubimaja, prodetaje se prastaite

(MS, S. 181)

"eine Handlung" :

molju ke vy, ötoci i bratije... aste tto boudetr... pogréśno

... ispravljajuste i prochodeste cotete i blagoslovite, a ne

klonete

(MS, S. 84)

8.3.

Endstellung mit Isolierung im Russischen

Auf die distributionelle Relevanz des Gegensatzes der Wortstellung vor : nach dem Prädikat für Gerund bzw. für $P_{k}$ wurde bereits hingewiesen.

Der Neigung zur Ubereinstimung von Wortstellung und Zeitenfolge entspricht das tberwiegen von pf. prät. Formen in Präposition und präs. ipf. Formen des Gerund (bzw. von $\mathrm{P}_{k}$ ) nach dem Prädikat; doch ist dies eben nur eine statistische Tendenz und nicht 
eine "Regel". Grundsätzlich läßt sich nur wiederholen, daß Tempus und Aspekt zwar relevant sind für die Klassifizierung auch postponierter Partizipien und Gerundien, daß aber eine durchgängige Klassifizierung nach Kriterien der Zeitenfolge auch für postponierte strukturen bis anhin nicht möglich ist. Dies motiviert, analog zu präpositiven Gerundien, die Frage nach allgemeineren Kategorien der Differenzierung des Prädikatskomplexes und nach der Signifikanz der Postposition selbst.

Der Tendenz zu stärkerer Unterordnung unter das satzschlieBende Prädikat in Postposition mit gradueller Reduzierung des peripherprädikativen Eigenwertes entspricht es grundsätzlich, wenn Miko für das slk. Transgressiv bei Präposition den Typ "zwei von einander verschiedene Handlungen in Beziehung $z u$ einander" vermutet gegenüber dem Typ "eine Handlung mit ihrem Merkmal" bei Postposition. Allerdings darf auch für das slk. die Eindeutigkeit des Stellungsgegensatzes bezweifelt werden.

Im Aksl. gilt überwiegend, jedoch nicht ausschließlich, bei Präposition der Typ "zwei voneinander verschiedene Handlungen" (als Folge letztlich der spezifischen struktur des peripherprädikativen Syntagmas); bei Postposition ist, wie das Material zeigt, mit Ambiguität zu rechnen.

Für das Russische hatten wir auch bei Präposition (außer bei $\mathrm{P} / \mathrm{Ger}+\mathrm{I}+\mathrm{N}+\mathrm{V}$ ) den Typ "eine Handlung mit ihrem Merkmal" unterschieden. Am Material erweist sich ebenfalls die Ambiguität der Postposition. Indiz hierfür ist auch, daß bei Präposition die Nichtisolierung nichtadverbialisierter Gerundien so gut wie ausgeschlossen ist, während bei Postposition mit isolierten und nichtisolierten strukturen $\mathrm{zu}$ rechnen ist.

Zur Illustrierung der Ambiguität der Postposition für Adverbialstrukturen sei erinnert an Beispiele wie:

Er schlägt seine frau nicht, weil er sie liebt (Immler,s.95)

d.h.: a) weil er sie liebt, schlägt er seine Frau nicht oder: b) nicht weil er sie liebt, schlägt er seine Frau mit den abstrakten Repräsentationen: 
a)

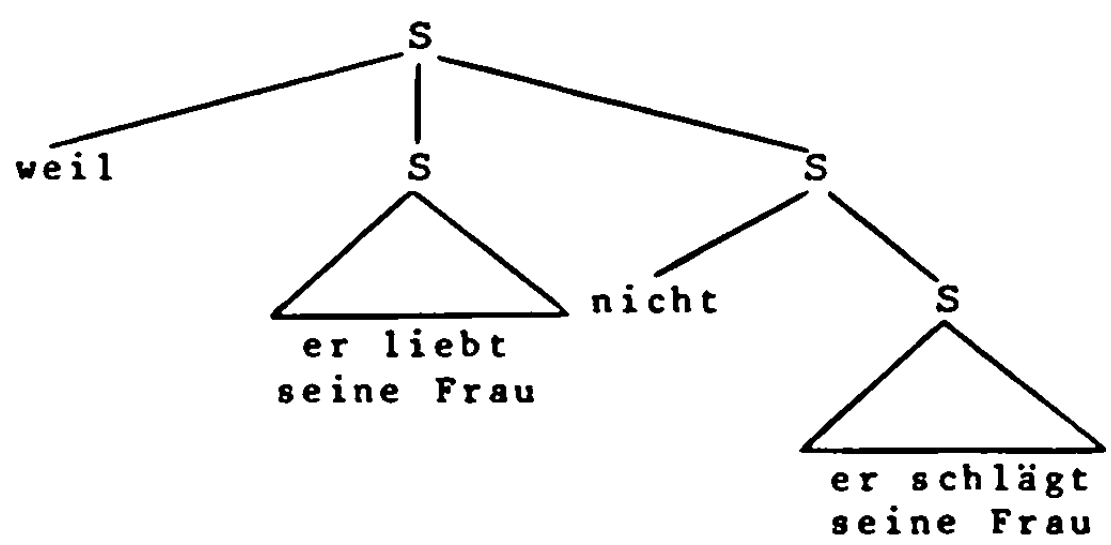

b)

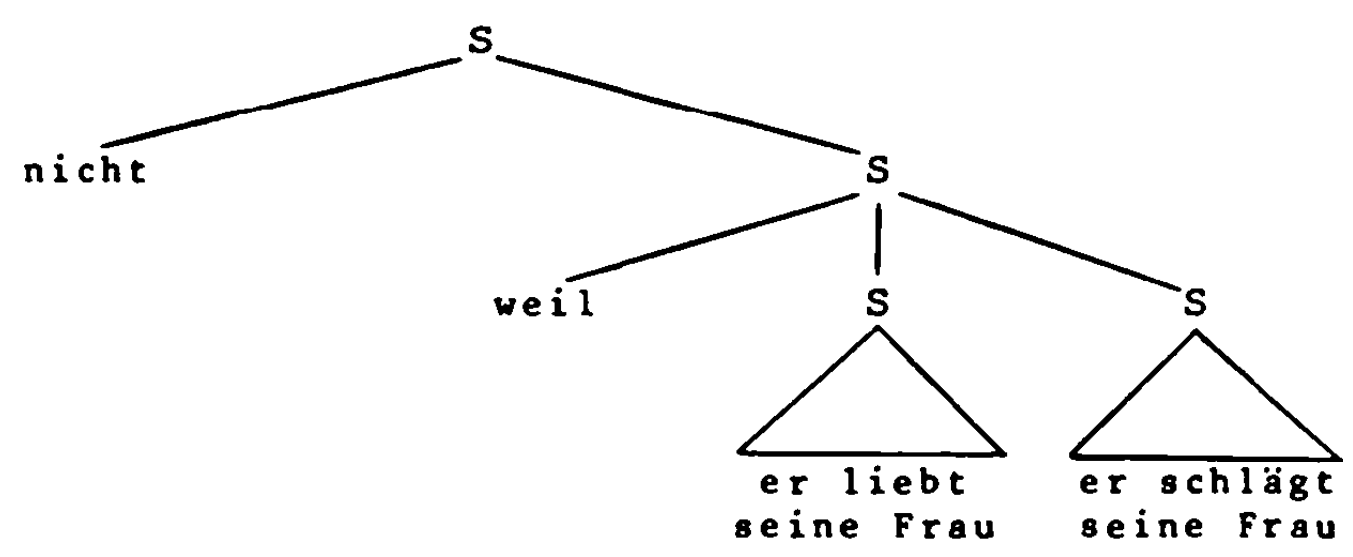

Zur unterschiedlichen Negierbarkeit des Prädikats auch bei

Strukturen mit impliziter subjektsidentität vgl. nochmals:

Er schimpte wutentbrannt: $?$ er schimpt wutentbrannt nicht

(Bartsch 72,S.143)

Er kehrt verärgert zurück: er kehrt nicht verärgert zurück:

er kehrt verärgert nicht zurück (Bartsch 72,S.143)

Petra kocht, wobei sie salz verwendet (Bartsch 72,5.173)

* Petra kocht nicht, wobei sie salz verwendet (Bartsch 72,5.173)

Petra kocht (nicht), während sie salz verwendet (Bartsch 72,S.173)

Die Möglichkeiten. Partizipial- und Gerundialstrukturen analog zu differenzieren, sind noch kaum untersucht. 72

Für das Russische verweist Rửicka 73 auf die semantische Re-

72 Vgl. Sgall in Danes 74 (ed.), S. 65 
levanz der Postposition, ohne sie zu präzisieren ${ }^{73}$, wie ja schon bei Rúzicka 66 das prädikatsfernere "Prädsec" in Beispielen mit Anfangsstellung, das prädikatsnähere "Advb" in Endstellung auftrat.

Ausfuhrlicher erörtert Weiss 77 am Beispiel poln. Gerundialkonstruktionen die Ambiguität der Postposition. Dabei greift er implizit auf eine Differenzierung zurück, die er auf der syntaktischen Ebene zunächst abgelehnt hatte und die sich u.E. begründet durch die stellung der fraglichen strukturen auf der Ebene der in der Kommunikation konstituierten sprachlichen Bedeutung.

Hier werden u.a. fokussierte strukturen unterschieden:

Profesor X wykłada (tylko, właśnie) siedzac (Weiss 77,5.301)

On szedz kulejac

(Weiss 77.S.276)

Daß bei derartigen Belegen Paraphrasierung weitgehend ausgeschlossen sein dürte, inbesondere Koordinierung nicht möglich ist, ist bedingt eben durch die Fokussierung und nicht durch die Tatsache der "Hyponymie". 74

Von Fokussierung zu unterscheiden ist die Funktion als "Kulisse", d.h., die Gerundialhandlung wird nicht eigentlich behauptet, sondern stillschweigend als wahr vorausgesetzt. Das Gerund liegt außerhalb der Frage- oder Befehlsmodalität des Prädikats:

73 Vgl. Rủżicka 73, S. 470, Anm. 18; zur Interdependenz von Tempus, Aspekt, dem morpholog. Gegensatz Partizip:Gerund, lexikalischer Bedeutung und Wortstellung vgl. ders. 59, S.544ff, ders. 66, S. $47 \mathrm{ff}$

74 Dies entgegen Weiss 77, S. 276; unsere Kritik richtet sich nicht gegen die Interpretation, sondern gegen die Allgemeinguitigkeit der am außersprachlich Bezeichneten orientierten Begrüindung; es ist mit Belegen $z u$ rechnen, bei denen zwar Fokussierung, nicht aber Hyponymie vorliegt. Die Problematik der referentiellen orientierung wird u.E. auch deutlich bei Weiss 77, S. 323, wonach Koordinierung durch $i$ eher 2 wei wesensverschiedene Sachverhalte als zwei Aspekte ein und desselben Sachverhaltes miteinander verbinden soll. Es ist U.E. nicht ersichtlich, daß durch die Koordinierung anders als bei gerundialer Konstruktion die beiden (!) Sachverhalte als "zwei getrennte Entitäten" dargestellt sind. Vgl. hierzu ein aksl. Beispiel, in dem Koordinierung gerade zur Sicherung der Einheit eines Vorgangs verwendet wird: sbpsi se samw.i

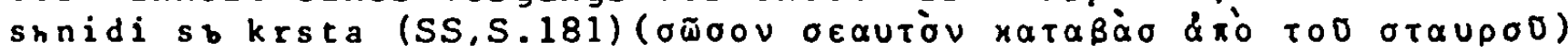


Czy wpadniesz do mnie, wracajac z uczelni? (Weiss 77,5.304) Wpadnij do mnie, wracajsc uszelni! (Weiss 77,5.304)

Vgl. demgegenüber:

Czy profesor X wakłada siedzac? (Weiss 77,5.301)

Wykzadajsiedzac! (Weiss 77,5.301)

Der Kulissenfunktion entspricht die Unmöglichkeit der Paraphrasierung durch Koordination und die Möglichkeit der Paraphrase durch Temporalsatz:

Czy wpadniesz do mnie, jak bedzies wracaz z uczelni?

(Weiss $77,5.304$ )

Wpadnij do mnie, jak bedziesz wracaz z uczelni!

(Weiss $77,5.304$ )

Weder Fokussierung noch Kulisse soll bei den folgenden Beispielen für Postposition vorliegen, wo Koordinierung, nicht aber konjunktionale Hypotaxe als Paraphrase angefuhrt wird. 75 Im Unterschied zu "Kulisse" wird hier das Gerund dem Bereich des regierenden Prädikats zugeordnet. Ambig hinsichtlich dieser beiden Interpretationen ist z.B.:

Ksigdz kazaz mi modlic sie idqc do ottarza

- Idz do ottarza i modl siq!

- Modl sie, jak bedziesz szedz do ottarza! (Weiss 77,5.305)

8.3.1. Russ. $N+V+I+G e r$

Entgegen z.B. Kłlzková ${ }^{76}$ sehen wir auch für isolierte russische Gerundien in Postposition die Notwendigkeit zu semantischer Differenzierung. So sehr es zutrifft, daß in diesen Fällen die Isolierung als Grenzsignal der aktualen Gliederung fungiert, u.E. ist auch hier zu unterscheiden zwischen Bezug auf das Satzganze (umschrieben als "zwei voneinander verschiedene Handlungen") und Bezug nur auf das Prädikat ("eine Handlung mit ihrem Merkmal"). Ausgeschlossen allerdings ist infolge Isolierung Fokussierung

$75 \mathrm{Vgl}$. Weiss 77, S. 302ff, $322 \mathrm{ff}$

76 Krízková 69, Po, S. 27 
des Gerund, d.h. Funktion als alleiniges rhematisches Zentrum im Satz. Aber auch Nichtisolierung ist nicht eindeutig (s.u.). Für die folgenden isolierten russischen Gerundien in Postposition wird Klassifizierung als "zwei voneinander verschiedene Handlungen" infolge Bezug auf das Satzganze vorgeschlagen. Bei den hier aufgeführten ipf. Belegen wird Gleichzeitigkeit zugrundegelegt. Sie geben den Hintergrund an, vor dem sich die Handlung des satzschließenden Prädikats vollzieht; im einzelnen können dabei z.B. kausale, konditionale, instrumentale Variante (weil, wenn, dadurch,daß) vermutet werden neben der Grundbedeutung der Gleichzeitigkeit (während, als): eine Klassifizierung ist mit der Unterscheidung dieser Varianten nicht $z u$ begrüden, sondern allenfalls durch ihre Zusammenfassung als Gruppe möglicher Realisierungen des oben angefuhrten Typs der Konnexion.

Ja vzdrognul ot neozidannosti, ne rasstityvaja v ètom gluchom ugolke vstretit'sja s ljudmi = Ja vzdrognul..., tak kak ne rassityval vétom gluchom ugolke vstretit'sjas ljudmi

(Ananid 72,S.62)

Ja ne mog vam napisat', ne znaja vašego adresa

(sint.r.j.74,s.137)

Da, u menja drotit ruka, kogda ja pisuéti stroki, i vse ze ja podeljus' tem, cto znaju, spokojno i prosto, - utesajas' do nekotoroj stepeni mysl'ju, kotoruju ja uze vyskazyval

(F,S.189)

Vgl. auch mit unpersönlichen Konstruktionen:

Kak te nam idti, znaja, cto perepravy net (AG 70,5.645) Ja, govorit, zamut neproc'; tol'ko gde ego najdes', doma-to sidja

(Morison, S.31)

Das Gerund liegt außerhalb der nichtassertiven Modalität des Prädikats: Gerund und Prädikat können unabhängig voneinander negiert werden. Koordinierung erscheint bei der vorliegenden Distribution nicht plausibel als Paraphrase/tbersetzung, sondern allenfalls bei Permutation an den Satzanfang, soweit nicht Blockierung der Koordinierung infolge Divergenz der Modalität von Gerund und Prädikat vorliegt (s.o.). Wenn Koordinierung im 
Einzelfall nicht auszuschließen ist, ist das kein Argument gegen den hier zugrundegelegten strukturtyp, sondern für die Ambiguität der Postposition.

Vgl.:

Ja videl, kak on ulybalsja, pisav éti slova (Paffen II,S.364)

Im Sinne der von Bartsch $72^{77}$ verwendeten Paraphrasen: hier liegt durch während $z u$ explizierende relationale struktur zugrunde im Unterschied zu modaler Struktur, die zu explizieren ist durch wobei und nicht anzuwenden wäre auf ein negiertes Prädikat.

D. h.:

Ich sah, wie er lächelte, während er diese Worte schrieb nicht aber:

Ich sah, wie er lächelte, wobei er diese Worte schrieb oder:

Ich sah, wie er lächelte und diese Worte schrieb

Auch bei der Postposition von russischen pf. Gerundien bietet sich U.E., bei aller Notwendigkeit weiterer Untersuchung der komplizierten Situation, die Möglichkeit, einige wenige semantische strukturtypen $z u$ unterscheiden. Anschaulich wird hier zugleich nochmals die Unmöglichkeit einer systematischen Begründung von strukturtypen aufgrund der Verhältnisse des außersprachlich Bezeichneten.

Trotz Postposition ist mit der Möglichkeit der Vorzeitigkeit zu rechnen. ${ }^{78}$ Bei den folgenden Beispielen wird wiederum Bezug auf das Satzganze angenommen.

Tanja Sorokina ubelala, poobescav prijti posle koncerta

(Adamec II 75, S.175)

Net, on pravil'no postupil, nicegone skazavej= Net, on pravil'no postupil, tro nicegone skazal ej (Ananic 72,5.61) on ich uspokoil, skazav, tto bral knigu (Paffen II,S.371)

$77 \mathrm{Vgl}$. Bartsch 72, S. $173 \mathrm{ff}$

$78 \mathrm{Vgl}$. Mulisch 75, S. 200: "Die Wiedergabe der Vorzeitigkeit der Nebenhandlung gegenüber der Haupthandlung ist bei perfektiven Adverbialpartizipien, auch wenn sie der perfektiven Haupthandlung im Satz nachgestellt sind, häufig und kann daher nicht als Ausnahme betrachtet werden." 
Ja (ne) byl prav, skazav oren' davno, cto...

Für diese pf. postpositiven Gerundien nehmen wir Unabhängigkeit von der Negation des Prädikats an, Unmöglichkeit der Koordinierung und grundsätzlich die Möglichkeit der Permutation an den Satzanfang. So auch in:

On ubilsja, upav s losadi

(Babby 75, S.21)

Vgl. demgegenüber mit Unmöglichkeit der Permutation sowie der Negation des Prädikats alleine, aber mit der Möglichkeit der Koordinierung:

(On) upal, udarivis' golovoj o stupen'ki lestnicy

(Brave $40,5.26$ )

Umschreibungen wie "modale Bestimmung". "Angabe der Art und Weise" u.ä. verwischen derartige strukturelle Unterschiede. Indiz für die Notwendigkeit der funktionalen Differenzierung von Partizipial- und Gerundialstrukturen sind die folgenden Beispiele, in denen für die isolierte Wendung in Anfangsstellung Satzbezug unterstellt wird, für das isolierte Gerund in Endstellung Bezug auf dus Prädikat.

Ne vstredaja socuvstrija so storony Grigorija, Prochor nadolgo umolkal, inogda po celym easamechal, ne poroniv ni slova, surovo nachochlivis' (Rudnev 63, S.178)

Napevnye, zvuenye ..., oni upivalis' sami soboj..., nimalo ne zabotjas' obo mne

(Rứzicka 66,S.50)

Anders als Rúzicka 66, S. 54 ff nahelegt, besteht die grundsätzliche Unterscheidbarkeit der beiden hier illustrierten Typen auch dann, wenn sic nicht gemeinsam in einem Satz auftreten, wenn auch Ambiguitäten nicht auszuschließen sind und natürlich bei gemeinsamem Auftreten im Satz die Unterscheidung anschaulicher ist, so daß Fälle denkbar sind, in denen Disambiguierung der Postposition möglich ist aufgrund der Besetzung der (eindeutigen) isolierten Position am Satzanfang.

Bei den folgenden (ipf.) Gerundien wird Bezug auf das Prädikat angenommen. Das Gerund setzt die Gïltigkelt des Prädikats voraus, das Prädikat alleine kann nicht negiert werden. Möglich scheint hier Paraphrase durch Koordinierung. Möglich wäre auch Negation des Gerund alleine oder des gesamten Prädikatskomple- 
xes. Den unterschiedlichen semantischen strukturtypen entspricht auch, ohne daß hierzu exakte Aussagen möglich sind, das Gesamtbild möglicher semantischer Varianten bei Ubersetzung durch adverbiale Hypotaxe; waren bei dem oben angeführten Typ am ehesten die Konjunktionen während, weil, als, dadurch, daß u.a. angebracht, so entsprechen jetzt eher indem, wobei. Derart $z$ explizierende strukturen sollen wieder unter dem Hilfsbegriff "eine Handlung mit ihrem Merkmal" zusammengefaßt werden.

In der überwiegenden zahl der Fälle handelt es sich um nicht weiter zu spezifizierende Gleichzeitigkeit. Außersprachlich sind die Handlungen von Gerund und Prädikat zwar zu unterscheiden, sie werden jedoch im Satz verwendet zur Darstellung eines Vorgangs, wobei das Gerund eine zusätzliche (und fakultative) Spezifizierung vor dem Hintergrund der Prädikatshandlung gibt. Vgl.:

On sel, razmachivaja rukami = On sel i (on) razmachival rukami (svetlik 70,S.270)

Ona smotrela na menja, veselo ulybajas'= Ona smotrelana menja i veselo ulybalas' (Svetlik 70,5.266)

I Kristall istezaet, ostavljaja poslesebja edkie pary = Kristall istezaet, pricem ostavljaet posle sebjaedkie pary

(Adamec II 75, S.177)

On podosel knej, ulybajas'i popravljaja ocki (Ruanev $63,5.180)^{79}$

Die folgenden ipf. Gerundien drücken das Ziel, den Zweck der Haupthandlung aus. 80 Auch hier ist Negation des Prädikats alleiné nicht möglich.

Ljudi razbezalis', spasajazizn' (Adamec II 75,S.176) My ostanovilis', podzidaja prochozego, u kotorogo mozno bylo

79 Rudnev a.a.O. sieht in solchen Fällen das Gerund als obligat und jede Paraphrase als unmöglich an wegen völligen Zusammenfalls der Handlungen

$80 \mathrm{Vgl}$. Mulisch 75,S. 202: "Die konativ-finale Bedeutung der durch das Adverbialpartizip ausgedrückten Handlung bleibt auch dann bestehen, wenn man den Satz -deutsch und russischso umformt, daß er zwei koordinierte Prädikate (eine Prädikatreihe) enthält... Die Bedeutung der Gleichzeitigkeit ... 
sprosit' dorogu = My ostanovilis', ktoby podokdat' prochozego, u kotorogo... (Mul isch $75, \mathrm{~S} .208$ )

Auch bei cen folgenden pf. Gerundien legen wir die Klassifizierung "eine Handlung mit ihrem Merkmal" zugrunde. Das pf. Gerund hat resultative Bedeutung.

Für derartige Belege ist die Postposition relevant zum Ausschluß der Vorzeitigkeit. ${ }^{81}$ Das Ergebnis der Gerundialhandlung wird relevant für die Gegenwart der Prädikatshandlung: nach ipf. Prädikat: 82

Źen'ka sidel, podnjav koleni $k$ podborodku = Żen'ka sidel s kolenjami, podnjatymi k podborodku (Adamec II 75.S.179) Devocka slusala ego, zataiv dychanie (Paffen III.S.209) Zur Relevanz der Postposition vgl.: On tital tekst, ob-jasniv ego soderzanie (Paffen II,S.370) d.h.: er las den Text abschnittweise und erklärte jeweils den gelesenen Abschnitt

Vgl. demgegenüber Voranstellung, die nicht wechselweise Abfolge, sondern Vorzeitigkeit signalisiert.

Vgl. auch:

Orator govoril, vysoko podnjav golovu : vysoko podnjav golovu, orator zagovoril

(Brave 40,S.25)

Bei Anfangsstellung liegt vor "zwei voneinander verschiedene Handlungen" mit Satzbezug und Möglichkeit der Negation des Prädikats alleine.

Ebenfalls als"eine Handlung mit ihrem Merkmal" (Bezug des Gerund auf das Prädikat) wird das pf. Gerund nach pf. Prädikat in den folgenden Beispielen mit nichtvorzeitiger (resultativer oder gleichzeitiger) Bedeutung klassifiziert: ${ }^{83}$

80 ... tritt in diesem Falle auf Grund der Handlungsidentität und der konativen Bedeutung der zweiten Handlung in den Hintergrund."

$81 \mathrm{Vgl}$. Rủzicka $66, \mathrm{~S} .47 \mathrm{ff}$

82 Vgl. Kízková 69, Synt.ch.. S. 121ff; Paffen II.S. 370ff; Brave 40,S.25ff; Mulisch 75,S.199ff;Vinogradov 47,S.388ff

$83 \mathrm{Vgl}$. Mulisch 75,S.199: "Dem pf. Aspekt der finiten Verbalform und des Adverbialpartizips ist keinerlei Information über die zeitliche Reihenfolge der beiden Handlungen zu entnehmen.": Brave 40.S.26: Vinogradov 47.S.390fe 
On vyšl iz komnaty, ne skazav ni slova (Leitfaden,S.149)

v 1582 g. Papa rimskij Grigorij XIII provel reformu kalendarja, propustiv 10 dnej ... (Leitfaden,S.150)

Die Relevanz der Postposition für den AusschluB der Vorzeitigkeit wird anschaulich $2 . B$. in:

On spisal tekst, podkerknuv v nem suscestritel'nye

(Brave 40,S.26)

On prozital knigu, vypisav iz nee nutnye mesta

(Brave 40,S.26)

Odnako Sarl' predpocel udrat' iz doma, pustivsis' vdogonku za cirkovoj naezdnicej

(Rủzicka 66,s.45)

Rúzicka klassifiziert diesen Beleg als "Prädsec" und betont die hier vorliegende Tempusrelation "non ante". Gerade wenn dies zutrifft und aufgrund der oben aufgefuhrten Kriterien (auch Ruzicka selbst nennt ja die Geltung der Negation des Prädikats auch für das Gerund als Kriterium für "Advb") scheint uns diese Klassifizierung nicht zutreffend. Dem entspricht auch die von Rúzicka selbst betonte Unmöglichkeit der Permutation an den Satzanfang. 84

Grundsätzlich ist daran zu erinnern, daß der Form des pf. Aspekts selbst keine allgemeinguiltige Information über die Zeitenfolge entnommen werden kann. Dies wird bei postpositivem Gebrauch der pf. Gerundien, wie er im Russischen häufig ist, besonders deutlich; während Präposition zum Ausdruck der Vorzeitigkeit tendiert, ist bei stellung nach pf. präteritalem Prädikat auch mit Nachzeitigkeit zu rechnen:

Pri nasem priblizenii tolpa rasstupilas' na obe storony, obrazovav rod irokoj dorozki= Tolpa rasstupilas'na obe storony i obrazovala rod sirokoj dorozki (Brave 40,S.25-26)

zamaza otpala, ogoliv stenu = 2amaza otpala i ogolila

$84 \mathrm{Vgl}$, auch Rủicka,sS, S. 181 (zu aksl. postpos. prät.. meist nichtipf. Partizip in der Kurzform): "Postposition ist die bevorzugte und angemessene Stellung bei Einheit der Handlungen. Die Konstruktion setzt sich in der literatursprachlichen Tradition des Russischen geradlinig bis in die moderne sprache fort. Die Fügung/perfektives Verb-perfektives Gerundium/ bei Einheit der beiden Handlungen ist in der modernen Sprache der Publizistik und auch anderer literarischer Bereiche lebendig." 
stenu

(Brave 40,S.26)

Masina ostanovilas', pregradiv put' transportu = Masina ostanovilas', tak to pregradila put' transportu= Das Auto hielt an, so daß es die Straße versperrte (Mulisch 75,S.208) Hier liegt Nachzeitigkeit im Sinne einer nicht weiter spezifizierten Reihenfolge oder mit zusätzlicher konsekutiver Nuance vor. Das Gerund setzt die Gültigkeit der Prädikatshandlung voraus. Permutation an den Satzanfang ist nicht möglich bzw. würde $z u$ anderem strukturtyp führen. Auch wenn im Sinne des auBersprachlich Bezeichneten meist auf die semantische Eigenständigkeit der Gerundialhandlung verwiesen wird, verhalten sich derartige Belege auf der Ebene der kommunikativ-semantischen Gliederung analog zum Typ "eine Handlung mit ihrem Merkmal". d.h.. es wird Bezug auf das Prädikat und nicht auf das Satzganze angenommen. Dem entspricht die Möglichkeit der Paraphrase durch koordiniertes finitverbales Prädikat, 85 die bei Postposition und Satzbezug nicht beobachtet wurde (s.o.), sowie die Betroffenheit durch die Negation des Prädikats. Schematisch zusammenfassend ergibt sich für russ. $N+V+I+G e r$ folgendes Bild:

Der umgangssprachlich als "zwei voneinander verschiedene Handlungen" umschriebene Typ der Konnexion ist gekennzeichnet durch Bezug auf das Satzganze: 86

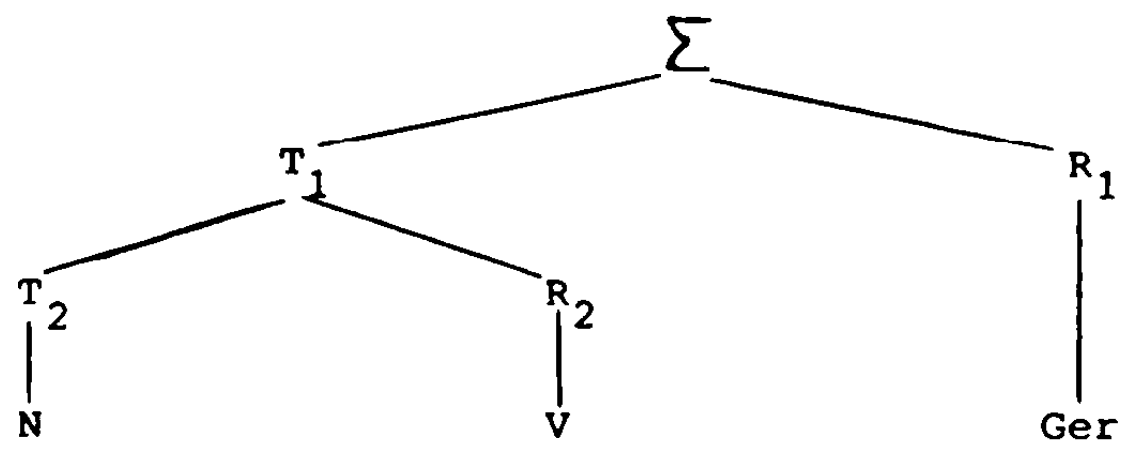

$85 \mathrm{Vgl}$. hierzu Ktizková 69, Synt.ch., S. 122ff, die für derartige Strukturen als Ausgangspunkt der Ableitung nicht wie sonst nach dem Subjekt eingebetteten Satz annimmt, sondern eine dem Prädikat nachgestellte finitverbale Form in parataktischer Anordnung.

86 Vgl. z.B. AG 70, S. 630 zum Satzdeterminant in Endstellung: Adamec 66, P.s., S. $35 \mathrm{ff}$ 
In Analogie zu Anfangsstellung und Thematisierung der isolierten Wendung steht das Gerund außerhalb der Reichweite der Negation des Prädikats. Weiss faßt beide Fälle des Satzbezuges zusammen als "Kulisse" gegenüber dem Satz. Nicht möglich ist (im Gegensatz zur Anfangsstellung) bei diesem Typ Koordinierung mit der satzschließenden Prädikation als Paraphrase/tbersetzung, jedenfalls bei Nichtinversion von $\mathrm{N}$ und $\mathrm{V}$.

In der Ubersetzung entsprechen diesem strukturtyp adverbiale Nebensätze mit z.B. während, weil, wenn, nachdem u.a.. nicht jedoch mit wobei. 87

Das Gerund steht außerhalb der Reichweite der nichtassertiven Modalität des Prädikats.

Vgl. z.B. poln.:

Wpadnij do mnie, wracajoc uczelni! (Weiss 77,5.304)

Entgegen z.B. Kłíkková, żaža 64, Kacala 71 u.a., die undifferenziert sämtliche isolierten Strukturen als "Prädsec". "freien doplnok" u.ä. beschreiben, ist die Ambiguität der Postposition mit Isolierung festzuhalten. Der umgangssprachlich als "eine Handlung mit ihrem Merkmal" umschriebene Typ wird im Schema nicht als dem Prädikat hierarchisch übergeordnet dargestellt, sondern auf gleicher Stufe; ob sich diese beiden Typen in der Intonation unterscheiden, kann hier nicht beurteilt werden. Vgl.:

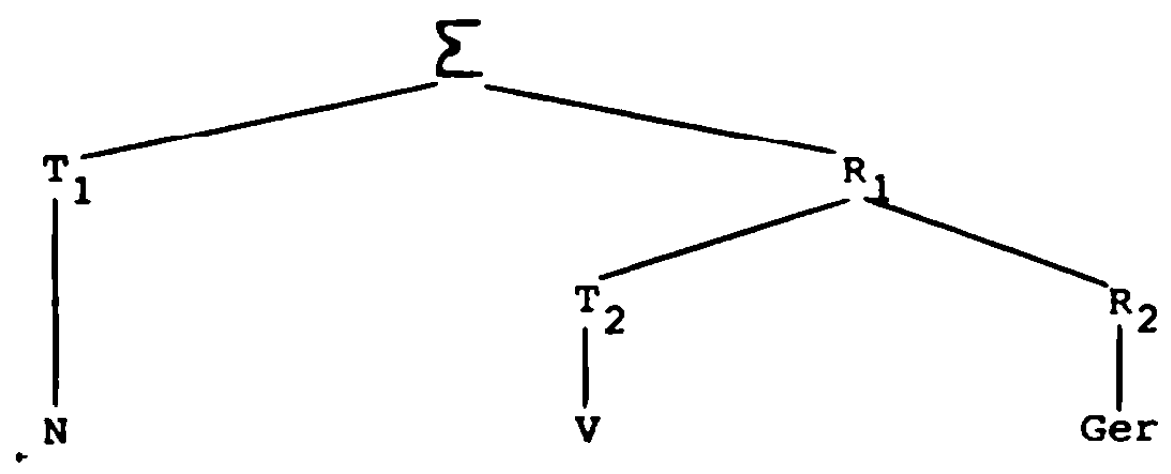

Wenn das Gerund steht, darf nicht das Prädikat alleine negiert

$87 \mathrm{Vgl}$. hierzu auch die Aufzählung semantischer Varianten für isolierten "PO" bei Kfizková 69,PO (kaus.. temp.. konzess. u.a.): zur statistischen Relevanz der Wortstellung für die Verteilung der Varianten vgl.a.a.o..s. $28 \mathrm{ff}, 32 \mathrm{ff}$ 
werden, sondern allenfalls das prädikat mitsamt dem Gerund. Möglich ist in Postposition bei diesem Typ Paraphrase durch Koordinierung. Die nichtassertive Modalität des Prädikats erstreckt sich in solchen Fällen auch auf das Gerund, hierzu ist also Fokussierung des Gerund nicht Bedingung. 88

Vgl. hierzu nochmals (s.o.) aksl.:

sodnse pokažite se iereomn

Soweit sich überhaupt Ubersetzung durch konjunktionale Hypotaxe anbietet, kommt bei diesem Typ u.E. wobei, nicht während in Frage.

8.3.2. Russ. $V+I+G e r$

Grundsätzlich analog $z u \quad N+V+I+G e r$ bewerten wir den Distributionstyp $\mathrm{V}+\mathrm{I}+\mathrm{Ger}$.

Hatten wir bei $\mathrm{N}+\mathrm{V}+\mathrm{I}+\mathrm{Ger}$ auch für ipf. Gerundien den Typ "zwei voneinander verschieden Handlungen" vorgesehen, so findet sich allerdings jetzt in unserem Material bei $\mathrm{V}+\mathrm{I}+\mathrm{Ger}$ nur der Typ "eine Handlung mit ihrem Merkmal". auch wenn Gegenbeispiele vermutlich nicht auszuschließen sind.

Vgl.:

Śl, postukivaja palkom

(AG $70,5.645)$

Krepilas', ne pokazyvaja bespokojstva

(AG 70,S.645)

Vgl. auch mit Gerund in Abhängigkeit von nichtfinitverbalen Formen:

(Nam) prijatno byloguljat' po lesu, sobirajagriby (Mulisch 75.S.206)

Vrac predpisal bol'nomu lezat', ne vstuaja s posteli

(Babby, S. 22)

Ja sledoval za starikom, sedsim, Cut' prichramyaja, vperedi

(AG $70,5.645$ )

vrac razresil me projti k bol'nomu, sobljudaja tisinu

(Mulisch 75.5.207)

$88 \mathrm{Vgl}$. demgegenüber Weiss 77, S. 301 zum Poln.:"Nur bei Fokussierung kann die Part-II-Konstruktion u.U. auch eine nicht assertive Modalität (Frage, Aufforderung, Wunsch etc.) zum Ausdruck bringen." 
Als Gegenbeispiel zur oben angefihrten Tendenz vgl. allenfalls mit $N+\ldots+V+I+G e r$ :

Kak chudoznik spolna otdaetsja delu, daze esli v glubine dusi $v$ nego ne verit, i stremitsja blestnut' $v$ masterstve, soznarja, cto ono uze otzivaet svoj vek... (F,S.186)

Beide strukturtypen finden sich bei pf. Gerund. Dabei fassen wir im Sinne unserer Klassifizierung sowohl resultative Bedeutung wie Bedeutung der zeitlichen Reihenfolge als "eine Handlung mit ihrem Merkmal"zusammen (s.o.).

Vgl. z.B.:

Vysla iz komnaty, gordo podnjav golovu

Ulozila rebenka spat', ukryvego odejalom (AG 70,5.645)

spit, raskinuvsis'

(AG 70,S.645)

Vgl. mit Gijligkeit der Aufforderung auch für das Gerund (bei Nichtfokussierung):

Povtorite predlozenie, zameniv odnosloznoe "glub" trechsloznym "glubina" = Wiederholen sie den satz und ersetzen sie dabei das einsilbige "glub" durch das dreisilbige "glubina"

Povtorim predlozenie, zameniv... $\quad$ (Paffen II,S.371)

Rasskazite anekdot, postaviv vmesto "kupec" podlezascee "ja"

(Paffen II,S.374)

Demgegenüber ist Koordinierung bei den folgenden Belegen nicht möglich bzw. wïrde auf einen anderen strukturtyp verweisen: usel, poruvstuovav sebja lisnim (AG 70.5.645) Unpersönliche Konstruktion:

$\checkmark$ tu minutu esce mozno bylo dognat' Natasu, schvativ taksi

(Adamec 75, S.172)

v étom moźno ubedit'sja, prodelav prostojopyt

(Sint.r.j.74, S.137)

Schematisch ließe sich, in Analogie zu den oben aufgefuhrten Strukturen in Präposition, folgende Unterscheidung vorschlagen: 


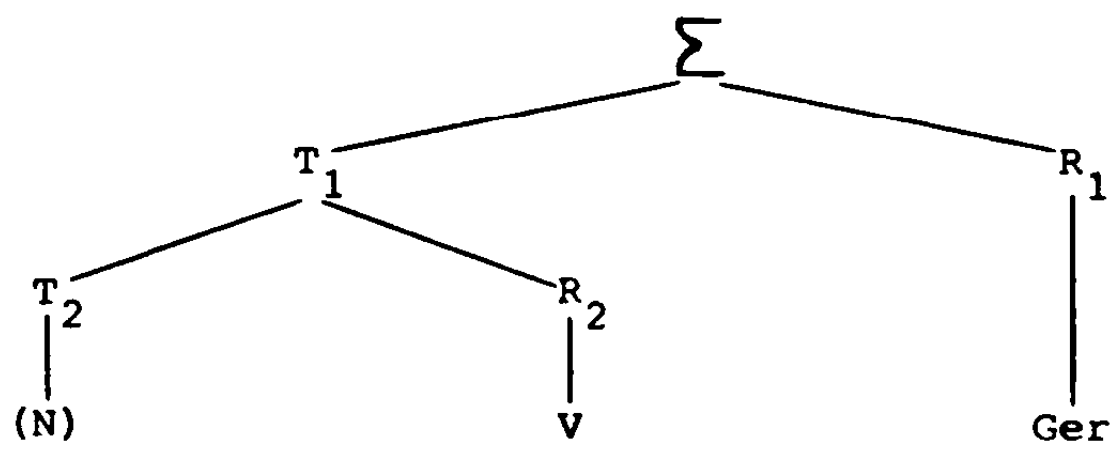

gegenuiber:

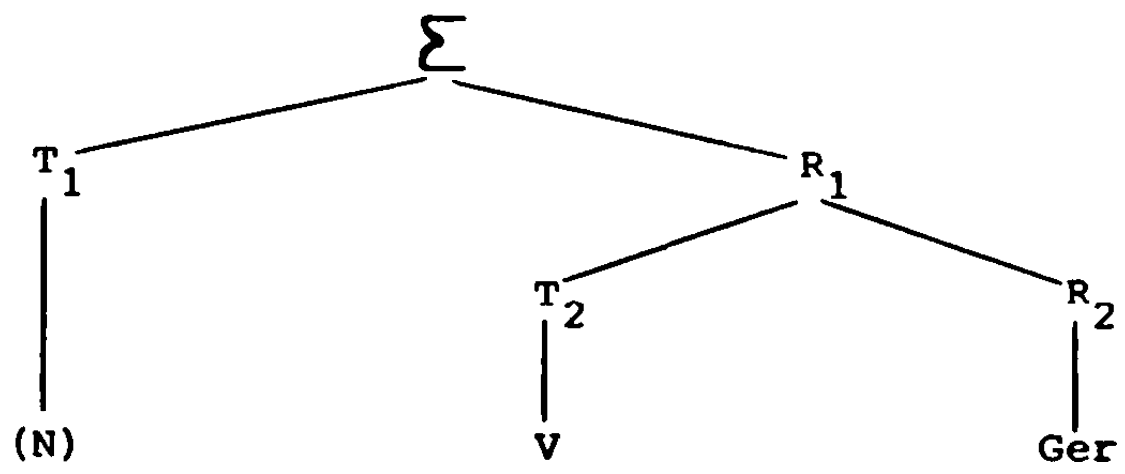

8.3.3. Russ. $V+I+G e r+I+N$

Neben der "normalen" Distribution $(\mathrm{N}+) \mathrm{V}+\mathrm{I}+\mathrm{Ger}$ tritt auch auf $\mathrm{V}+\mathrm{N}+\mathrm{I}+\mathrm{Ger}$ (vergleichsweise selten) sowie, mit zwei Beispielen in unserem Material, $\mathrm{V}+\mathrm{I}+\mathrm{Ger}+\mathrm{I}+\mathrm{N}$.

Diese Distributionen zeigen mit besonderer Anschaulichkeit die Notwendigkeit, Wortstellungstypen des Satzes im Hinblick auf ihren textuellen zusammenhang $z u$ erörtern. Eine Klassifizierung innerhalb des Satzes stößt auf Schwierigkeiten. $\mathrm{Vgl}$. mit $\mathrm{V}+\mathrm{I}+\mathrm{Ger}+\mathrm{I}+\mathrm{N}$ :

Na zaborach balagurjat, podprygivaja, vorob'i (Gnevko 75,5.85) -Kngda poezd priedet?- sprosil, vojdja na platformu, rabocijs sundukom i cajnikom cerez plečo

(Morison, S.41)

U.E. ist am einfachsten in beiden Fällen Bezug nur auf das Prädikat anzunehmen und im Sinne unseres Schematismus darzustellen: 


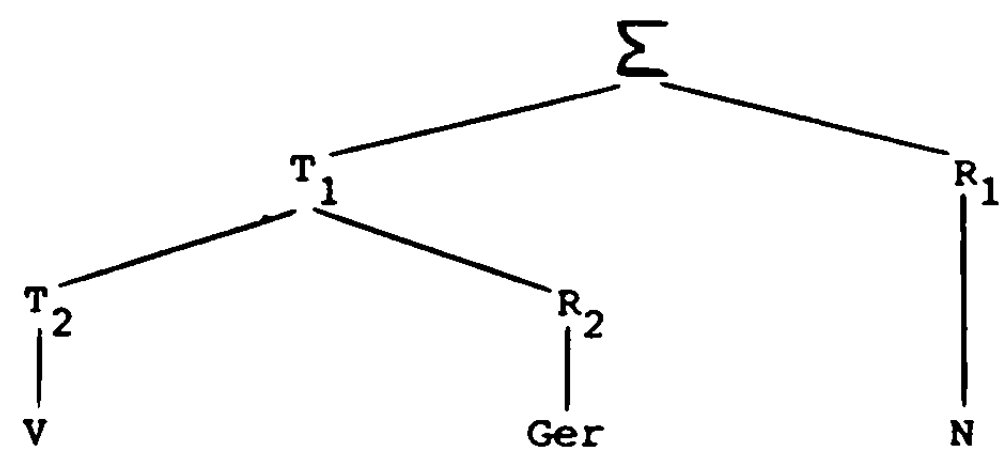

8.3.4. Russ. $V+N+I+G e r$

Bei Endstellung des isolierten Gerund und Inversion von $\mathbf{N}$ und $\mathrm{V}$ ist eine klare Entscheidung über den funktionalen status erschwert und es stellt sich die Frage, ob hier nicht eher statt von Ambiguität hinsichtlich Satzbezug : Bezug auf das Prädikat von Neutralisierung dieses semantischen Gegensatzes zu sprechen ist. Nicht nur Ambiguität, sondern Neutralisierung liegt für die Position "isolierte Endstellung bei Inversion von N und V" möglicherweise auch vor hinsichtlich der zuordnung zum subjekts- oder Prädikatskomplex: es finden sich hier auch Partizipien in der Stellung nach V, für die außer Relativsatzparaphrase (s.o.) adverbialer Nebensatz infrage kommt, und es wird für Gerundien in dieser Position u.a. auch Relativsatzparaphrase angefürt. Kennzeichnend für den hinsichtlich dieser syntaktisch-semantischen Gegensätze potentiell neutralen Charakter dieser Distribution düfte sein, daß für Partizipien und Gerundien neben den üblicherweise zitierten Paraphrasen und Ubersetzungen gerade hier auch selbständiger nachgestellter Satz nicht auszuschließen ist.

Vgl. z.B.:

Na vetke ivy sidela malen'kaja seraja ptica, izdavaja prijatnyj tresk = ...i izdavala prijatnyj tresk (sint.r.j.74,s.138) Stupal on tjazelo, na kazdom rotlivoe telo $=\ldots i$ na kazdom sagu perekidyval ...

(Morison, S.30)

Vot vystupat orator, gorjaco dokazyvaja cto-to, ego re ' $^{\prime}$ napolnena zivym Celovereskim Cuvstrom = Da trittein Redner auf, der 
mit Eifer etwas beweist, ...

(Paffen II,S.374)

Koe-gde popadaetsja molodaja berezka s melkimi, klejkimi list'jami, brosaja dlinnuju, nepodviznuju ten' na glinistye kolei i vesennuju travu = Stellenweise trifft man auf eine junge Birke mit ihren kleinen klebrigen Blätern, die einen langen, reglosen Schatten auf die lehmigen Fahrinnen und das aufspieBende (Frühlings-) Gras wirft.

(Paffen III,S.211)

I na nej igrala ulybka, prijatno socetajas' s cut' robkim...

$v 2 g l j a d o m$

(F,S.35)

... zametil Nikolaj Parfenovic, neskol'ko skonfuzjas'

(Morison, S.43)

-A nebesnuju sinevu ty toze nazoves' obmanom?- vozrazil muz,

vzgljanuv v ee storonu

(F,S.37)

Gde-to blizko udaril grom, napugav vsech (Sint.r.j. 74,S.137)

Čirknula spicka, na sekundu osvetiv licostarika=

(cech.): Škrtla zápalka a na okamzik osvetila starcúv oblikej

(Sint.r.j. 74,S.138)

Naturlich böte sich als einfachste schematische Form für derartige Beispiele an:

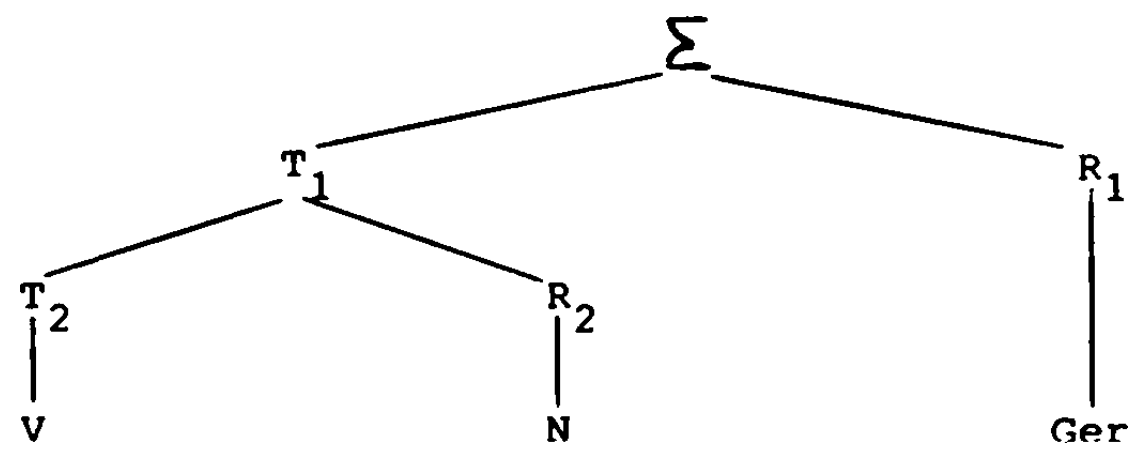

Im Einzelfall ist jedoch auch Bezug nicht auf das Satzganze, sondern nur auf das Prädikat nicht auszuschließen.

8.3.5. Russ. $\mathrm{V}+\mathrm{N}+\mathrm{I}+\mathrm{P}$

Dieser Distributionstyp war schon in Zusammenhang mit Partizipialkonstruktionen, die hinreichend im Rahmen der Nominalphrase beschreibbar sind, angesprochen worden. Es sei daran erinnert, daß 
in dem Material deutscher Partizipialkonstruktionen bei Bungarten nur in dieser Distribution Ambiguität hinsichtlich der Zuordnung zum Subjekts- oder Prädikatskomplex auftritt (Nichtrestriktivität dabei vorausgesetzt). 89 Hier und auch für das Russische ist zu fragen (s.o.), ob nicht der Gegensatz Zuordnung zum Subjektskomplex : zum Prädikatskomplex weitgehend neutralisiert ist.

Vgl. mit pass. Partizip:

Po doroge sla avtomašina, nagružennaja otbornym zernom pšenicy - Po doroge la avtomasina; ona byla nagruzena otbornymzernom psenicy

(Ananic 72, S.33)

Vgl.:

$2 a$ stolom sidel student, razmysljajuß́tij (razmyšljaja) nad zadaniem (Georgieva 68, S. 104)

Vperedi nas šla żenšcina, prjatavsaja licov vorotnik suby - Vperedi nas la zenšina, prjaca lico v vorotnik suby

(Adamec II 75, S.186)

Razgovor otcas gostem prervalamat', pozvaviaja ich uzinat'= ... prervala mat', tak kak ona pozvala ich uzinat'

(Adamec II 75,S.187)

... muzykanta, s kotorym stol' besposcadno obołlas' sud'ba, vysoko ego voznessaja i zatem nizrinuvsaja v bezdnu ...

(F,S.25)

Pozdno prichodit ona domoj, ustalaja i ozjabłaja

(Rudnev 63,S.164)

Die oben angeführten Belege für $\mathrm{V}+\mathrm{N}+\mathrm{I}+\mathrm{P}$ ließen sich eventuell als Gegenbeispiele zu der bei Rúzicka angedeuteten Unmöglichkeit von Partizipien als "sekPräd" in Postposition anführen, 90 so daß auch in Stellung nach dem Prädikat Gerund durch Partizip ersetzt werden kann. Weitestgehend ausgeschlossen ist der Gebrauch von Partizip als "sekPräd" in Postposition, solange nicht Inversion von $\mathrm{N}$ und $\mathrm{V}$ vorliegt. In unserem Material treten in dieser Position keine akt. Partizipien auf, sondern z.B. Adjektive und pass.

89 Zum Deutschen vgl. Sviblova 62: vgl. auch Ražicka, SS, S. 161f1 zu $V+N+P_{k}$ und a.a.O.. S. $232 f f$

$90 \mathrm{Vgl}$. ders. 66, S. $49 \mathrm{ff}$ 
Partizipien.

Vgl. allenfalls, mit Adjektiv koordiniert:

Deti vozvrastalis's katka tol'ko vecerom, razrumjanivisesja veselye

(zaźa $65,5.67)$

8.3.6. Skr. postpositives Gerund

Hier werden Belege mit und ohne explizites Subjekt und, aufgrund der geringen Relevanz der Isolierung, Belege mit und ohne Isolierung des postpositiven Gerund angefürt, so daß der Typ der Distribution darzustellen ist als

$$
(N+) V+(I+) \text { Ger. }
$$

Dem Typ "Satzbezug" werden zugeordnet:

Bugarce se spavat' naucilo po planini ovce cuvajuci $=\ldots$ dok je cuvalo... (Musić 35,S.143) 91

Video sam te ulazé̃i = video sam te kad sam ulazio (Pesikan 59, S.95)

Bila je uzbudena, osvrtala se, bojéci se dace starica spaziti da nema novcanika i poceti vikati = Bila je uzbudena, osvrtala se jer se bojala... (Tezak-Babić, S. 249)

Ne smedne mu kazati da je imala tri kćeri pa da ih je nestalo, boječi se da ne bi stojša tumario u svet da ih trazi

(Musić 35,S.145)

Das heißt: wenn auch in Postposition Belege des Typs "eine Handlung mit ihrem Merkmal" uberwiegen, es ist auch im Skr. bei Nachstellung $z u$ rechnen mit Beispielen, für die Negation des Prädikats alleine möglich ist. Nicht möglich ist hier Koordinierung.

Analog mit Inversion:

Skoci domacin znajuci, šta se dogodilo, pak prinese svijecu (Musić 35.S.146)

Jeste li videli kuda je tako naglo nestalo sunce, spustivi se zakrvavljeno iza bregova = ...kuda je tako naglo nestalo sun-

91 In Music's Terminologie handelt es sich um "appositives" Gerund, vgl. a.a.0. S. 143: "Pita se, sto je B. ovce cuvajuci uradilo, a ne, kako se spavati naucilo" 
ce, posto se spustilo zakrvavijeno iza bregova

(Tezak-Babić,s.249)

Kamo ti zena? zapita moja mati zacudivil se vrlovideci me sama (Belić 69,S.196)

Für die weit uberwiegende Zahl der skr. postpositiven Gerundien in unserem Material wird der Typ "Einheit eines Vorgangs" zugrundegelegt aufgrund der Möglichkeit der Koordinierung sowie der Unmöglichkeit der Negation des Prädikats alleine. Als Konjunktion bei der Ubersetzung ins Deutsche durch adverbialen Nebensatz kommt in Frage wobei, indem, nicht aber während (s.0.). On udari drugim putem moleci se bogu da mu da dobru srecu= ... i stane se moliti bogu...

(Belić 69,S.194)

(Mati) ga stane odvraćati i moliti da ne ide, kazujući mukako je to bilo davno (Musić 35,S.146)

starac je stupao pognuto vukući tesko noguza nogom = Starac je stupao pognuto i vukao tełko noguza nogom

(Tezak-Babić,s. 248)

Misleci to, vuk je isao za ovicom, zastajkujuci kad ona zastane i opružajuci korak kad ona ubrza ritamigre = .. vuk je iłao za ovicomi zastajkivao kad ona zastane... (Tełak-Babić,s.248) I la je polakoulicom, zaustavljajuci se pred svakim izlogom (Biricz,S.94) Šćer ga poßl je da spava obećavajuci da cee ona sve to uciniti (Music 35, s.147)

A on se osere na cara govoreci, da je on carev sin

(Musić 35, S. 146)

Soweit bisher in unserem Material uberhaupt präteritales Gerund bei Nichtinversion in Endstellung auftritt, bezeichnet es nach den oben aufgefuhrten Kriterien eine Handlung mit ihrem Merkmal. Voranstellung wuirde $z u$ einer anderen Interpretation fuhren: Ja ovu strasnu muku otrpim ne maknuvii se = ...i ne maknem se

(Belić 69,S.196)

Utini toliko drugi besposlica, da ni sad jos ne mogu na nji pomisliti ne nasmijavsi se (Musić 35,S.148) Pa okrenu od Bijela \$atora zametnuvi koplje naopako

(Musić 35, S.148)

oplinski bilježnik, blagajnik i pisari promrmlaše nešto i ne 
pogledavisi Mike = a da nisu pogledali Mike

(Teźak-Babić,S.149)

Das Gerund nennt ein nichtvorzeitiges Merkmal der Haupthandlung und antwortet auf die Frage: Kako?

Vgl. auch:

Ode ni rijéci ne progovorivsi

(Resetar 22, S.181)

otidemo svaki u svoju sobu spavati ne pogovorivis jedan s drugijem ni jedne rijedi

(Musić 35, S.148)

8.3.7. Aksl. postpositives $\mathrm{P}_{k}$

Die Relevanz des Stellungsgegensatzes vor : nach dem Prädikat für aksl. $P_{k}$ hinsichtlich der statistischen Verteilung der präsentischen/präteritalen $P_{k}$ und der graduellen Reduzierung der peripheren Prädikativität wurde schon erwähnt. Grundsätzlich besteht in Präposition die Tendenz zur Konfrontierung der Handlungen von $P_{k}$ und Prädikat, in Postposition zur "synthetischen" Darstellung als Einheit einer Handlung bis hin zur Annäherung an die Bedeutung einer adverbialen Bestimmung. Ambiguität hinsichtlich der Typen der Konnexion ist dabei eher in Postposition als bei Präposition $z u$ beobachten, während im Russischen auch bei Gerund in stellung vor dem Prädikat (außer bei Ger+I $+N+V)$ mit Ambiguität hinsichtlich Satzbezug : Bezug auf das Prädikat zu rechnen ist; dies ist ein Ergebnis des Ubergangs der alten peripheren Prädikativität zur untergeordneten Prädikativität im Russischen.

Bezeichnend für die reduzierte prädikative selbständigkeit des postpositiven $P_{k}$ im Aksl. ist auch, daB Koordinierung des $P_{k}$ durch die Konjunktion $i$ mit der finitverbalen Präikation ungleich seltener ist als bei Voranstellung des Partizips. 92

Bei aksl. präsentischen $P_{k}$ in stellung nach dem Prädikat ist entgegen der Tendenz der Postposition zu "synthetischer" Darstellung auch mit dem Typ "zwei voneinander verschiedene

92 Vgl. Rủžička, ss, S. 102ff, $187 \mathrm{ff}$ 
Handlungen" $2 u$ rechnen, wenn das Partizip eine sinnliche oder psychische Wahrnehmung als Voraussetzung. Grund u.ä. für die Haupthandlung bezeichnet. 93 Ublicher ist Stellung vor dem Prädikat. Die Nachstellung kann bedingt sein durch stilistische Gründe, Kontext oder Inversion von Subjekt und Prädikat. mbnozi vêrovaş vo ime ego. videste znamenok ego. eze tvoréale J 2,23 (SS,S.157)

is rele imv. blodite ne veldqute krnigb Mt 22,29 (SS,S.158) ne sego li radi blodite. ne vedgste kbnigb Mr 12,24 (SS,S.158) Wieder ergibt sich die Möglichkeit der Negation des Prädikats, ohne daß $P_{k}$ betroffen wäre.

Beide Typen sind denkbar bei dem ambigen

cototb me uceste (SS,S.160)

In: ne crotob me urgste

gilt die Negation entweder nur für das Prädikat oder für Prädikat und Partizip; d.h.:

Sie erweisen mir keine Ehre, wenn sie lehren, daß... oder: sie ehren mich nicht dadurch, daß sie lehren (sondern auf eine andere Weise)

Vgl. mit Gültigkeit der Negation des Prädikats auch für das Partizip:

farisei bo. i vsi ijudei. aste ne umyvajotb roku torgste. ne édetr. Mr 7,3 (SS, S. 149)

Das Partizip begründet die "Fragwürdigkeit" der Prädikatshandlung und liegt selbst nicht im Bereich der Frage: kako vy mozete verovati. slavo drugn. ot druga priemljoste... J 5.44 (SS,S.160)

Bei derartigen Belegen zeichnet sich im übrigen die Schwierigkeit ab, zu entscheiden, ob peripherprädikatives Partizip des Typs "zwei Handlungen" oder eher attributive Determination (Relativsatzparaphrase) vorliegt: vgl.:

Néstr bo drévo dobro trore ploda zola. ni drévo zblo tvore ploda dobra L 6.43 (SS,S.161) 94

$93 \mathrm{Vgl}$. Rửickka, SS, S. $157 \mathrm{ff}$

$94 \mathrm{Vgl}$. a.a.0.. S. $161 \mathrm{ff}$ zu negierten Existenzsätzen und S. 354 
Zugrunde liegt die zumindest stark reduzierte Prädikativität des merkmallosen Gliedes der aksl. Korrelation.

Auch in den folgenden Belegen aus MS nennt das postpositive präs. Partizip 95 die Voraussetzung, Motivierung usw. für die finite Handlung, nicht einen immanenten Aspekt. Koordination erscheint nicht plausibel, sie wäre allenfalls möglich bei Permutation des Partizips vor das finite Prädikat, wie sie auch im Material bei lexikalischer Analogie belegt ist. Als Ubersetzung kommt am ehesten in Frage konjunktionale Hypotaxe (z.B. mit. weil, während).

i idocho vorsrobnskuju zemlu, zelaje i chote ukrépiti préstoln roditels moichs (MS, S.187)

Ja gosopodinn Balsa po milostibozie stvorichn milosto gradu Dubrovniku ... spomenujuke staro prijatelnstuo i ljuboun (MS,S.193)

Vgl. mit Negation des Prädikats, nicht aber des Partizips: kto troi vragn pribégne $u$... da ti ne ispakosti nistare, u nas.. bude (MS, S. 22)

i ako kto pribegne ōtovass u zemlu kralevostru mi, da ne isopakosti vamn nicto, stoe u zemlu kralevnstva mi (MS,S.46)

Für die folgenden präsentischen $P_{k}$ in Postposition wird wieder der Typ "eine Handlung mit ihrem Merkmal" zugrundegelegt mit deutlicher Tendenz zur Unterordnung des $P_{k}$ unter das Prädikat. $P_{k}$ hat wieder zur Voraussetzung die Gültigkeit der Prädikatshandlung, diese kann nicht alleine negiert werden. Die Neigung zur Unterordnung des $P_{k}$ unter die satzschließende Prädikation ist insbesondere dann ausgeprägt, wenn dem Partizip die "Stütze" durch ein subjektsnomen fehlt. 96

Bei den folgenden Beispielen nennt das Partizip Absicht, Zweck usw. der Haupthandlung. Permutation ist nicht möglich bzw. verändert die struktur. 97

i pristopi kr nemu diẻvolr. iskušaje i i rełe Mt 4,3 (SS,S.140)

$95 \mathrm{Vgl}$. M.. S. $24 \mathrm{ff}$

96 Vgl. Rüzicka, SS, S. 348

$97 \mathrm{Vgl}$.a.a.o.. S. $139 \mathrm{ff}$ 
i pride kz nemu prokaženr mole i. i na kolénu padaje i gle emu Mr 1,40 (SS,S.140)

i obr chozdaase gradoce uç Mr 6,6 (SS,S.143)

Vgl. als Endstadium der Verdichtung im Sinne einer "synthetischen" Darstellung:

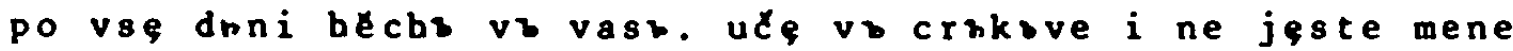
Mr 14,49 (SS,S.141)

Vgl. $P_{k}$ zur Angabe von Begleitumständen, Art und Weise der Haupthandlung ${ }^{98}$, im Einzelfall mit möglicher Affinität zu Adverbialbestimmung und Möglichkeit der Permutation vor V:

... pridose na grobr. nesoste eze ugotovase aromaty

L 24,1 (SS, S. 145)

i pride kr nimb po morju chode

Mr 6,48 (SS, S. 146) 99

Partizip und Prädikat bezeichnen eine Handlung unter verschiedenen Aspekten: 100

Shziraachq ze se mezdju sobojq urenici. ne domysleste o komb gletw J 13,22 (SS,S.152)

i glaase. b̧gsvte ba

L 1,64 (SS,S.153)

Vgl. auch die Belege aus MS zum postpos. präs. Partizip: ${ }^{101}$ temnze i azr, rabo tvoi Stefann Ourosn, ... pripadaju ko pré cistima nogama troima, milosti prose i prostenije grêchomn moimn (MS, S.67) sego radi i azn pripadaju védyi troje clovêkoljubye, molju se vnpie sice ti Isou Christe... (MS,S.9) 102 i tagda prêde prédn kralevustvo vironi nasb zupann vlnkomirn Semikovićn, skazue namn, kako ... (MS,S.226)

Daß gerade beim Typ "eine Handlung mit ihrem Merkmal" als Para-

$98 \mathrm{Vgl}$.a.a.0.. S. $145 \mathrm{ff}$

99 Vgl. hierzu a.a.0.. S. 145:"Das gleiche Partizip kann bei schwacher Semantik des Hauptverbs die Hauptlast der Prädikation tragen."

$100 \mathrm{Vgl}$. a.a.O.. S. $152 \mathrm{ff}$

$101 \mathrm{Vgl}$. M.. S. $20 \mathrm{ff}$

$102 \mathrm{Zu}$ solchen formelhaften Wendungen vgl. auch Rúzicka,SS, S. $163 \mathrm{ff}$ 
phrase und als Ubersetzung Koordinierung zweier finiter Prädikate plausibel ist $(\mathrm{s.0.)}$, zeigen Beispiele aus MS wie: K tebe ...pripadaju i molju se (MS,S.109) 103

Von besonderer Vielgestaltigkeit ist die situation (wie auch im Russischen) bei Postposition des präteritalen $\mathrm{P}_{k}$.

$\mathrm{Vgl}$. "zwei voneinander verschiedene Handlungen", mit Vorzeitigkeitsbedeutung des prät. $P_{k}$ und nur stilistischer Relevanz der Postposition:

se rekb pokaza imb roces i noze i rebra suoé. Vnzdradovase ze se urenici vidervose $\overline{\mathbf{g a}}$

J 20,20 (SS, S.175)

Beim folgenden Beleg, der grundsätzlich analog klassifiziert wird, ist die Postposition insofern relevant, als es sich, bei gesicherter Vorzeitigkeit, nicht um das zweite Wunder uberhaupt handelt, sondern um das zweite wunder nach der Ankunft in Galileja:

se paky vitoroe čjudo sztvori is prišdz ot: ijudeje vz galilejo

Die Ambiguität ${ }^{104}$ der Postposition wird anschaulich in den folgenden Beispielen; das Prädikat alleine ist negiert in: aśte zrno pošentno ne umbretr padovn...J 12,24 (SS,S.178) Anders in:

ašte ne dastz emu vastava...

L 11,8 (SS,S.178)

Die Handlung des Partizips ist mitnegiert.

Vgl. die deutsche Ubersetzung (nach Kürzinger):

"wenn er auch nicht aufstehen und ihm geben wird, weil er sein Freund ist ..."

Grundsätzlich tendiert das prät. Partizip, meist nicht ipf.. zur Vorzeitigkeit. Postposition kann insbesondere relevant werden zum Ausschluß der Vorzeitigkeitsbedeutung mit dem Ergebnis, daß nicht vorliegt Gleichzeitigkeit zweier verschiedener paralleler Handlungen wie bei präs. Partizip, sondern Darstellung

103 Weitere Beispiele in M.. S. 22

$104 \mathrm{Vgl}$. Rúziłka, SS, S. 178: "Bei Postposition wird von Fall zu Fall durch den Kontext entschieden, ob das Partizip in die Negation eingeschlossen ist." 
einer Handlung mit ihrem Merkmal. Identität zweier Handlungen, bei resultativer Bedeutung des nachgestellten (pf.) Gerund. 105 Vgl. noch als Beispiel für "eine Handlung", modifiziert durch präteritales Partizip:

i pridq podvigršese

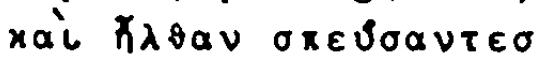

L 2,16 (SS,S.182)

szgresichz predavz krtvi nepovining Mt 27,4 (SS,S.180)

Umgekehrt schließt im folgenden Beispiel die aksl. Konstruktion mit zwei koordinierten Imperativen aus, daß etwa ein dem Imperativ nachgest. $P_{k}$ mißverstanden wirde als "zwei voneinander verschiedene Handlungen" mit Vorzeitigkeit des Partizip und Nichtguiltigkeit des Imperativ des Prädikats auch für das Partizip: intendiert ist Identität der Handlungen:

s?psi sesam. i sanidi sq krsta Mr 15,30 (SS,S.181)

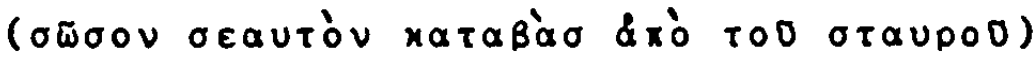

Bei Postposition ist außerdem nicht auszuschließen, daß zwischen Partizip und Prädikat überhaupt keine Beziehung, sondern nur 10se Anreihung intendiert ist. 106

Vgl. mit Koordinierung des postpositiven $\mathbf{P}_{\mathbf{K}}$ : i otrpuśt narodz vblęze va korabb. i prił̌ed vz prědĕly magdalanbsky Mt 15,39 (SS, S. 188)

Bei Postposition des präsentischen $\mathbf{P}_{\mathbf{k}}$ in Gleichzeitigkeitsbedeutung ist $2 u$ rechnen mit den semantischen Typen "eine Handlung mit ihrem Merkmal" und "zwei voneinander verschiedene Handlungen". Bei prät. Partizip in Postposition ist zu rechnen mit "zwei vonéinander verschiedenen Handlungen" bei Vorzeltigkeit und bei resultativer Bedeutung mit "eine Handlung mit ihrem Merkmal" (sowie außerdem mit loser Aneinanderreihung). Die postpositiven präteritalen meist nichtipf. $P_{k}$ des Aksl. finden ihre funktionale Entsprechung in den russischen postpositiven präteritalen pf. Gerundien, meist nach pf. Prädikat. Für postpositive prät. Partizipien aus MS 107 zur Angabe "eines

$105 \mathrm{Vgl}$. a.a.0.. S. 180ff: S. 181: "Postposition ist die bevorzugte und angemessene Stellung bei Einheit der Handlungen."

$106 \mathrm{Vgl}$. a.a.0.. S. 162, 177

$107 \mathrm{Vgl}$. ders. 62, Korr., S. 684 ; ders. 63, Typ., S. 849 
Merkmals der Prädikatshandlung" nehmen wir analog zum Aksl. resultative Bedeutung an 108

z.B für

povelomb zapisacht i potvrbdicht pečatiju moömb pečativb (MS, S. 220)

i oni ... mužïe prïoše ne zakbsněvše

(MS, S. 130)

carstvïe věčnoe óbèšta ... rekb...

(MS, S. 196) 109

i na to više upisano, kako da e věki krěpko, prisegocht i klecht se azb ... i z ... gospoinom... postavivıše ruci na suetomı evanbćeli ...

(MS, S. 236)

"Zwei Handlungen" bei Vorzeitigkeit des postpositiven prät. Partizips: 110

i koi ljubo trbgovtc. dovede kone kupivi istuge zemle

(MS, S.162)

Als Ubersetzung bietet sich adverbialer Nebensatz mit $z . B$. nachdem an, Koordinierung kommt nicht in Frage.

Hiervon zu unterscheiden sind Fälle, in denen das Partizip nur eine sehr lose Beziehung zum Prädikat hat und am ehesten im Sinne einer der Wortstellung entsprechenden zeitlichen Aufeinanderfolge $z u$ verstehen ist. Das Partizip kommt hier in seiner prädikativen Selbständigkeit einem finiten Verbum sehr nahe. Für derartige Fälle ist Koordinierung des Prädikats mit dem nachgestellten präteritalen Partizip belegt. 111

... l jubeštimb Ze jego teplě i milostivně suštostra svojegō otbčbstro obę̌ta i Žlatelnö ił̌tustimt synovostvo togó nevtzbrannö darovavo...

(MS,S.104)

$108 \mathrm{Vgl}$. M., S. 24ff; Rủǐička, SS, S. 174ff, 180ff "Die Variante der Gleichzeitigkeit des präteritalen Partizips ..."

$109 \mathrm{Zu}$ formelhaften Belegen mit rekz vgl. RüŽiðka, SS, S. $184 \mathrm{ff}$

$110 \mathrm{Vgl}$. M., S. 26ff; Rủžicka, SS, S. $187 \mathrm{ff}$

111 Vgl. a.a.O.. S. 188: "Die Abweichung spricht für die Selbständigkeit des Partizips ... Gemeinsames Merkmal aller durch Konjunktion i mit dem Hauptverb verbundenen präteritalen postpositiven Partizipien ist schließlich noch. daß sie in keinem Falle Vorzeitigkeitsbedeutung haben. Die ausdrückliche Anreihung schließt dieses temporale Verhältnis aus." 
8.4. Endstellung ohne Isolierung im Russischen

8.4.1. Aksl. gebundene Konstruktionen

Für die bisher in Postposition angeführten $P_{k}$ des Aksl. hatten wir unterschieden zwischen zwei Strukturtypen, die umgangssprachlich umschrieben worden waren als "zwei voneinander verschiedene Handlungen" und "eine Handlung mit ihrem Merkmal". In beiden Fällen liegt nicht nur syntaktische Fakultativität vor, insofern die $P_{k}$ keine durch die Valenzeigenschaften des übergeordneten Verbums geforderte Ergänzung darstellen, sondern auch kommunikativ-semantische Fakultativität, insofern die Prädikatshandlung auch bei Fehlen des $P_{k}$ als die vom Sprecher gemeinte zu identifizieren ist, wenn auch möglicherweise unvollständig dargestellt.

Hiervon $z u$ unterscheiden sind Konstruktionen, wo der "synthetische Charakter"112 der Postposition so ausgeprägt ist, daß die Prädikatshandlung ohne die Partizipialhandlung nicht mehr a]s die vom Sprecher intendierte $2 u$ identifizieren wäre; auch wenn $P_{k}$ syntaktisch fakultativ sein mag, auf der kommunikativ-semantischen Ebene liegt Obligatheit vor analog zu Restriktivität innerhalb des Nominalkomplexes.

Die extreme Ausformung dieses Funktionstyps stellt die periphrastische Form des Typs be uxe ${ }^{113}$ dar. Als umgangssprachliche Benennung für gebundene Konstruktionen in graduell unterschiedlicher Aus prägung könnte in Fortsetzung $z u$ "zwei voneinander verschiedene Handlungen" / "eine Handlung mit ihrem (fakultativen) Merkmal" dienen: "Einheit einer Prädikation".

Als Ausgangspunkt für die Veranschaulichung des graduellen Ubergangs (in den Varianten der Realisierung, nicht in der Definition des Funktionstyps) vom Funktionstyp "eine Handlung mit ihrem (fakultativen) Merkmal" $z u$ "Einheit einer Prädikation" dienen Beispiele, die wir schon bei "eine Handlung mit ihrem Merk-

$112 \mathrm{Vgl}$. RủZ̈i ka, ss, S. 141

$113 \mathrm{Vgl}$. a.a.o.. S. $202 \mathrm{ff}$ 
mal" in obligater Postposition 114 angefürt hatten. Vgl. z.B.:

smokovbniç iméałe eterz vo vinogradé svoem. vasaźdeng. i pride ište ploda na nei. i neobręte L 13,6/7 (SS,S.139)

i pride kz nimz po morju chode Mr 6.48 (SS,S.146)

Schon für derartige Beispiele gilt ansatzweise die Charakterisierung als "Konstruktionen mit potentiellem halbkopulativem Charakter des finiten verbs", 115 d.h.. das verbum ist durchaus zu selbständiger Prädikation fähig, tendiert jedoch bei Kombination mit dem postpositiven Partizip deutlich zur Einheit einer Prädikation in der Weise, daß der semantische Eigenwert des finitverbalen Prädikats reduziert wird und die Partizipialhandlung den semantischen Schwerpunkt der Aussage bildet.

$\mathrm{Vgl}$. zu Konstruktionen mit potentiellem halbkopulativem Charakter des finiten Verbs, die als affin $z$ u gebundenen Konstruktionen und als Bindeglied zwischen den oben angefürten postpositiven strukturen und der analytischen Verbalform des Typs bě ure einzuordnen sind, z.B.:

slěpr sèděase pri pqti chlopaje Mr 10,46 (SS,S.168) po v'se d'ni sédĕcho sz vami. vo crkove ǔ̌e

Mt 26,55 (SS, S.168)

Marie Ze stoěse u groba vone plaz̧̧ti se

J 20,11 (SS, S. 168)

Vgl. mit Erhaltung des semantischen Eigenwertes des Hauptverbs: vz tomb̌̌e domu prěbyvaite. ědošte i pojoł̌te. ěže sqto u nichz L 10,7 (SS,S.169)

Es besteht also grundsätzlich die nur von Fall zu Fall zu bestimmende Möglichkeit, daß sogenannte "autosemantische" Verben mit nachgestelltem Partizip in Analogie zu "gebundenen postpositiven Konstruktionen" 116 im strengen sinne fungieren, die sich auszeichnen durch die Unmöglichkeit der Paraphrase durch zwei koordinierte finite Verben, die Tatsache, daß das Partizip eine Vakanz des Hauptverbs besetzt und daß Postposition ob-

114 a.a.0.. S. $139 \mathrm{ff}$
115 a.a.0.. s. $168 \mathrm{ff}$
116 a.a.O.. S. $145 \mathrm{ff}, 188 \mathrm{ff}$ 
ligat ist.

Vgl. mit Phasenverb als Prädikat, synonym mit Partizip kann Infinitv auftreten: 117

Ěko Že prěsta gle. rě̌e simonu L 5,4 (SS,S.189)

Partizip, das aus Gründen der semantischen Geschlossenheit an das Hauptverb gebunden ist: 118

... da bišs sq avili Ekom postę̌ste Mt 6,16 (SS,S.192)

i ě̌e aste mnito sq imy. vizeto bqdetz otz nego

Mt 25,29 (SS, S.192)

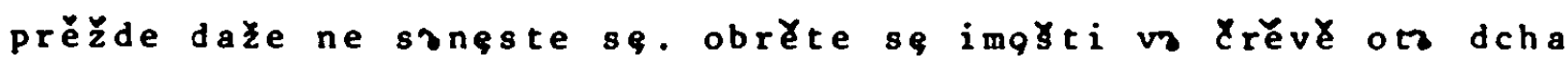
st a

Mt 1.18 (SS,S.193)

Vgl. demgegenüber mit "Eigenwert" des fin.Prädikats obrëte se: 119 éko $\overline{s m z}$ moi st. mrotrs bě i ožive. izgybr bě i obrětese

L 15,24 (SS, S. 193)

Vgl. zur Relativität solcher Kennzeichnungen wie autosemantisch, potentiell halbkopulativ u.ä. auch die Belege aus MS $z u$

obrěsti se und verwandten Konstruktionen:

ašte li kotori óbrěštetu se siju zapovědı prěstupaje, da na-

kazoujett se (MS.S.14)

Infolge der minimalen untergeordnet prädikativen selbständigkeit des Partizips kann in derartigen Konstruktionen $u . U$. auch Attributivität vermutet werden:

öbrěte li se kto clověkb zolb ili ödo moicht ili ödb vašchb potvarajesije, ou tomo da ně zledi nikoere...

(MS, S. 20)

Vgl. analog zur obigen gebundenen Konstruktion:

kto 1 jubo boudet prêtvaraje sija, ... da ...

(MS, S.15)

Vgl. mit präteritalem Partizip:

117 In der Folge verdrängte der Infinitiv die Konstruktion mit Partizip, vgl. Rủzička, SS, S. 191; zu Zukunftsumschreibungen mit dem Inf. im Aksl. vgl.ausführlich Birnbaum 58

$118 \mathrm{Vgl.} \mathrm{Rư̌iČka,} \mathrm{SS,} \mathrm{S.} 191 \mathrm{ff}$

$119 \mathrm{Vgl}$. a.a.O.. S. 194: "Für eine im Slavischen vor Einsetzen der altslavischen Tradition $z u$ vermutende Konstruktion des Typs obresti sq-Partizip läbt sich allerdings schwer etwas über den Grad der Trennung oder Zusammengehörigkeit von Hauptverb und Partizip sagen.": vgl. M.. S. $27 f f$ 
ako 1 i se kto naide prodavb (MS, S.17)

Statt des Partizips mit Infinitiv:

kto li droznetb razoriti

(MS, S. 111)

ałte li kto i öbreß̌tet se takovyi ouchištreniem diavolskimb razoriti

(MS, S. 132)

Statt der gebundenen Konstruktion kann einfaches finites Verbum auftreten:

kto ... potvoritb

(MS , S.135)

Als Beleg für den Ubergang von sogenanntem autosemantischem obrésti se zu halbkopulativem können möglicherweise die folgenden Beispiele dienen:

obrěte li se kto i drtzneto potvoriti se (MS,S.27)

kto li se obrete i droznetb potvoriti (MS,S.213)

Den evidenten Endpunkt des durch die oben angeführten Belege veranschaulichten graduellen Ubergangs vom Typ "eine Handlung mit ihrem Merkmal" zu "Einheit einer Prädikation" bei postpositivem $P_{k}$ markiert die periphrastische Form ${ }^{120}$ des Typs bě uče. bestehend aus Hilfsverb, das nicht in der Nullform auftreten kann, und Partizip, das nicht als Prädikatsnomen, sondern als Teil einer analytischen Verbalform fungiert, d.h.. hier liegt nicht mehr zweibasige struktur zugrunde wie bei Postposition, soweit sie von uns umschrieben wurde als "eine Handlung mit ihrem (fakultativen) Merkmal".

Vgl.:

Bé že urę na edinomb ot sznzmištb v sqboty

L 13,10 (SS, S. 204)

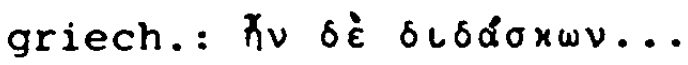

Partizip und Hilfsverb sind nicht voneinander zu trennen, es besteht funktionelle Änlichkeit mit dem Imperfekt učaaše und die Möglichkeit der Ersetzung durch diese finite Form ohne Veränderung der syntaktischen struktur.

Vgl. noch als Beispiele für die periphrastische Form:

irodz Že videvz isa. radz bystz zĕlo. bě bo Žlèe otz mnogavre-

120 Vgl. Rủžið̌ka, ss, S. 202ff: vgl. auch Björk 40; M., S. $29 \mathrm{ff}$ 
mene vidêti i

L 23,8 (SS,S.209)

Vgl. mit Hilfsverb im Präsens:

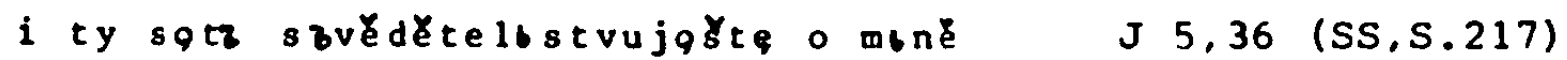

Hilfsverb im Imperativ:

bqdi uvȩtaje sq st sqpurbmb svoimb skoro Mt 5,25 (SS,S.218)

Demgegenüber mit peripherer Prädikativität des postpositiven Partizips:

i bèachq viinq. vz crkzve. pojofte i blagoslovęste ba

Vgl. Čech.:

L 24.53 (SS, S.204)

A byli vỉdycky v chrámé, chválíce a dobrořeč́ce Boha

(mit Isolierung!)

Die angeführten aksl. Belege veranschaulichen in Postposition grundsätzlich drei mögliche Funktionstypen der Kurzform des Partizips (von der attributiven abgesehen). "zwei voneinander verschiedene Handlungen"/"eine Handlung mit ihrem Merkmal"/ "Einheit einer Prädikation".

Der letzte Typ findet seine eindeutige Realisierung in der periphrastischen Form des Typs bé u\&̧, für die keine zweibasige Ausgangsstruktur zugrundegelegt werden kann, eben weil hier nicht mehr periphere Prädikativität des Partizips vorliegt. Unser Vorschlag lautet, auch die mehr oder weniger gebundenen Konstruktionen analog $2 u$ interpretieren. Die Begründung hierfür ist nicht syntaktischer, sondern, in der Konsequenz der vorgeschlagenen Umschreibung der untergeordneten Prädikativität, kommunikativ-semantischer Art: Auch bei potentiell selbstwertigen Verben ("autosemantische" Verben) ist mit der durch Beispiele $z u$ veranschaulichenden Möglichkeit $z$ u rechnen, daß sie gemäß der kommunikativen Intention des Sprechers zusammen mit der postpositiven Kurzform des Partizips als "Einheit einer Prädikation" fungieren, d.h.. nur Prädikat und Partizip zusammen bezeichnen die vom Sprecher gemeinte Handlung, ohne das Partizip ist diese nicht zu identifizieren, obwohl syntaktisch auch Prädikat ohne Partizip möglich wäre und damit auch syntaktische Ableitung von zweibasiger Ausgangsstruktur, womit der funktionale Unterschied hinsichtlich der untergeordneten Prädikativität des 
postpositiven Partizips jedoch verwischt wïrde.

Dessen strukturelle Relevanz. wird sichthar durch die Unmöglichkeit der Paraphrase anhand Koordinierung sowie im Russischen und Skr. durch obligate Nichtisolierung. Im Aksl. steht demgegenüber das Kriterium der Isolierung nicht zur Verfügung.

Von cen oben angeführten postpositiven $P_{k}$ als Teil der periphrastischen struktur be uce u.ä. zu unterscheiden ist die Langform des Partizips als Prädikatsnomen: 121

iméjei zapovědi moje. i szbljudaje je. tz estz ljubei me.a ljubei me. vizljublenz bodetz ocemb moimb J 14,21 (SS,S.276)

St estb krlstei dchom: strim! J 1.33 (SS,S.311)

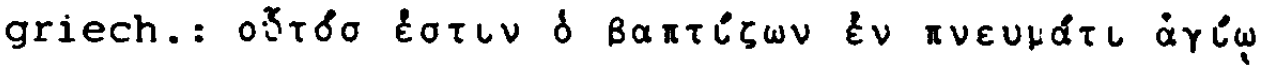

Das Partizip als Prädikatsnomen hat identifizierende Funktion. Vgl . demgegenüber:

t. estr l jube bzw. ti 1 jubitz

sowie griech.; hv $\delta \iota \delta \& \sigma x \omega \nu$

8.4.2. Skr. fokussierte Gerundien

Auch für das Skr. hatten wir unterschieden zwischen postpositiven Gerundien, bei denen Negation des Prädikats alleine möglich ist, nicht aber Paraphrase durch Koordinierung, und anderen, für die umgekehrt Paraphrase durch Koordinierung plausibel und die Negation des Prädikats alleine nicht möglich ist. In beiden Fällen, bei "zwei voneinander verschiedene Handlungen" und bei "eine Handlung mit ihrem Merkmal", treten isolierte und nichtisolierte Belege auf. Nun findet sich außerdem eine Gruppe von Beispielen, die üblicherweise nicht exakt getrennt wird vom Typ "eine Handlung mit ihrem Merkmal", sondern

$121 \mathrm{Vgl.M.,} \mathrm{S.} \mathrm{73;} \mathrm{Rưžiłka,} \mathrm{ss,} \mathrm{S.} \mathrm{279ff,} \mathrm{311ff,} \mathrm{S.} \mathrm{280:}$ "Nichtprädikativität des Vollpartizips bedeutet, daß die Handlung des Partizips nicht prädiziert werden kann. Die Handlung des Partizips kann nicht Inhalt der Prädikation. d.h. Teil des in ihr berichteten Ereignisses sein. Das schließt aber nicht aus, daß ein Vollpartizip Teil des prädikats, nämlich Prädikatsnomen sein kann." 
mit einer an "Varianten" der Kategorie "adverbiale Funktion" orientierten Terminologie umschrieben wird als "modal" u.ä.. Isolierung tritt hier nicht auf. Erweiterung des Gerund fehlt weitestgehend, in meinem Material finden sich nur präs. Gerundien, während in den oben angefürten Belegen fur Postposition (selten) auch prat. Formen auftraten.

Für diese Gruppe gilt wie beim oben angegebenen Typ "eine Handlung mit ihrem Merkmal " Unmöglichkeit der Negation des Prädikats alleine, außerdem jedoch Unmöglichkeit der Paraphrase durch Koordinierung. Im Einzelfall ist Ambiguität nicht auszuschließen, doch beeinträchtigt dies nicht die grundsätzliche Unterscheidbarkeit des Funktionstyps, dessen Spezifik darin liegt, daß hier das Gerund rhematischer Höhepunkt innerhalb des Prädikatskomplexes ist ("Fokussierung").

Vgl.:

on govori šaleći = On govori śaljivo (Pełikan 59,5.91)

Danas sam rutao stojeci (Belić 69,s. 193)

Rucao sam stojeći = Ručao sam stojecke (Musić 35.S.135)

On zaraduje trgujuci = On zaraduje trgovanjem

(Pesikan 59,5.92)

On nas zabavlja šaleći $=\ldots$ šalenjem, $\begin{array}{r}\text { šalom } \\ \text { (Pešikan 59,5.91) }\end{array}$

Die Fokussierung signalisiert die Tatsache der kommunikativsemantischen obligatheit, $d . h$. nur zusammen mit dem Gerund ist die Prädikatshandlung die vom Sprecher intendierte, während das Gerund bei den Typen "eine Handlung mit ihrem Merkmal" und "zwei voneinander verschiedene Handlungen" kommunikativ fakultativ ist. Syntaktische Fakultativität ist nur eine notwendige. nicht aber hinreichende Bedingung für kommunikative Fakultativität. In der Sekundärliteratur wird in Zusammenhang mit kommunikativ obligaten Gerundien gerne verwiesen auf die "schwache" und ergänzungsbedürftige Semantik des übergeordneten Prädikats, grundsätzlich ist aber bei den hier angeführten Belegen sehr wohl auch Gebrauch des Prädikats ohne Erweiterung z.B. durch Gerund denkbar.

Fügungseigenschaften des übergeordneten Verbums und seine im 
allgemeinen schwer zu objektivierende "reduzierte" Semantik können Anhaltspunkte liefern für die Spezifizierung; jedoch ist auch bei"autosemantischen" Verben mit allen drei Funktionstypen $z u$ rechnen, so daß sich die (fließende) Unterscheidung "synsemantisch" : "autosemantisch" u.ä. selbst nicht als Definitionsgrundlage eignet.

Die Umschreibung des fokussierten kommunikativ obligaten Funktionstyps als "Einheit einer Prädikation" 122 in Analogie $z$ u den oben angefuihrten aksl. "gebundenen" Konstruktionen im weiteren Sinn ist zusätzlich motiviert durch Formen der wechselseitigen Verschränkung von Gerundial- und Prädikatsstruktur wie z.B. in: Siromah otide plaćući u pečinu i kaže sve šćeri

(Musić 35.S.143)

Vojnici veselo idahu pjevajući

(ReSetar 22.S.181)

Vgl. noch:

Priznao mi je plačući i moleći za oproštaj (Schmaus,S.167)

I tako on ode a mati ostane plačući (Musić 35,S.147)

Auf die funktionale Affinität kommunikativ obligater Gerundien zu bestimmten A.dverbien oder Prädikatsnomen, für die ja z.B. Rúžička $66^{123}$ auch nicht den "synsemantischen" Charakter des Prädikats als Bedingung anführt, verweist die folgende Paraphrase bei Peక̌ikan:

On čeka tǔ̌no i ćutljivo = On čeka tužan i ćutljiv (Pešikan 59.5.92)

8.4.3. Russ. nichtisolierte Strukturen

Während im Skr. bei Postposition und Nichtisolierung mit den drei Typen "zwei voneinander verschiedene Handlungen"/"eine Handlung mit ihrem Merkmal"/ "Einheit der Prädikation" zu rech-

122 Musić 35 faßt postpositive Gerundien unseres Typs "eine Handlung mit ihrem Merkmal", Gerundien nach "synsemantischen" Verben und periphrastische, nicht mehr "zweibasige" Strukturen des Typs kad su bili putem putujuci ... (Musić 35,S. 147) als "prädikativ" zusammen

$123 \mathrm{Vgl}$. Rư̌ička $66, \mathrm{~S}, 57 \mathrm{ff}$ 
nen ist, hatten wir für das Russische für "zwei voneinander verschiedene Handlungen" (Satzbezug) Isolierung auch bei Postposition als obligat festgestellt. Außerdem wurde bei postponierten isolierten strukturen der Typ "eine Handlung mit ihrem Merkmal" (Bezug auf das Prädikat) nachgewiesen.

Während für das Russische die Signifikanz der Isolierung allgemein betont wird, ohne daß sie präzisiert wirde, sieht auch Weiss für das Polnische die Isolierung nicht als gleichermaßen relevant an. Weiss nennt in Kulissenfunktion uberwiegend isolierte Formen (in Anfangs- und Endstellung): dies gilt jedoch nicht ausschließlich.

Auch für

Stuchat zasypiajac

(Weiss $77,5.313$ )

wird von Weiss Kulissenfunktion angenommen. 124 Evident ist Nichtisolierung bei Fokussierung. Bei dem von Weiss nicht ausführlich erörterten Typ "eine Handlung mit ihrem Merkmal" scheint Isolierung und Nichtisolierung möglich zu sein.

Soweit für das Russische das Satzglied "prädikativer Determinant" in einer isolierten und einer nichtisolierten variante angeführt wird, tritt das Gerund als "Halbsatzkonstruktion" üblicherweise nur isoliert auf. Der begrifflichen Unsicherheit hinsichtlich der Signifikanz der Isolierung entspricht die Vielfalt der Gesichtpunkte, die als Bedingung für Nichtisolierung russischer Gerundien genannt werden, so Nichterweiterung, Verlust der "Handlungsbedeutung", Verlust der untergeordneten Prädikativität, Ubergang in eine andere Wortart, insbesondere in die Klasse der Adverbien, aber auch in die der Präpositionen, Stellung in der aktualen Gliederung, syntaktische Funktion als adverbiale Bestimmung u.a.m.

8.4.3.1. Koordinierung von Gerund und Adverb

Als eine mögliche Ursache für die Nichtisolierung von Gerundien

124 U.E. liegt hier allerdings nach den von uns erörterten Kriterien eher vor "eine Handlung mit ihrem Merkmal" (Bezug auf das Prädikat) 
insbesondere, aber nicht ausschließlich, in der Position nach Prädikat ist Koordinierung mit nichtisoliertem Adverb zu beobachten, ohne daß deswegen Ubergang des Gerund in die Wortart Adverb anzunehmen wäre.

Vgl. z.B.:

Klim Samgin šagal po ulice bodro i ne ustupaja dorogu vatrečnym 1 judjam

(Rudnev 63,S.177)

Devuška govorila bystro i ne umolkaja (Babby 75,5.5)

Ona otveciala botom i potupiv golovu (Babby 75,5.5)

Oni vytaskivali bol'nych na matracach ili prosto vzjav pod myski (Rudnev 63,5.177)

"Kak Speranskij" - pribavil Bazarov posle nebol'šogo molčanija i skriviv guby

(Rudnev 63, S.177)

Wir unterstellen, daß hier die Konjunktion i Elemente gleicher Rangordnung in der Konstituentenstruktur verknüpt. Bei Paraphrase der Gerundialstruktur durch koordiniertes finitverbales Prädikat wird allerdings diese Gleichordnung mit der Adverbialstruktur aufgegeben, diese erscheint dann alleine als Bestimmung des finitverbalen Prädikats der Ausgangsstruktur, dieser ganze Komplex wird mit dem zweiten, durch Paraphrase erzeugten finiten Prädikat koordiniert.

Es handelt sich bei den Adverbien und den koordinierten Gerundialstrukturen um weder syntaktisch noch kommunikativ obligate Strukturen, die wohl als "eine Handlung mit ihrem Merkmal" zu klassifizieren sind und grundsätzlich als Indiz dafür dienen können, daß im Russischen auch dann Nichtisolierung des postponierten Gerund nicht ganz auszuschließen ist, wenn nicht Fokussierung vorliegt. Zugleich veranschaulichen diese Beispiele, daß der Hinweis auf einen zu rekonstruierenden Konstituentensatz mit identischem Subjekt noch nicht ausreicht zur spezifizierung des jeweiligen Funktionstyps im Satz.

\subsubsection{Adverbialisierung?}

"Adverbialisierung" des Gerund wird häufig als Bedingung der 
Nichtisolierung angeführt, ohne daß immer deutlich wiirde, ob damit eine syntaktische Funktion oder der Ubergang in eine wortart gemeint ist.

Was den Ubergang in die Wortart Adverb betrifft, so ist hier wohl eine exakte Definition ähnlich schwierig wie im Falle der Substantivierung und Adjektivierung, und es ist mit Ubergangserscheinungen $z u$ rechnen. Ein wesentliches Kriterium für den Nachweis des fortbestehenden Kontakts zum Verbalparadigma, nicht jedoch für die Spezifik des Funktionstyps, ist die Rekonstruktion eines (Konstituenten-)Satzes mit Umwandlung des Gerund zum finitverbalen Prädikat. 125

In den Handbüchern erfolgt weniger eine Definition der Adverbialisierung als (im einzelnen unterschiedliche) Aufzählung von angenommenen "typischen" Beispielen der Adverbialisierung. für die andererseits z.T. auch die zusätzliche Möglichkeit des "gerundialen Gebrauchs" betont wird, so z.B. bei Pičugov 73, S. 37 für stoja,sidja, leža, molča, šutja, nechotja, ne spěsa, ne gljadja. 126

Das distributive Kriterium der (Nicht-)Erweiterung hilft dabei nicht weiter, nachdem auch unstrittige Gerundien ohne Erweiterung und umgekehrt "adverbialisierte" Gerundien z.B. in phraseologischen Wendungen wie slomja golovu,spustja rukava usw. mit Erweiterung auftreten.

Semantische Umschreibungen wie "Verlust der Handlungsbedeutung, Angabe eines Merkmals der Prädikatshandlung, enge Verbindung mit dem Prädikat" u.à. bleiben notwendig subjektiv und unter liegen grundsätzlich der Kritik an einer semantischen Definition der Wortarten (s.o.).

Entgegen Gvozdev ist u.E. z.B. nicht recht einzusehen, daß nur im ersten der beiden folgenden Beispiele "Handlungsbedeutung" vorliegen soll:

Chudoźnik risuet, stoja na podmostkach

Chudožnik risuet stoja

$125 \mathrm{Vgl}$. Weiss 77, S. 376, Rudnev 68, S. 157, M., S. $12 \mathrm{ff}$

$126 \mathrm{Vgl}$. z.B. auch AnaniC 72, S. 53ff, 57ff: Morison 59, S.36ff Gnevko 75, S. 83ff; Rudnev 63, S. 175ff; Gvozdev I 73, S. 387ff: Paffen II, S. 360ff 
Wirklich zuverlässige Kriterien für den Ubergang in die Wortart Adverb könnten allenfalls abweichende Betonung und die morphologische Form sein. So sind die (genuin russischen) Formen auf $-u X_{i} /-j u X_{i}$ wohl in den allermeisten Fällen als Adverbien anzusprechen. Vgl. z.B. die feststehende Wendung: dolgo li umejuči? 127

V polnoč' Grigorij kradučis' podošel k stanu, stal šagach v des jati ...

(Gnevko $75, \mathrm{~S} .87$ )

Das wohl am häufigsten für "abweichende Betonung" genannte Beispiel ist mólદ̌a im Unterschied zum Gerund molčá. Isačenko allerdings beurteilt auch derartige Fälle mit zurückhaltung. 128 Vgl. 2.B.:

Inogda on (ded) dolgo i mólča razgljadyval menja, okrugliv gla$z a, k a k$ budto vpervye zametiv

(Ananič 72,S.58)

Eindeutig bezeichnet ist die Adverbialisierung und damit die Unmöglichkeit der Umwandlung zu finitverbaler Prädikation mit einem Agens, das referenzidentisch ist mit dem subjekt des Hauptsatzprädikats für departizipiale Adverbien 129 in unterschiedlicher Funktion.

Vgl. z.B.:

Ego rasskaz dejstruet vozbuždajušče na menja

(Paffen II,S.352)

Ona voprošajušče gljanula na nego (Paffen II,S.352)

otnošenija mne išcerpyvajušče jasny (Paffen II,S.352)

Rabota idet, konečno, ne blestjašče (Morison 59,S.51)

Als Indiz für Adverbialisierung wird der Gebrauch in "festen

Wendungen" angeführt; vgl. z.B.:

Stepan ... brosilsja slomja golovu v palisadnik

(Rudnev 63,5.177)

127 Isačenko 75, S. 29: Paffen III, S. 209: Gnevko 75, S. 87: Rudnev 63, S. 175: nach Morison 59, S. $34 \mathrm{ff}$ ist für derarttge Formen umgangssprachlich auch nichtadverbialer Gebrauch möglich

$128 \mathrm{Vgl}$. Isačenko 75, S. 333, Anm.1; $\mathrm{zu}$ weiteren Beispielen mit abweichender Betonung s. S. $328 \mathrm{ff}$

129 Vgli hierzu auch Weiss 77, S. 374ff zum Poln.: zum Russ. vgl. Isacenko 75, S. 325, Jacobsson, Gö. 69, S. 82 
On stojal ne dvigajas'

(Paffen II,S.369)

Lošadi stojali ponurja golovu i izredka vzdragivali

(Gnevko,S.85)

On rabotal ne pokladaja ruk

(Gnevko, S.84)

On sidit sloza ruki

(Mulisch 75, S. 206)

On rabotaet spustja rukava

(Mulisch 75,5.209)

Vgl. in unpersönlichen Konstruktionen:

Prichoditsja stojat' povesja golovu (Rudnev 63,s.179)

Nado bylo skrepja serdce primenjat'sjak novym obstojatel'stram (Paffen III,S.209)

Für die Funktion solcher metaphorischer und als solcher nicht teilbarer Wendungen ist im Grunde nicht entscheidend, ob man sie auf einen Konstituentensatz mit identischem Subjekt zurückführt, oder ob man sie im Lexikon aufführt. In jedem Fall nennen sie ein wesentliches Merkmal der Prädikatshandlung, die ohne dieses Merkmal nicht als die vom Sprecher gemeinte zu identifizieren wäre.

Von derartigen Ausdrücken müssen, auch wenn sie gelegentlich ohne Unterschied als "feste Wendungen" zusammengefaßt werden, Gerundien in absoluter stellung unterschieden werden. Ist für die oben aufgefuhrten Wendungen fraglich, ob ein (gegenüber dem satzschließenden Prädikat identisches) subjekt zu rekonstruieren ist, wobei in jedem Fall Funktion innerhalb des Satzes vorliegt, Bezug auf die Prädikatshandlung, so stehen die folgenden Gerundien außerhalb des Satzes: 130

Sobstuenno govorja, vy pravy

(Mulisch 75.5.210)

Sudja po naruznosti, vy molože menja

(Mulisch 75.5.210)

Pravdu govorja, nam nelegko véti dni (Svetlik 70,S.270)

Logičeski rassuždaja, on skazal sejčas glupost'

(Svetlik 70,5.270)

Voobščěce telo ego, sudja po matovomu, čeresčur belomu cretu šei, malen'kich puchlych ruk, mjagkich pleč, kazalos'sliškom iznežennym dlja mužtin

(Ruaner 63 , S.178)

Vyražajas' figural'no, u menja byvalo takoe oščuščenie, slovno' - greche razglagol'struet angel (F,S.180)

$130 \mathrm{Vgl}$. Weiss $77, \mathrm{~S} .293 \mathrm{ff}$ 
Derartige Konstruktionen sollen hier nicht ausführlich erörtert werden. Jedenfalls fält es schwer, sie als nichtprädikativ zu bezeichnen, obwohl ein mit dem satzschließenden Prädikat der Oberflächenrealisierung des Satzes identisches Subjekt nicht $z$ u rekonstruieren ist. Ein vom Subjekt der satzschließenden Prädikation verschiedenes unpersönliches Agens, identisch letztlich mit dem Sprecher, kommentiert weniger den Inhalt der Äußerung als die Tatsache, die Art und Weise der Außerung selbst 131 (vgl. deutsch so-zusagen).

Explikation könnte erfolgen durch einen zu tilgenden Hypersatz, 132 der sich umschreiben ließe etwa in der Form Indem ich in einer bestimmen Weise urteile, eine Aussage mache ..., sage ich, daß... (Hauptsatz)

\subsubsection{Fokussierung}

Als Beispiele für "Grenzfälle" der Adverbialisierung könnten die folgenden Belege dienen:

On otvetil ne dumaja, ̌sutja (Mulisch 75.5.209)

On ċašče vsego rabotaet stoja (Mulisch 75, S.209)

Vgl. demgegenüber mit Erweiterung und Isolierung:

On rabotaet, stoja na lesenke

(Mulisch 75, S.209)

On šel ne ogladyvajas'

(Žaža 64,S.185)

Mit Isolierung:

On toroplivo šel, ne ogladyvajas'

(ŽaŽa $64,5.185$ )

Kuznečiki strekotali ne umolkaja: Kuznečiki dolgo strekotali, ne umolkaja

(Žaža 64, S.185)

Davydov stojal ne śeveljas', to chmurjas', to udivilenno podnimaja vygorev̌sie brovi

(Gnevko 75, S.84)

Für die Funktionsbestimmung der hier infragestehenden nichtisolierten Gerundien in der Distribution $(\mathrm{N}+) \mathrm{V}+\mathrm{Ger}$ ist $\mathrm{U} . \mathrm{E}$. im

$131 \mathrm{Vgl}$. Weiss 77, S. 296; Bartsch 72, s. 28ff, 65ff, 67

$132 \mathrm{Vgl}$. Weiss 77 , s. $299 \mathrm{ff}$ 
Grunde nicht entscheidend, ob man noch einen Konstituentensatz zugrundelegt oder diese Formen im Lexikon auffüht, sondern daß sie fokussiert sind. 133

Die Tatsache der Fokussierung ist diesen Belegen gemeinsam mit den folgenden, für die auch in den Handbuichern nicht Adverbialisierung angenommen wird und die sich in ihrer Funktion nicht grundsätzlich von den oben angeführten Beispielen unterscheiden:

On šel s trudom peredvigaja pravuju nogu

(Gvozdev II $73,5.163$ )

Spivka zila ne zadevaja, ne poučaja ego

(Gvozdev II 73,S.163)

Poslednjuju not' on provel ochaja i stonaja i izmučil Ninu Aleksandrovnu...

(Gvozdev II 73,S.163)

S nedelju on prožil čego-to ožidaja (AnaniC,s.53)

Devolka čitala prislušivajas'

(sint.r.j..s.101)

Ona slušla menja ulybajas'

(Svetlik,S.246)

Ja priß̌el k vam poobedav

(Brave 40,S.25) 134

Nikita sidel potupivłis'

(Paffen II.S.209)

Pionery idut razgovarivaja

(Ananič, S. 54)

Für alle diese fokussierten Beispiele gilt Unmöglichkeit des Prädikats alleine und im Unterschied zu dem Typ "eine Handlung mit ihrem fakultativen Merkmal" außerdem Unmöglichkeit der Paraphrase durch Koordinierung; unabhängig davon, ob man im Einzelfall Ubergang in die Wortart Adverb unterstellt, sind diese Gerundien syntaktisch fakultativ und kommunikativ-semantisch obligat, insofern nur zusammen mit dem nichtisolierten Gerund die Prädikatshandlung als die vom Sprecher intendierte zu identifizieren ist.

Die angeführten Beispiele zeigen, daß auch im Russischen $z u$ rechnen ist mit nichtisolierten Gerundien und daß die Nichtisolierung nicht hinreichend $z u$ begründen ist durch den Ubergang

133 Zum Fokuscharakter derartiger strukturen vgl. Žaža 64, S.185: vgl. auch Gvozdev II 73,S. 163

134 Brave a.a.o. betont, daß der zitierte Satz annähernd äquivalent ist mit $J a$ priłel $k$ vam sytyj, nicht aber mit Poobedav,ja prišel $k$ vam = Ja poobedal i posle étogo prišl k vam. 
in die Wortart Adverb. Insbesondere zeigen dies, bei deutlichem Uberqewicht der ipf. präs. Gerundien, ${ }^{135}$ die angeführten prät. Formen, für die auch in den Handbüchern nicht Adverbialisierung vermutet wird. Im Hinblick auf die Gesamtmenge des Materials an Partizipial- und Gerundialstrukturen sind derartige nichtisolierte Beispiele relativ selten.

Die semantischen Hinweise $z u$ "Adverbialisierung" beschreiben nicht die Spezifik der Wortart Adverb, sondern die funktionale Spezifik fokussierter Gerundialstrukturen. ${ }^{136}$ (Im übrigen ist ja auch mit isolierten nichtfokussierten nichtgerundialen Adverbialstrukturen $137 \mathrm{zu}$ rechnen). Die Fokussierung konstituiert den Funktionstyp, den wir bereits bei den oben aufgefuhrten aksl. und skr. Belegen umschrieben hatten als "Einheit einer Prädikation".

Maßgeblich für die Konstituierung dieses Funktionstyps sind grundsätzlich nicht Fügungseigenschaften (Nichterweiterung des Gerund) und nicht semantische Relationen, die vermutet werden aufgrund der lexikalisch-semantischen Spezifik der beteiligten Komponenten, jedenfalls nicht im Sinne einer hinreichenden Bedingung. Die Fokussierung liegt der Unmöglichkeit der Paraphrasierung ${ }^{138}$ nichtisolierter Gerundien auch nach "autosemantischen" Verben zugrunde. Eine syntaktische Begründung einer Gruppe "adverbähnlicher" Gerundien dürte kaum zu geben sein; ihre Affinität $z u$ bestimmten Arten von Adverbien ("modal" u.ä.)

$135 \mathrm{Vgl}$. auch Mulisch 75, S. 209; ŽaŽa 64, S. 182; Ananič 72, S. 52ff; Vinogradov 47, S. 392; zum Skr. analog z.B. Musić $35, S .136 \mathrm{ff}$

$136 \mathrm{Vgl}$. 2.B. Rudnev 63, S. 177ff, ders. 59, S. 67: "Ne podležat obosobleniju taǩ̌e deepričastija, kotorye vmeste $s$ verbum finitum sostavljajut odno ponjatie ili vystupajut $v$ roli imennoj časti sostavnogo skazuemogo, vyražaja smysl skazuemogo vyskazanija.": vgl. ders. 68, S.156ff; Sviblova 62, S: 177; Križková 69, PO, S. 32; Gnevko 75, S. 84 ; Ananic 72 , S. $53 \mathrm{ff}, 57 \mathrm{ff}$

$137 \mathrm{Vgl}$. 2.B. Gabka 76, S. 218

$138 \mathrm{Vgl}$. z.B. Weiss 77, S. 276 und S. $339 \mathrm{zu}$ Gerundien in "akzessorischer" Bedeutung 
ist begründet durch die Fokussierung. 139

Dieser fokussierte Gebrauch von Gerundialstrukturen kann nicht als Beleg für Adverbialisierung dienen, sondern möglicherweise als ihr Ausgangspunkt. Indiz für abgeschlossenen Ubergang in die Wortart Adverb ist dann eventuell der Gebrauch der nichtisolierten Gerundialform auch in anderer als der fokussierten Position.

Vgl. 2.B.:

Slezy i ulybka, molta protjanutaja ruka (Rudnev 63,5.175)

Polčasa spustja Arkadijs Bazarovym sořli vostinuju

(Rudnev 63,S.175)

Aber auch hier ist z.T. Homonymie mit strukturen, für die Ableitung von finitverbaler Prädikation möglich ist (statt Aufführung im Lexikon) nicht auszuschließen.

Die durch Fokussierung begründete funktionale spezifik geht verloren, wenn z.B. Schalller ${ }^{140}$ sogenanntes Prädikatsnomen als

Temporalsatz paraphrasiert:

Ja priśel na bereg odnimiz poslednich /

Ja priśel na bereg, kogda ja byl odnimiz poslednich

(Schaller 71, S.165)

Nach Auskunft von Muttersprachlern ist der zweite Satz nicht als Paraphrase des ersten akzeptabel.

Der Funktionstyp "Einheit einer Prädikation" ist grundsätzlich möglich bei Verben, die ohne Erweiterung fungieren können (s.0.). Vgl. z.B.:

Davydov stojal ne seveljas' (Gnevko,S.84)

Die extreme Ausformung dieses fokussierten Typs stellt der Ge-

$139 \mathrm{Vgl}$. auch Pala in Daneš 74 (ed), S. 205ff: "We have already mentioned the so-called inherent adverbial modifiers, which are, in fact, semantic supplements of verbs, according to Kopecný (K.1958), "ad-verbial attributes", expressing the degree (intensitiy) or qualitiy of a verbal action. As for FSP, it means that they are -in the same way as attributive expressions in NP groups- communicatively more dynamic than their headwords (verbs) and together with them (or under special circumstances only by themselves) represent an $R$ (Svoboda 1968). This fact is also reflected by the word order." vgl. a.a.0.. S. 199ff; Uhlírová in Daneš 74 (ed), S.210ff

$140 \mathrm{Vgl}$. Schaller 71, S. $163 \mathrm{ff}$ 
brauch von Gerundien alleine oder mit Kopula in der Position der satzschließenden Prädikation dar, wie sie insbesondere in Dialekten und der miindlichen Rede, aber doch auch (selten) in der Schriftsprache 141 belegt sind. Hier liegt nicht nur kommunikativ-semantische, sondern auch syntaktische obligatheit vor.

Derartige Konstruktionen werden insbesondere von pf. Verben gebildet und treten auf mit den Endungen $-v,-v \grave{s} i,-\not \zeta i, ~ d i a l$.

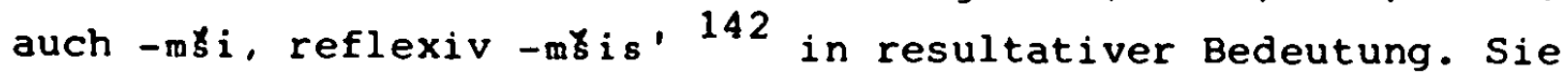
lassen sich einordnen als die maximale Ausprägung des postpositiven nichtvorzeitigen Gebrauchs von $\mathrm{pf}$. aksl. $\mathrm{P}_{\mathrm{k}}$ bzw. russ. Gerundien.

Vgl. z.B.:

Ja segodnja ves' den'ne emši (Isą̌enko 75,S.331)

Vy vot vljubivšis', a Ěem èto končitsja? (Gill'̌enok,s.27)

On vypiv̌si

(Paffen II,S.376)

On byl vypivłi

(Paffen II, S.376)

On prišel vypiv̌si

(Brave 40,5.27)

Počtennyj starec étot postojanno byl serdit i vypivši ili vypivši i serdit vmeste

(Rudnev 59, S.63)

Chodil na večcerinki, vyučilsja tancevat'kadril'i pol'ku, po prazdnikam vozvraščalsja domoji vypivši i vsegda sil'no stradal ot vodki

(Rudnev 59, S.67)

On ušedr̈i

(Brave 40,5.27)

Eine exakte Grenze zwischen Belegen wie dem letzten mit dem Gerund in der Position des satzschließenden Prädikats, für den evidenterweise nicht mehr Ableitung von einer zweibasigen Struk-

$141 \mathrm{Vgl}$. Rudnev 59, S. 62ff; Ananič 72, S. 64 nennt den Gebrauch des Gerund als Prädikat als häufiger. stilistischen Fehler, den der Lehrer $z u$ verhindern habe, 2.B. in Ja davno zabolevsi: zum Skr. vgl. Belić 69, S. $194 \mathrm{ff}$

$142 \mathrm{Vgl}$. Gilčenok 73, S. 27: Isačenko 75, S. 331; Paffen II, s. $375 \mathrm{ff}$; außer Betracht sollen hier dialektale Verwendungsweisen bleiben, in denen die aktive Form des Gerund auch reflexive oder passivische Bedeutung haben kann, vgl. z.B.: Gusi už prignavši domoj (Paffen II,S.376) = Die Gänse sind schon heimgetrieben; allgemein zu Partizipialkonstruktionen in russ. Dialekten vgl. Kuz'mina/Nemčenko 71 
tur infragekommt, ad hoc als "synsemantisch" bezeichneten Strukturen und fokussiertem Gerund bei "autosemantischen" Verben ist nicht $z \mathrm{u}$ ziehen.

Auch für fokussierte Gerundien nach sogenannten autosemantischen Verben kann auf der kommunikativ-semantischen Ebene nicht mehr gut von einer "Beziehung" zwischen uber- und untergeordneter Prädikation gesprochen werden, wie sie Rúžička als allgemeinstes Kennzeichen der untergeordnet prädikativen Partizipialstrukturen des Aksl. nennt, ${ }^{143}$ eben weil die Fokussierung Reflex des vom Sprecher intendierten Funktionstyps "Einheit einer Prädikation" ist. Hierin liegt die funktionale Affinität $z \mathrm{u}$ fokussierten sogenannten inhärenten Adverbialstrukturen, die auch als Attribute gegenüber dem Verbum ${ }^{144}$ bezeichnet werden und ihrerseits ja ebenfalls nicht den "synsemantischen" Charakter von $v$ zur Bedingung haben.

Ob man diese fokussierten Gerundial- (und Partizipial-)strukturen noch als untergeordnet prädikativ bezeichnet, ist eine Frage der Definitionsebene; eine untergeordnet prädikative Beziehung kann nur zwischen voneinander verschiedenen Größen etabliert werden, während hier auf der kommunikativ-semantischen Ebene eine Einheit intendiert ist analog zur Restriktivität innerhalb des Nominal-bzw. in unserem Rahmen Subjektskomplexes. ${ }^{145}$ Das hindert nicht, auf der syntaktischen Ebene und letztlich aufgrund angenommener logischer bzw. ontologischer Strukturen eine weitere Zerlegung in elementare Prädikationen vorzunehmen; dann aber besteht die Gefahr, den Unterschied zwischen kommunikativ obligaten und kommunikativ fakultativen Gerundien $z u$ verwischen, der relevant wird für die Distribution von Oberflächenkriterien und Paraphraseverhalten. U.E. ist es bei Strukturen des Typs on ušel ulybajas' am nahe-

$143 \mathrm{Vgl}$. Rúži ̌̌ka, SS, S. 160

$144 \mathrm{Vgl}$. Śmelev 76, S. 60ff, 65; vgl. die Paraphrase bei Comrie 74, S. 45: Eudesnoe penie Koli / Kolja poet čudesno: Adamec in Danes 74 (ed.), S. 194

$145 \mathrm{Vgl}$. Žaža 64, S. 182ff,u.ders. 65, S. 67ff zur "engen" Verknipfung von Part.-und Ger.strukturen mit dem Prädikat, konstituiert durch die aktuale Gliederung 
liegendsten, durchgehend Fokussierung und damit den als "Einheit einer Prädikation" umschriebenen Funktionstyp anzunehmen. Dem würde bei Rǔ̌i kka die Interpretation als interne periphere Prädikation ensprechen. ${ }^{146}$ RủZi $\chi_{k a}$ selbst allerdings, der nur das Kriterium der Reichweite der Negation des Prädikats, nicht aber die Koordinierung als Test anfürt, sieht hier den Typ "Advb" bzw. "VE", was im Sinne einer dreistufigen Gliederung des Prädikatskomplexes eher unserem Typ "eine Handlung mit ihrem Merkmal" entsprechen würde. Demnach wäre auch für das Russische die Nichtisolierung (ähnlich wie im Skr.) kein eindeutiges formales Kriterium für Fokussierung. Die Befragung von Muttersprachlern ergab kein einheitliches Bild; auch für Sätze nach dem Muster on ušel ulybajas' wurde Koordinierung als Paraphrase nicht ganz ausgeschlossen, die wir als Kennzeichen des Typs "eine Handlung mit ihrem Merkmal" angeführt hatten. Anani $\ell^{147}$ warnt vor"fehlerhaften" Konstruktionen des Typs*ureniki క̌li iz \$koly i smejas', die offenbar beim Unterricht russischer schüler auftreten und eventuell als Indiz für die Nichtfokussierung auch von ulybajas' im oben angeführten Beleg dienen können. Auch hier wärenzunächst einmal ausführliche Untersuchungen zur Satzintonation nötig. Aufgrund nur schriftlich vorliegenden Materials läßt sich die Frage nicht entscheiden. 148 Ein neuer strukturtyp tritt nicht auf, auch wenn man Ambiguität der nichtisolierten postponierten Gerundien nicht ausschließt.

Der Mangel an untergeordnet prädikativer selbständigkeit bei Fokussierung ist die strukturelle Voraussetzung für die Affinität zur Wortart Adverb (sowie bei restriktiven Partizipien für Ubergang in die Wortarten Adjektiv und Substantiv-s.o.). Daß die Unterscheidung zwischen kommunikativ obligaten und fakultativen Funktionstypen strukturelle Relevanz hat, zeigt auch die Verteilung von Endungstypen beim dialektalen Gebrauch von

146 Rúži ̌ka 66, S. 56ff zu Adv/PrädN; Weiss 77, S. 361ff; Rúそ̌i kka 65, Verbalphr., S. $352 \mathrm{ff}$

147 Ananic 72 , S. 64

148 Žǎ̌a 65, S. 185 vermutet andererseits für das Gerund in skazal, ulybnuv'is' phonetische Nichtisolierung 
russischen Partizipialkonstruktionen in dem von Kuz'mina / Nemčenko 71 angeführten Material. ${ }^{149}$

Die Zuordnung von möglichen Endungsformen zu Funktionstypen ergibt, daß hier sämtliche Endungen möglich sind in "prädikativer" und "prädikativ-attributiver" Verwendung, also bei Verwendung des Partizips innerhalb der prädikativen Basis und letztlich unabhängig von der von Kuz'mina/Nemcenko getroffenen Unterscheidung zwischen Null-Kopula, syn- und autosemantischen Verben. Nur ein Teil des Inventars an Endungen tritt auf bei Funktion außerhalb der prädikativen Basis, bei den Funktionstypen "prädikative Adverbialbestimmung" und "Adverbialbestimmung". Diese beiden sollen sich nach Kuz'mina/Nemčenko unterscheiden durch die Angabe eines Prozesses bzw. eines Zustandes; für die zuordnung von Endungen wird diese u.E. problematische Unterscheidung nicht relevant. Beide Male tritt die gleiche Teilmenge aus dem Gesamtinventar an Endungen auf.

Entscheidend für die Verteilung der Endungstypen ist also nicht die von Kuz'mina/Nemčenko in der Tabelle ubergeordnete Unterscheidung prädikativ/halbprädikativ/nichtprädikativ, begründet durch den charakter von $v$ und durch die lexikalisch-semantische Kennzeichnung des Gerund, sondern die sich hiermit uberschneidende Gliederung nach zugehörigkeit des Partizips zur prädikativen Basis/Stellung außerhalb der Basis.

Die Relevanz der Isolierung im Russischen ist strukturell wesentlich verankert durch das merkmallose Partizip in der asymetrischen Korrelation Partizip : Gerund. Die Isolierung dient zur Kennzeichnung der untergeordneten Prädikativität des potentiell ambigen Partizips. Es stellt sich die Frage, wie dann nichtisolierte Partizipien innerhalb des syntaktischen Prädikatskomplexes $z u$ bewerten sind, die üblicherweise als Prädikatsnomen, nichtisolierter prädikativer Determinant u.ä. bezeichnet werden.

Analog zu nichtisolierten Gerundien ist für die fokussierten Partizipien zunächst festzuhalten, daß sich das Problem ihrer

$149 \mathrm{Kuz}$ 'mina/Nemčenko 71, S. 8ff, 306ff 
funktionalen Spezifik auch bei sogenannten autosemantischen Verben stellt, d.h. bei Verben, die sehr wohl auch $z u$ selbständiger Prädikation ohne Erweiterung in der Lage sind. 150 Syntaktische Fakultativität ist nur eine notwendige, nicht aber hinreichende Bedingung zur Konstituierung des Funktionstyps.

Vgl. 2.B.:

Brat vernulsja domoj poveselevšij/poveselevśim

(Adamec II $75, \mathrm{~S} .32$ )

Igor' vozuraščaetsja zapychavšijsja (Svetlik 70,5.232)

Moǩt byt', ja vyidu iz plameni preobrazivšimsja, a?

(svetlik 70, S.232)

Mal'čik sidel pritichsij

(Adamec II 75, S.32)

On stojal na kladbišče postarev̌sij, peřal'nyj

(Křizková 69,PO,S.6)

Deti vozvraščalis' razrumjaniv̌siesja (Žaža 65,s.67)

Für alle ciese Belege gilt Unmöglichkeit der Paraphrase durch Koordinierung oder adverbialen Nebensatz infolge Fokussierung als Kriterium der kommunikativen obligatheit und unserer Umschreibung dieses Funktionstyps als "Einheit einer Prädikation". Als extreme Beispiele der Kondensierung vgl. mit (Null-)Kopula und Adjektivierung/Substantivierung: 151

On kurjaščij

(Petterson 72,S.21)

Ty nep'juščij

(Morison, S.63)

No èti nevygody byli tol'ko kažuščiesja

(Morison, S.61)

Die oben aufgeführten Belege beschreiben nicht eine Handlung in ihrem Verlauf, sondern nennen ein Ergebnis ${ }^{152}$ als Merkmal, anhand dessen das subjekt des Satzes identifiziert und in eine

150 Zur Variabilität der lexikalischen Semantik in Abhängigkeit von der aktualen Gliederung vgl. z.B. Buttke 69, S. 47ff, Pala in Danes 74 (ed.), S. 116ff, Adamec 66, P.s.. S.30ff, 42: vgl. auch Rư̌̌ička, SS, S. $168 f f$ zu potentiell halbkopulativen Verben

151 Vgl. a.a.O., s. 237

$152 \mathrm{Vgl}$. KY̌ižková 69, Synt.ch., S. $115 \mathrm{ff}$ 
durch dieses Merkmal definierte Klasse eingeordnet wird. ${ }^{153}$ Sie stehen in Gegensatz zur Nichtfokussierung nichtrestriktiver Strukturen und lassen sich u.E. am einfachsten nicht analog zu nichtrestriktiven strukturen ableiten von einer allgemeinsten Satzverknüpfung durch das "logische und", wie dies Weiss vorschlägt, sondern als restriktive strukturen zu einem zu tilgenden Nomen durch adnominale Einbettung. Dem entspricht die Affinität dieser fokussierten Strukturen zu Substantivierung/Adjektivierung.

Damit entginge man auch der widersprüchlichkeit im Ansatz von Křižková, wonach einerseits der prädikative Determinant ("PO") nur von nichtrestriktiven strukturen abzuleiten ist. andererseits aber die Funktion des fokussierten Po doch in Analogie zu Restriktivitat beschrieben wird. 154

Weiss 155 betont die Nichtfokussierbarkeit nichtrestriktiver Attribute und sieht im Rahmen prädikativ verwendeter NP's (im wesentlichen) nur restriktive Attribute vor. Bei FokusStatus der zu reduzierenden struktur verneint Weiss die "transformationelle verzweigungsmöglichkeit $z u$ eigentlich sekundärprädikativen Konstruktionen einerseits, nichtrestriktiven Attributen bzw. Attributsätzen andererseits." 156 Andererseits wird auch für strukturen, die üblicherweise als Prädikatsnomen beschrieben werden und die fokussiert sind. Darstellung anhand der Satzkonnexion durch das "logische und" vorgesehen. 157 Der Hinweis auf die analoge Nichtunterscheidbarkeit von "adverbnäheren" und "adverbferneren" Gerundialstrukturen 158 ist u.E. kein Argument, eben weil diese Nichtunterscheidbarkeit die syntaktische Ebene betrifft, die spezifik der fokussierten (Gerundial- und Partizipial)strukturen aber in jedem Fall nur auf der kommunikativ-semantischen Ebene $z u$ erfassen ist.

$153 \mathrm{Vgl}$. auch IsaCenko 63, Transf. an., S. 85

154 Vgl. Kłizková 69, PO, S. 8-9

155 Weiss 77 , S. 90ff, $356 \mathrm{ff}$

156 a.a.O., S. 367

157 a.a.o.. S. 362

158 a.a.o.. S. $275 f f, 362$ 
"Die Art der beteiligten Inhalte" sowie die "Länge der sekundärprädikativen Struktur" 159 sind allenfalls zusätzliche Kriterien, jedoch nicht struktureller Art.

Im Rahmen unseres Schematismus schlagen wir für fokussierte Strukturen folgende Darstellung vor:

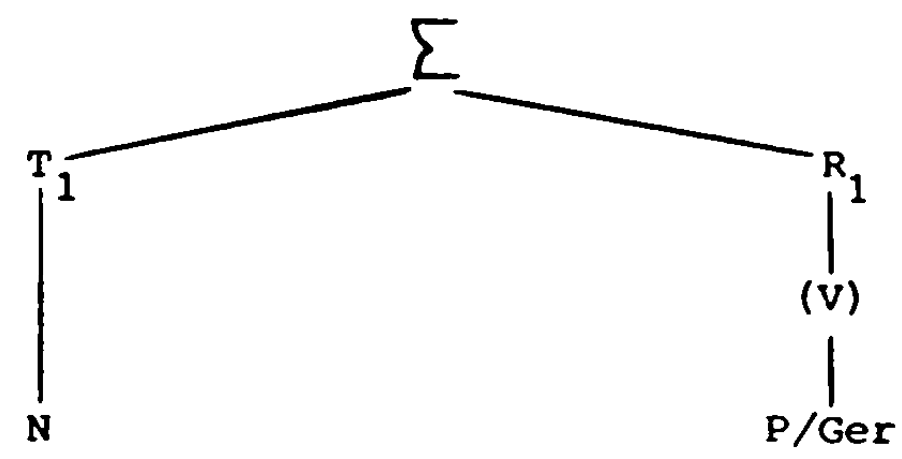

Das fokussierte Partizip/Gerund ist kommunikativ obligat, nur einschließlich des Partizip/Gerund ist die Prädikatshandlung als die vom sprecher intendierte zu identifizieren.

Dies gilt für Konstruktionen mit (Null-)Kopula, syn- und autosemantischem Verb; in jedem Fall liegt die Partizipial-bzw. Gerundialstruktur innerhalb der Reichweite der Negation des Prädikats und kann ihrerseits nicht paraphrasiert werden durch adverbialen Nebensatz oder Koordinierung. Auf der Ebene der kommunikativ-semantischen Gliederung liegt der Funktionstyp "Einheit einer Prädikation" vor, auch wenn syntaktisch und/oder logisch-semantisch eine weitere Zerlegung in elementare Prädikationen möglich ist, die dann aber Gefahr lauft, die spezifik des vorliegenden Funktionstyps zu neutralisieren. Syntaktisch evident wird dieser Funktionstyp in den Fällen, wo das Partizip/ Gerund alleine oder zusammen mit der Kopula auftritt und auch im Sinne der syntaktischen Darstellung nicht mehr Ableitung von einer zweibasigen struktur erfolgen kann.

159 a.a.o., s. 362 
9.

Zusammenfassung

Partizipial- und Gerundialkonstruktionen z.B. des Russischen sind als kondensierte strukturen exemplarisch für die asymmetrische Struktur des sprachlichen Zeichens.

Die Bestimmung von Funktionstypen der Partizipial- und Gerundialkonstruktionen anhand vorausgesetzter Satzglieder stößt auf Schwierigkeiten, nachdem diese satzglieder ungeklärte semantische Implikationen enthalten und in praxi auf einer Mischung formaler und nicht definierter semantischer Kriterien beruhen. Die funktionale Spezifik der Partizipial- und Gerundialstrukturen verweist uber die Grenzen des Syntagmas und der syntaktischen Ebene hinaus; als Formen der kondensierten Prädikation sind sie Formen des Ubergangs vom Satz zum Text; dieser ist nicht auf die Analogie zur syntaktischen struktur des Satzes anhand eines in der logischen Tradition stehenden paradigmatisch und referentiell begründeten Aquivalenzbegriffes $z u$ reduzieren.

Die Wahl der hochgradig fakultativen Partizipial- und Gerundialstrukturen statt potentiell äquivalenter sprachlicher formen und die Interpretation dieser hochgradig ambigen strukturen sind wesentlich bestimmt durch die kommunikative Intention des sprechers bzw. Hörers. Sie wird als konstitutiv angesehen für die Funktionstypen der Partizipial- und Gerundialkonstruktionen.

Aussichten auf eine zuordnung formaler und semantischer strukturen im Sinne der asymmetrischen Zeichenrelation ergeben sich nicht solange die Oberflächenkriterien Wortstellung, Isolierung und Realisierung der Korrelation eineindeutig vorausgesetzten Satzglied-| positionen mit ungeklärten semantischen Implikationen zugeordnet werden und/oder der Bereich des außersprachlich Bezeichneten zugrundegelegt wird bei der semantischen Interpretation.

Die Signifikanz der genannten oberflächenkriterien und der spezifisch sprachliche Begriff des Satzes und der untergeordneten Prädikativität verweisen auf die in der Kommunikation konstituierte sprachliche Bedeutung.

Im Aksl. besteht die Funktion der Langform des Partizips wesentlich in der Signalisierung der Nichtprädikativität, die Kurzform 
tritt attributiv-nichtprädikativ auf insbesondere in festen Wendungen und bei Restriktivität, d.h. bei kommunikativer obligatheit im Hinblick auf das ubergeordnete Nomen. Bei Nichtrestriktivität von $P_{k}$ gilt auf der Linie der Referenzidentität in den allermeisten Fällen periphere Prädikativität und Bezug auf das satzschließende Prädikat. In diesen Fällen sichert der Gebrauch der merkmalhaften Langform Attributivität.

Im Russischen sichert umgekehrt der Gebrauch des nach der Umwertung der Korrelation merkmalhaften Gerund (von einigen zweifelhaften Fällen abgesehen) Nichtattributivität bei Nichtrestriktivität und schliebt restriktive Interpretation eines grundsätzlich statt des Gerund möglichen Partizips aus. Während im Aksl. für die Langform des Partizips Nichtprädikativität gesichert ist, im Skr. nur noch das Gerund, d.h. das merkmallose Glied der aksl. Korrelation in gesicherter nichtattributiver Funktion auftritt und z.B. im Poln. akt. Partizip und Gerund nicht in Korrelation, sondern in Opposition stehen, d.h. nicht durch einander ersetzbar sein können. ist aufgrund der asymmetrischen Korrelation im Russischen das Kriterium der Isolierung strukturell verankert durch die Möglichkeit der untergeordnet prädikativen Funktion des akt.Partizips auch im Prädikatskomplex. Erweist sich z.B. im Skr. die Isolierung als fakultatives Grenzsignal und allenfalls die Nichtisolierung als obligat. so ist im Russischen die Isolierung obligat für untergeordnet prädikatives Partizip und Gerund bei Bezug auf das Satzganze. Das nichtattributive russ. Partizip tritt entweder nichtisoliert auf bei Fokussierung oder isoliert bei Satzbezug: nicht belegt ist kommunikativ fakultatives Partizip mit Bezug nur auf das Prädikat. Das Gerund ist obligat isoliert bei Satzbezug, jedoch ist die Isolierung nicht eindeutig, insofern als auch kommunikativ fakultatives isoliertes Gerund mit Bezug nur auf das Prädikat häufig ist. Nichtisolierung des Gerund ist in den allermeisten Fällen bedingt durch Fokussierung, doch kann auch Ambiguität des nichtisolierten Gerund (hinsichtlich Bezug nur auf das Prädikat bei kommunikativer Fakultativität) nicht ausgeschlossen werden. Im Skr. ist mit Nichtisolierung bei allen drei Funktions- 
typen zu rechnen.

Als aussichtslos erweist sich der versuch einer "Klassifizierung" nach gleichzeitig paradigmatisch und referentiell begründeten "Varianten" der vorausgesetzten Satzgliedposition "adverbiale Bestimmung". Hingegen eröffnet sich die Möglichkeit einer systematischen Bewertung der Oberflächenkriterien und einer Differenzierung nach strukturtypen analog zur tiefenstrukturellen Differenzierung der Adverbialbestimung, wenn als funktionstypkonstituierend die kommunikative Intention zugrundegelegt wird.

Dabei ergibt sich eine dreistufige Gliederung, die gegenüber traditionellen Umschreibungen von Funktionstypen U.E. den Vorteil hat, (nicht-eindeutig) beziehbar auf oberflächenkriterien, anhand von Tests empirisch überprüfbar und vergleichsweise konsistent zu sein.

Es wurde unterschieden einerseits zwischen komunikativ fakultativen Strukturen mit Bezug auf das Satzganze oder mit Bezug auf das Prädikat und andererseits kommunikativ obligaten Strukturen, für die analog zu restriktiven strukturen innerhalb von NP untergeordnet prädikative Selbständigkeit nicht mehr gegeben ist. Demgegenüber wird U.E. traditionellerweise nicht hinreichend differenziert zwischen Bezug auf das Satzganze und Bezug auf das Prädikat (so auch noch bei Kŕlžková und Rúźið̌ka) sowie zwischen kommunikativ fakultativen und obligaten strukturen (so noch bei Weiss 77).

Syntaktische Fügungseigenschaften und lexikalisch-semantische Charakteristik der beteiligten Komponenten reichen nicht aus zur Unterscheidung dieser Funktionstypen, d.h. insbesondere: alle drei Typen treten auf bei sogenannten autosemantischen Verben. So sehr wir der Kritik bei Weiss an einer syntaktischen Differenzierung beipflichten, U.E. ist die funktionstypkonstituierende Relevanz der sprecherintention insbesondere für kommunikativ obligate strukturen $z u$ betonen, die in einer $z u$ modifizierenden Tiefenstruktur ihren Platz finden müBte, um evidenten funktionalen Unterschieden Rechnung $z u$ tragen. Die Rekonstruierbarkeit eines identischen subjekts ist kein hinreichendes strukturkriterium. 
Innerhalb der syntaktischen Ebene scheint die Auseinandersetzung um das zusammengesetzte oder komplexe Prädikat, die im Hintergrund auch noch der von Weiss bezweifelten Differenzierung des Prädikatskomplexes bei Rúzicka nach Prädsec/Advb/ PrädN-Adv steht, nicht entscheidbar; in jedem Fall handelt es sich um mehrgliedrige strukturen, denen rekonstruierbare Konstituentensätze mit identischem subjekt zugrundeliegen. Darüberhinausgehende Unterscheidungen sind u.E. nicht syntaktisch $2 u$ begründen (vgl. unsere Kritik an Kacala), sondern anhand präzisierter strukturkriterien auf der Ebene der in der Kommunikation konstituierten sprachlichen Bedeutung. 
Abraham, W. / Binnick, R.J. (ed.): Generative Semantik. Frankfurt a. M. 1972

Adamec, P.: $K$ voprosu o sintaksiceskoj paradigmatike, in: Ceskoslovenská rusistika 11.1966. S. 76-80

- - Porjadok slov v sovr. russk. jaz.. in: Rozpr. Eslk. ak. vẻd., rada spol. věd. Rołnik 76, కeš. 15. Praha 1966 / P.s./

- - Očerk funkcional'no-transformacionnogo sintaksisa sovr. russk. jaz. I. ochobazovye predloženija. Praha 1973

- - Aktual'noe členenie, glubinnye struktury i perifrazy, in: Danes, F. (ed.): Papers on functional sentence perspective. Prague 1974. S. 189-195

- - Ołerk funkcional'no-trasformacionnogo sintaksisa sovr. russk. jaz. II. Dvuchbazovye predloženija s nefinitnymi k-strukturami. Praha 1975

Admoni, W. G.: Grundlagen der Grammatiktheorie. Heidelherg 1971 Allwood,J. / Anderson L.-G. / Dahl, Ö.: Logik für Linguisten. Tübingen 1973

AnaniZ, A. V.: Izučenie osložnennogo predloženija. Moskva 1972

Angelowa, G.: Die Partizipialkonstruktionen in den altbulgarischen Sprachdenkmälern. Sofija 1929 (Diss. München)

Apresjan, Ju. D.: Ideen und Methoden der modernen strukturellen Linguistik. München 1972

- - Theorie der Paraphrase. München 1974

Arens, H.: Sprachwissenschaft - Der Gang ihrer Entwicklung von der Antike bis zur Gegenwart. Bd. 1: Von der Antike bis zum Ausgang des 19. Jh.s. Bd. 2: Das 20. Jh. Frankfurt a. M. 1974

Arutjunova, N. D.: Predloženie i ego smysl'. Logiko-semantičeskie problemy. Moskva 1976

Babby, L. H.: The syntax of gerunds in Russian. Bloomington, Indiana 1975

Banaru, V. I.: Nekotorye voprosy funkcional'noj perspektivy predloženija (na materiale francuzkogo jazyka). Kišinev 1975

Barnet, V.: Vývoj systému participi aktivnich v ruštině. Praha 1965

Bartsch, R. / Vennemann, Th.: Semantic structures. Frankfurt a. M. 1972 
Bartsch, R.: Adverbialsemantik. Die Konstitution logisch-semantischer Repräsentationen von Adverbialkonstruktionen. Frankfurt a. M. 1972

- - The grammar of adverbials. A study in the semantics and syntax of adverbial constructions. Amsterdam, New York 1976

Belić, A.: A. Musić: značenie i upotreba participa u srpskohrvatskom jeziku. (Rez.), in: Južnoslovenski filolog 13. $1933 / 34$, S. 195-202

- - Istorija srpskohrvatskog jezika. Kn. II. sv. 2. Reči sa konjugaciom. Beograd 1969

Belošapkova, V. A.: Složnoe predloženie v sovr. russk. jaz. Moskva 1967

Birnbaum, H.: Untersuchungen zu den Zukunftsumschreibungen mit dem Infinitiv im Altkirchenslavischen, Ein Beitrag zur historischen Verbalsyntax des Slavischen. Stockholm 1958

- - Zur Aussonderung der syntaktischen Gräzismen im Altkirchenslavischen - einige methodische Bemerkungen, in: Scandoslavica 4. 1958. S.239-257

- - Studies on Predication in Russian. I. Santa Monica, Clf. 1964

- - Zur Sprache der Methodvita, in: Cyrillo-Methodiana. Köln, Graz 1964. S. 329-361

- - Predication and the Russian Infinitve, in: To Honor Roman Jakobson. Vol. I. The Hague 1967

- - On the Grammatical status of Short Form Adjektives and Some Related Problems in Modern Russian, in: California Slavic Studies. Vol. IV. S. 162-199. Berkely 1967

- - On Deep Structure and Loan Syntax in Slavic, in: Studies in Slavic Linguistics and Poetics in Honor of $B$. $O$. Unbegaun. New York 1968. S. 21-32

- - Deep structure and typological linguistics, in: Birnbaum, H.: Problems of typological and genetic linguistics viewed in a generativ framework. The Hague, Paris 1970. S. $9-70$

- - Zum infiniten Ausdruck der Prädikation bei Johannes dem Exarchen, in: Studia Palaeoslovenica Josepho Kurz septuagenario dedicatum. Prag 1971. S. 37-74

Bivon, R.: Element order. Studies in modern Russian language 7. Cambridge 1971

Björck, G.:- HN $\triangle I \triangle A \Sigma K \Omega N$-.Die periphrastischen Konstruktionen im Griechischen. Uppsala 1940

Bogdanović, D.: Poraba participa i glagolskih adveraba za prošlost u istorickim radnjama vuka Stef. Karadžića, in:

Nastavni vjesnik 8. 1900. S. 247-256 
Borkovskij, V. J.: Sintaksis drevnerusskich gramot. Moskva 1958

Borodič, V. V.: O kategorii opredelennosti / neopredelennosti $v$ staroslavjanskom jazyke, in: Slavjanskaja filologija, Vyp. 5. 1963. S. 162-202

Bragina, A. A.: O dvojstvennom charaktere sinonimii, in: Russkij jazyk v škole 3. 1978. S. 81-86

Brave, L. Ja.: K voprosu o znaČenii deepričastij soveršennogo vida, in: Russkij jazyk v łkole 6. 1940. S. 116-131

Bucharin, V. I.: Grammatika sovr. russk. lit. jazyka. M. "Nauka" 1970 (Zametki po kommunikativnomu sintaksisu) in: Russkij jazyk v škole 3. 1973. S. 118-122

Bungarten, Th.: Partizipialkonstruktionen in der deutschen Gegenwartssprache. Disseldorf 1976

Buravceva, A. D.: Stilistikeskaja rol' pričastnych oborotov i vozmožnost' zameny pridatoknych, in: Gor'kovskij prosveščenec $\mathrm{Nr}$. 7-8. 1934. S. 18-24

Buttke, K.: Gesetzmäßigkeiten der Wortfolge im Russischen. Halle/S. 1969

Comrie, B.: Transformationsanalyse russischer Nominalisierungen, in: Notizen und Materialien zur russischen Linguistik Unterlagen für die Seminararbeit. Frankfurt a.M. 1974. S. 38-46

Conrad, R.: Transformationsanalyse russischer Infinitivkonstruktionen - Eine Beschreibung der Wortverbindungen mit abhängigem Infinitiv auf der Basis des appl. gen.Modells. Halle/s. 1969

Coseriu, E.: Einführung in die transformationelle Grammatik Autorisierte Nachschrift, besorgt von $G$. Narr und $R$. Windisch. Tübingen 1970

- - Leistung und Grenzen der transformationellen Gramatik. Tübingen 1975

Ceremisina, N. V.: Invariantnye modeli dinamičeskich struktur russk. pov. predloženija, in: Voprosy sint. russk. jaz. Rostov-na Donu 1971. S. 26-34

Cernachovskaja, L. A.: Perevod i smyslovaja struktura. Moskva 1976

Česnokova, . L. D. : Konstrukcii s predikativnym opredelitelem, in: Russkij jazyk 1. 1972. S. 80-82

- - O fakul'tativnosti i objazatel'nosti komponentov sintaksiceskoj struktury, in: Voprosy sint. sovr. russk. 1it. jaz. Rostov-na Donu 1973. S. 3-10

Dahl, ర.: Topic and Comment: a study in Russian and general transformational grammar. Göteborg 1969 
- - Topic and comment, contextual boundness and focus. Hamburg 1974

- - Topic-comment structure revisited, in: Dahl, ठ.. (ed.): Topic and comment, contextual boundness and focus. Hamburg 1974. S. 1-24

- - Topic-comment structure in generative grammar with a semantic base, in: Danes, F. (ed.): Papers on functional sentence perspective. Prague 1974. S. 75-80

Daneš, F. (ed.): Papers on functional sentence perspective. Prague 1974

- - Functional sentence perspective and the organization of the text, in: Danes. F. (ed.): Papers on functional sentence perspective. Prague 1974. S. 106-128

- - u.a.: Zur Terminologie der funktionalen Satzperspektive. in: Danes., F. (ed.): Papers on functional sentence perspective. Prague 1974. S. 217-222

- - u.a.: Projekt einer theoretischen Konzeption der tschechischen Grammatik, in: Zeitschr. für slawistik 20. 1975. S. 613-630

Deribas, L. A.: Vido-vremennye značenija deepričastij v sovremennom russkom jazyke, in: Russkij jazyk v śkole 5. 1954. S. $1-7$

Dezsö, L. / Szépe, G.: Two problems of topic-comment, in: Daneš, F. (ed.): Papers on functional sentence perspective. Prague 1974. S. 81-86

- - Contribution to the topic-comment problem, in: Dahl. ö. (ed.): Topic and comment, contextual boundness and focus. Hamburg 1974. S. 65-93

Dozorec, 2. A.: Nekotorye voprosy obosoblenija, in: Russkij jazyk v škole 1972, 6. S. 96-101

Dressler, W.: Einführung in die Textlinguistik. Tübingen 1972

- - Funktionelle Satzperspektive und Texttheorie, in: Daneš. F. (ed.): Papers on functional sentence perspective. Prague 1974. S. 87-105

Dvořák, E.: Vývoj prechodnikových konstrukci ve starši češtině. Praha 1970

Ebeling, C. L.: Subject and Predicate-Especially in Russian, in: Dutch Contributions to the 4 th International Congress of Slavistics. S.-Gravenhage 1958. S. 1-38

Ehrich, V. / Fincke, P. (ed.): Beiträge zur Grammatik und Pragmatik. Kronberg/Ts. 1975

Fedorov, A. K.: Trudnye voprosy sintaksisa. Moskva 1972

Ferrell, J.: A Note on the History of the Form of the Russian Gerund in $-a$, in: Wiener Slavistische Jahrbiicher 12 . 1965. S. 13-17 
Filipović, N.: Die Partizipialkonstruktionen in der deutschen dichterischen Prosa von heute. Tübingen 1977

Fillmore, Ch. J.: The Position of Embedding Transformations in a Grammar, in: Word. Vol. 19.1963. S. 208-231

Firbas, J.: Some aspects of the Czechoslovak approach to problems of functional sentence perspective, in: Danes. F. (ed.): Papers on functional sentence perspective. Prague 1974. S. 11-37

Flier, M. S.: V. A. Belołapkova: SloŽnoe predloženie v sovr. russk. jazyke (Rez.), in: International Journal of slav. Linguistics and Poetics 13. 1970. S. 194-198

Forssmann, Th.: Die Ubertragung der griechischen Participialconstructionen in dem Ostromir'schen Evangelium. Straßbuing 1877

Frančuk, E. N.: Upotreblenie obosoblennych priłastnych oborotov $i$ opredelitel'nych pridatoknych predloženij v sovr. russk. jaz. (Avtoref.) Moskva 1952

Frantz, D. G.: Generative semantics - an introduction, with bibliography. Bloomington. Indiana 1974

Frei, H.: Charactérisation, indication, spécification, in: For Roman Jakobson. Essays on the Occasion of his sixtieth birthday. The Hague 1956. S. 161-168

Friederich, w.: Probleme der Semantik und Syntax des englischen Gerundiums. München 1973

Gabka, K.(red.): Syntax. (Bd. 3 in: Gabka, K. (red.): Die russische Sprache der Gegenwart). Leipzig 1976

Gajsina, R. M.: Sredstva rečevogo kontakta $v$ otnošenii $k$ strukture predloženija, in: Voprosy sintaksisa. Rostov-na Donu 1971. S. 36-44

Galkina-Fedoruk, E. M.: Suždenie i predloženie. Moskva 1056

Gallis, A.: The Syntax of Relative in Serbocroatian. Viewed on a Historical Basis. Oslo 1956

Gardiner, S. C.: Russian Participles, in: Studies in Slavic Linguistics and Poetics in Honor of B. O. Unbegaun. New York 1968. S. 47-54

Gazisova, R. F.: O soßetaemosti kačestvennych prilagatel'nych $v$ pozici skazuemogo (na mat. russk, povestej XV-XVII vV.) in: Voprosy sint. russk. Jaz. Rostov-na Donu 1971. S. 72 $-80$

Georgieva, V. L.: Istorija sintaksičeskich javlenij russk. jaz. Moskva 1968

Gil'čnok, T. E.: Vlijanie vnutrennogo konteksta na skazuemoe (na materiale predloženij s nesoglasovannym skazuemym), in: Voprosy sint. sovr. russk. lit. jaz. Rostov-na Donu 1973. S. 23-31 
Gnevko, V. T. u.a.: Sovremennyj russkij jazyk. Minsk 1975

Grabe, V.: Obščee značenie sintaksičeskoj konstrukcii i transformacii, in: Ceskoslovenská Rusistika XI. 1966.

S. $72-75$

Grannis, 0. C.: Some semantic aspects of relative clause in English, in: IRAL vl. XIII/2, 1975. S. 111-118

Grepl, M.: Die semantisch modifizierenden Komponenten der Satzstrukturen, in: Zeitschr. für Slawistik 20. 1975. S. 631-637

Grochowski, M.: Grundlagen semantischer Verknüpfungen zwischen prädikativen Ausdrücken, in: Zeitschr. für Slawistik 20. 1975. S. 673-681

Grünenthal, O.: Die Ubersetzungstechnik der altslavischen Evangelienübersetzung, in: Arch. f. slav. Phil. Bd. 31. 1910. S. 321-366. Bd. 32. 1911. S. 1-48

Gvozdev, A. N.: Sovremennyj russkij lit. jazyk. T. 1.2. Moskva 1973

Halliday, M. A.: The place of "functional sentence perspective" in the system of linguistic description, in: Danes, F. (ed.): Papers on functional sentence perspective. Prague 1974. S. 43-53

Hartung, W.: Die zusammengesetzten Sätze. Berlin 1964. ${ }^{6} 1973$

Heidolph, K. E.: Kontextbeziehungen zwischen Sätzen in einer generativen Grammatik, in: Kybernetika 2. 1966. S. 274281

Heine, R.: Vermutungen zum lateinischen Partizip, in: Gymnasium 79. 1972. S. 209-238

Hjelmslev, L.: Le verbe et la phrase nominale, in: Mélanges de philologie. Offerts à J. Marouzeau. Paris 1948

Herrielin, E.: Uber den Gebrauch der Präsenspartizipien von perfektiven Verben im Altkirchenslavischen. (Diss.) Uppsala 1935

Höck, Ch.: Untersuchungen zur Syntax altserbischer Partizipialkonstruktionen. Magisterarbeit (unveröff. Typoskript). München 1974 / M. /

Holk, A. G. F. van: Definite and indefinite in old church slavonic. A contribution to the theory of the linguistic sign, in: Dutch Contributions to the 5 th International Congress of Slavistics. The Hague 1963. S. 93-123

Immler, M.: Generative Syntax - Generative Semantik. Darstellung u. Kritik. München 1974

Isað̌enko, A. V.: Transformacionnyj analiz kratkich i polnych prilagatel'nych, in: Issledovanija po strukturnoj tipologii. Moskva 1963. S. 61-93 
- - Porjadok slov v poroždajuščj modeli jazyka, in: Českosl. přednásky pro VI. mezinárodni sjezd slavistù $v$ Praze. Praha 1968. S. 51-59

- - Die russische \$prache der Gegenqart. I. Formenlehre. München 1968. 1975

Issledovanija po sintaksisu staroslavjanskogo jazyka. Contributions to Old Church Slavonic Syntax. Prag 1963

Ivanovna, V. F.: Nekotorye voprosy sintaksi keskoj sinonimiki opredelitel'nych pridatoxnych predloženij i pricastnych oborotov, in: Russkij jazyk v łkole. 2. 1958. S. 73-78

Ivić, M.: Theoretische und methodologische Aspekte des Begriffs der Kondensation, in: Zeitschr. für Slawistik 20. 1975. S. 774-780

Jacobsson, Gö.: The Use of Gerunds and Active Participles in Modern Russian Newspapers. Göteborg 1969

Jacobsson, Gu.: The Slavic Active Participles: Original Structure and Interference, in: Scandoslavica 9. 1963. S. 123 $-138$

Jäger, G.: Zur Klassifizierung komplexer Sätze im Tschechischen und Polnischen. Halle/S. 1968

Jakobson, R.: Zur Struktur des russischen Verbums, in: Charisteria Guilelmo Mathesio. Prag 1932. S. 74-84

- - Russian Conjugation, in: Word IV. 1948. S. 155-167

- - Shifters, Verbal Categories and the Russian Verb. Harvard University Press 1957

- - Linguistische Aspekte der Ubersetzung, in: Form und Sinn Sprachwissenschaftliche Betrachtungen. München 1974.

S. 154-161

Jelitte, H.: Studien zum Adverbium und zur adverbialen Bestimmung im Altkirchenslavischen. Eine morphologisch-syntaktische Untersuchung. Meisenheim a. G. 1961

Jurčenko, V. S.: Tipy skazuemogo, in: Russkij jazyk v škole 4. 1973. S. 79-83

Kačala, J.: Das sekundäre Prädikat, in: Zeitschr. für slawistik 14. 1969. S. 700-711

- - Doplnok v slovenčine. Bratislava 1971

- - Grammatische und semantische Aspekte der syntaktischen Kondensation, in: Zeitschr. für Slawistik 20. 1975. S. 797-801

Kade, 0.: Transformationelle Analyse russischer Gerundialkonstruk. tionen zur Ermittlung von Ubersetzungsäquivalenzen, in: zeitschr. für slawistik 13.1968. S. 601-615

Kalakuckaja, L. P.: Ad-ektivacija pricastij v sovr. russk. lit. jaz. Moskva 1971 
Kanšin, J. A.: Pričastnye $i$ deepričastnye konstrukcii v roli členov predloženija ( $K$ voprosu o vyraženii と̌lenov predloženija nedelimymi sočetanjami slov), in: Russkij jazyk

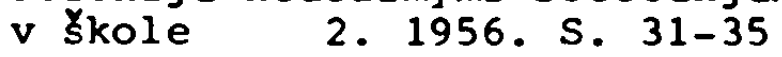

Karcevskij, S.: Système du verbe russe. Genf 1927

Koljadenko, G. S.: Iz nabljudenij nad determinirujuščimi obstojatel'stvami $v$ strukture složnogo sintaksičeskogo celogo. in: Russkij jazyk v skole 3. 1972. S. 86-89

Kolšanskij, G. V.: Grammati ̌eskaja funkcija obosoblenija ‘́lenov predloženija, in: Naư̌nye doklady vysšej školy.god izd. 5. 1962. S. 31-41

Kononenko, V. I.: Sinonimika sintaksičeskich konstrukcij v sovr. russk. jaz. Kiev 1970

Kornilov, V. A.: Postpositivnyj pričastnyj oborot kak komponent atributivno-predikativnogo sočetanija slov, in: Voprosy izučenija russkogo jazyka. Rostov-na Donu 1964. S. 95-99

- - Predloženija s pričastnymi oborotami v sovremennom russkom literaturnom jazyke. (Avtoref.) Moskva 1967

- - Dva tipa priðastnych oborotov, otnosjaščichsja k podležaščemu, in: Voprosy sint. russk, jaz. Rostov-na Donu 1971. S. 92-101

Koschmieder, E.: Der Einfluß des Aspekts auf den Formenbestand des polnischen Verbums, in: Arch. f. slav. Phil. 41. 1927. S. 262-295

- - Die noetischen Grundlagen der Syntax. Abhandlungen der Bayer. Akademie d. Wissenschaften 4. 1951. München 1952

- - Das Allgemeingültige in der Syntax, in: Welt der slawen 4. 1959. S. 369-389

- - Beiträge zur allgemeinen Syntax. Heidelberg 1965

- - Der Prädikator, in: Münchener Studien zur Sprachwiss. H. 26. München 1969. S. 63-76

Kovaliv, P.: Participial adjectives in the slavic languages. Winnipeg 1957

Kovtunova, I. I.: o sintaksičeskoj sinonimike, in: Voprosy kul'tury reči I. Moskva 1955. S. 115-142

- - Aktual'noe členenie i sistema jazyka (na materiale russk. jaz.), in: Danes, F. (ed.): Papers on functional sentence perspective. Prague 1974. S. 142-151

Krenn, H.: Die grammatische Transformation. Minchen 1974

Křižková, H.: Problemy prostogo predloženija, in: Československá rusistika XII. 1967. S.76-81

/ Problemy /

- - $K$ voprosu o tak nazyvaemoj appozicii (na materiale russkogo jazyka), in: Travaux linguistiques du Prague. 1968. S. 33-45 
- - Predikativnoe opredelenie i struktura predloženija $v$ sovremennom russkom jazyke, in: Slavia 38. 1969. S. $1-35$ / po /

- - Syntaktická charakteristika pŕechodnikových konstrukci. in: Bulletin ústavu ruského jazyka a literatury XIII. 1969

/ Synt. ch. /

- - Immenoe skazuemoe i struktura predloženija $v$ sovremennych slavjanskich jazykach, in: International Journal of slavistic Linguistics and Poetics XIII. 1970. S. 15-49

- - Adverbiálni determinace píitinná, in: studie $z$ výcholoslovanské jazykovědy (Sbornik věnovaný vII. mezinárodnímu sjezdu slavistú ve Varకavě). Praha 1973. S. 182-222

Krjučkov, S. E. / Maksimov, L. Ju.: Sovremennyj russkij jazyk sintaksis složnogo predloženija. Moskva 1969

Krylova, O. A.: Ponjatie mnogojarusnosti aktual'nogo členenija i nckotorye sintaksið̌eskie kategorii, in: Naučnye dokl. vysšej skkoly, filolog. nauki 5. 1970. S. 86-91

Krylova-Samojlenko, o. A.: o predikativnosti, in: Naučnye dokl. vyš̌ej skoly, filolog. nauki 1. 1965. S. 135-139

Kubik, M.: $K$ transformacionnoj interpretaçii sloźnopoď̌inennych predloźenij v russkom jazyke, in: Čskoslovenská rusistika XI. 1966. S. 80-86

- - K voprosu klassifikacii prostogo predloženijja $v$ novoj akademičeskoj grammatike russk. jaz.. in: Ceskoslovenská rusistika XVII. 1972. S. 65-69

- - (red.): Sintaksis russk. jazyka. Praha 1974 / sint. r.j./

Kürzinger, J.: Die Heilige Schrift des Alten und Neuen Testamentes nach den Grundtexten ubersetzt und herausgegeben von Prof. Dr. V. Hamp, Prof. Dr. M. Stenzel, Prof. Dr. J. Kiurzinger. Aschaffenburg 1956

Kuz'mina, I. B. / Nemčenko, E. V.: Sintaksis pričastnych form $v$ russkich govorach. Moskva 1971

Kuznecov, P. S.: K voprosu o skazuemostnom upotreblenii prićastij i deepričastij v russkich govorach, in: Materialy i issledovanija po russkoj dialektologii. T. 3. Moskva 1949. S. 59-83

Lakoff, G.: Instrumental adverbs and the concept of deep structure, in: Foundations of language 4/1. 1968. S. 4-29

Lapteva, O. A.: Nerešennye voprosy teorii aktual'nogo členenija, in: Voprosy jazykoznanija 2. 1972. S. 35-47

Lees, R. B.: The Grammar of Englisch Nominalisations, in: International Journal of American Linguistics. Vol. 26. 1960 
Lekant, P. A.: Tipy i formy skazuemogo v sovr. russk. jaz. Moskva 1966

- - Razvitie form skazuemogo, in: Mysli o sovr. russk. jaz. Moskva 1969. S. 140-154

Lépissier, J. : Les emplois du gérondif en vieux slave, in: Studia Palaeoslovenica. Praha 1 71. S. 215-220

Lerot, J.: Zur Integration der Semantik in die Transformationsgrammatik, in: Beiträge zur Linguistik und InformationsVerarbeitung 18.1970, S. 7-21

Leskien, A.: Grammatik der serbo-kroatischen Sprache. 1. Teil. Lautlehre, Stammbildung, Formenlehre. Heidelberg 1914 Heidelberg 1976

Leška, o.: Mesto transformacionnych otnos̀enij v jazykovoj strukture, in: Československá rusistika 11. 1966. S. 65-66

Lomtev, T. P.: očerki po istoričeskomu sintaksisu russkogo jazyka. Moskva 1956

- - Predloženie i ego grammatiČskie kategori1. Moskva 1972

Malaščenko, V. P.: O semantiČeskoj svjazi svobodno prisoedinjaemych predložnych konstrukcij s ostal'noj Cast'ju predloženija, in: Voprosy sintaksisa russk. jaz. Rostov-na Donu Donu 1971. S. 113-120

- - O priznakach determinirujušc̀ich členov predloženija, in: Voprosy sintaksisa sovr. russk. Iit. jaz. Rostov-na Donu 1973. S. 11-21

Mann, T.: Doktor Faustus. Žizn' nemeckogo kompozitora Adriana Leverkjuna, rasskazannaja ego drugom. Perevod s nemeckogo S. Apta i Natalii Man. Moskva 1975

McCawley, J. D.: Wo kommen Nominalphrasen her?, in: Kiefer, F. / Perlmutter, D. M. (ed.) : Syntax und Generative Grammatik 2. Frankfurt a. M. 1974

Mel'ničuk, A. S.: Razvitie predikativnogo upotreblenija pričastij na $-(v),-(v)$ ś $v$ vostočno-slavjanskich jazykach, in: Slov'jans'ke movoznavstvo, zbirnik statej I. Kyiv 1958

Meyer, P. G.: Satzverknüpfungsrelationen. Ein Interpretationsmodell für situationsunabhängige Texte. Tübingen 1975

Michnevič, A. E.: Sintaksi Ceskaja kondenzacija i tipy polipredikativnych struktur, in: Zeitschr. für slawistik 20. 1975. S. 788-796

Miklosich, F. (ed.): Monumenta serbica spectantia historiam Serbiae Bosnae Ragusii. Wien 1858. (Nachdruck: Graz 1964) / MS /

- - Vergleichende Grammatik der slavischen Sprachen. Bd. 4. Vergleichende Syntax der slavischen Sprachen. Wien 1883

Miko, F.: The generative structure of the slovak sentence Adverbials. The Hague, Paris 1972 
Milejkovskaja, G. M.: K istorii dejstvitel'nych pri Castij nastojałcego vremeni $v$ russkom jazyke, in: Russkij jazyk v tkole. Bd. 19.1958. S. 12-19

Morison, W. A.: The present gerund and active participle. London 1959

Mrázek, R.: Problema skazuemogo i ego klassifikacii (na materiale russkogo i Ceskogo jaz.), in: Sbornik praci filosofické fakulty Brněnské university. Bd. 7. 1958. S. 10-34

- - Sintaksiłeskie otnoł̌nija i členy predloženija, in: Sbornik praci filosofické fakulty Brnénské university. Bd. 10. 1961. S. 47-60

Mučnik, I. P.: Grammatičeskie kategorii glagola $i$ imeni v sovr. russk. lit. jaz. Moskva 1971

Mulisch, H. (red.): Morphologie (Bd.2 in:Gabka,K. (red.):Die russische Sprache der Gegenwart) Düsseldorf 1975

Musić, A.: Relativne rečenice u hrvatskom jeziku, in: Rad jug. ak. znan. i um. Bd. 138. Zagreb 1899

- - ZnaXenje i upotreba participa u srpsko-hrvatskom jeziku, in: Rad jug. ak. znan. i um. Bd. 250. Zagreb 1935

Nemec, I.: O otázce staroslovenských participii praes. act. sloves dokonavých, in: Slavia 26.1957. S.1-12

Nikolaeva, T. A.: Aktual'noe Elenenie - kategorija grammatiki teksta, in: Voprosy jazykoznanija 2. 1972. S. 48-54

Novák, P.: Remarks on devices of functional sentence perspective, in: Danes, F. (ed.): Papers on functional sentence perspective. Prague 1974. S. 175-178

Paffen, K. A.: Die Hauptregeln der russischen Grammatik. 2. Teil: Satzlehre. 3.. bearb. Aufl. Halle/S. 1969

/ Paffen II /

- - Die Hauptregeln der russischen Grammatik. 3. Teil: Stillehre. Halle/S. 1960

/ Paffen III/

Pala, K.: Semantic classes of verbs and FSP, in: Daneš, F. (ed.): Papers on functional sentence perspective. Prague 1974.

S. 196-207

Panfilov, V. Z.: Grammatika i logika. Grammatičeskoe členenie prostogo predloženija. Moskva, Leningrad 1963

- - Grammar and logic. The Hague, Paris 1968

- - Vzaimootnošenie jazyka i myšlenija. Moskva 1971

- - Wechselbeziehungen zwischen Sprache und Denken. Berlin 1974

Panzer, B.: Strukturen des Russischen. München 1975

Pešikan, M.: Neke napomene orazvoju aktivnih participa, in: Zbornik za filologiju i lingvistiku 2. Novi Sad 1959. S.88-106 
Pettersson, Th.: On Russian Predicates. Göteborg 1972

Petöfi, J. S.: Transformationsgrammatiken und eine ko-textuelle Texttheorie. Grundfragen und Konzeptionen. Frankfurt a. M. 1971

Pfister, R.: Schlußwort $z u$ den Thesen $z u$ Linguistik und Sprachunterricht, in: Gymnasium 79.1972. S. 314-330

Pičugov, Ju. S.: Osobennosti izučenija temy "predloženija s obosoblennymi દlenami" po novomu uČebniku, in: Russkij jazyk v škole 6. 1973. S. 31-39

Pisarkova, K.: Möglichkeiten und Erfordernisse der linguistischen Textanalyse - Ansatz zur Interpretation von drei Textfragmenten aus dem Werk von Thomas Mann (am Beispiel der poln. (tbersetzung), in: Zeitschr. für Slawistik 20. 1975. S. 698-703

Popović, I.: Istorija srpskohrvatskog jezika. Novi Sad 1955

Porunkevič, D. A.: Ob izučenii deepriČastija v VI. klasse, in: Russkij jazyk v škole 3. 1972. S.72-74

Potebnja, A. A.: Iz zapisok po russkoj grammatike. Bd. 1.2. Moskva 1958

Prager Autorengruppe: Einfuhrung in die generative Semantik. Kronberg/Ts. 1975

Protčenko, J. F.: O substantivirovannych prilagatel'nych i pričastijach so znaČeniem lica, in: Russkij jazyk v škole 4. 1958. S. 7-11

Pumjanskij, A. L.: Informacionnaja rol' porjadka slov $v$ naučnoj i techničeskoj literature. Moskva 1974

Raspopov, I. P.: Stroenie prostogo predloženija v sovr. russk. jaz. Moskva 1970

Rath, R.: Die Partizipialgruppe in der deutschen Gegenwartssprache. Duisseldorf 1971

Regula, M.: Wesen, Arten und Formen der Apposition und des Attributs, in: Ze1tschr. fur franz. Sprache und Lit. Bd. 78. 1968. S. $102-138$

Ressel, G.: Studien zur generativ-transformationellen SemantoSyntax russ. Adverbialkonstruktionen. Minchen 1974

Resetar, M.: Elementargrammatik der kroat. (serb.) Sprache. Zagreb 1922

Ries, J.: Was ist ein Satz? Beiträge zur Grundlegung der Syntax. H. III. Prag 1931

Rohrer, Ch.: Funktionelle Sprachwissenschaft und transformationelle Grammatik - Die Verwandlung von Satzteilen im Französischen. Minchen 1971

Rudnev, A. G.: O proischoždenii deepričastija, in: Časopis pro slovanské jazyky, literatury a dĕjiny SSSR 1. 1956. S19-63 
- - Sintaksis osložnennogo predloženija. Moskva 1959

- - Sintaksis sovr. russk. jaz. Moskva 1963. ${ }^{2} 1968$

Ruzić, R. H.: The Aspects of the Verb in Serbo-Croatian. University of California publications in mod. philology. Vol. 25, No. 2. Berkeley, Los Angeles 1943

Rưžička, R.: Griechische Lehnsyntax im Altslavischen, in: Zeitschr. für Slawistik 3. 1958. S. 173-185

- - Struktur und Dialektik in der russischen Grammatik, in: Zeitschr. für Slawistik 4. 1959

- - Struktur und Echtheit des altslavischen dativus absolutus, in: Zeitschr. für Slawistik 6. 1961. S. 588-596

- - Korrelationswandel als Erklärungsmodell in der diachronischen Grammatik, in: Zeitschr. für Slawistik 7.1962.

S. 678-685

/ Korr. /

- - Zur Rolle der asymmetrischen Korrelation in der historischen Syntax, in: Otázy slovanské syntaxe. Sbornik Brnénské syntaktické konference. Praha 1962. S. 186-189 / Zur Rolle /

- - Zur syntaktischen Typologie moderner slawischer Literatursprachen, in: Zeitschr. für Slawistik 8. 1963. S. 833 $-860$

/ Typ. /

- - Das syntaktische System der altslawischen Partizipien und sein Verhältnis zum Griechischen. (Habibschrift) Berlin 1963

/ ss/

- - Zur Syntax der Verbalphrase in der modernen russischen Literatursprache, in: Zeitschr. für Slawistik 10. 1965 S. 341-353

/ Verbalphr. /

- - Zur Spezifik der slawischen Sprachen bei der Strukturanalyse, in: Slavia 34. 1965. S. 407-411

- - - skizzen zu einer generativen Transformationssyntax der modernen russischen Literatursprache, in: International Journal of Slavic Linguistics and Poetics 9. 1965.

S. $62-81$

- - Studien zur Theorie der russischen Syntax, in: Sitzungsberichte der dt. Ak. d. Wiss. zu Berlin. Jg. 1966. Berlin 1966. S. 1-96

- - Korrelation und Transformation, in: To Honor Roman Jakobson. Vol. 3. The Hague 1967. S. 1709-1733

- - Betrachtungen zur Lehnsyntax im Altslawischen in: Studia Palaeoslovenica. J. Kurz sept. ded. Praha 1971. S.303-308

- - / walther, G.: Beziehungen zwischen Bedeutung und Syntax, in: Zeitschr.für Slawistik 19. 1974. S. 460-474 
- - O sootnošenii sintaksisa i znałenija predloženija,

in: Zeitschr. für Slawistik 20. 1975. S. 746-751

Schaller, H. W.: Die Wortstellung im Russischen. Slavistische Beiträge 21. München 1966

- - Die Bestimmung syntaktischer Eigenschaften bei nominalen Satzgliedern im Russischen, in: Scandoslavica 17. 1971. S. 161-169

Schmaus, A.: Lehrbuch der serbokroatischen Sprache. München, Belgrad 1969

Schmidt, F.: Logik der Syntax. Berlin 1957, 31961

- - Symbolische Syntax. München 1970

Schrenk, J.: Einfache und gefügte Sätze slavischer Schriftsprachen - Analyse und Darstellung. München 1968

Schwarz, Ch.: Pseudologik und Natürliche Generative Grammatik Anmerkungen zur "Adverbialsemantik" von Renate Bartsch, in: Papiere zur Linguistik 11. 1976. S. 56-109

Schwyzer, E.: Zur Apposition, in: Abhandlungen der dt. Ak. d. Wiss. zu Berlin. Jg. 1945/46. Berlin 1947

Seiler, H.: Relativsatz, Attribut und Apposition. Wiebaden 1960

Sgall, P.: Porjadok slov i aktual'noe členenie predložeñija v generativnom opisanii slavjanskich jazykov, in: Českoslov. předn. pro VI mezinar. sjezd slav. v Praze. Praha 1968. S. 61-65

- - Focus and contextual boundness, in: Dahl, ö. (ed.): Topic and comment, contextual boundness and focus. Hamburg 1974. S. 25-51

- - Zur Stellung der Thema-Rhema-Gliederung in der Sprachbeschreibung, in: Daneš, $F$. (ed.): Papers on functional sentence perspective. Prague 1974. S. 54-73

- - Zur Semantik der Deprädikation, in: Zeitschr. für Slawistik 20. 1975. S. 782-787

- - / Hajicová, E. / Benešová, E.: Topic, focus and generative semantics. Kronberg/Ts. 1973

Sirotina, O. B.: Porjadok slov v russk. jaz. Saratov 1965

Słonski, S.: Die Ubertragung der griechischen Nebensatzkonstruktionen in den altbulgarischen Sprachdenkmälern. Kirchhain N. -L. 1908

Stankova, M.: La traduction des gérondifs polonais en bulgare et en francais, in: Cahiers de linguistique d'orientalisme et de slavistique - Etudes slaves 3-4.1974. S. 187-206

Starikova, E. N.: Implicitnaja predikativnost' v sovr. anglijskom jazyke. Kiev 1974

Steblin-Kamenskij, M. J.: O predikativnosti, in: Vestnik Leningr. Univ. 20. Vyp. 4. 1956. S. 129-137 
Steinitz, R. / Lang. E.: Adverbialsyntax. Berlin 1969

Stefanović, M.: Popridevljivanje glagolskog priloga na -ći. in: Juznosl. filolog 18. 1949/50. S. 55-85

Stojanović, Lj.: Značenje glagolskih participa, in: Jǔ̌nosl. filolog 8. 1928/29. S. 1-12

Stola, R.: Zum Gebrauch der unbestimmten Formen von aktiven Partizipien in der Funktion von zweitrangigen Prädikaten im Lavrent'evskij spisok, in: Wiener slav. Jahrbücher. Bd. 5. 1956. S. 14-27

- - Zur Frage der Entwicklung des deepričastie im Altrussischen, in: Slavjanskaja filologija 1. 1958. S.189-194

suchotin, V. P.: Sintaksičeskaja sinonimika v sovr. russk. lit. jaz. (glagol'nye slovosocetanija). Moskva 1960

Svane, G. O.: Die Flexionen in Stokavischen Texten aus dem Zeitraum 1350-1400. (Sprachgeschichtliche Untersuchungen über älteres Serbokroatisch). Aarhus 1958

svetlik, J.: Sintaksis russk. jazyka $v$ sopostavlenii so slovackim (Syntax rustiny $v$ porovnani so slovenskou). Bratislava 1970

Sviblova, T. A.: $K$ voprosu ob otgraničenii obosoblennogo opredelenija ot schodnych grammatičeskich konstrukcij, in: $\mathrm{Na}-$ uknye doklady vyssej skoly, filolog. nauki. 1 (17) . 1962 S. $174-186$

Svoboda, K.: Podnètná práce o doplňku $v$ slovĕnštinè, in: Naše Rez. r. 58,2. 1975. S. 89-94 (Rezension zu Kacala 71)

Syčeva, S. N.: Upražnenija po sintaksičeskoj sinonimike, in: Russkij jazyk v Skole 3. 1973. S. 55-57

Śčeboleva, I. I.: Strukturnye tipy vstanych konstrukcij $v$ sovr. russk. jaz.. in: Voprosy sint. russk. jaz. Rostov-na Donu 1971. S. 101-113

Skarić, D. M.: Tvorba i poraba participa (gerundija) prezenta aktiva $i$ preterita aktiva 1 u Cakavskoj književnosti 15 i 16 vijeka, in: Nastavni vjesnik 10. 1902. S. 1-32

Śmelev, D. N.: Sintaksičeskaja členimost' vyskazyvanija $v$ sovr. russk. jaz. Moskva 1976

Śvedova, N. Ju. (red.): Grammatika sovr. russk. lit. jazyka. Moskva 1970

/ AG 70 /

- - O sootnošenii grammatiCeskoj i semantičeskoj struktury predloženija, in: Slavjanskoe jazykoznanie. VI meždunarodnyj s-ezd slavistov. Varłava, avg. 1973 g. Moskva 1973 S. 458-483

- - (red.) : Grammatičeskoe opisanie slavjanskich jazykov. Kon cepcii i metody. Moskva 1974 
Tauscher. E. / Kirschbaum, E.-G.: Grammatik der russischen Sprache. Düsseldorf 1968

Té̌ak, S. / Babić, S.: Pregled gramatike hrvatskosrpskog jezika. Zagreb 1969

Tjukšina, L. A.: Sintaksičeskie funkcii deepričastnych konstrukcij v sovremennom russkom jazyke. (Diss.) Moskva 1951

Tolstoj, N. I.: Značenie kratkich i polnych form prilagatel'nych $v$ starslavjanskom jazyke (na materiale evangelskich kodeksov), in: Voprosy slavjanskogo jazykoznanija, vyp. 2. 1957. S. 43-122

Uličný, O.: K syntagmatické a transformačni charakteristice doplǔku, in: Slovo a slovesnost 30. 1969. S. 11-22

- - Ještè k pojeti doplňku, in: slovo a slovesnost 31. 1970. S. $271-278$

Uhlírová, L.: On the role of statistics in the investigation of FSP, in: Danes, F. (ed.): Papers on functional sentence perspective. Prague 1974. S. 208-216

Ungeheuer, G.: Paraphrase und syntaktische Tiefenstruktur. IPKForschungsberichte 4. Bonn 1968

Uspenskij, B. A.: Opyt transformacionnogo issledovanija sintaksičeskoj tipologii, in: Issledovanija po strukturnoj tipologii. Moskva 1963. S. 52-60

Vaillant, A.: La langue de Dominko zlatarić, poète ragusain de la fin du XVI siècle. Bd. 1.2. Paris 1928/1931

- - La formation de la langue littéraire serbo-croate, in: Revue des études slaves. T. 28. 1951. S. 80-92

- - Grammaire comparée des langues slaves. T. 2. Morphologie. Lyon 1958

- - Grammaire comparée des langues slaves. T. 3. Paris 1966

Valgina, N. S.: O dvustoronnej sintaksiceskoj svjazi $v$ sovr. russk. jazyke, in: Russkij jazyk v škole 5. 1972.

S. 99-104

Valimova, G. V.: K voprosu o kommunikativnych edinicach, in: Voprosy sint. russk. jaz. Rostov-na Donu 1971. S. 21-26

Večerka, R.: Ke konkurenci vztażných vět a participii v staroslovènstine, in: Sbornik praci filosofjcké fakulty Brnènské university. Bd. 10. 1961. S. 35-45/Ke konkurenci/

- - Syntax aktivných participii v staroslověnštině. Praha 1961

Vinogradov, V. V.: Russkij jazyk - grammatičeskoe učenie o slove. Moskva, Leningrad 1947

Vitek, S. J.: Functions of Russian adverbs. A preliminary transform analysis. Ann Arbor, Michigan 1967 
Vlasov, A. K.: Deepriłastnyj oborot ne otnesennyj k podležaš̉emu, in: Russkij jazyk v škole 19.1958. S. 35-38

Voigt, $W$ (red.): Leitfaden der russischen Grammatik. München 1971

/ Leitfaden /

Vondrák. W.: Vergleichende slavische Grammatik. Bd. II. Formenlehre und Syntax. 2. Auflage neu bearb. von 0 . Grünenthal. Göttingen 1928

Weber, H.: Das erweiterte Adjektiv und Partizipialattribut im Deutschen. Müchen 1971

Weiss, D.: Zur Modellierung poln. Gerundialkonstruktionen, in: Slavistische Linguistik - Studien und Texte Nr. 6/8.

Referate des 1. Konstanzer slavistischen Arbeitstreffens (25.9.-27.9.1974) Bremen 1975

/ Weiss 74 /

- - Syntax und Semantik polnischer Partizipialkonstruktionen im Rahmen einer generativ-transformationellen Sprachbeschreibung. Bern, Frankfurt a. M., Las Vegas 1977

Zimek, R.: Semantičeskij aspekt sintaksičeskoj transformacii, in: Československá rusistika 9.1966. S. 66-72

Zimmermann, I.: Der Parallelismus verbaler und substantivischer Konstruktionen in der russischen Sprache der Gegenwart. in: Zeitschr. für Slawistik 12. 1967. S.744-755

zolotova, G. A.: očerk funkcional'nogo sintaksisa russkogo jazyka. Moskva 1973

Zaza, S.: $K$ voprosu obosoblennych členov predloženija, in: Sbornik praci fil. fakulty Brněnské university. roč. 13. 1964. S. $181-187$

- - K voprosu o sintaksičesko-semantičeskom i aktual'nom c̀lenenii sloźnogo predlożenija, in: Sbornik praci fil. fakulty Brněnské university. roč. 14.1965. S. 67-73

- - K problematice větosledu a aktuálniho Kleněni podradného souveti $v$ rustine, in: Ceskosl. płednášky pro vi. mezinárodn. sjezd slav.v Praze. Praha 1968. S. 175-179

N A C H T R A G

Biricz, E.: Serbokroatisch. Bd. II. (Ubungen für den Sprachunterricht). Wien o. J.

Birnbaum, H.: Linguistische Beobachtungen an einem altrussischen Text, in: IJSLP 3. 1960. S. 45-68

- - Obsceslavjanskoe nasledie $i$ inojazycnye obrazcy $v$ strukturnych raznovidnostjach staroslavjanskogo predlozenija, in: American contributions to the sixth internat. congress of slavists, Praque 1968.Vol.I. The Haque/Paris 1968. S.29-63 
Bukatevic, N. I. u.a. (ed.): ocerki po sravnitel'noj grammatike vostocnoslav. jazykov. Odessa 1958

Hamm, J.: Grammatik der serbokroatischen Sprache. Wiesbaden 1967

Jelitte, H.: Zur Entwicklung der Satzstrukturanalyse im Russischen, in: Slavistische Studien zum VII. Internat. Slavistenkongre $B$ in Warschau 1973. Herausg. von $J$. Holthusen u.a. München 1973. S. 189-207

Kłizková, H.: Temporal'no-kvantitativnaja determinacija glagola (opyt transformacionnogo analiza), in: Ceskoslovenská rusistika 11. 1966. S. 86-93

Loseva, L. M.: K izuCeniju mezfrazovoj svjazi (Abzac i sloznoe sintaksiceskoe celoe), in: Russkij jazyk v skole 1. 1967. S. 89-94

Michnevic, A. E.: Nekotorye voprosy izucenija sintaksiceskich (podxinitel'nych) svjazej slov $v$ rabotach sovetskich i Cechoslovackich lingvistov, in: Voprosy jazykoznanija 5. 1968. S. 106-112

Mistrik, J.: Ausdrucksmittel der Hypersyntax, in: Zeitschr. für Slawistik 20. 1975. S. 643-646

Ressel, G.: Syntaktische Struktur und semantische Eigenschaften russischer Sätze. Generativ-semantische und modelltheoretische Untersuchungen zu einer Paraphrasengrammatik des Russischen. München 1977

Rúzicka, R.: Integration slawischer und nichtslawischer Sprachen an der syntaktischen Peripherie. Ein besonderer Typ von Gerundialstrukturen, in: Zeitschr. fiir Slawistik 18. 1973. S. 453-479

Sergeeva, Z. A.: Tipy svjazoðnych glagolov $v$ substantivnych predlozenijach, in: Voprosy sintaksisa sovr. russk. lit. jaz. Rostov-na-Donu 1973. S. 32-40

Zimmermann, I.: Zur Problematik der Kondensation, in: Zeitschr. für Slawistik 20.1075. S. 804-807 